\title{
Production of Extremely High-Lying States in Strong Microwave Fields
}

Alexandr Arakelyan

Almaty, Kazakhstan

B.S., Novosibirsk State University, 2009

A Dissertation presented to the Graduate Faculty of the University of Virginia in Candidacy for the Degree of

Doctor of Philosophy

Department of Physics

University of Virginia

May, 2015 
(C) Copyright by Alexandr Arakelyan All Rights Reserved

May 2015 


\section{Abstract}

This dissertation investigates phenomena related to interactions of Rydberg atoms with strong microwave fields in the presence of small static fields on the order of $5-10 \mathrm{mV} / \mathrm{cm}$. New aspects of microwave ionization of alkali Rydberg atoms at 17 and 38-GHz fields are presented, and the results of a novel experiment conducted at $80 \mathrm{GHz}$ are discussed. Most important, the production of extremely high-lying states is observed at all microwave frequencies. About $\sim 5-15 \%$ of the excited atoms are found in such states after the end of strong many-cycle microwave pulse in two regimes. When the laser excitation of Rydberg population occurs during the microwave pulse, the observed spectra show a regular structure of peaks spaced by a microwave photon from the ionization limit for a wide range of binding energies, both above the continuum and far below it. When the excitation happens in zeromicrowave field, atoms are also found in the extremely high-lying states. In both cases, the high-lying states are observed for low Rydberg states, in the regime where the spacing between Rydberg states is much larger than the microwave frequency. Our observations at $80 \mathrm{GHz}$ suggest that this phenomenon occurs due to a multiphoton excitation of atoms to the limit and is not a result of microwave ionization of atoms, but rather a distinct process that occurs along with it. 


\section{Acknowledgments}

This work would not have been completed without people that surrounded me during the past six years. My labmates, J. Nunkaew, H. Park, and D. Norum, were of great help when I first joined the group and was learning to survive in the laboratory environment. E. Magnuson, V. Carrat, J. Lee, S. Niyaz, and K. Wijayaratne, who joined the lab after me, helped a lot by instigating stimulating discussions during the group meetings. Especially, I would like to thank Eric and Vincent for their support not only during working hours but also after it. I should not forget to mention my fellow graduate students with whom I became friends while taking classes the very first year at UVA and who accompanied me during my years in Charlottesville.

The Physics Department at UVA is lucky to have a wonderful staff. R. Marshall, C. Floyd, L. St. John, D. Wilmer, V. Ingram, B. Martyn, P. Joseph, and B. Guyton were always ready to help with countless troubles I encountered in a very fast and effective manner. Special thanks to H. McLaughlin, D. Shifflett, and T. Shifflett who had to solve my problems for me, sometimes on a daily basis. Without all these people, the research work that is conducted at this department would have been completely impossible. I am very grateful to Professors L. Bloomfield and R. Jones who were kind to serve on my advisory committee. Their advice, scientific guidance, and discussion of my research problems helped me to keep on track and successfully finish my work at UVA.

It is impossible to express how grateful I am and how much I owe to my advisor, Professor Thomas F. Gallagher. He is a great scientist and the best advisor a student can dream of. His immeasurable patience, kindness, and support made my experience in the graduate school very enjoyable and effective. 
Special thanks go to my wife Natalya who is always my greatest supporter. Without her I would not have been able to get where I am now. 


\section{Contents}

1 Introduction 1

1.1 Rydberg Atoms . . . . . . . . . . . . . . . . . . 2

1.1.1 Atomic units ...................... 3

1.1 .2 Quantum Defects . . . . . . . . . . . . . . . . . . 3

1.1.3 Static Field Ionization . . . . . . . . . . . . . . . . . . . 4

Hydrogenic ionization . . . . . . . . . . . . . . 4

Nonhydrogenic Ionization . . . . . . . . . . . . . 7

1.1.4 Tunneling and Multiphoton Ionization . . . . . . . . . . . . 8

1.1.5 Scaled Frequency . . . . . . . . . . . . . . . . . . . . . 9

1.2 Background . . . . . . . . . . . . . . . . . . . . 10

1.2.1 Microwave Ionization . . . . . . . . . . . . . . . . . . . 10

1.2 .2 High-lying states . . . . . . . . . . . . . . . . . . 16

2 Experimental Apparatus $\quad 20$

2.1 Vacuum Apparatus . . . . . . . . . . . . . . . . . . 20

2.1.1 Two-stage Pumping System . . . . . . . . . . . . . . . . . 21

2.1 .2 Thermal Beam . . . . . . . . . . . . . . . . . 22

2.1.3 Chamber Maintenance . . . . . . . . . . . . . . . . . . 23

2.2 Optical Excitation System . . . . . . . . . . . . . . . . . . . . . . . . 24

2.2.1 $\mathrm{kHz}$ Pump Laser System . . . . . . . . . . . . . . . . . . . 24

2.2 .2 Atomic Energy Levels . . . . . . . . . . . . . . . . . 26

2.2 .3 Dye Lasers . . . . . . . . . . . . . . . . . . . . . . . . . . . 27

2.3 Microwave System . . . . . . . . . . . . . . . . . . . . . . 30

2.3.1 Microwave Components . . . . . . . . . . . . . . . . . 31

2.3.2 Microwave Cavities . . . . . . . . . . . . . . . . . . 32

2.4 Detection and Data Acquisition Methods . . . . . . . . . . . . . 34

2.4.1 Field Ionization Pulse . . . . . . . . . . . . . . . . . . 34

2.4.2 Microchannel Plate Detector . . . . . . . . . . . . . . . . . 37

2.4 .3 Data Acquisition . . . . . . . . . . . . . . . . . . 38

2.5 Canceling Static Stray Fields _ . . . . . . . . . . . . . . . . . 39

2.6 Rydberg Spectrum . . . . . . . . . . . . . . . . . . . . . . . 39

3 Ionization of Rydberg atoms by 17,38 , and $80-\mathrm{GHz}$ microwave fields 43

3.1 Introduction . . . . . . . . . . . . . . . 43

3.2 Ionization of $\mathrm{Li}$ and $\mathrm{Na}$ Rydberg atoms by $17-\mathrm{GHz}$ microwave fields . . . 44

$3.2 .1 \quad$ Experimental Approach . . . . . . . . . . . . . . . 45 
3.2 .2 Experimental Observations . . . . . . . . . . . . . . 46

Lithium . . . . . . . . . . . . . . . . 46

Sodium . . . . . . . . . . . . . . . . . 48

3.3 Ionization of Li Rydberg atoms by $38-\mathrm{GHz}$ microwave fields . . . . . . . . 53

3.3.1 Experimental Approach . . . . . . . . . . . . . . 53

3.3.2 Experimental Observations . . . . . . . . . . . . . 54

3.4 Ionization of $\mathrm{Na}$ Rydberg atoms at $80 \mathrm{GHz} \ldots \ldots \ldots \ldots$

3.4.1 Experimental Approach . . . . . . . . . . . . . . . 61

3.4 .2 Experimental Observations . . . . . . . . . . . . . 62

3.5 Conclusions . . . . . . . . . . . . . . . . . . 77

4 Spectrum of quasi-stable states in strong microwave fields $\quad \mathbf{7 9}$

4.1 Introduction . . . . . . . . . . . . . . . . . . 79

4.2 Spectrum of quasi-stable states of $\mathrm{Li}$ in $38-\mathrm{GHz}$ field . . . . . . . . . 80

4.2 .1 Experimental Approach . . . . . . . . . . . . . . . 80

4.2 .2 Experimental Observations . . . . . . . . . . . . . . 81

4.3 A spectrum of the high-lying states of $\mathrm{Na}$ in $80-\mathrm{GHz}$ field $\ldots \ldots$. . . . . 90

4.3.1 Experimental Approach . . . . . . . . . . . . . . . . . . 91

4.3.2 Experimental Observations . . . . . . . . . . . . . . . . . . . 92

4.4 Conclusions . . . . . . . . . . . . . . . . . . . . 100

5 Production of extremely high-lying states by strong microwave fields 103

5.1 Introduction . . . . . . . . . . . . . . . . . . 103

5.2 Production of extremely high-lying states by 38-GHz microwave field in $\mathrm{Li} 105$

5.2 .1 Experimental Approach . . . . . . . . . . . . . . . . . 105

5.2 .2 Experimental Observations . . . . . . . . . . . . . . . . 105

5.3 Production of extremely high-lying states of Li and $\mathrm{Na}$ by 17-GHz fields . . 112

5.3.1 Experimental Approach . . . . . . . . . . . . . . . . 112

5.3.2 Experimental Observations . . . . . . . . . . . . . . . 113

5.4 Production of extremely high-lying states in 80-GHz fields . . . . . . . . . . 120

5.4 .1 Experimental Approach . . . . . . . . . . . . . . . . 121

5.4 .2 Experimental Observations . . . . . . . . . . . . . . . . . 121

5.5 Conclusions . . . . . . . . . . . . . . . . . . . 127

6 Conclusions $\quad 131$

$\begin{array}{ll}\text { Bibliography } & 134\end{array}$ 


\section{List of Figures}

1.1 Coulomb and modified Coulomb-Stark potential of Eq. 1.5. . . . . . . . . .

1.2 Calculated energy level map as a function of static field amplitude for the $n=19,20,21|m|=0$ states of $\mathrm{H}(\mathrm{a})$ and $\mathrm{Na}(\mathrm{b})$. The central state is $n=20$.

1.3 Field-ionization signal and microwave-ionization signal for the $\mathrm{Na} 20 \mathrm{~s}$ state showing the ionization threshold as a disappearance of the field-ionization signal or the appearance of the microwave-ionization signal with increasing microwave power, from $[13] \ldots \ldots \ldots \ldots$

1.4 Simplified Stark-level diagram for sodium. The bold arrows indicate the first steps leading ultimately to ionization at the classical ionization limit. Updated from [14]. . . . . . . . . . . . . . . . . . .

1.5 Observed spectra in varying strengths of microwave field overlaid on an energy-level diagram of the $\mathrm{Na} m=0$ states, from [19]. The spacing between the zero-field $n=25$ and $26 d$ states is $397 \mathrm{GHz}$, and, at the highest field shown, the spacing is filled with a structure of 26 peaks spaced by the microwave frequency. . . . . . . . . . . . . . .

1.6 Field ionization signal as a function of excitation energy for different microwave fields. The energy scale is relative to the $B a^{+} 6 p_{1 / 2}$ limit. The dotted line and dashed line show the maximum recombination energy predicted by Eqs. 1.18 and 1.19, respectively. Updated from [38]. . . . . . . . . . . .

2.1 Optical diagram of the pump beam. The output pulse of the 527-nm pump beam is 'sliced' by two Pockels cells (PC) resulting in two 20-ns pump beams. The first pulse travels through a half-wave plate (HW), a 50/50 beam splitter and pumps two dye lasers (DL) that drive the first two atomic transitions. Passing through a 1-m converging lens, the second 20-ns pulse pumps another dye laser that drives atoms to a Rydberg state. For convenience, the polarization of laser pulses is shown ( $\mathrm{H}$ for horizontal, $\mathrm{V}$ for vertical). To populate $3 p-3 d$ transition of $\mathrm{Na}$, a cw Toptica diode laser is used. Its output goes through two optical isolators (IS) and is 'sliced' in a Pockels cell to form a step function in such a way that this laser is on immediately after the first dye laser pulse. All output laser beams driving atomic transitions are polarized vertically, unless extra half-wave plates are introduced to rotate the laser polarizations. . . . . . . . . . . . . . . . . 
2.2 Timing of the 527-nm pulses after passing through Pockels cells. The first pulse pumps two first dye lasers, the second one pumps the last transition dye laser, while the long tail is sent to a dump. The picture is updated from $[47] \ldots \ldots \ldots \ldots \ldots \ldots \ldots$

2.3 Energy level diagram to excite Li $n p$ and Na $n f$ Rydberg states. . . . . . .

2.4 Schematic diagram of a Littman-Metcalf type dye laser provided by S. Niyaz. A cylindrical lens focuses the pump beam inside the dye cell, and a fluorescent dye flowing trough it serves as an active medium for the laser cavity that consists of two mirrors. The diffraction grating ensures relatively narrow linewidth of the laser output. . . . . . . . . . . . . . . .

2.5 A photograph of an IR Littman-Metcalf type dye laser that I used to drive the $2 p-3 s$ transition in Li. The tuning mirror is mounted to a precision rotating stage to ensure easy and fast tuning of the laser frequency. . . . . .

2.6 Two brass mirrors shown constitute an $80-\mathrm{GHz}$ microwave cavity. Laser beams cross each other and the atomic beam at the center of the cavity. The mirrors are $69.7 \mathrm{~mm}$ in diameter with $52-\mathrm{mm}$ radii of curvature. . . . . . .

2.7 Laser beams cross each other and the atomic beam to form an interaction region at an antinode of the microwave electric field at the center of the 80$\mathrm{GHz}$ cavity. The FIP is applied to the bottom plate, and charged particles are pushed towards the MCP detector through a hole in the plate above the interaction region. The microwave field is fed through a small iris in one of the mirrors with a WR-12 waveguide connected to it. . . . . . . . . . .

2.8 A circuit diagram to generate a FIP provided by K. Wijayaratne. Two sets of values are shown. A circuit with the top set components generates a regular FIP with a $3-\mathrm{kV}$ output at $100-\mathrm{V}$ input at $1 \mathrm{kHz}$ repetition rate. The other set includes high-power resistor and capacitor to generate $8-\mathrm{kV}$ FIP at $300-\mathrm{V}$ input at the same repetition rate. This circuit is very large as it includes two big AC fans for cooling purposes. . . . . . . . . . . . . .

2.9 An oscilloscope trace showing the FIP, taken with the help of a high-voltage probe, and the trigger pulse. The FIP has $1.3-\mathrm{kV}$ amplitude and $1.16-\mu s$ rise time at $30-\mathrm{V}$ input. . . . . . . . . . . . . . . . 37

2.10 The MCP circuit and connection diagram. . . . . . . . . . . . . 38

2.11 The interaction region is surrounded by metal plates and cavity mirrors from all sides, each of which can be separately biased to control the static field distribution. The $80-\mathrm{GHz}$ cavity is shown that had to be mounted at a 45 degree angle to the atomic beam propagation. Laser and atomic beams reach the interaction region through the holes drilled in the side plates. In case of the $80-\mathrm{GHz}$ cavity, the spacing between brass mirrors is about $10 \mathrm{~cm}$, side plates $-8 \mathrm{~cm}$, bottom ant top plates $-10 \mathrm{~cm}$. . . . . . . . . . . . 
2.12 Field ionization signal as a function of the laser frequency tuning, in terms of energy relative to the ionization limit (IL). The horizontal axis is calibrated by the etalon and optogolvanic signals. The first peak on the graph corresponds to $n=56$, at $-1056 \mathrm{GHz}$. Atoms are excited in the presence of a $18.5-\mathrm{mV} / \mathrm{cm}$ static field that depresses the IL by $25 \mathrm{GHz}$. Signal above the IL ( 0 on the $\mathrm{x}$-axis) is zero, as the electrons created in laser photoionization leave the interaction region before the onset of the FIP and are not detected. In this case a survival probability is measured. . . . . . . . . . . . .

3.1 Experimental timing diagram. Rydberg states created in laser excitation are subjected to the microwave pulse. About $300 \mathrm{~ns}$ later a slowly rising FIP ionizes atoms, and either electrons or ions are detected by the MCP, depending on the polarity of the FIP. . . . . . . . . . . . .

3.2 Microwave ionization of the $\operatorname{Li} 73 p$ (a) and $58 p$ (b) state. Fraction of atoms surviving the MW field as a function of its strength detecting electrons from all bound states of $n \geq 55$ (circles) and fraction of atoms apparently ionized by the MW field as a function of its strength observed by detecting ions with the positive FIP (squares). The apparent microwave ionization signal collected with the positive FIP is due to both microwave ionization and field ionization of atoms in state of $n \geq 90$. Because the high-lying states are present in both signals, they are not complementary. . . . . . . . . . .

3.3 Microwave ionization of the $\mathrm{Na} 73 f$ state. Fraction of atoms surviving the MW field as a function of its strength detecting electrons from all bound states of $n \geq 55$ (circles) and fraction of atoms apparently ionized by the MW field as a function of its strength observed by detecting ions with the positive FIP (squares). Data is taken in the presence of $10-\mathrm{mV} / \mathrm{cm}$ (a), and $35-\mathrm{mV} / \mathrm{cm}$ (b) static field. The apparent microwave ionization signal collected with the positive FIP is due to both microwave ionization and field ionization of atoms in state of $n \geq 90$. Because the high-lying states are present in both signals, they are not complementary an (a). However, when the static field is added, both detection methods yield the same result. . .

3.4 Fractional apparent ionization for the Na $24 f$ (filled circle), $30 f$ (filled triangle), and $40 f$ (filled square) states obtained with a positive FIP. Vertical lines correspond to the $50 \%$ ionization threshold of $|\mathrm{m}|=0$ and 1 states measured by Pillet et al. [13]. The $40 f$ state clearly exhibits two thresholds, while lower $30 f$ and $24 f$ states have one threshold, but do not reach the second one, as not the $100 \%$ of atoms are ionized. . . . . . . . . . .

$3.5 \mathrm{Na} 40 f(\mathrm{a}), 30 f(\mathrm{~b})$, and $24 f$ (c) signal obtained with the positive FIP vs microwave field for static field $E_{s}$ values from $10 \mathrm{mV} / \mathrm{cm}$ to $2.5 \mathrm{~V} / \mathrm{cm}$. The vertical dotted lines correspond to the $50 \%$ ionization thresholds as measured by Pillet et al. [13] at $0.28 n^{5}$ for $n=40,30$, and 24 . For $30 f$, with $E_{s}=10$ $\mathrm{mV} / \mathrm{cm}$ the $|m|=0$ threshold is roughly $68 \mathrm{~V} / \mathrm{cm}$, and the $|m|=1$ threshold is at $E=1 / 9 n^{4}=700 \mathrm{~V} / \mathrm{cm}$, too high a field to be observed. As the static field is raised, the $|m|=1$ threshold moves to join the $|m|=0$ threshold at $E=1 / 3 n^{5} \ldots \ldots \ldots \ldots \ldots \ldots$ 
3.6 The static field dependence of the high-lying state signal and the microwave ionization probability of the $30 f$ after exposure to a $120-\mathrm{V} / \mathrm{cm}$ microwave pulse. The high-lying state signal observed by detecting electrons with a negative FIP (solid line). The microwave ionization probability observed by detecting ions with a positive FIP (dashed line). The half widths of the two curves differ by a factor of $40 \ldots \ldots \ldots \ldots \ldots$

3.7 Experimental timing diagram for the investigation of the microwave ionization of $\mathrm{Li}$ by a $38.34-\mathrm{GHz}$ microwave field. . . . . . . . . . . . . .

3.8 Field ionization signal as a function of the laser frequency tuning, in terms of energy relative to the ionization limit (IL), after exposure to a microwave pulse of amplitude: (a) $0 \mathrm{~V} / \mathrm{cm}$, (b) $36 \mathrm{~V} / \mathrm{cm}$ and (c) $90 \mathrm{~V} / \mathrm{cm}$. The horizontal axis is calibrated by the etalon and optogolvanic signals. The first peak on the graphs corresponds to $n=37$, at $-2410 \mathrm{GHz}$. . . . . . . . . .

3.9 (a) MW fields as a function of atomic energy for $50 \%$ and $10 \%$ ionization. Vertical dotted lines on graphs correspond to the energies of Rydberg states for which the classical Kepler frequency equals $3 \omega, 2.5 \omega, 2 \omega, 1.5 \omega, \omega$, and $0.5 \omega$ respectively, where $\omega=38.34 \mathrm{GHz}$ is the MW frequency. Our observations for the $10 \%$ field are in good agreement with theoretical calculations made by Krug and Buchleitner for the ionization of Li by a 36-GHz MW field (red dotted line) for $1 / 3<\Omega<1$. To our knowledge, no calculation exists for $10 \%$ ionization fields at $38.3 \mathrm{GHz}$ in the region from $-500 \mathrm{GHz}$ to the IL. (b) An expanded view of the $50 \%$ ionization fields in the $\Omega>1$ regime. Our result is in excellent agreement with a prediction of Jensen et al. [25] (red dashed line) that the average ionization field does not depend on $n$. That prediction, however, is not intended to reproduce the experimental structure that we observe. As shown by the vertical dotted lines spaced by $\omega$, the structure in the ionization fields is at the microwave frequency. . . . . . . .

3.10 (a) Rydberg state distribution as a function of energy with the microwave field off. The distribution shown are gray scale representations of oscilloscope traces of time resolved field ionization signals. Black is a large signal and white is no signal. (b) The FIP is shown on the same vertical time scale. Rydberg states ionize at two different regimes: adiabaticaly at $1 / 16 n^{4}$ and diabaticaly at $1 / 9 n^{4} \ldots \ldots \ldots \ldots \ldots$

3.11 Final state distributions as a function of energy for: (a) $36-\mathrm{V} / \mathrm{cm}$, (b) $90-$ $\mathrm{V} / \mathrm{cm}$ MW fields. The distributions shown are gray scale representations of oscilloscope traces of time resolved field ionization signals. Black is a large signal and white is no singal. For convenience the vertical scale is shown both as time and the field of the FIP. The dashed lines in (a) and (b) show the diabatic ionization field $\left(1 / 9 n^{4}\right)$, while the dotted lines correspond to the adiabatic ionization field $\left(1 / 16 n^{4}\right)$. Both 36 and $90-\mathrm{V} / \mathrm{cm}$ plots show the presence of the extremely high-lying states that are ionized within the first 50 ns of the FIP. The redistribution to states other than the initial or extremely high-lying occurs to both higher and lower binding energies, but in no case do we observe a continuous distribution from the initial state to the IL. In (b) an additional peak set between 70 and $100 \mathrm{~ns}$ is due to electrical ringing and is an artifact of the experiment. . . . . . . . . . . . . . 
3.12 Experimental timing diagram. (a) Atoms are excited in the presence of the 79.05-GHz microwave (MW) field. (b) Rydberg states created in laser excitation are subjected to the microwave pulse. About $300 \mathrm{~ns}$ after the microwave pulse, a slowly rising FIP ionizes atoms, and either electrons or ions are detected by the MCP, depending on the polarity of the FIP. . . . . . . . . .

3.13 A total excitation spectrum of atoms excited in the presence of the $85-\mathrm{V} / \mathrm{cm}$ $80-\mathrm{GHz}$ microwave field. Ions are detected. Zero-field Rydberg states are partially ionized at this field, and sidebands emerge around them. The first large peak on the left corresponds to $n=30$. The FIP amplitude is about $800 \mathrm{~V} / \mathrm{cm}$, and it is capable of ionizing $n>25(-5260 \mathrm{GHz})$ states. . . . .

3.14 Field ionization signal as a function of the laser frequency, in terms of energy relative to the ionization limit (IL). (a) A zero-field spectrum of Rydberg states in the range of $26 \leq n \leq 40$. We detect only $f$ states due to vanishingly small oscillator strength of the transition to an $n p$ state. (b) A total excitation spectrum of atoms excited in the presence of $170-\mathrm{V} / \mathrm{cm} 80-\mathrm{GHz}$ microwave field. Ions are detected. The spectrum exhibits extra peaks between Rydberg states due to emerging sidebands of zero-field states. Sidebands are an integral number of microwave photons from the corresponding zero-field Rydberg state. Sidebands of different peaks overlap producing an irregular structure of peaks. . . . . . . . . . . . . . . .

3.15 Field ionization signal as a function of laser frequency between the $n=26$ and 27 sates. The bound population is detected (electrons) after the laser excitation in the presence of the $135-\mathrm{V} / \mathrm{cm}$ microwave field. (a) The integration gate is set to record signal from all bound states. (b) The integration gate is set to record signal only from $n=27$. Vertical dash-dot-dotted and dashed lines are $79 \mathrm{GHz}$ apart from $n=26$ and 27 peaks, respectively. Three sideband peaks are observed for each of these states, and their respective sideband manifolds overlap. Nevertheless, atoms excited at sidebands of $n=27$ stay in $n=27$ : no population transfer between 26 and 27 Rydberg states is observed. . . . . . . . . . . . . . . . .

3.16 Field ionization signal as a function of laser frequency between the $n=29$ and 30 states. The bound population is detected (electrons) after the laser excitation in the presence of $135-\mathrm{V} / \mathrm{cm}$ microwave field. (a) The integration gate is set to record signal from all bound states. (b) The integration gate is set to record signal only from $n=30$. Vertical dash-dot-dotted and dashed lines are $79 \mathrm{GHz}$ apart from $n=29$ and 30 peaks, respectively. Two pronounced sideband peaks are observed for each of these states, as their respective sideband manifolds overlap. Atoms excited at sidebands of $n=30$ stay in $n=30$ : no population transfer between 29 and 30 Rydberg states is

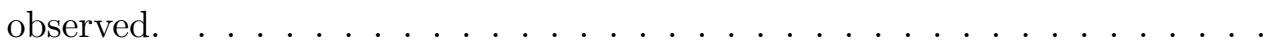


3.17 A fraction of atoms ionized as a function of microwave field amplitude when the laser is tuned to (a) $n=36$ and (b) $n=38$. Electrons are detected, and a percentage of population left bound after the microwave pulse is subtracted from $100 \%$. Laser polarization is parallel (squares) and perpendicular (circles) to the microwave polarization. The data are taken in the presence of small (filled figures) and large $4.5-\mathrm{V} / \mathrm{cm}$ static field (open figures). Ionization thresholds observed at $15-\mathrm{GHz}$ ionization of $\mathrm{Na}$ are shown by a dotted vertical line (Inglis-Teller limit of $1 / 3 n^{5}$ ) and a dashed vertical line (field ionization at $\left.1 / 9 n^{4}\right)$. The ionization field of $n=36$ decreases substantially with the static field due to an enhanced probability of making a transition to higher Rydberg states, while ionization of $n=38$ does not depend significantly on the static field because the resonance conditions are not satisfied due to the spacing between $n=38$ and higher Rydberg states. . . . . . .

3.18 A fraction of population left in bound states after the $120-\mathrm{V} / \mathrm{cm}$ microwave pulse of varied width. Atoms are excited to $n=36$ (filled squares) and $n=38$ (filled circles) in the presence of a small $10-\mathrm{mV} / \mathrm{cm}$ and large $4.5-\mathrm{V} / \mathrm{cm}$ static field (open squares and circles respectively). Data points of $n=36$ are fitted with exponential decay curve of first order as $A e^{-x / t}+B$. A curve taken with $4.5-\mathrm{V} / \mathrm{cm}$ static field decays twice as fast as a curve taken with a small static field (740 and $1700 \mathrm{~ns}$ respectively). In contrast to the ionization experiment at $15 \mathrm{GHz}$, ionization fields depend strongly on the width of the pulse. . .

3.19 Microwave fields as a function of laser tuning for $50 \%$ and $10 \%$ ionization. Microwave pulse width is $500 \mathrm{~ns}$. Vertical dashed lines on the graph correspond to the energies of Rydberg states for which the classical Kepler frequency equals $5 w-0.5 w \ldots \ldots \ldots \ldots \ldots$

3.20 An expanded view of the $50 \%$ ionization fields in the $\Omega>1$ regime. Up to $-500 \mathrm{GHz}$, our result is in agreement with a prediction of Jensen et al. (Eq. 1.16) [25] (red dotted line) that the average ionization field does not depend on $n$. That prediction, however, is not intended to reproduce the structure we observe. As shown by the vertical dashed lines spaced by $79 \mathrm{GHz}$, the structure in the ionization fields is at the microwave frequency. Vertical arrows show the first $(W \sim 0.5 \omega)$ and the fourth maximum $(W \sim 3.5 \omega)$ of the structure below the IL. The horizontal lines show the first $(W \sim \omega)$ and the forth minima $(W \sim 4 \omega) . \ldots \ldots \ldots \ldots$

3.21 Extracted single photoionization rates compared to the Fermi's Golden Rule predicted rates when the laser is tuned $80 \mathrm{GHz}$ below the IL as shown by the horizontal arrow in Fig. 3.20. The experimental data points are fit with $A x$. The observed rates are linear in power and are in a good agreement with the predicted values. . . . . . . . . . . . . . . .

3.22 An oscilloscope trace taken when the laser is tuned one, two, three, four MW photons from the IL with the microwave field off (broken lines), and when atoms are excited $4 \mathrm{MW}$ photons from the IL and exposed to $60-\mathrm{V} / \mathrm{cm} 30-\mathrm{ns}$ microwave pulse (solid line). The microwave field populates states three, two, and one photons below the IL where atoms photoionize. . . . . . . . . 
3.23 An oscilloscope trace taken when the laser is tuned half a MW photon from the IL with the microwave field off (dash-dotted line) and $3 \mathrm{MW}$ photons below it with the microwave field off (dashed line), after $30 \mathrm{~V} / \mathrm{cm} 30$-ns pulse (dotted line), and after $60 \mathrm{~V} / \mathrm{cm} 30$-ns pulse (solid line). Both energies are marked by vertical arrows in Fig. 3.20. The microwave field does not populate states between the initial state and states half a photon below the IL. Rather a large fraction of population is found in the extremely high-lying states where atoms can survive long and large amplitude microwave pulses. The vertical dashed line shows the position of the peak corresponding to the zero-field states tuned 1.5 microwave photons below the IL. Bumps on the falling edge of the peaks are due to the electrical ringing, and do not correspond to the signal from atoms excited 1.5 or 2.5 photons below the limit. . . . . . . . . . . . . . . .

4.1 Experimental timing diagram for the investigation of microwave spectrum in Li by a $38.3-\mathrm{GHz}$ microwave field . . . . . . . . . . . . . .

4.2 Field ionization signal as a function of laser frequency tuning, in terms of energy relative to the ionization limit (IL), after exposure to a microwave pulse of amplitude: $0 \mathrm{~V} / \mathrm{cm}, 16 \mathrm{~V} / \mathrm{cm}, 36 \mathrm{~V} / \mathrm{cm}$, and $45 \mathrm{~V} / \mathrm{cm}$. The horizontal axis is calibrated by the etalon and optogalvanic signals. . . . . . . . . .

4.3 Field ionization signal as a function of laser frequency tuning. $90-\mathrm{V} / \mathrm{cm}$ microwave field is on during the laser excitation. A uniform structure of peaks appears above the IL and continues smoothly across the limit down to energies where $\Omega=1 / 5$. As shown by the vertical dotted lines spaced by $38.34 \mathrm{GHz}$, the structure is at the microwave frequency. . . . . . . . .

4.4 Field ionization signal as a function of laser frequency tuning. Top trace: excitation in zero-field (scaled by a factor of 5); bottom scan: excitation in the presence of the $90-\mathrm{V} / \mathrm{cm}$ field. A break is shown on the horizontal axis for convenience. . . . . . . . . . . . . . . .

4.5 Field ionization signal as a function of laser frequency, after exposure to a microwave pulse of amplitudes: (a) $0 \mathrm{~V} / \mathrm{cm}$, (b) $36 \mathrm{~V} / \mathrm{cm}$, (c) $45 \mathrm{~V} / \mathrm{cm}$, (d) $63 \mathrm{~V} / \mathrm{cm}$, and (e) $90 \mathrm{~V} / \mathrm{cm}$. A 50-ns integration gate is used to detect only atoms bound within $75 \mathrm{GHz}$ of the IL. The zero field spectrum is scaled by a factor of $1 / 3$ to fit the graph. Horizontal dotted lines show the zeroes of the corresponding spectra. The traces are offset by the square root of the microwave field. The broken line connects the onsets of the peaks in the different spectra, and the energy of the onset obeys the relation $W=-4 \sqrt{E}$.

4.6 Field ionization signals as a function of laser frequency for excitation in the presence of a microwave pulse of amplitude of $90 \mathrm{~V} / \mathrm{cm}$. (a) The total photoabsorption spectrum when detecting all energies above $n=43$. (b) Spectrum obtained when detecting only the high-lying states. The total photoabsorption spectrum is scaled by a factor of eight to fit the graph. Horizontal dashed lines show the zeroes of the corresponding spectra. The total photoabsorption spectrum also presents the $38 \mathrm{GHz}$ structure at energies below $-1500 \mathrm{GHz}$ since only atoms transferred to higher lying states are detected. 
4.7 Field ionization signal as a function of laser frequency tuning after exposure to a microwave pulse of $90 \mathrm{~V} / \mathrm{cm}$ amplitude for different static fields in the interaction region: (a) $9 \mathrm{mV} / \mathrm{cm}$, (b) $17 \mathrm{mV} / \mathrm{cm}$, (c) $25 \mathrm{mV} / \mathrm{cm}$, (d) 40 $\mathrm{mV} / \mathrm{cm}$, and (e) $55 \mathrm{mV} / \mathrm{cm}$. A break is shown on the horizontal axis for convenience. Horizontal dashed lines show the baselines of the corresponding spectra. Vertical blue lines intersect the horizontal baselines at points to which the ionization limit is depressed due to $W=2 \sqrt{ } E$ for the corresponding static field $E$. The double headed arrow $19 \mathrm{GHz}$ long is the difference in the depression of the limit by 40 and $9 \mathrm{mV} / \mathrm{cm}$ fields. It is apparent that peaks in the microwave structure also shift by $W$, irrespective of the laser frequency. Increasing the static field destroys the microwave structure, and the $55-\mathrm{mV} / \mathrm{cm}$ spectrum shows only noise, with no bound population detected.

4.8 Field ionization signal as a function of laser frequency tuning. $90-\mathrm{V} / \mathrm{cm}$ microwave field is present during the laser excitation and is on for $30 \mathrm{~ns}$ (a), $330 \mathrm{~ns}$ (b), and $530 \mathrm{~ns}$ (c) after the last laser pulse. Only the extremely highlying states are collected with a narrow integration gate. Horizontal dashed lines show zero of the corresponding spectrum. As shown by the vertical dotted lines spaced by two MW photons, the microwave structure is present in all traces, even when atoms spend $500 \mathrm{~ns}$ in the microwave field after the laser pulse ends. . . . . . . . . . . . . . . . .

4.9 Field ionization signal as a function of microwave pulse duration after the laser excitation. The microwave pulse amplitude is $90 \mathrm{~V} / \mathrm{cm}$, and the laser frequency is fixed and tuned to (a) two mw photons above the ionization limit and (b) 60 photons below it. Only the very high-lying states are collected with a 50-ns gate. The lifetimes exhibit an initial rapid decay followed by a very slowly decaying tail. The solid lines represent fits to the form $A e^{-x / t}+c$. According to the fit, the lifetimes of decaying exponentials are 68(10) and 107(10) ns, respectively. . . . . . . . . . . . . . . .

4.10 Extracted lifetimes of atoms in the high-lying states excited in the presence of the $90-\mathrm{V} / \mathrm{cm}$ field versus the laser tuning. The lifetimes of the fast decay, on average, fall between 60 and $90 \mathrm{~ns}$ for all laser tunings. . . . . . . . .

4.11 Field ionization signal as a function of the laser frequency, in terms of energy relative to the ionization limit. Electrons are detected, and the FIP amplitude is lowered to ionize only the high-lying states within $60 \mathrm{GHz}$ of the IL. The microwave pulse starts before the first laser and is on for $30 \mathrm{~ns}$ after the last excitation laser with 15-ns fall time. A structure of peaks is observed with each peak spaced by a microwave photon of $79 \mathrm{GHz}$. The vertical lines are $79 \mathrm{GHz}$ apart from the first peak below the IL which is depressed by $12 \mathrm{GHz}$ due to the static field of $3-\mathrm{mV} / \mathrm{cm}$ and is ponderomotively shifted to higher energy by $\sim 10 \mathrm{GHz}$. The classical Kepler frequency equals the microwave frequency at $-1700 \mathrm{GHz}(n=44)$. The large peak at $-2300 \mathrm{GHz}$ is due to a two photon excitation. 
4.12 Field ionization signal as a function of the laser frequency between $n=29$ and 30. Electrons are detected, and we used a large FIP with amplitude of $800 \mathrm{~V} / \mathrm{cm}$ to detect all bound states with $n>25$ (a) and a small FIP to detect only the high-lying states within $60 \mathrm{GHz}$ of the IL (b). The top spectrum yields two peaks at frequencies resonant with zero-field Rydberg states and additional peaks corresponding to sidebands spaced an integral number of microwave photons from them. It is clear that the number and position of peaks of spectra are different. Peaks in (a) are always spaced by a multiple of $79 \mathrm{GHz}$ from the corresponding Rydberg state, while the structure of peaks of spectrum (b) is always from the IL. . . . . . . . .

4.13 Field ionization signal as a function of the laser frequency in the presence of $4 \mathrm{mV} / \mathrm{cm} \mathrm{(a),} 14 \mathrm{mV} / \mathrm{cm} \mathrm{(b),} 36 \mathrm{mV} / \mathrm{cm} \mathrm{(c),} \mathrm{and} 57 \mathrm{mV} / \mathrm{cm}$ (d) static fields. Only electrons from the high-lying states within $60 \mathrm{GHz}$ of the IL are detected. The whole structure shifts by the same amount the IL is depressed. Less signal is observed at higher static fields, and at $75 \mathrm{mV} / \mathrm{cm}$ no signal was detected. The vertical lines are $79 \mathrm{GHz}$ apart from the first peak below the IL of the spectrum taken at the smallest static field. Spectra are vertically shifted to fit on the same graph. . . . . . . . . . . . . . . .

4.14 Field ionization signal as a function of the laser frequency in the presence of $3-\mathrm{mV} / \mathrm{cm}$ static field. Only electrons from the high-lying states within 60 $\mathrm{GHz}$ of the IL are detected. The microwave pulse is on for 250 ns (a), 1000 ns (b), and $1700 \mathrm{~ns}$ (c) after the last excitation laser. Even after spending more than $1.5 \mu \mathrm{s}$ in the strong microwave field, a small number of atoms is detected in the high-lying states. For convenience, values of scaled frequency $\Omega$ are shown for some laser tunings. . . . . . . . . . . . . .

4.15 Extracted lifetimesof the extremely high-lying states as a function of the laser frequency for the $80-\mathrm{GHz}$ microwave field with $170-\mathrm{V} / \mathrm{cm}$ amplitude. At each binding energy, a decay curve was fitted with $A e^{-x / t}+B$ and a lifetime $t$, corresponding to the fast exponential decay, was measured. Lifetimes of peaks in the region $\Omega>1$ are, on average, the same and equal to $150 \mathrm{~ns}$. Near $\Omega=1$ lifetimes increase before reaching a value of $260 \mathrm{~ns} . \quad$. . . . . .

4.16 FWHM of peaks in the spectrum of the high-lying states as a function of the laser frequency for the $80-\mathrm{GHz}$ microwave field with $170-\mathrm{V} / \mathrm{cm}$ amplitude. Widths of peaks from several different spectra taken at identical conditions were measured. The $10-\mathrm{GHz}$ laser linewidth was deconvoluted from it using the fact that both the laser distribution and peaks can be represented as gaussians. The inset in the graph shows one of the peaks of the spectrum fitted to a gaussian and a lorentzian distribution with corresponding fit parameter and the FWHM. Peaks below $\Omega=1$ are narrower than those above the IL which means that more microwave cycles are required to drive the population to the high-lying states when the laser is tuned in that region. . 
5.1 Field ionization signal as a function of energy for three microwave field amplitudes. (a) $0 \mathrm{~V} / \mathrm{cm}$, in which case two different gates were used, a wide gate to accept the signal from all Rydberg states of $n \geq 37$ (solid line) and a 50 -ns gate to detect only atoms within $75 \mathrm{GHz}$ of the IL (dashed line); (b) $36 \mathrm{~V} / \mathrm{cm}$, a spectrum recorded with the 50-ns gate; (c) $90 \mathrm{~V} / \mathrm{cm}$, a spectrum recorded with the 50-ns gate. In (b) and (c) we see non-zero signal within the narrow gate, when the laser is tuned to the Rydberg states from which no signal is detected with the same gate when the MW field is off. Not only the expected microwave structure is present near the IL, but also a large fraction of population is observed due to a transfer from the bound Rydberg states to the extremely high-lying states, even when the laser is tuned to a state with $n$ as low as $30 . \ldots \ldots \ldots \ldots \ldots$

5.2 Fraction of atoms surviving the MW field as a function of its strength when the laser frequency is tuned to four different Rydberg states $(n=46,65$, 93, and 135). All bound states are detected (wide gate). For each of these binding energies the $50 \%$ ionization field is almost the same, as predicted by Eq. 1.16. For all of the states minimal further ionization is produced by raising the microwave field above $60 \mathrm{~V} / \mathrm{cm} . \ldots \ldots \ldots$

5.3 Fraction of atoms surviving the microwave field as a function of its strength when the laser frequency is tuned to four different Rydberg states, the same states as shown in Fig. 5.2, $n=46,65,93$, and 135. Only the extremely highlying states are detected (narrow 50-ns gate). The field ionization signals at microwave fields above $60 \mathrm{~V} / \mathrm{cm}$ in Fig. 5.2 are due to atoms being left in

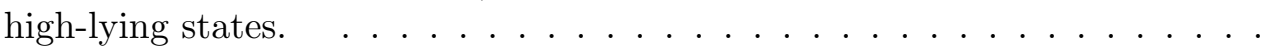

5.4 Fraction of atoms in the high-lying states surviving the microwave field as a function of the width of the microwave pulse for several field amplitudes. The laser is tuned 40 microwave photons below the IL $(n=46)$, but only the extremely high-lying states are detected (narrow 50-ns gate). 90 and $45-\mathrm{V} / \mathrm{cm}$ curves are fit to $A e^{-x / t}+B$. Lower field curves show that many microwave cycles are required to bring atoms to the limit when the laser is tuned far from it. . . . . . . . . . . . . . . . .

5.5 Final state distribution as a function of the MW field amplitude when the laser frequency is tuned to four different Rydberg states. The distributions are gray scale representations of time resolved field ionization signals. For convenience the vertical scale is shown both as time and field in the FIP. The FIP starts at $20 \mathrm{~ns}$. Population is always found in extremely high-lying states at fields when a large fraction of atoms is ionized. Redistribution of the population at lower microwave fields occurs, but atoms are in no case continuously distributed between the initial state and the IL. . . . . . . . . 
5.6 Field ionization signal from the high-lying states of Li after exposure to a $300-\mathrm{V} / \mathrm{cm}$ microwave pulse as a function of the laser frequency, in terms of energy relative to the ionization limit. The inset shows the spectrum in the vicinity of the ionization limit obtained with the same narrow integration gate when no MW field is present. If the ionization spectrum is measured under identical circumstances (detecting ions produced by the mw pulse), a similar trace is obtained. In other words, we detect the high-lying states when the laser is tuned to any Rydberg state that can be ionized by the microwave field. . . . . . . . . . . . . . . .

5.7 Microwave ionization of the Li $73 p$ state.(a) Fraction of atoms surviving the MW field as a function of its strength detecting electrons from all bound states of $n \geq 55$ (circles) and fraction of atoms apparently ionized by the MW field as a function of its strength observed by detecting ions with the positive FIP (squares). The apparent microwave ionization signal collected with the positive FIP is due to both microwave ionization and field ionization of atoms in state of $n \geq 90$. Because the high-lying states are present in both signals, they are not complementary. (b) Fraction of atoms detected in the high-lying, $n \geq 250$, subsequent to the microwave pulse. Fraction of atoms in such high-lying states is also estimated from graph (a) as: ionization probability minus (100\% minus survival probability) for any given MW power (squares). When the MW field exceeds $30 \mathrm{~V} / \mathrm{cm}$, the only bound population remains is in states with $n>250 . \ldots \ldots \ldots$

5.8 Relative number of atoms in the high-lying states with $n>250$ after the MW pulse as a function of static field in the interaction region. The laser frequency is tuned to two states: $\mathrm{n}=58$ and 24 . The inset shows a relative number of atoms in the high-lying states as a function of bias voltage on one of the plates after excitation of the $\mathrm{Li} 58 p$ state and exposure to a $6-\mathrm{V} / \mathrm{cm}$ microwave pulse. The main figure show the relative numbers of surviving high-lying atoms as a function of the static field for the Li $24 p(-)$ and $58 p$ states. The microwave fields are 215 and $6 \mathrm{~V} / \mathrm{cm}$, respectively. In both cases these microwave fields are just above the ionization threshold fields. The two curves are almost identical, and for both states no bound atoms are detected when the static field is about $35 \mathrm{mV} / \mathrm{cm}$, a field that ionizes states as low as

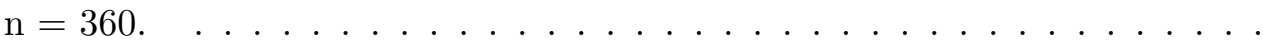

5.9 Fractional apparent ionization for the Na $24 f$ (filled circle), $30 f$ (filled triangle), and $40 f$ (filled square) states obtained with a positive FIP. The signals contain both microwave ionization and field ionization of high-lying states with $n>250$. Vertical lines correspond to the $50 \%$ ionization threshold of $|m|=0$ and 1 states measured by Pillet et al. [13] Fractional high-lying state signals obtained for the $24 f$ (open circles), $30 f$ (open triangles), and $40 f$ states (open squares) obtained with a negative FIP and a narrow integration gate. As in Li, the high-lying states begin to be observed at the field at which microwave ionization starts, and in all cases roughly $15-20 \%$ of the atoms are left in the high-lying states. . . . . . . . . . . . . . 
5.10 The static field dependence of the high-lying state signal of the $30 f$ after exposure to a $120-\mathrm{V} / \mathrm{cm}$ microwave pulse. The high-lying state signal observed by detecting electrons with a negative FIP (solid line). Just as for Li, no bound atoms are detected in the high-lying states when the static field is about $35 \mathrm{mV} / \mathrm{cm}$, a field that ionizes states as low as $\mathrm{n}=360$. Vertical dashed lines at $1 \mathrm{~V} / \mathrm{cm}$ show the static field that was, most likely, present in previous ionization experiments that precluded an observation of the high-lying states. 119

5.11 Field ionization signal as a function of the laser frequency between $n=26$ and 33. Ions produced by the microwave field are detected, as the FIP amplitude is not sufficient to ionize these states (only those with $n>50$ ), so a microwave ionization spectrum is detected. After the laser excitation, atoms are exposed to a $170-\mathrm{V} / \mathrm{cm} 500-\mathrm{ns}$ microwave pulse. It is evident that microwave field ionized substantial number of atoms from all excited Rydberg states. . . . . . . . . . . . . . . . . .

5.12 Field ionization signal as a function of the laser frequency between $n=26$ and 33. Microwave field amplitude is $170 \mathrm{~V} / \mathrm{cm}$. Electrons are detected, and the FIP amplitude can ionize states within $50 \mathrm{GHz}$ of the IL, so a survival probability from the extremely high-lying states is detected. Vertical arrows indicate energies of zero-field Rydberg states where no peaks are present in the measured spectrum. Vertical dotted lines are spaced by a microwave photon from the IL. . . . . . . . . . . . . . . . . .

5.13 Field ionization signal as a function of the laser frequency between $n=26$ and 33. Microwave field amplitude is $170 \mathrm{~V} / \mathrm{cm}$, and the frequency is 79.05 $\mathrm{GHz}$ (a) and $79.65 \mathrm{GHz}$ (b). Electrons are detected, and the FIP amplitude can ionize states within $50 \mathrm{GHz}$ of the IL, so a survival probability from the extremely high-lying states is detected. Vertical arrows indicate energies of zero-field Rydberg states where no peaks are present in the measured spectrum. Vertical dotted lines are spaced by a microwave photon from the IL. Vertical lines in (a) are off by approximately $30 \mathrm{GHz}$ from those in (b). Consequently, different zero-field states are in resonance with the IL, and a different set of peaks is observed. . . . . . . . . . . . .

5.14 Field ionization signal as a function of the laser frequency for $170-\mathrm{V} / \mathrm{cm}$ 79.05-GHz microwave field. The first peak at $-3910 \mathrm{GHz}$ is at the frequency corresponding to $n=29$. The static field is increased to shift the IL by 25 GHz. Electrons are detected, and the FIP amplitude can ionize states within $50 \mathrm{GHz}$ of the IL, so a survival probability from the extremely high-lying states is detected. Vertical dotted lines are spaced by a microwave photon from the IL. Non-zero signal is detected only if the initial state is in resonance with the IL for the whole range of laser tunings. . . . . . . . . . . .

5.15 Field ionization signal as a function of the laser frequency for $170-\mathrm{V} / \mathrm{cm}$ 79.05- GHz microwave field. The static field is increased to shift the IL by $25 \mathrm{GHz}$. Ions are detected. FIP ionizes atoms with $n \sim 90$. Vertical dotted lines are spaced by a microwave photon from the IL. . . . . . . . . . . . 


\section{Chapter 1}

\section{Introduction}

This work is devoted to investigating an interaction of Rydberg atomic states with external microwave fields. This subject has been studied substantially during previous years in our laboratory and many other research centers around the world. Our contribution is an exploration of such interactions in a presence of very small static fields on the order of 5 $\mathrm{mV} / \mathrm{cm}$. Conducting an experiment with well-controlled static fields showed new features of microwave ionization at frequencies below $40 \mathrm{GHz}$ not observed in previous experiments. Also, a novel microwave ionization experiment at $80 \mathrm{GHz}$ allowed an observation of ionization mechanisms different from the ones at lower frequencies.

The most astonishing result of this work turned out to be an observation of the extremely high-lying states left bound after long ionizing microwave pulses for a very wide range of binding energies. Such states show extreme stability in the microwave fields and survive the Rydberg electron revisiting the ion core more than once during the microwave pulse. We have conducted experiments two different ways: excitation to a Rydberg state in the presence of strong microwave fields and excitation in zero-field with a subsequent exposure to the microwave pulse. Atoms can be found in the high-lying states in both cases if the initial state is, or can be, shifted in resonance with the ionization limit.

In the current chapter, I will present an overview of Rydberg atom basics and remind the reader about previous work related to this experiment. After that, a detailed explanation of the experimental apparatus can be found. The following three chapters are devoted to discussing the observations of experiments conducted at three different microwave frequen- 
cies: 17, 38, and $80 \mathrm{GHz}$. Chapter 3 describes results of ionization experiments. Chapter 4 discusses the spectra of Rydberg states that are observed when the microwave pulse is on during the laser excitation and the high-lying states are produced for laser excitation both above and below the ionization limit. Chapter 5 investigates a production of the extremely high-lying states found after the strong microwave pulse.

\subsection{Rydberg Atoms}

Rydberg atoms were first introduced by J. Rydberg in 1889, and became a target of extensive investigation with an advent of lasers. An atom in an excited state with a large principle quantum number $n$ is a called Rydberg atom. The simplest example of a Rydberg atom is the Bohr model of $\mathrm{H}$ atom with energy of an excited state:

$$
W=-\frac{k^{2} Z^{2} e^{4} m}{2 n^{2} \hbar^{2}}=-\frac{R y}{n^{2}}
$$

where $\mathrm{k}$ is Coulomb force constant, $\mathrm{Z}$ is an atomic number, e and $\mathrm{m}$ are electron charge and mass respectively. $R y$ stands for the Rydberg constant that can be used to calculate energy of a Rydberg state. $R y=109737 \mathrm{~cm}^{-1}$ for $\mathrm{H}$, but can be expressed in other units as well. Rydberg constants for other atoms can be found in [1].

Eq. 1.1 shows that energy of Rydberg atoms scales as $n^{-2}$ with principle quantum number. Nearly all important properties of Rydberg atoms, in fact, scale proportionally to a power of $n$ which make them remarkable and interesting to study. For example, size of a Rydberg atom scales as $n^{2}$, which means that the valence, or Rydberg, electron is in a big loosely bound orbit around its ion core. Consider an atom with $n=100$. Its radius is $10^{4}$ times bigger than the size of the ground state atom. Moreover, the spacing between Rydberg states decreases with principle quantum number as $n^{-3}$, and so does the classical Kepler frequency of the electron orbiting the core. 


\subsubsection{Atomic units}

When working with Rydberg atoms, it is extremely convenient to use atomic units. The general idea is that all physical constants are made equal unity. Eq. 1.1 than can be rewritten as:

$$
W=-\frac{1}{2 n^{2}}
$$

It is very easy to use formulae that do not contain an enormous number of symbols in it. It is also very simple to convert atomic units back to SI. In order to do so, a quantity in atomic units has to be multiplied by a constant that corresponds to desirable units in SI. For example, a value obtained in Eq. 1.2 has to be multiplied by $6.5761 * 10^{6} \mathrm{GHz}$, if an energy in frequency units has to be obtained, or by $2 * 13.6 \mathrm{eV}$ if it has to be in electronvolts. Some useful conversion constants are listed in the table below, and the full list can be found in $[2]$.

\begin{tabular}{ccc}
\hline Constant & Symbol & Value in SI \\
\hline \hline Mass & $m_{e}$ & $9.1 * 10^{-31} \mathrm{~kg}$ \\
Charge & $\mathrm{e}$ & $1.6 * 10^{-19} \mathrm{C}$ \\
Radius & $a_{0}$ & $5.29 * 10^{-11} \mathrm{~m}$ \\
Energy & $\mathrm{W}$ & $2 * 13.6 \mathrm{eV}$ \\
Frequency & $\omega / 2 \pi$ & $6.576 * 10^{6} \mathrm{GHz}$ \\
Field & $\mathrm{E}$ & $5.142 * 10^{9} \mathrm{~V} / \mathrm{cm}$ \\
\hline \hline
\end{tabular}

\subsubsection{Quantum Defects}

While Eq. 1.2 correctly describes Rydberg energies of $\mathrm{H}$, in all other atoms an ion core

perturbs energy levels. If angular momentum of an atom is small, an orbit of the Rydberg electron is highly elliptical, and the electron's orbit will penetrate the ionic core that consists of the nucleus of charge $Z$ and $(Z-1)$ electrons. There it is exposed to a positive charge greater than one, and its binding energy is increased. So, while different angular momentum states are degenerate in the $\mathrm{H}$ atom, they are not in other atoms unless the angular momentum is large and the core penetration and polarization is negligible. There are several ways to incorporate the effect of the core penetration, the most convenient for 
Rydberg physics being the quantum defect theory. It is assumed that traveling inside the ionic core, the Rydberg electron wavefunction picks up a phase from the non- $\mathrm{H}$ wavefunction. The resulting energy level of a Rydberg state with principle quantum number $n$ and angular quantum number $l$ can be expressed as:

$$
W=-\frac{1}{2\left(n-\delta_{l}\right)^{2}},
$$

where $\delta_{l}$ is a quantum defect that has the following dependence on $n$ :

$$
\delta_{l}=\delta_{0}+\frac{\delta_{2}}{\left(n-\delta_{0}\right)^{2}}+\frac{\delta_{4}}{\left(n-\delta_{0}\right)^{4}}+\ldots
$$

Quantum defects are different for various atoms and angular momentum states. Values of constants of Eq. 1.4 for alkali atoms can be found in Table 16.2 in Rydberg Atoms [1]. Values relevant to this work are given in the table below:

\begin{tabular}{ccccc}
\hline Series & & $\delta_{0}$ & $\delta_{2}$ & $\delta_{4}$ \\
\hline \hline \multirow{2}{*}{${ }^{7} \mathrm{Li}$} & $n s_{1 / 2}$ & 0.399468 & 0.030233 & -0.0028 \\
& $n p_{1 / 2,3 / 2}$ & 0.47263 & -0.02613 & 0.0221 \\
& $n d_{3 / 2,5 / 2}$ & 0.002129 & -0.01491 & 0.1759 \\
& & & & \\
${ }^{23} \mathrm{Na}$ & $n s_{1 / 2}$ & 1.3479 & 0.06137 & \\
& $n p_{1 / 2}$ & 0.8554 & 0.1222 & \\
& $n d_{3 / 2,5 / 2}$ & 0.015543 & -0.08535 & 0.7958 \\
& $n f_{5 / 2,7 / 2}$ & 0.001663 & -0.0098 & \\
\hline \hline
\end{tabular}

\subsubsection{Static Field Ionization}

\section{Hydrogenic ionization}

The fact that an atom in an excited state has an enormous cross section makes it very sensitive to external electric fields that are negligible for a ground state atom. The electric dipole moment is proportional to $r$ and scales as $n^{2}$, producing a strong coupling between the Rydberg electron and the external field of even mild intensity. Thus, an electric field can not only perturb energy levels, but also ionize atoms. 
It is useful first to consider a $\mathrm{H}$ atom in an external electric field. The potential of an electron moving along the field $E$ is modified as

$$
V=-\frac{1}{r}+E r
$$

and is shown in Fig. 1.1. If the Rydberg electron's energy exceeds the three dimensional

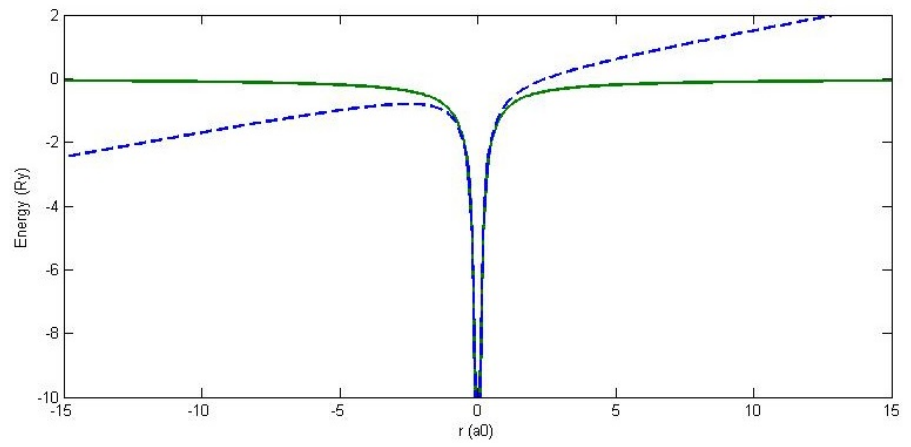

Figure 1.1: Coulomb and modified Coulomb-Stark potential of Eq. 1.5.

potential saddle point, the atom is ionized, and the electron leaves. Consequently, for a given Rydberg state, in order to be ionized, the Rydberg electron energy has to be equal the value of the potential at the saddle point which leads to

$$
E=\frac{W^{2}}{4}=\frac{1}{16 n^{4}}
$$

if the centrifugal potential is absent. Eq. 1.6 is certainly a simplification, as it does not account for the Stark shift that atoms experience in an external electric field. However, it describes correctly an adiabatic ionization of nonhydrogenic atoms in static fields, as will be discussed further.

In the presence of an external electric field, angular momentum $l$ is no longer a good quantum number, and parabolic quantum numbers have to be used. The magnetic quantum number, $m$, is still a good quantum number. In non-zero static field, energy levels are nondegenerate and split from its zero-field values according to

$$
W=-\frac{1}{2 n^{2}}-\frac{3}{2} E\left(n_{1}-n_{2}\right) n,
$$



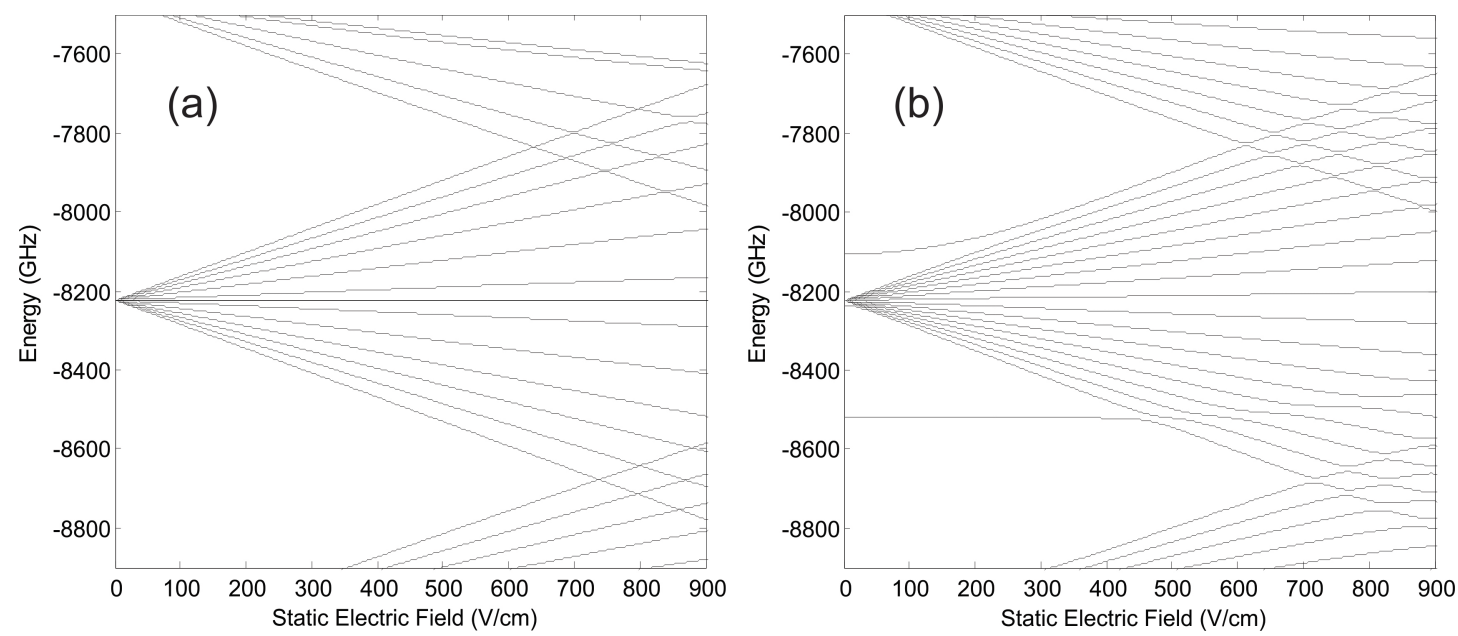

Figure 1.2: Calculated energy level map as a function of static field amplitude for the $n=19,20,21|m|=0$ states of $\mathrm{H}$ (a) and $\mathrm{Na}(\mathrm{b})$. The central state is $n=20$.

where $n_{1}$ and $n_{2}$ are parabolic quantum numbers that are nearly good quantum states that satisfy

$$
n=n_{1}+n_{2}+|m|+1 \text {. }
$$

Fig. 1.2 (a) shows levels of H Rydberg states with $n=19,20,21$ and $|m|=0$ in static field.

It is safe to assume that, for the reddest Stark state of a particular $n$ manifold, $n_{2}-n_{1} \sim$ $n$, and its energy can be expressed as

$$
W=-\frac{1}{2 n^{2}}-\frac{3}{2} E n^{2}
$$

which modifies the ionization threshold for this Stark state to

$$
E=\frac{1}{9 n^{4}}
$$

It is, however, impossible to derive an analytical expression for ionization field of blue states. It is useful to know, though, that a splitting between extreme Stark states of the same $n$ is $\pm 3 n^{2} E / 2$. In atomic units, a spacing between adjacent Rydberg states equals $1 / n^{3}$. Consequently, the bluest state of $n$ manifold crosses the reddest Stark state of $n+1$ 
manifold at a field

$$
E=\frac{1}{3 n^{5}}
$$

That field is often referred to as the Inglis-Teller limit, and will be often encountered throughout this work.

\section{Nonhydrogenic Ionization}

An effect of a finite core in a nonhydrogenic atom is not only the energy shift, as described by quantum defects, but also coupling of extreme Stark states of adjacent manifolds. Fig. 1.2 (b) demonstrates a Stark map of $\mathrm{Na} n=19,20,21$ and $|m|=0$ Rydberg states. The difference with the similar map for $\mathrm{H}$ in Fig. 1.2 (a) is immediately noticeable, as energy levels do not cross. Levels repel each other and form what are called avoided crossings. Depending on how fast the amplitude of a field is swept through an avoided crossing, atoms can stay in the same state or make a transition. If the electric field is changed quickly (diabatically), atoms follow across the avoided crossing, and its Stark state is the same. On the other hand, if it is changed slowly, the crossing is swept adiabatically, and the atom remains in the same adiabatic state passing smoothly from one Stark state to another as other avoided crossings are encountered, until the field is sufficient to ionize the atom.

If the field rises slowly from zero, Rydberg atoms will ionize adiabatically at a field $E=1 / 16 n_{s}^{4}$, where $n_{s}$ is an effective quantum number in the field [1]. Thus, utilizing a slow rising field ionization pulse offers effective and selective way to detect Rydberg atoms. In this experiment, we use it to ionize atoms of $\mathrm{Li}$ and $\mathrm{Na}$, as will be discussed in detail in Section 2.8. However, for a given field pulse rising time and amplitude, for some Rydberg atoms with high $n$, the pulse amplitude is changed too fast, and atoms will ionize diabatically at $E=1 / 9 n^{4}$. Later, in Fig. 3.10, we show a final state distribution of atoms as a function of binding energy where both ionization mechanisms are apparent. 


\subsubsection{Tunneling and Multiphoton Ionization}

In the previous discussion, the energy of Rydberg electron had to exceed the potential barrier modified by the static field. That condition does not have to be satisfied if an oscillating field is considered, and an atom can be photoionized. An essential notion behind the photoionization is that atoms have to absorb one or more photons from the external field to gain enough energy to go over the ionization barrier or tunnel through it.

There are two regimes usually encountered in strong field physics that are, in fact, two limiting cases of the same process: multiphoton and tunneling ionization. In a multiphoton regime, a requirement is on the frequency of the field: an atom absorbs an integer number of photons from the field, and its energy surpasses the ionization potential which leads to ionization. On the other hand, in a tunneling regime, a large field amplitude is needed to put atoms just below the potential barrier but not over it. Then, ionization can happen with an arbitrary number of photons, and there is no requirement on the frequency of the field. To distinguish these two regimes, a Keldysh parameter $\gamma[3]$ is often used in strong laser physics which can be calculated as $\gamma=\sqrt{|W| / 2 U_{p}}$, where $W$ is the binding energy of the atom, and

$$
U_{p}=\frac{E^{2}}{4 \omega^{2}}
$$

is the ponderomotive energy which is the cycle averaged quiver energy of a free electron in an electric field. Also, due to the presence of the oscillating electric field, the Rydberg electron experiences a positive energy shift, as an induced dipole [4], described by Eq. 1.12. In intense laser experiments, in most cases, both an initial state and the continuum limit are ponderomotively shifted by the laser field. In case of mild microwave fields, as in our experiment, only the continuum and the extremely high-lying states are ponderomotively shifted from zero-field values.

The Keldysh parameter is essentially a ratio of the oscillating field frequency to the tunneling frequency through the barrier. Thus, if the Keldysh parameter $\gamma<1$, the tunneling picture is applicable, while $\gamma>1$ is typical for a multiphoton case [5]. Intense laser experiments have been conducted with various laser wavelengths to study both regimes. Using a 
single microwave frequency it is possible to probe both regimes spectrally by changing the binding energy of the initial Rydberg state. This gives a big advantage, as it is possible to continuously move from the multiphoton regime, at lower $n$, to the tunneling regime, at very highly excited states, in a single measurement. For example, for a 17-GHz microwave field at $200 \mathrm{~V} / \mathrm{cm}, U_{p}=370 \mathrm{GHz}$ and $\gamma=1$ at $-740 \mathrm{GHz}$ below the limit $(n=65)$. As the excitation laser frequency can be continuously changed from $n=20$ to above the ionization limit, it is possible to sweep through the multiphoton and tunneling regimes, until the single photon ionization $(W \approx \omega)$ is reached, in one measurement. It is also important to note here that, in both regimes, atoms can absorb more field photons than they need to overcome the barrier. In this case, an excess in energy is converted into kinetic energy of the photoelectron which results in a series of peaks spaced by a field photon if its energy is measured [6]. This effect is called above-threshold ionization (ATI) and has been extensively studied in the past thirty years $[6,7,8]$.

In general, the multiphoton regime does not usually require extremely large field strength, and perturbation theory can be applied to describe an ionization rate. In this limit, Nphoton ionization is an Nth order process [9], while a single photon ionization rate of bound state transition to the continuum should be proportional to $E^{2}$ and described by the Fermi's Golden Rule [10] as

$$
\Gamma=2 \pi|<f| r E|i>|^{2}
$$

where we require the final state $f$ to be normalized per unit energy. We will return to that expression in section 3.4.2, where the expected single photon ionization rates will be compared to the experimental observations obtained for $80-\mathrm{GHz}$ microwave fields.

\subsubsection{Scaled Frequency}

As was mentioned above, microwave experiments provide an opportunity to probe an interaction of Rydberg atoms and strong periodic fields spectrally. In many cases, it is very convenient to express the binding energy of atoms in terms of ratio of the microwave 
frequency to the classical Kepler frequency of Rydberg electron,

$$
\Omega=\omega / w_{k}=\omega n^{3}
$$

called the scaled frequency. This parameter also signifies a relationship between the microwave frequency and the spacing between adjacent states: if $\Omega<1$, the spacing is smaller than the frequency, and the Rydberg electron visits the ion core more than once during one microwave cycle and vice versa. As will be seen further, different regimes are applicable if microwave frequency is larger or smaller than the Kepler frequency. A particular case is when $\Omega=1$, and the Rydberg electron motion can become phase-locked to the microwave field $[11,12]$. For the microwave fields used in the current experiment, $\Omega=1$ at $-1780 \mathrm{GHz}$ $(n=44)$ for $80-\mathrm{GHz}$ microwave field, $-1050 \mathrm{GHz}(n=56)$ for $38-\mathrm{GHz}$ microwave field, and $-617 \mathrm{GHz}(n=73)$ for $17-\mathrm{GHz}$ microwave field.

\subsection{Background}

Before describing the current experiment, it is interesting to look back at previous work relevant to our discussion. I will summarize several studies of microwave ionization of low $n$ $\mathrm{Na}$ and $\mathrm{Li}$ atoms by fields with frequencies below $20 \mathrm{GHz}$ in the $\Omega<1$ as well as theoretical and experimental investigations in the regime where $\Omega \geq 1$. Also, several observations of an excited population left bound after the ionizing pulse in microwave and laser experiments will be presented.

\subsubsection{Microwave Ionization}

First, let's consider experiments investigating microwave ionization of alkali atoms in low scaled frequency regime. P. Pillet and colleagues conducted a fundamental study of ionization of $\mathrm{Na}$ atoms by $15-\mathrm{GHz}$ microwave fields $[13,14]$. They excited $n=18-40 s, p$, and $d$ states of $\mathrm{Na}$ which were later exposed to an ionizing microwave pulse. By measuring ions created in microwave ionization, as shown in Fig. 1.3 for $20 s$, they assessed threshold microwave fields required for ionization of atoms in an excited state. Their results showed 


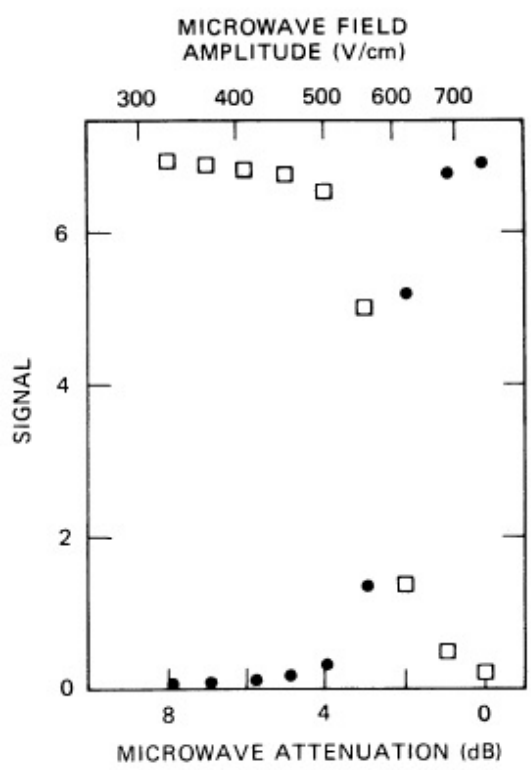

Figure 1.3: Field-ionization signal and microwave-ionization signal for the Na 20s state showing the ionization threshold as a disappearance of the field-ionization signal or the appearance of the microwave-ionization signal with increasing microwave power, from [13].

that $|m|=0$ and 1 states may be ionized with a very low microwave field, $E \sim 1 / 3 n^{5}$, corresponding to the point at which the $n$ and $n+1$ Stark manifolds of principal quantum numbers $n$ and $n+1$ intersect. The $|m|=2$ states, on the other hand, ionize at a significantly higher microwave field, $E=1 / 9 n^{4}$. The difference in the observed threshold fields is attributed to the dynamics of passing from zero field to a high ionizing field. The $|m|=2$ states ionize at classical hydrogenic field due to the fact that Stark levels of $n$ and $n+1$ states cross, and no transitions to higher states occurs. The $|m|=0$ and 1 states ionize by making such transitions at a rate-limiting step corresponding to the Inglis-Teller limit. Thus, the observed microwave ionization thresholds should be the fields at which the transition probability from the highest-energy $n$ Stark state to the lowest-energy $n+1$ Stark state becomes appreciable. This process can be viewed as a ladder climbing mechanism shown in Fig. 1.4. An atom initially excited to the $20 d$ state is brought to the point $\mathrm{A}$ at which the $n=20$ and 21 Stark manifolds intersect. The atom makes a Landau-Zener transition to the $n=21$ Stark manifold and on subsequent microwave cycles makes further upward transitions as atom reaches a sufficiently high $n$ state that the microwave field itself ionizes the atom. Experiments done in other atoms, $\mathrm{He}, \mathrm{Li}$, and $\mathrm{Ba}$, also showed the 


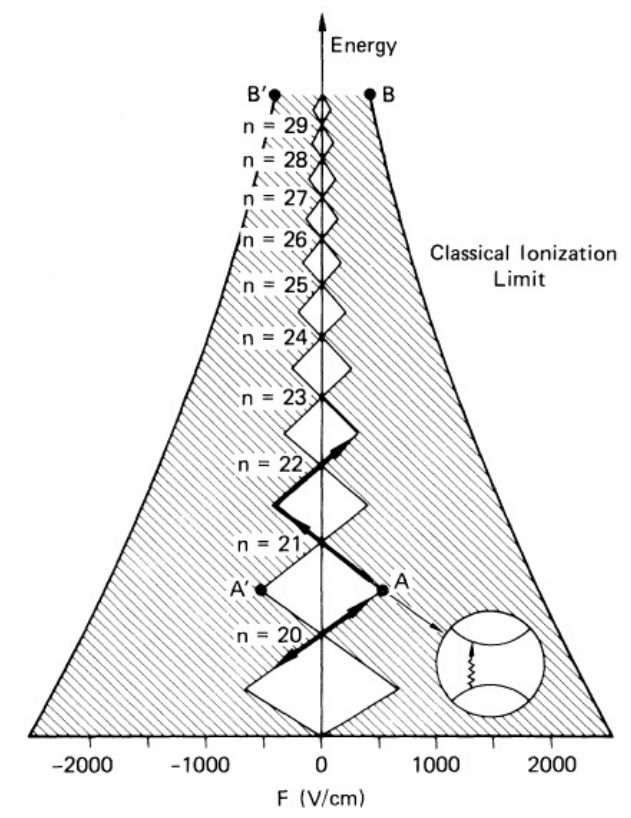

Figure 1.4: Simplified Stark-level diagram for sodium. The bold arrows indicate the first steps leading ultimately to ionization at the classical ionization limit. Updated from [14].

same ionization thresholds $[15,16,17]$. It is important to note that the results of these ionization experiment are rather straightforward: atoms were found either in the initial state or ionized. Other states were not observed to be populated, and no high-lying states were detected. These experiments were conducted in a way, though, that would preclude an observation of highly excited atoms. First, ions were measured, and it was impossible to separate ions from bound atoms in highly excited state from those created in microwave ionization. Also, experiments were done in microwave waveguides, and the static field was not well-controlled. It was, probably, on the order of $1 \mathrm{~V} / \mathrm{cm}$ which was not considered important for ionization experiments.

However, several years after the Na experiment, Pillet et al. showed that a small static field can enhance microwave ionization of Li atoms by lowering threshold fields [18]. It was shown that $1-\mathrm{V} / \mathrm{cm}$ static fields decreased ionization fields of $n=25-50$ Rydberg atoms by an order of magnitude. Lithium atoms in a presence of zero static field ionize hydrogenically at $E=1 / 9 n^{4}$. However, when the static field is added, the ionization field decreases, until it reaches $1 / 3 n^{5}$ limit at a static field $E_{s}=\omega / 6 n^{2}$, which is $2.4 \mathrm{~V} / \mathrm{cm}$ for $n=30$. Atoms of $\mathrm{Li}$, 
except $s$ states, have small quantum defects, and avoided crossings of extreme Stark states investigated in this work are substantially smaller than the detuning between states that is on the order of the 15-GHz microwave frequency. Consequently, ionization does not happen by a sequence of transitions to higher states but by a direct field ionization at $E=1 / 9 n^{4}$. As will be discussed below, the microwave field produces a set of sidebands for each level spaced by the microwave frequency. When the static field is added, these sidebands split due to the Stark effect, the degeneracy is lifted, and a quasicontinuum of states is created. A detuning between $n$ and $n+1$ states is much smaller then, and the transition to higher states can occur which results in lower ionization field.

In an attempt to connect field and photon viewpoints on ionization, in 1984 van Linden van den Heuvell et al. studied the excitation spectrum of $\mathrm{Na}$ Rydberg states in $15-\mathrm{GHz}$ microwave fields [19]. That work was based on prior observation of a spectrum of $\mathrm{H}$ atoms developing sideband states when exposed to a 10-GHz microwave field [15]. Similarly, Pillet et al. had shown a 15.4-GHz sideband spectrum in radiative collisions in $\mathrm{Na}$ [20]. van Linden van den Heuvell et al. also observed an excitation spectra consisting of zero-field state sidebands spaced by the field frequency at different microwave field amplitudes as shown in Fig. 1.5. It was stated that the only effect of the microwave field is to increase a number of sidebands with no observable energy shifts, until the field amplitude reaches the $1 / 3 n^{5}$ level.

At a certain field amplitude, a set of sidebands of one zero-microwave field state overlaps a set of sidebands of the adjacent state. This field is slightly smaller than the field at which the Stark manifolds of these two state intersect. At the Inglis-Teller limit, $E=1 / 3 n^{5}$, sideband sets extend about two peaks into each other. At this point a detuning between extreme Stark states, $\Delta$, is on the order of the microwave frequency, and, if the coupling, $w_{0}$, is comparable to the value of the detuning, or the microwave frequency, a transition from $n$ to $n+1$ Rydberg states occurs which results in a significantly lowered ionization field of $n$ Rydberg state. That condition is satisfied for $|m|=0$ and 1 states of $\mathrm{Na}$ at $15 \mathrm{GHz}$, but not for Li atoms that have smaller quantum defects. Only with the help of static field that produces a quasicontinuum of states to significantly lower the detuning, transitions to 


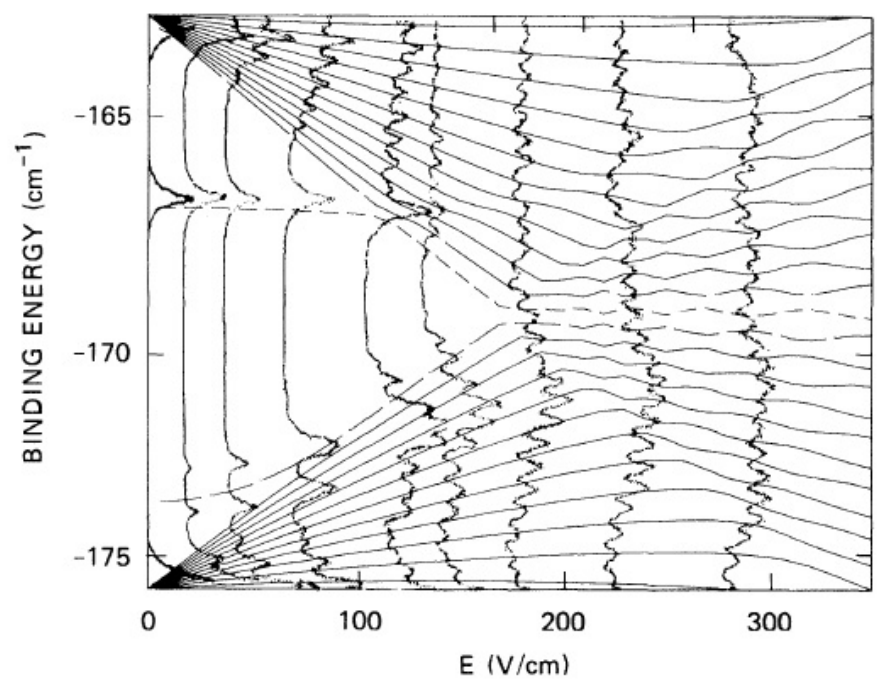

Figure 1.5: Observed spectra in varying strengths of microwave field overlaid on an energy-level diagram of the Na $m=0$ states, from [19]. The spacing between the zero-field $n=25$ and $26 d$ states is $397 \mathrm{GHz}$, and, at the highest field shown, the spacing is filled with a structure of 26 peaks spaced by the microwave frequency.

higher states are possible, as discussed above. In case of $\mathrm{H}$ though, $w_{0}<<\omega$, and only at the occasional resonances where the spacing between states is an integral multiple of the microwave photon would the $n$ to $n+1$ transition and ionization occur. In fact, microwave ionization at the Inglis-Teller limit is not observed in $\mathrm{H}$, but resonances are [21].

Fig. 1.5 also shows that, as the microwave field amplitude is increased above the InglisTeller limit, peaks of different sideband sets coalesce into a single set consisting of a regular structure of peaks spaced by $15 \mathrm{GHz}$ that, in principle, might extend to the continuum. Possibly, atoms in a lower Rydberg state might be able to travel to the ionization limit via transitions through these sidebands. We will test this notion in the microwave ionization experiment at $80 \mathrm{GHz}$ described in section 3.4.2.

In the $\Omega<1$ region, atoms of $\mathrm{H}$ ionize at $E=1 / 9 n^{4}$, but, as the scaled frequency approaches one, it drops below it to approximately $0.04 / n^{4}$ [15]. At this point, transitions to higher states start occurring which lowers the ionization fields. When $\Omega \approx 1$, alkali ionization also becomes similar to the hydrogen ionization, as the avoided crossings become smaller and $|m|=0$ and 1 states ionize like $\mathrm{H}$. Recently, calculations treating $\mathrm{H}$ and alkali atom ionization in this region appeared [22]. Unlike the $\Omega<1$ regime, microwave ionization 
is predicted to be insensitive to the presence of nonzero quantum defects. In other words, ionization of $\mathrm{H}$ should be the same as ionization of alkali atoms.

Surprisingly, in the $\Omega>1$ region, the classical description is no longer applicable, and the experimentally measured fields are higher than the predicted ones [23, 24]. In this region, a localization concept is usually utilized, coming from an Anderson localization model that describes electron transport in one dimensional systems. Jensen et al. [25] proposed that in the $\Omega>1$ regime ionization occurs by a sequence of transitions through excited states. The requirement is that the dipole coupling due to the microwave field equals the average detuning from resonance of the transitions of the sequence. Since both the coupling and the detuning scale as $1 / n^{3}$, this condition is met for all $n$ when

$$
E=2.4 \omega^{5 / 3}
$$

This expression can be understood as follows. The Rabi frequency between two Rydberg states can be written as

$$
\omega_{\text {rabi }}=\left|<n_{i}\right| r E\left|n_{f}>\right|=E \frac{0.4108}{\left(n_{i} n_{f}\right)^{3 / 2} w^{5 / 3}}
$$

using a radial matrix element given by Delone et al. [26]. For highly excited states, $n_{i} \sim n_{f}$, and the maximum detuning is half the spacing between the states. Then, the ratio of Rabi frequency to the detuning is $n$ independent:

$$
a=\frac{\omega_{r a b i}}{\Delta}=\frac{0.4108}{2 w^{5 / 3}} E
$$

Jensen et al. suggests that, when $a \approx 2$, the $\omega_{\text {rabi }}$ is greater than the spacing between adjacent Rydberg states and contains more than one state. Then the coupling of states might extend all the way to the ionization limit, and ionization will occur, which results in the $n$ independent threshold field as expressed by Eq. 1.15.

That prediction was shown to hold in the region $1<\Omega<6$ in $\mathrm{Sr} 50 \%$ ionization investigation [24]. Observations of Gurian et al. also yielded results similar to Jensen's 
prediction [23]. They studied ionization of $\mathrm{Li}$ atoms by $17-\mathrm{GHz}$ microwave fields in the high scaled frequency regime and showed that the $50 \%$ ionization fields, even though confirming on average Eq. 1.15, exhibit a certain structure of peaks spaced by a microwave photon. Moreover, when $\omega \approx 1 / 2 n^{2}$, photoionization should occur, and the perturbation theory should be applicable. Gurian et al. measured single photon ionization rates that turned out to differ from the prediction given by Fermi's Golden Rule (Eq. 1.13) by about a factor of 10. In Chapter 3, we will also compare experimental $50 \%$ threshold values for 17,38 , and 80-GHz fields to Jensen's prediction.

\subsubsection{High-lying states}

When ground state atoms are ionized by an intense laser pulse, Rydberg atoms can be produced as a byproduct [27]. Even when multiple ionization of the atom occurs, it can be accompanied by the production of ionic Rydberg states converging to higher ionization limits [28]. While the notion of producing weakly bound Rydberg states with an intense laser pulse might seem surprising, it can be understood in the following way. The intense laser pulse ejects the electron from the ion core either in a single field cycle, through tunnel ionization, or over many cycles, via multiphoton ionization. In either case, the electron departs from the core with a range of energies into the long range tail of the coulomb potential, where the electron is nearly free, and there is no cycle average energy exchange with the laser field. Above the ionization limit the range of energies appears explicitly in the form of above threshold ionization [6], but the energy range extends below the limit as well. An electron with an energy below the limit is reflected by the coulomb potential and returns to the core in one Kepler period $[27,29,30]$. If the laser pulse is over before the return, the electron obviously remains bound. If the laser pulse is not over, rescattering of the electron from the core can lead to superponderomotive ATI, recombination and high harmonic generation, non sequential double ionization, and nearly elastic scattering. Nearly elastic scattering can result in a very low energy free electron, as observed recently $[31,32,33]$, or in a bound electron $[6,34,35,36,37]$. A striking example of the former is the ionization of He by $1-\mathrm{GV} / \mathrm{cm} 800-\mathrm{nm}$ pulses 30 fs long. It results in $10 \%$ of the atoms' 
being left in states of principal quantum number $n$ between 7 and 15, a rescattering process dubbed "frustrated tunnel ionization" [36, 37]. Essentially, whenever the electron does not gain enough drift energy from the laser pulse after tunneling, it will eventually be captured by the Coulomb field of the ion.

Shuman et al. [38] observed a multiphoton assisted recombination in the presence of a $38.8 \mathrm{GHz}$ microwave field that resulted in the structure of the peaks spaced by the microwave photon below and above the limit. The Rydberg electron looses energy to the field, and such stimulated emission of up to ten microwave photons results in energy transfer from continuum electrons, enabling recombination. The observed spectrum of states is shown in Fig. 1.6. How far the structure extends above the continuum is limited by the maximum

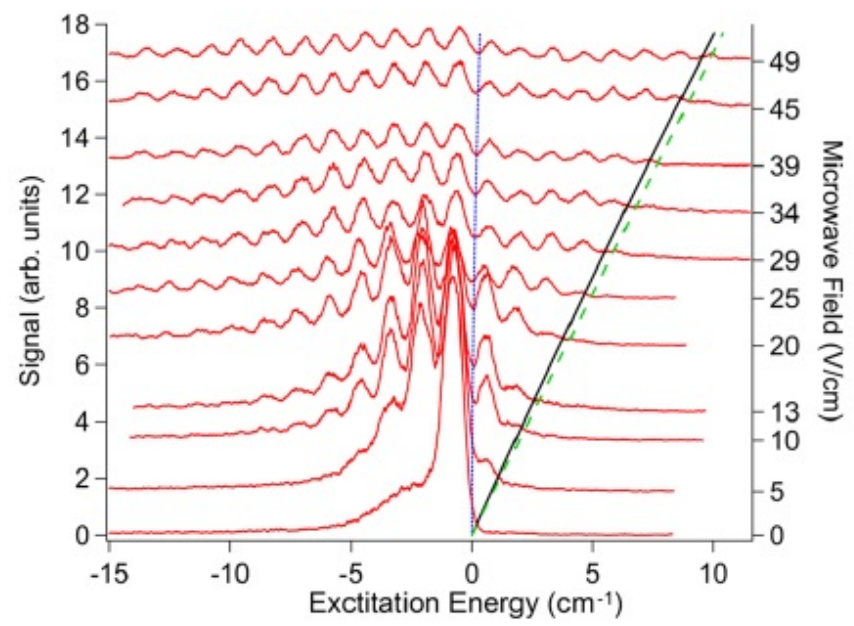

Figure 1.6: Field ionization signal as a function of excitation energy for different microwave fields. The energy scale is relative to the $B a^{+} 6 p_{1 / 2}$ limit. The dotted line and dashed line show the maximum recombination energy predicted by Eqs. 1.18 and 1.19, respectively. Updated from [38].

energy exchange with the microwave field. Often the Simpleman's model is implemented to explain the range of electron energies produced during the above-threshold ionization of atoms in intense laser fields $[39,40]$. In the model, the free Rydberg electron is inserted into the oscillating field, laser or microwave, in a flat potential, and the energy exchange with the field is calculated. When the Coulomb potential is neglected, the electron initial velocity is governed by its binding energy, $v_{0}=2 \sqrt{W_{0}}$, and the time dependent velocity of the electron is only due to the force applied by the field. Then, the work done on the 
electron by the field can be calculated, and the maximum energy extraction by the field is obtained as

$$
\Delta W=\frac{E^{2}}{2 \omega^{2}}
$$

Shuman et al. used the Simpleman's model prediction to compare to the observed results, as shown in Fig 1.6 by the dotted line, which did not match it at all. However, as they showed, the Simpleman's model should be modified to include the Coulomb potential so that $v(t)=\sqrt{2 / r(t)}$ which represents a more realistic situation encountered in the experiment. Then, Eq. 1.18 has to be modified, and the maximum energy transfer at a particular phase of the field is

$$
\Delta W=\frac{3 E}{2 \omega^{2 / 3}}
$$

matching the experimental observations, as shown is Fig. 1.6 by the dashed line.

Gurian et al. [23], in the measurement of highly excited state spectrum created in the presence of the microwave field, showed a structure of peaks spaced by the microwave photon which, apparently, belonged to the extremely high-lying states. This result was obtained with long microwave pulses but small static fields that allowed the observation. His measurements, though, only concerned a regime when $\Omega>>1$. Similarly, Zhao et al. [41] examined both experimentally and theoretically the effect of trains of short unipolar pulses with an added bias field. In the experiments, with trains of up to 40 pulses, they found atoms were most likely to be found in high-lying states subsequent to the pulse train if the bias field resulted in a time average field of zero.

In earlier experiments the production of highly excited states in the recombination of electrons with ions induced by half cycle pulses and microwave fields has been reported [38, $42,43]$. These are essentially rescattering processes, rather similar to frustrated tunnel ionization. In sum, with respect to the production of highly excited states, Rydberg atoms in microwave fields are similar to ground state atoms in strong laser fields. Until our work on $38-\mathrm{GHz}$ microwave ionization presented here, there was only one measurement which showed the production of high-lying states, but in that experiment atoms initially in states of $n \cong 80$ were exposed to a five cycle $11.5 \mathrm{GHz}$ pulse, which resulted in as much as $35 \%$ 
of the population's being left in states of $n>120$ [44]. In all other experiments, with microwave pulses thousands of field cycles long, after the microwave pulse population was apparently either in the initial state or ionized [14]. No population was found in intermediate states.

To conclude this discussion, atoms have been detected in high-lying states subsequent to exposure to short microwave pulses containing only a few field cycles [44] and trains of up to 40 unipolar pulses with a bias field [41]. However, they have only been observed with long pulses when starting from very high-lying states [23]. They have not been observed before in microwave ionization of low-lying states. Results of this work, presented in Chapters 4 and 5 , show that the high-lying states are observed even when $\Omega<1$ if the initial state can be shifted in multiphoton resonance with the ionization limit. 


\section{Chapter 2}

\section{Experimental Apparatus}

The experimental setup described below was built in order to study an interaction of alkali Rydberg atoms with microwave electric fields. It can be divided into four main parts: vacuum apparatus where the experiment is conducted, optical system to excite Rydberg states of atoms, microwave system to produce the microwave field, and data acquisition system.

The essence of the experimental technique is following: ground state alkali atoms in a thermal beam are excited through a sequence of optical transitions to a Rydberg state at an antinode of a Fabry-Perot microwave cavity. There atoms are excited to a well-defined Rydberg state either in a presence of a microwave field or are exposed to it after the excitation ends. Later a field ionization pulse (FIP) is applied to ionize excited population and push the resulting charged particles towards a dual microchannel plate detector (MCP). The MCP signal is captured by a gated integrator or oscilloscope and recorded in a computer for further processing. Typically, experiments are conducted in a presence of a small static field on the order of $5-10 \mathrm{mV} / \mathrm{cm}$ that can be increased in a controlled fashion. In what follows, we provide a detailed description of each element necessary to carry out an experiment similar to the one outlined above.

\subsection{Vacuum Apparatus}

In order to conduct experiments on Rydberg atoms, one has to create an environment where the mean free path of particles is substantially large to ensure that excited atoms, 
with geometric cross section scaling as $n^{4}$, do not redistribute from the state populated by the laser to other states due to collisions with a background gas. That condition is satisfied when the background pressure of a chamber where an experiment is conducted is below $10^{-6}$ torr.

\subsubsection{Two-stage Pumping System}

The vacuum system used in our laboratory is standard for atomic beam experiments and consists of a two-stage pumping. First, a mechanical belt-driven pump is required to create a forevacuum pressure about $10^{-3}$ torr in the chamber and connected to it high-vacuum diffusion pump. Such pressure is required for the diffusion pump to start operating, so it can start lowering the pressure in the chamber further only after the mechanical pump creates the forevacuum.

A high-vacuum diffusion pump is capable of lowering the pressure to $10^{-8}$ torr which is low enough to conduct an experiment involving a thermal atomic beam. Such a pump is based on the momentum transfer to the background gas from an oil vapor that is created by heating up an electric boiler at the bottom of the pump. Oil molecules shoot up, reflect from the lid, and push particles in the chamber towards the bottom of the pump. Then, oil condenses on the walls of the pump that are cooled down externally by running water and drips down back to the boiler, while the system gases are removed by the mechanical pump. More details on how that and similar vacuum systems operate can be found in [45]. We use Edwards Diffstak 160/700M with a pumping speed $700 \mathrm{~L} / \mathrm{s}$ (nitrogen). The pump requires $250 \mathrm{~mL}$ of Santovac 5 oil that is heated by an electrical heater that operates at $220 \mathrm{~V}$. The diffusion pump is connected to the vacuum chamber through a butterfly valve and to a backing mechanical Welch 1376 Duo-Seal pump by an Edwards BRV25 Roughing valve. A purpose of the valve is to connect the mechanical pump to a diffusion pump, vacuum chamber directly, or seal it from both. Pressure in the vacuum chamber is measured by a thermocouple gauge that measures down to 0.001 torr and a standard Bayard-Alpert type ionization gauge with a Varian 843 controller. Due to a large number of flanges in the vacuum chamber, multiple items inside it, and a hot atomic vapor, the base pressure during 
an experiment falls short of the capabilities of the diffusion pump and is on average between $5 * 10^{-6}$ and $3 * 10^{-7}$ torr. It appears that lower pressure is not required for the experiments I was conducting as it did not improve or alter the observed results. However, it is important not to exceed the upper limit, as the Rydberg population in very high principle quantum number states may depopulate or ionize.

Atoms of $\mathrm{Na}$, when used in the experiment, were causing additional depopulation of Rydberg atoms due to collisions, as they do not stick to the walls as much as Li atoms do, and, usually, the vacuum chamber could be full of $\mathrm{Na}$ atoms floating around. To decrease the number of atoms in the chamber outside the atomic beam, we used a nitrogen cup that was much colder than the walls of the chamber. Such cup has to be filled with liquid nitrogen from the outside of the chamber several times during the day when an experiment on $\mathrm{Na}$ atoms is conducted. That not only allows a removal of $\mathrm{Na}$ atoms floating around the chamber, but also lowers the base pressure in it.

\subsubsection{Thermal Beam}

In order to create a thermal beam of alkali atoms, a small thin-walled stainless steel oven is used. Such oven is a $0.005 "$ wall tube with $9 / 32$ OD and 5" length clamped from both sides and stuffed with solid pieces of metal of interest. I used lithium and sodium atoms in the current work, both of which are stored in mineral oil. It is necessary to clean the oil before putting the oven in the chamber, and I used liquid ether for that purpose. A small hole, about $2 \mathrm{~mm}$, has to be drilled in the oven, and the metal pieces should be stuffed up to a level just below it. Once the oven is in vacuum, it is resistively heated until $\mathrm{Li}$ or $\mathrm{Na}$ gas in the tube is created. The vapor pressure of the gas in the tube is high, and it escapes the oven through the hole forming a diffusive beam that travels across the vacuum chamber. Typically, I used 25-50 A and 10-40 A currents running trough the oven for Li and $\mathrm{Na}$ atoms respectively. 


\subsubsection{Chamber Maintenance}

A definite advantage of using a diffusion pump for atomic beam experiments is its versatility and lack of rotating mechanical parts or elements producing electrical noise. A small disadvantage, on the other hand, is a contamination of the vacuum chamber by oil that can absorb free atoms of interest flowing around the chamber and charged particles. Such contamination near the interaction region creates surface electric fields that can alter the static field distribution during the experiment. Even though there are usually enough atoms in the oven to run the experiment for 4-6 months without replacing it, I, typically, had to open the chamber about every two months in order to clean the chamber from the inside. The walls, lid, and all surfaces of items inside the chamber have to be cleaned. I used methanol and acetone for general chamber areas and hot water for areas near the oven. It turns out that cleaning the chamber is necessary to conduct experiments involving extremely high-lying states and has to be done regularly.

In order to open the chamber, the diffusion pump has to turned off and sealed from both the roughing mechanical pump and the vacuum chamber. The mechanical pump can be left working if it is also sealed from the chamber that will be subjected to the atmosphere. When all the maintenance is performed on the chamber, a new oven has to be made and put into its position in the chamber right before it is closed. After the lid is closed and tightened, the roughing pump should work on the chamber with the diffusion pump sealed off. Once the pressure in the chamber is about $10^{-3}$ torr, the valve can be switched to let it work on the diffusion pump with the chamber sealed. Then the butterfly valve between the chamber and the diffusion pump can be opened, and the pump turned on. When the pressure in the chamber stops going down, a very small current should be run through the new oven in order to evaporate remnants of the mineral oil. It is recommended to leave it working for at least 12 hours before the experiment can be started. 


\subsection{Optical Excitation System}

To excite Rydberg atoms, a set of lasers is required to drive atomic transitions. In our laboratory, Littman-Metcalf type dye lasers are mostly utilized for that purpose [46]. The main requirements for these lasers, besides the correct frequency, are: short duration, relatively small linewidth $(5-10 \mathrm{GHz})$, and tunability in the range of 10-20 nm. To pump such dye lasers, typically, frequency doubled, Q-switched Nd:YAG lasers with 20-30 Hz repetition rate are used. We, however, implement a $\mathrm{kHz}$ laser system that allows us to record and average 50 times as many data points in the same amount of time. A design and performance of this laser system is described in great detail in a thesis of J. Gurian [47], so here I will only outline the main features of the pump system.

\subsection{1 kHz Pump Laser System}

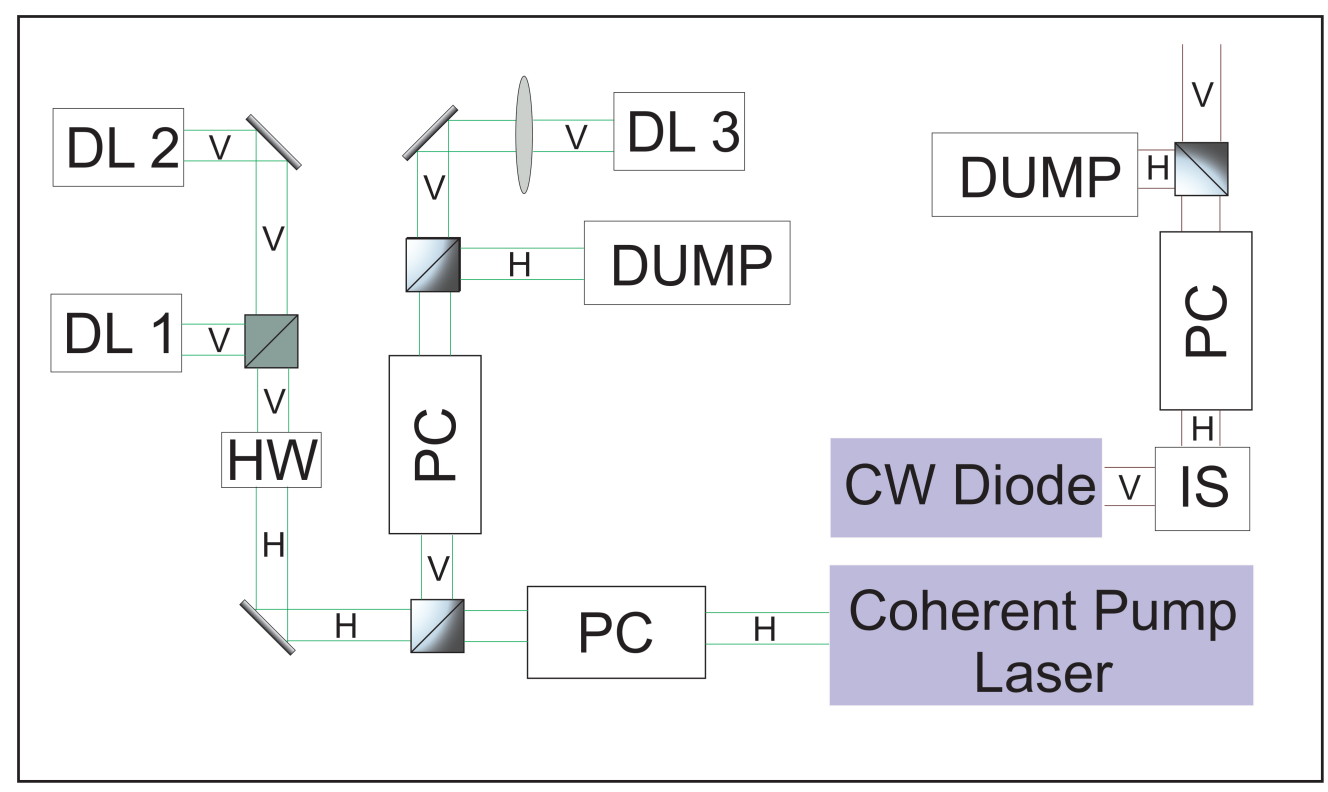

Figure 2.1: Optical diagram of the pump beam. The output pulse of the 527-nm pump beam is 'sliced' by two Pockels cells (PC) resulting in two 20-ns pump beams. The first pulse travels through a half-wave plate (HW), a 50/50 beam splitter and pumps two dye lasers (DL) that drive the first two atomic transitions. Passing through a 1-m converging lens, the second 20-ns pulse pumps another dye laser that drives atoms to a Rydberg state. For convenience, the polarization of laser pulses is shown ( $\mathrm{H}$ for horizontal, $\mathrm{V}$ for vertical). To populate $3 p-3 d$ transition of $\mathrm{Na}$, a cw Toptica diode laser is used. Its output goes through two optical isolators (IS) and is 'sliced' in a Pockels cell to form a step function in such a way that this laser is on immediately after the first dye laser pulse. All output laser beams driving atomic transitions are polarized vertically, unless extra half-wave plates are introduced to rotate the laser polarizations. 
The whole experimental apparatus is triggered from the Coherent Evolution 30 diode pumped solid state, frequency doubled, Q-switched Nd:YLF laser. The laser is run at a $1-\mathrm{kHz}$ repetition rate that can be lowered if required. It produces horizontally polarized $527-\mathrm{nm}$ pulses about $200 \mathrm{~ns}$ wide with approximately $20-\mathrm{W}$ average power. The output of that laser is used to pump three dye lasers that excite atoms to a Rydberg state. That is done with the help of two Pockels cells and polarizing beam splitters that 'slice' a long pump laser pulse into two short 20-ns pump beams. A diagram of an optical system is shown in Fig. 2.1. The output of the pump laser enters the first Pockels cell, and the high voltage is switched about $50 \mathrm{~ns}$ after the beginning of the laser pulse with a 2-ns rise time to change a polarization of the rest of the pulse to vertical. Next, the beam enters a polarizing beam splitter cube that produces two spatially separated beams that have different polarizations. The horizontally polarized 20-ns beam travels through the cube without changing its direction and pumps the first two dye lasers. However, before reaching them its polarization is changed to vertical with a half-wave plate, and the beam is split into two by a $50 / 50$ beam splitter.

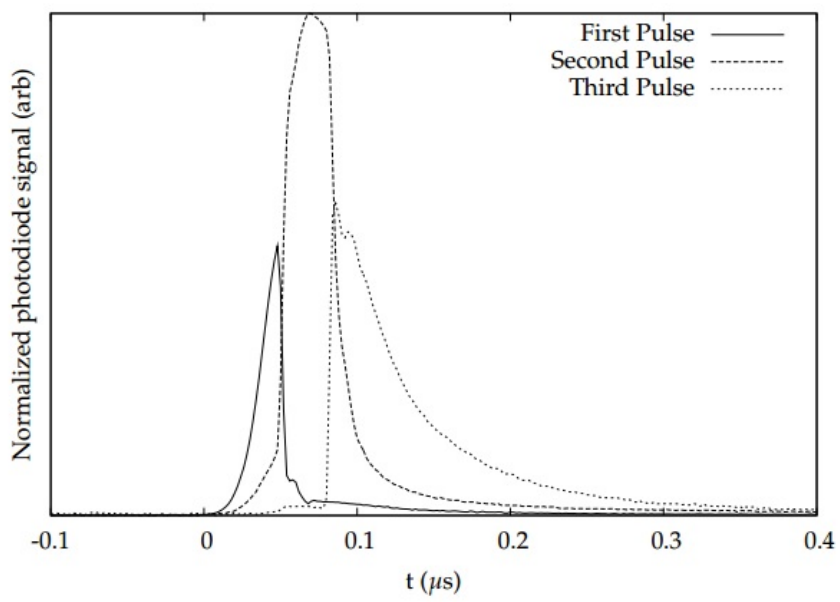

Figure 2.2: Timing of the 527-nm pulses after passing through Pockels cells. The first pulse pumps two first dye lasers, the second one pumps the last transition dye laser, while the long tail is sent to a dump. The picture is updated from [47].

The vertically polarized beam leaves the polarizing beam splitter at a 90 degree angle and enters another Pockels cell that is switched $80 \mathrm{~ns}$ after the beginning of the pulse changing the polarization of the pulse tail back to horizontal. A polarizing beam splitter 
that follows separates the incoming beam into two with the vertically polarized 25 -ns beam traveling straight to pump the third dye laser, while the long tail of the pulse is sent upward into a dump. Fig. 2.2 is updated from J. Gurian's thesis [47] and shows the pump beam pulses after the slicing by two Pockels cells. The first pulse is used to pump dye lasers responsible for two first atomic transitions. The second beam pumps the third dye laser driving a Rydberg transition and starts after the end of the first pump beam as shown in Fig. 2.2. The third pulse consists of the long tail that contains a lot of power and can burn a dye cell. We do not use that beam in the experiment, though it could have been used to pump a dye amplifier if required. An important improvement in the pump setup became a replacement of the avalanche transistor based Marx-bank circuits used to generate halfwave voltage for Pockels cells by LaserMetrics MW4024F power generators. These cheap commercially available $24-\mathrm{V}$ adapters are simple in use, more reliable, and substantially less noisy than the old Marx-bank circuits.

\subsubsection{Atomic Energy Levels}

Li atoms are excited to a Rydberg state via $2 s \rightarrow 2 p \rightarrow 3 s \rightarrow n p$ transitions that require lasers operating on 617.911-nm, 812.846-nm, and 614-620-nm wavelengths, respectively, as shown in Fig. 2.3. The first 20-ns 527-nm Nd:YLF pulse pumps the $2 s-2 p$ and $2 p-3 s$ Littman-Metcalf [46] dye lasers, while the second one pumps the last $3 s-n p$ transition double grating Littman laser [48] with the linewidth of the laser about 5-8 GHz.

Na atoms are excited via $3 s \rightarrow 3 p \rightarrow 3 d \rightarrow n f$ transitions. The first $527-n m$ pulse pumps a $3 s-3 p$ Littman-Metcalf dye laser. The second pump beam is used for a near infrared Littman-Metcalf dye laser that drives the last transition. The linewidth of this laser is about 10-12 GHz. Amplified spontaneous emission of the second laser drives the $3 p-3 d$ transition, while the output of the oscillator populates an $n f$ Rydberg state. The spontaneous emission can effectively drive the $3 p-3 d$ transition, but is not strong enough to excite an observable number of atoms to Rydberg states or to photoionize them. To populate the $3 d$ level with more atoms, we use a Toptica DL100 infrared diode laser to drive the transition from $3 p$. Fig. 2.1 shows an optical path diagram for that laser as well. 


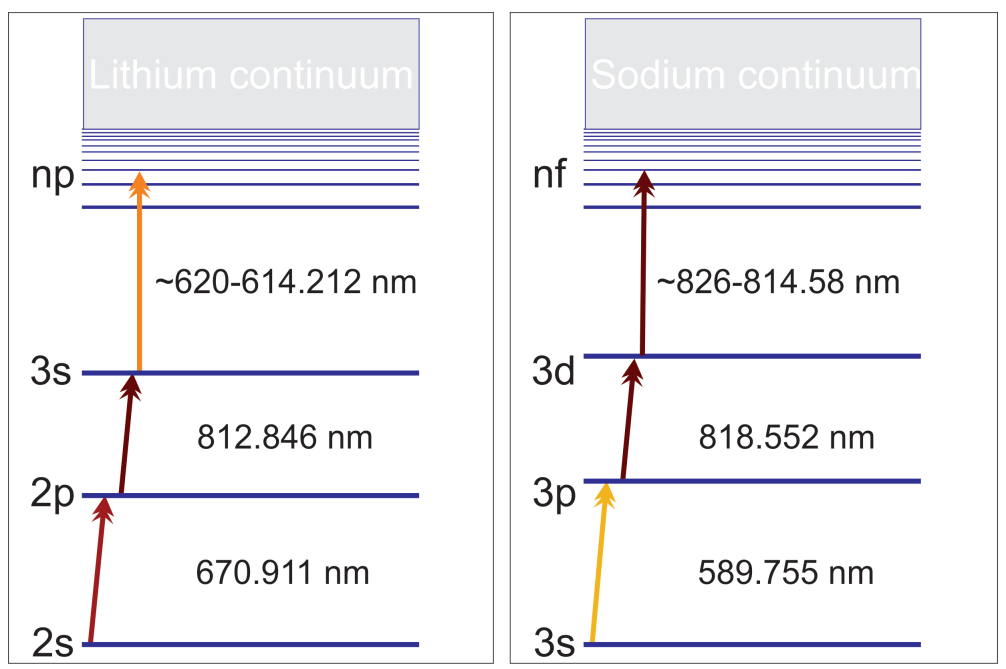

Figure 2.3: Energy level diagram to excite Li $n p$ and $\mathrm{Na} n f$ Rydberg states.

The 45-mW output of the laser, after passing through two isolators, is sent into a Pockels cell. To avoid undesirable photoionization of atoms in the $3 d$ state due to an absorption of extra photons from the first laser, it is triggered in such a way that, after passing through a polarizing beam splitter cube, the $\mathrm{cw}$ beam is off during the first dye laser pulse, but is on right before the second one.

\subsubsection{Dye Lasers}

To drive $2 s-2 p, 2 p-3 s$, and all $\mathrm{Na}$ transitions, Littman-Metcalf type dye lasers are used. With a cylindrical lens, the pump beam is focused just inside a glass dye cell through which a particular solvent with a dissolved dye constantly flows. The position and the intensity of the pump beam spot inside the dye should be controlled in order not to burn the dye (there should not be any sounds). The laser cavity is created by two mirrors on both sides of the dye cell and a diffraction grating between them as shown in Fig. 2.4. The end mirror reflects fluorescence towards the diffraction grating through the dye cell, and the tuning feedback mirror is set up to reflect back the first reflection order of the grating. If the position between the mirrors is correct, the dye spontaneous emission is amplified, and the cavity lases. The output of the cavity is taken from the zeroth order reflection of the diffraction grating. The tuning mirror is set on a rotating mount, so the 


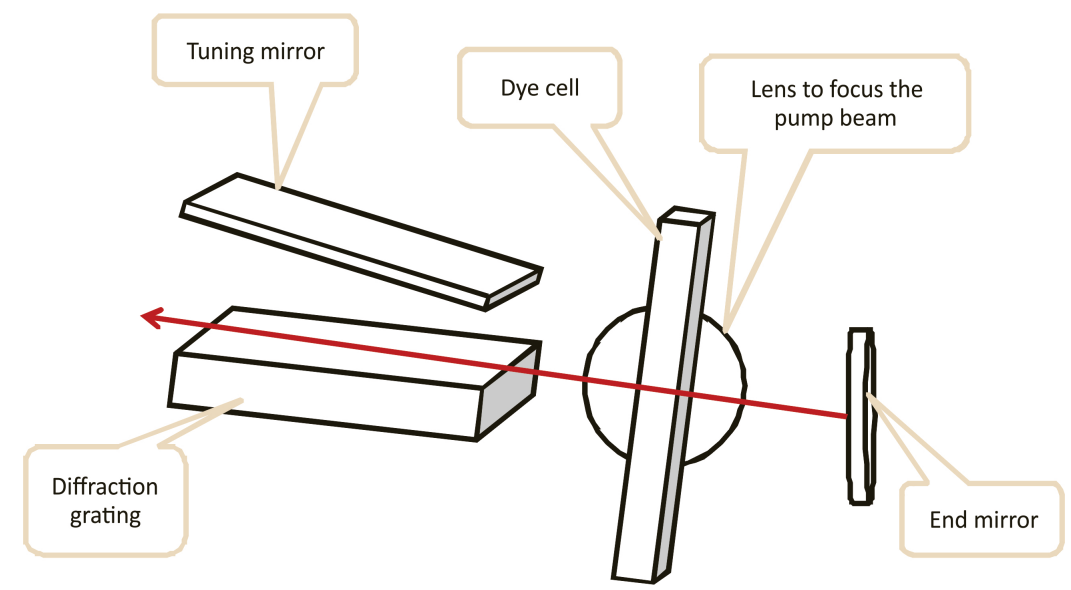

Figure 2.4: Schematic diagram of a Littman-Metcalf type dye laser provided by S. Niyaz. A cylindrical lens focuses the pump beam inside the dye cell, and a fluorescent dye flowing trough it serves as an active medium for the laser cavity that consists of two mirrors. The diffraction grating ensures relatively narrow linewidth of the laser output.

resonant frequency of the laser can be continuously tuned either by hand or via a computer controlled stepper motor. It is certainly advisable to set up such dye lasers perpendicular to the plane of polarization. For instance, the laser output is significantly improved when the diffraction grating is set up in a horizontal plane parallel to the incoming beam with the lines perpendicular to the laser polarization. An example of the $2 p-3 s$ IR dye laser is shown on a photograph in Fig. 2.5. Such set up is harder to built, but was shown to offer a significant improvement, and I mounted all of the dye lasers in this fashion.

The diffraction grating ensures relatively narrow linewidth of the laser that depends on how many lines are within the spot on the grating. The best linewidth is achieved when the grating is set up at a grazing incidence, which also results in a lower power of the laser output. As we do not require very narrow linewidth of the lasers making first atomic transitions, the grating is usually setup at a $\sim 30$ degree angle to the incoming beam. That results in a linewidth of $\sim 20-25 \mathrm{GHz}$, but significantly more power per $\mathrm{GHz}$ of the linewidth. The last transition lasers, on the other hand, have to be narrow so that higher Rydberg states can be resolved. In this case, the grating is set up at a grazing incidence. To further decrease the linewidth, I used a Littman double grating dye laser for the $3 s-n p$ transition in Li. In this laser, a tuning mirror is replaced with another diffraction grating. We could not use the double grating arrangement for the Na $3 d-n f$ laser, as a dye used for 


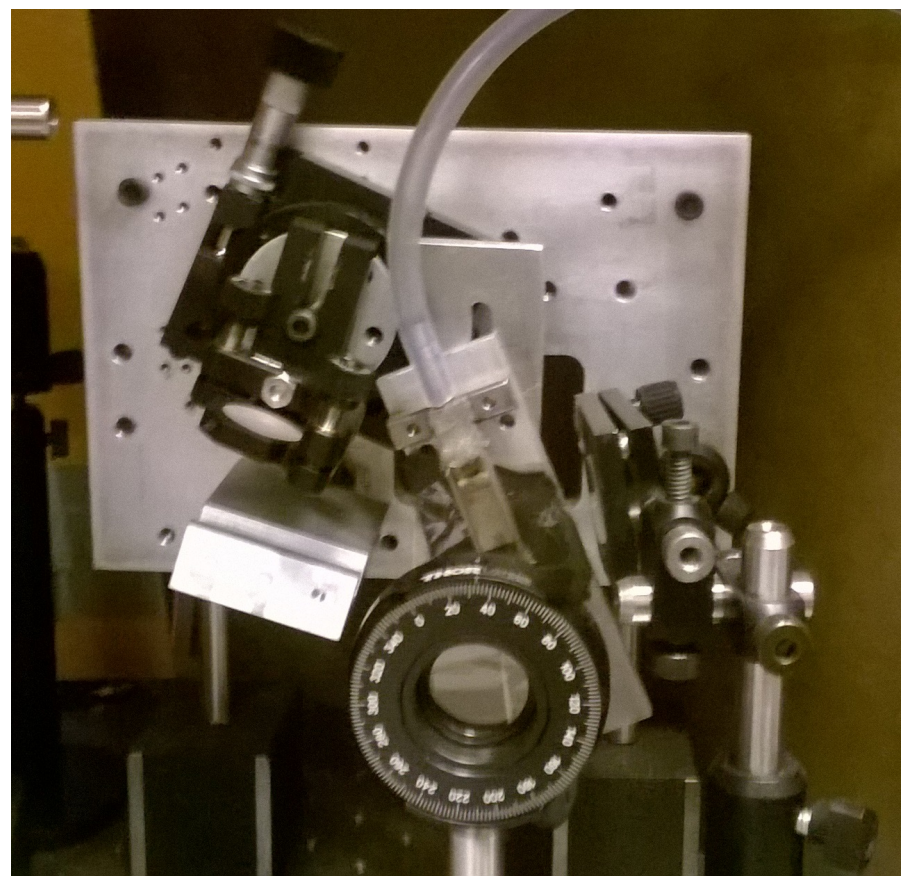

Figure 2.5: A photograph of an IR Littman-Metcalf type dye laser that I used to drive the $2 p-3 s$ transition in $\mathrm{Li}$. The tuning mirror is mounted to a precision rotating stage to ensure easy and fast tuning of the laser frequency.

this laser fluoresces weakly, and the output power was too low. Typically, I used diffraction gratings with 1200 lines/mm for IR lasers, and 1800 lines/mm for the ones lasing in red and orange spectra.

Picking the right dye, solvent, and concentration is a crucial step to ensure that the laser behaves as desired. Exciton offers a wavelength chart that helps to pick the right dye, but I suggest to use it as a guideline and experiment with different dyes and solvents. For Li $2 s-2 p$ and Na $3 s-3 p$ transitions, I used LDS-680 (70mg/0.5L) and Rhodamine$590(75 \mathrm{mg} / 0.5 \mathrm{~L})$ dyes dissolved in ethanol. For the last transition in $\mathrm{Li}, 3 s-n p$, I used Rhodamine-640 $(\sim 80 \mathrm{mg} / 0.5 \mathrm{~L})$ mixed with DCM $(\sim 30 \mathrm{mg} / 0.5 \mathrm{~L})$ dissolved in methanol. The problem with this laser was that its dye spontaneous emission was at $\sim 610.5 \mathrm{~nm}$ which matched a two photon $3 p \rightarrow 3 d \rightarrow$ continuum transition and resulted in a huge amount of ions present during the experiment. Hence, a requirement on that laser was to decrease 610-nm fluorescence power without lowering the power of the laser beam. For that reason, we also replaced the end mirror with a wedge mirror, and the output of the laser was taken 
from it and not from the diffraction grating.

To drive Li $2 p-3 s$, Na $3 p-3 d$ and $3 d-n f$ transitions an infrared dye is required. For previous experiments in Li, an LDS-821 dye dissolved in methanol was used for the $2 p-3 s$ transition. The output was very weak and unstable and required constant maintenance slowing the experiment. A big improvement was to change the solvent to ethanol and the concentration to $\sim 100 \mathrm{mg} / 0.5 \mathrm{~L}$, as well as carefully aligning the laser. The power of the laser was increased by more than a factor of 50 , and its output became much more stable without causing trouble any longer. It is worth noting that submerging the dye container into an iced water and cooling it down improves the output of the laser. Upgrading this laser, allowed us to use a novel excitation scheme for $\mathrm{Na}$ atoms driving the $3 d-n f$ transition with a tunable infrared Littman-Metcalf type dye laser. Another upgrade performed on the dye lasers was a modification of dye cells in order to narrow the space between the front glass and back metal surfaces of a cell. That increases the speed of the dye flow in the region where the pump beam is focused and improves the output power of the laser.

To excite atoms, the three laser beams are sent to the vacuum chamber and focused to less than 1-mm diameter spots where they cross each other and the atomic beam. The first two lasers (driving the first two transitions) are sent perpendicular to the atomic beam, nearly parallel to each other. The last transition laser counter propagates to the atomic beam creating a sphere-like interaction region. The laser fields are polarized vertically throughout the experiment unless stated otherwise. In some cases, however, half-wave plates are used to make the laser polarizations perpendicular to the microwave field polarization and excite $|m|=2$ states.

\subsection{Microwave System}

A general goal of the experiment we were conducting was to study an interaction of Rydberg atoms with microwave fields. Hence, it is required to produce microwave field pulses with a well-defined frequency, amplitude, width, and polarization. We conducted three distinct experiments that required microwave fields with 17,38 , and $80-\mathrm{GHz}$ frequen- 
cies. In all cases, we used microwave cavities inside the vacuum chamber that had to be built and mounted differently. Many microwave components to generate microwave pulses, on the other hand, were shared.

\subsubsection{Microwave Components}

Microwave pulses in the range 10-20 GHz are generated by a Hewlett Packard (HP) 83550B sweep oscillator with a 83550A RF plug-in. Its output is modulated internally to a pulsed mode by a 8112 HP pulse generator. The resulting pulse has a variable width and rise and fall times of approximately $15 \mathrm{~ns}$. Going through an HP 8494B attenuator, it is amplified in a Hughes $8020 \mathrm{H}$ traveling wave tube amplifier if frequencies below $20 \mathrm{GHz}$ are required. It is then sent through a HP P375A variable attenuator into the vacuum chamber using SMA cables and into the cavity through an iris in one of the cavity mirrors.

To produce the 38-GHz microwave field, the 19-GHz pulsed output of the sweep oscillator is frequency doubled by a Phase One SP40 frequency doubler, sent through an isolator and a 0- to 50-db variable HP R382A attenuator into the vacuum chamber using WR-28 waveguide and into the cavity through an iris in one of the cavity mirrors. In order to monitor the pulse, a 20-db directional coupler is used, and a crystal detector is set to detect the power reflected from the microwave mirror.

For the $80-\mathrm{GHz}$ experiment, the sweep oscillator output goes through two frequency doubling stages. First, the frequency is doubled to $38.5 \mathrm{GHz}$ by a Phase One SP40 doubler with an isolator on the output, amplified by a Microwave Power L3640-37 amplifier, and sent through a 0- to 50-db variable HP R382A attenuator into the vacuum chamber using a WR-28 waveguide. Inside the vacuum chamber, the microwave pulse is frequency doubled again by a passive Virginia Diodes D80R7 doubler and is fed into the microwave cavity by a WR-12 waveguide through an iris in a cavity mirror. To monitor the microwave pulse and the reflected power, two $80-\mathrm{GHz}$ crystal detectors are used. One is connected to a 10$\mathrm{db}$ directional coupler inside the vacuum chamber before the cavity to watch the reflected power, while the other one is connected to the output mirror of the cavity to detect the leaking power out of the cavity. Both crystal detectors are connected to an oscilloscope via 
sma feed-throughs in one of the chamber flanges.

\subsubsection{Microwave Cavities}

A Fabry-Perot microwave cavity, typically, consists of two large brass mirrors with small irises in the center. An example of such a cavity is shown in Fig. 2.6. While difficult to set up and maintain, such microwave cavities offer certain advantages. Just like in an optical cavity, a standing wave is created between the mirrors, and the cavity can operate only on cavity modes set by the mirror separation, additionally serving as a filter for other frequency components present in the incoming beam. Also, the microwave field amplitude is approximately Q times stronger than the field after a horn and is well-defined. Moreover, atoms in a small interaction region experience nearly uniform electric field, as opposed to high-intensity laser experiments where the gradient across the beam profile has to be taken into account.

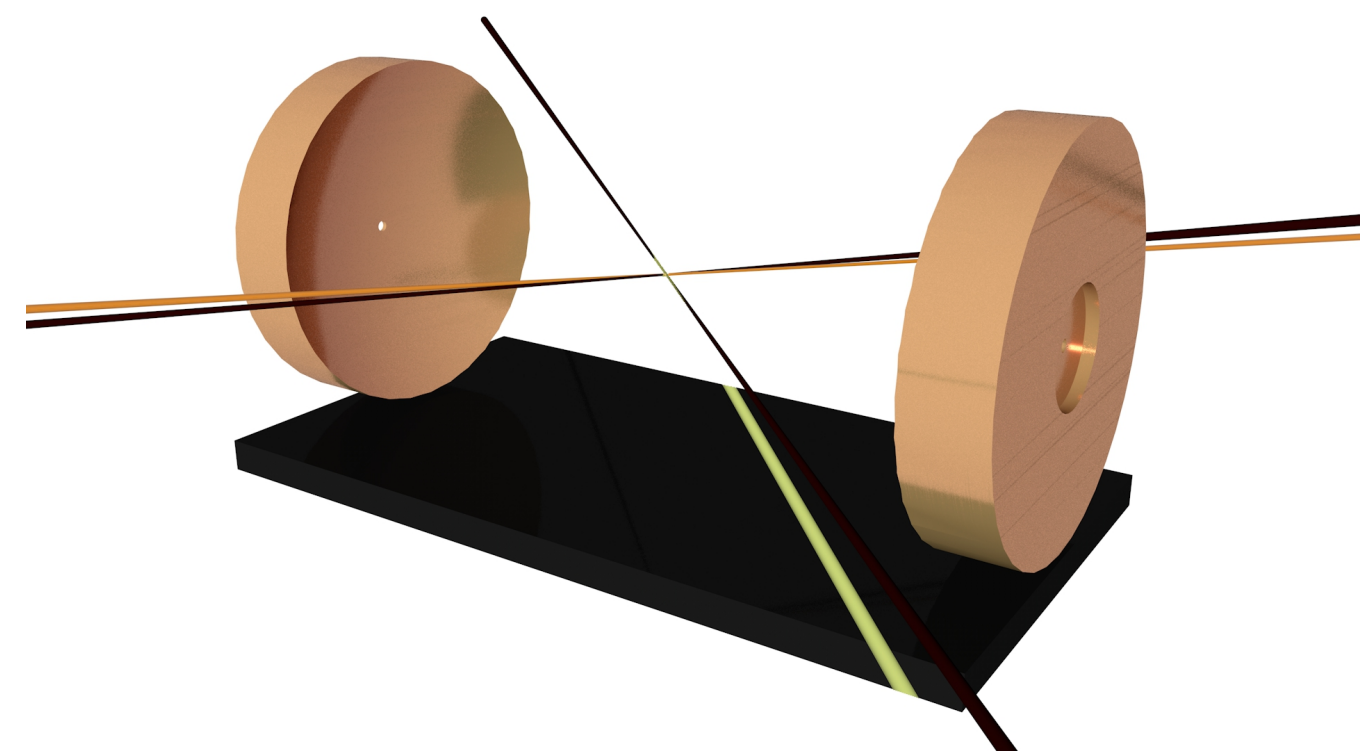

Figure 2.6: Two brass mirrors shown constitute an 80-GHz microwave cavity. Laser beams cross each other and the atomic beam at the center of the cavity. The mirrors are $69.7 \mathrm{~mm}$ in diameter with $52-\mathrm{mm}$ radii of curvature.

As was mentioned before, experiments at 17,38 , or $80 \mathrm{GHz}$ require different cavity mirrors and different constructional solutions. Parameters of the microwave cavities built 
for these experiments are listed below. The first Fabry-Perot microwave cavity consists of two brass mirrors $102 \mathrm{~mm}$ in diameter with 119-mm radii of curvature. The on-axis spacing between mirrors is $101.5 \mathrm{~mm}$. The cavity is operated on the $\mathrm{TEM}_{012}$ mode at a frequency of $16.91 \mathrm{GHz}$ with $Q=3200$. The filling time of the cavity, $\tau=Q / w=29 \mathrm{~ns}$, sets the lower limit limit on the pulse width. The second microwave cavity has mirrors 40.5 $\mathrm{mm}$ in diameter with $75.9-\mathrm{mm}$ radii of curvature. The on-axis spacing between mirrors is $44.5 \mathrm{~mm}$. The cavity is operated on the $\mathrm{TEM}_{012}$ mode at a frequency of $38.34 \mathrm{GHz}$ with $Q=5100(\tau=21 \mathrm{~ns})$. Mirrors of these cavities had irises large enough to allow the first and the second dye laser beams to go through without scattering, so the cavities were set up perpendicular to the atomic beam and the last laser beam.

The last Fabry-Perot microwave cavity consists of two brass mirrors $69.7 \mathrm{~mm}$ in diameter with $52-\mathrm{mm}$ radii of curvature. The on-axis spacing between mirrors is $90.51 \mathrm{~mm}$. The cavity is operated on the $\mathrm{TEM}_{048}$ mode at a frequency of $79.05 \mathrm{GHz}$ with $Q=8600(\tau=17$ ns). Here irises had to be made small, and the cavity was set 45 degrees to the atomic or laser beams. In all cases, cavities are mounted in such a way that the center of the cavity is vertically aligned with the center of the oven, and laser beams cross the atomic beam at an antinode of the microwave field. A lens focusing the last dye laser beam can be displaced horizontally with a $0.1-\mathrm{mm}$ precision ensuring the excitation of atoms occurs at a maximum of the microwave field that can be checked experimentally.

How the microwave field amplitude can be calculated is discussed in detail in J. Gurian's thesis [47] and more extensively in [49], so I omit the derivation and present a formula that was used to calculate field amplitudes of each cavity:

$$
E=\sqrt{\frac{4 Q P_{i n}}{\pi^{2} \nu \epsilon_{0} \omega_{0}^{2} l}},
$$

where $l$ is the spacing between mirrors, $\omega_{0}$ is the beam waist radius (the beam is assumed to be gaussian), $\nu$ is the frequency, and $P_{i n}$ is the input power accounting for the coupling loss. The microwave systems that were used in the experiments generated a $16.91-\mathrm{GHz}$ pulse with 0 - to $300-\mathrm{V} / \mathrm{cm}$ amplitude, a $38.34-\mathrm{GHz}$ pulse with 0 - to $90-\mathrm{V} / \mathrm{cm}$ amplitude, 
and a 79.05- $\mathrm{GHz}$ pulse with 0 - to $180-\mathrm{V} / \mathrm{cm}$ amplitude. In all cases it was possible to vary the width of the pulse from a minimum filling time of the cavity to several microseconds. The microwave field amplitude was calibrated using the method described by Cheng et al. [50]. The directional couplers set to monitor the reflected power from the microwave mirror allow measuring the coupling loses to the cavity. In all cases, we measured the microwave power coming out of the waveguide before the mirror, and the consistency of the power level was checked on a daily basis with crystal detectors. We are able to determine the amplitude of the pulse with an uncertainty of $10-15 \%$.

\subsection{Detection and Data Acquisition Methods}

The apparatus is triggered at a 1-kHz repetition rate from the output of the Q-switched Nd:YLF laser. The experimental timing is controlled by an SRS DG535 Four Channel delay generator that triggers the Pockels cells, the microwave sweep oscillator, the field ionization pulse trigger generator, oscilloscopes, and the integrating boxcars.

\subsubsection{Field Ionization Pulse}

Excited Rydberg atoms that survive the microwave field have to be field ionized in order to be detected. It is possible to ionize them with a static field ionization pulse (FIP) if its amplitude exceeds the ionization threshold of the atoms in question. The FIP is applied to the bottom plate, which is shown in Fig. 2.7, usually about $300 \mathrm{~ns}$ after the end of the microwave pulse. The FIP not only ionizes atoms, but also pushes the charged particles created in ionization, electrons or ions, depending on the polarity of the pulse, towards the microchannel plate detector $(\mathrm{MCP})$ through the hole in the plate above the interaction region. It is well-known that Rydberg atom static field ionization scales as $n^{-4}$ [1]. Thus, a slowly-rising field ionization pulse can be utilized to separate signals from Rydberg states with different $n$.

Fig. 2.8 shows a typical circuit diagram based on an SCR transistor, and a resulting FIP is presented in an oscilloscope trace in Fig. 2.9. It has a $1.3-\mathrm{kV}$ amplitude and 1.16- $\mu$ s 


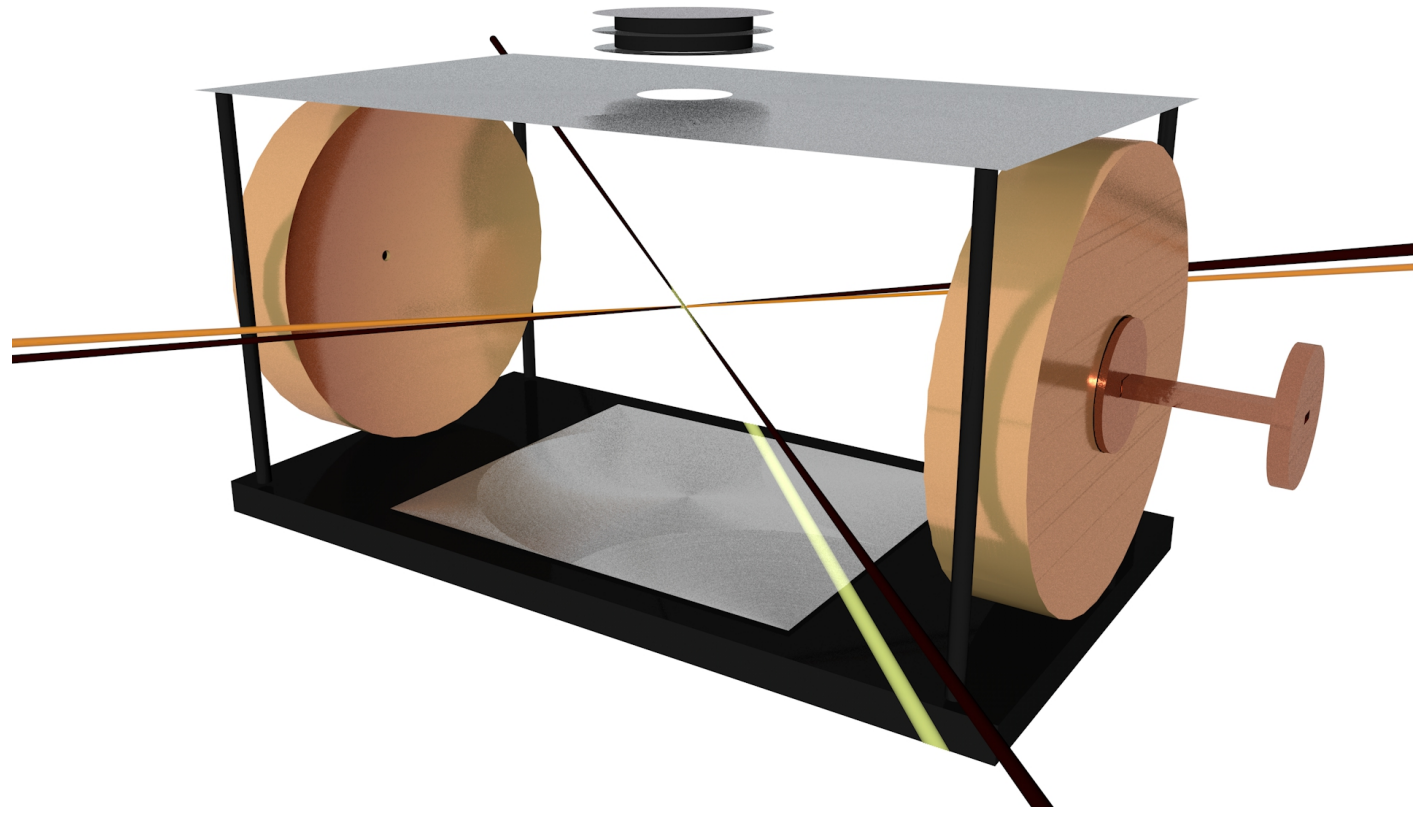

Figure 2.7: Laser beams cross each other and the atomic beam to form an interaction region at an antinode of the microwave electric field at the center of the $80-\mathrm{GHz}$ cavity. The FIP is applied to the bottom plate, and charged particles are pushed towards the MCP detector through a hole in the plate above the interaction region. The microwave field is fed through a small iris in one of the mirrors with a WR-12 waveguide connected to it.

rise time. In different experiments various Rydberg states had to be ionized. When working with mid-range Rydberg states $(n>50) \sim 3-\mathrm{kV}$ amplitude FIP is sufficient. Parameters of the components required to generate such a pulse are shown in the top right corner of Fig. 2.8. However, when lower Rydberg states need to be ionized, a larger FIP is necessary. A different FIP circuit was built by my labmate Kapila Wijayaratne that required a highcurrent power supply and high-power components that are listed in the bottom right corner of Fig. 2.8. It is capable of generating a $8.5-\mathrm{kV}$ pulse, but had to be extensively cooled down by two AC fans due to a heat dissipation in the resisters.

While using a large FIP to study low Rydberg states is great, it usually prevents detection of highly excited states. Some portion of the FIP is picked up by the microwave mirrors and side plates surrounding the cavity, so low-energy electrons created in the very beginning of the FIP can be deflected sideways and do not reach the MCP. For that reason, in some cases when the high-lying states were studied, a Tektronix AFG3011 arbitrary waveform 


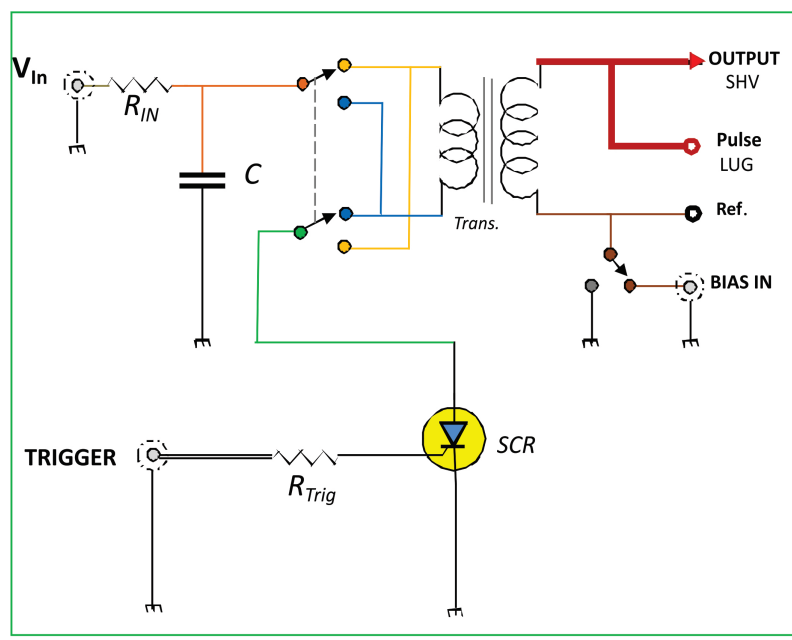

\begin{tabular}{|c|c|c|}
\hline SCR & MCR 265 & $\begin{array}{l}108 \mathrm{~V} \\
\text { Std. level }\end{array}$ \\
\hline Trans. & T 105 B & $1: 30$ \\
\hline C & $900 \sim \mathrm{nF}$ & $\begin{array}{l}600 \mathrm{~V} \\
\text { Mylar }\end{array}$ \\
\hline$R_{\text {in }}$ & 5 KO & $9 w$ \\
\hline $\mathrm{R}_{\text {Trig }}$ & 115 KO & $1 / 4 W$ \\
\hline SCR & MCR 265 & $\begin{array}{l}108 \mathrm{~V} \\
\text { Std. level }\end{array}$ \\
\hline Trans. & T 105 B & $1: 30$ \\
\hline C & $470^{\sim} \mathrm{nF}$ & $\begin{array}{l}600 \mathrm{~V} \\
\text { Mylar }\end{array}$ \\
\hline $\mathrm{R}_{\mathrm{in}}$ & 1 ко & $200 \mathrm{~W}$ \\
\hline $\mathrm{R}_{\text {Trig }}$ & 115 KO & $1 / 4 W$ \\
\hline
\end{tabular}

Figure 2.8: A circuit diagram to generate a FIP provided by K. Wijayaratne. Two sets of values are shown. A circuit with the top set components generates a regular FIP with a $3-\mathrm{kV}$ output at $100-\mathrm{V}$ input at $1 \mathrm{kHz}$ repetition rate. The other set includes high-power resistor and capacitor to generate 8-kV FIP at $300-\mathrm{V}$ input at the same repetition rate. This circuit is very large as it includes two big AC fans for cooling purposes.

generator was used to produce a 4-V square pulse that ionized only extremely high-lying states but did not introduce any detection efficiency problems.

It is possible to detect ions or electrons by switching the polarity of the FIP. A negative (positive) input voltage pulse leads to what we term a negative (positive) FIP and the detection of electrons (ions). The 300-ns delay between the microwave pulse and the FIP provides enough time for the electrons produced by photoionization or microwave ionization to leave the interaction region before the start of the FIP. Thus, with a negative FIP, only atoms which are left in bound states after the microwave pulse are detected. Since the flight time of the electrons from the interaction region to the MCP is negligible compared to the rise time of the FIP, the time resolved MCP signal yields the final bound state distribution subsequent to the microwave pulse.

With a 300-ns delay and a positive FIP we detect all ions produced; those due to photoionization, microwave ionization, and field ionization. For example, with a $\sim 75-\mathrm{V} / \mathrm{cm}$ FIP, we can ionize states as low as $n=60$, and with a relatively small FIP, $\sim 10-\mathrm{V} / \mathrm{cm}$, we detect ions created by the microwave pulse, photoionization, and field ionization of only higher lying states. Due to the fact that ions do not leave the interaction region in 300 


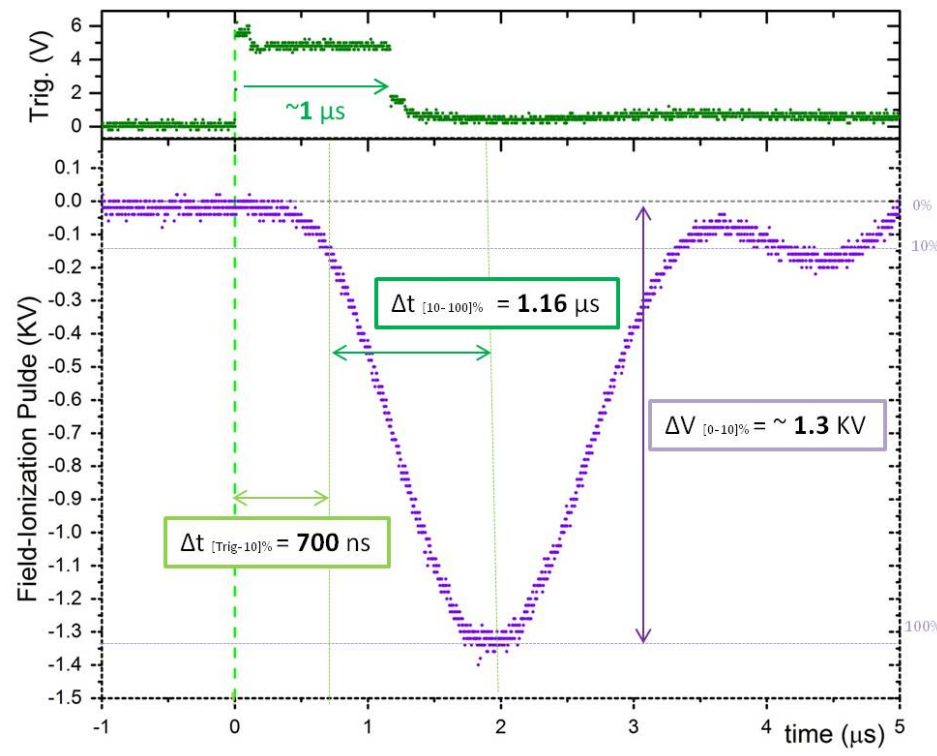

Figure 2.9: An oscilloscope trace showing the FIP, taken with the help of a high-voltage probe, and the trigger pulse. The FIP has $1.3-\mathrm{kV}$ amplitude and $1.16-\mu s$ rise time at $30-\mathrm{V}$ input.

ns and their long flight times to the MCP, it is impossible to distinguish a population left in the extremely high-lying states from ions created by microwave ionization of the initial Rydberg state (Laser photoionization is negligible.). It is, however, possible to discriminate between these ion signals and field ionization of atoms in states of $n \leq 90$.

\subsubsection{Microchannel Plate Detector}

Charged particles created in ionization of Rydberg atoms are detected in a dual microchannel plate detector. Microchannel plates are separated by metal disks, as shown in Fig. 2.10, and the high-voltage is applied between them. The circuit shown in Fig. 2.10 is, in fact, outside the vacuum chamber which allows quick adjustments without the need to open it. The principle of operation of the MCP is the following: a charged particle hits a channel on the front plate, and an electron avalanche is created with the gain around $10^{4}$. The second plate further increases the number of created electrons that are collected in an anode behind it. The MCP assembly is mounted above the hole in the top plate, as shown in Fig. 2.7, and can detect electrons or ions depending on the polarity of the potential 


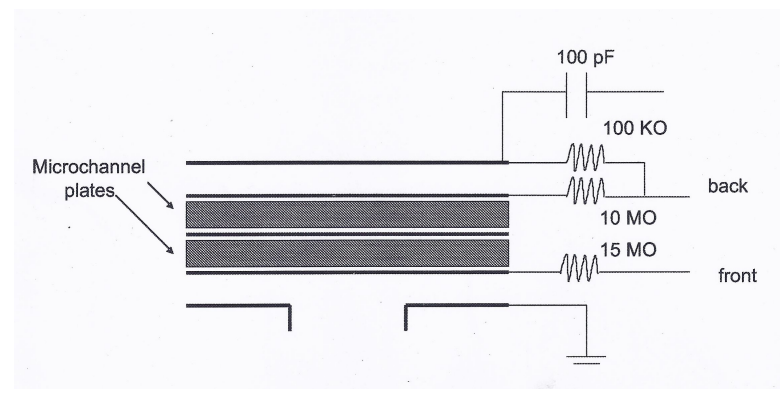

Figure 2.10: The MCP circuit and connection diagram.

across its plates. Usually, I operated the MCP with the 1.8-2.3-kV input voltage.

\subsubsection{Data Acquisition}

The MCP signal is amplified in a SRS SR445A amplifier and is either captured in a SRS SR250 gated integrator/boxcar averager or recorded as an oscilloscope trace. We can choose when to put the integration gate of the boxcar, recording either the signal from the states with selected binding energies or all bound states that can be ionized by the FIP. When the boxcar is used, the signal going to the boxcar is averaged over 300 samples and sent to the computer for recording. Since higher lying states are ionized earlier in the rising FIP, the time resolved oscilloscope traces can be used to infer the final state distribution of bound states surviving the microwave pulse. As will be discussed below, a measurement essential for the described experiment is a continuous frequency scan of the laser that makes the Rydberg transition while recording the MCP signal at the same time. Labview computer programs that I wrote are capable of capturing oscilloscope traces or averaged data points from the boxcar in real-time, simultaneously applying the voltage to a stepper motor that scans the laser frequency. In order to measure the laser frequency, we can record a signal from the Coherent WaveMaster wavemeter through an RS232 connector. Before implementing that, a relative frequency measurement of this laser was obtained with the help of a 52.42-GHz free spectral range etalon, and an optogalvanic signal from the 16274.0212-cm ${ }^{-1} 2 \mathrm{p}^{5}\left(2 \mathrm{p}_{3 / 2}\right) 3 \mathrm{~s}-2 \mathrm{p}^{5}\left(2 \mathrm{p}_{3 / 2}\right) 2 \mathrm{p}$ Ne line provided an absolute calibration. Both etalon and optogalvanic signals were recorded in a computer after averaging in boxcars. 


\subsection{Canceling Static Stray Fields}

The biggest experimental challenge of this work turned out to be nulling the static fields in the interaction region. One of the main goals of the experiment was to study the extremely high-lying states that are readily ionized by very small static fields. Moreover, our results showed that even processes like microwave ionization of low Rydberg states depend significantly on the static field distribution. Hence, it is crucial to be able to control and null static fields present during the experiment. There are three major sources of non-zero static field: free charged particles in the interaction region, surface fields on the components surrounding the interaction region, rf noises from electronics in the lab.

In order to remove charged particles, the oven has be biased with a voltage, about $1 \mathrm{~V}$, large enough to deflect free charges from the beam. Also, it is very important to eliminate any photoionization of atoms that forestalls excitation to a Rydberg state. To decrease an impact of surface patch fields, it is recommended to remove all the surfaces as far from the interaction region as possible. Canceling completely the rf noises is the most difficult task. The largest contribution to it comes from the Pockels cells high-voltage pulses that nearly coincide with the excitation timing. Low pass filters were placed on all metal plates around the interaction region, but the one for the FIP, decreasing the effect of the rf noise picked up by the plates or cavity mirrors.

To null and control the static field, the interaction region is surrounded by metal plates from all sides, as shown in Fig. 2.11, each of which, including the cavity mirrors, can be separately biased by power supplies outside the chamber. By iteratively scanning the potential on each plate during the experiment, as will be discussed below, it is possible to find a set of voltages that corresponds to the smallest achievable static field. While conducting this experiment, I was able to lower the static field to $2 \mathrm{mV} / \mathrm{cm}$.

\subsection{Rydberg Spectrum}

To familiarize the reader with a typical measurement that is acquired with the help of the described above experimental apparatus, a spectrum of Rydberg states of lithium 


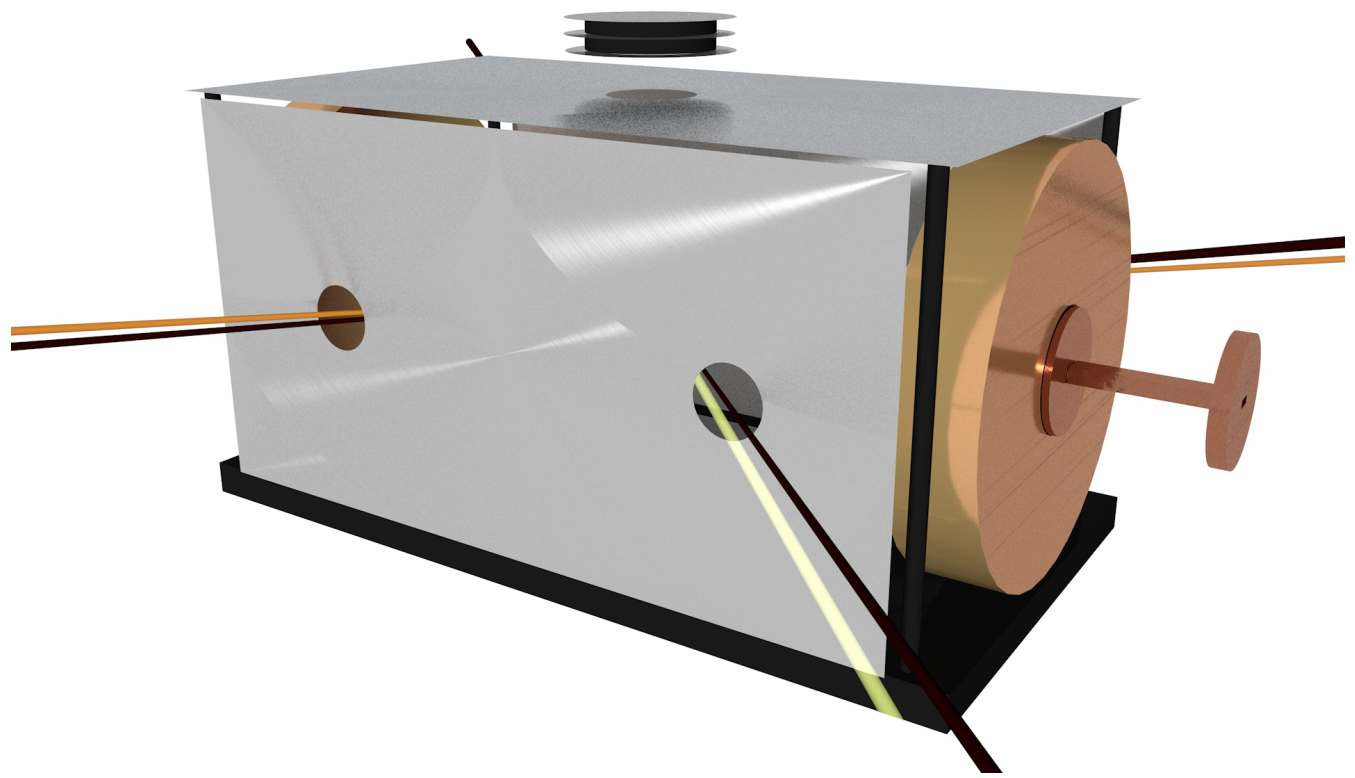

Figure 2.11: The interaction region is surrounded by metal plates and cavity mirrors from all sides, each of which can be separately biased to control the static field distribution. The $80-\mathrm{GHz}$ cavity is shown that had to be mounted at a 45 degree angle to the atomic beam propagation. Laser and atomic beams reach the interaction region through the holes drilled in the side plates. In case of the $80-\mathrm{GHz}$ cavity, the spacing between brass mirrors is about $10 \mathrm{~cm}$, side plates $-8 \mathrm{~cm}$, bottom ant top plates $-10 \mathrm{~cm}$.

is shown in Fig. 2.12. Atoms are excited to a Rydberg state, and 300 ns later, bound atoms are ionized by a negative FIP. Electrons are detected, and a survival probability is measured. The amplified MCP signal is averaged in the boxcar with the integration gate set to detect signal from all bound states. The frequency of the last transition laser is scanned continuously by a computer controlled stepper motor that rotates a tuning mirror of the dye laser. For each step of the motor, the computer records a data point from the boxcar that averages the MCP (field ionization) signal as well as a data point from the wavemeter or etalon and optogalvanic signals. From such a data set, a spectrum of Rydberg states can be reconstructed, like the one shown in Fig. 2.12.

For convenience, the horizontal axis is calibrated to measure the laser frequency from the zero-field ionization limit that is denoted as zero on the graph. Negative energies, given here as frequencies, correspond to bound states, while positive energies are in the continuum. To obtain the spectrum of Fig. 2.12, we tuned the laser to $-1100 \mathrm{GHz}$ below the limit and scanned it towards positive energies, and the first peak corresponds to a 


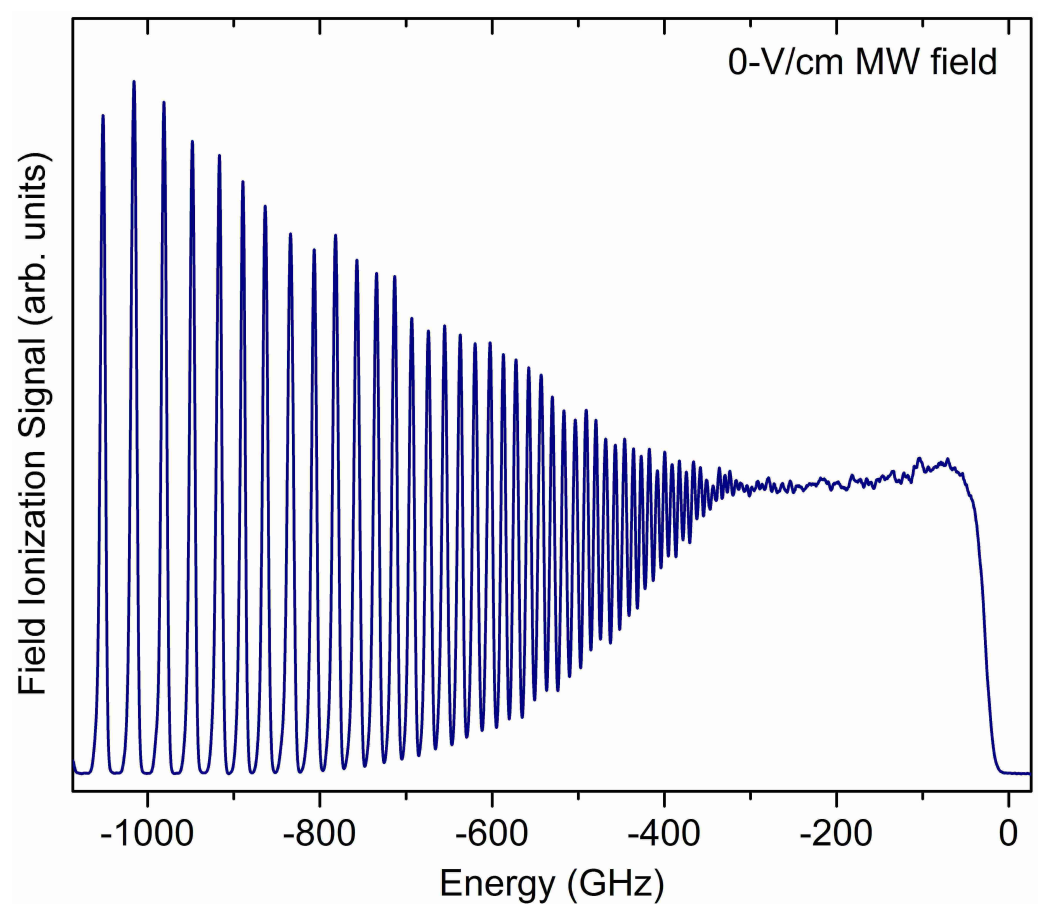

Figure 2.12: Field ionization signal as a function of the laser frequency tuning, in terms of energy relative to the ionization limit (IL). The horizontal axis is calibrated by the etalon and optogolvanic signals. The first peak on the graph corresponds to $n=56$, at $-1056 \mathrm{GHz}$. Atoms are excited in the presence of a $18.5-\mathrm{mV} / \mathrm{cm}$ static field that depresses the IL by $25 \mathrm{GHz}$. Signal above the IL (0 on the x-axis) is zero, as the electrons created in laser photoionization leave the interaction region before the onset of the FIP and are not detected. In this case a survival probability is measured.

Rydberg states with $n=56$. As the laser frequency is changed, more peaks are observed that belong to higher Rydberg states. Around $-800 \mathrm{GHz}$, peaks are not fully resolved, and about $-400 \mathrm{GHz}(\sim n=100)$ the signal becomes flat, as the separation between Rydberg states is smaller than the linewidth of the laser. At an energy of $-45 \mathrm{GHz}$, the signal starts falling to zero because photoelectrons are not detected when the laser is tuned over the ionization limit. Due to the finite linewidth of the laser, the signal drops to zero with a finite slope, and we denote the middle of that slope as the depressed ionization limit. The limit is depressed due to a nonzero static field in the interaction region. We estimate that stray field using $\Delta W=2 \sqrt{E}$ (Eq. 1.6). In this case, the ionization limit is depressed by about $25 \mathrm{GHz}$ which corresponds to $18.5-\mathrm{mV} / \mathrm{cm}$ static field. In order to cancel the static field, it is required to tune the laser to the center of the slope and change the bias voltages on plates surrounding the interaction region until the MCP signal, seen on the oscilloscope, is maximized, and the limit shifts towards positive energies. Scanning the laser across the 
limit and extracting its shift from zero provides us with a way to measure small static fields that are present during the excitation. It is also very important to check that the MCP signal actually drops to zero when the laser is tuned above the ionization limit. When the static field is close to zero and the number of ions in the interaction region is substantial, free electrons can be trapped in the ion cloud forming low-energy plasma. I observed such plasma on several occasions which manifested itself as the non-zero signal extending over the ionization limit. In most cases, lowering the laser intensity removes the plasma. It is necessary to perform laser scans across the ionization limit with the microwave field off on a daily basis before starting the experiment to ensure that the static field amplitude is as desired and no electrons can be trapped in the plasma. 


\section{Chapter 3}

\section{Ionization of Rydberg atoms by 17 , 38 , and $80-\mathrm{GHz}$ microwave fields}

\subsection{Introduction}

In photoionization there is a frequency requirement for the onset of ionization, in field ionization there is a field requirement, and midway between these two processes lies multiphoton ionization, which has both requirements. Microwave ionization appears to be a way to systematically connect these apparently different phenomena. Specifically, it is possible to create high-lying Rydberg states, which may be ionized by the absorption of a single microwave photon, as well as low-lying states in which the variation in the microwave field is slow enough compared to the motion of the Rydberg electron to be quasi-static. Thus, by fixing the microwave frequency and changing the principal quantum number $n$ of the atom it is possible to pass from photoionization to field ionization.

The first effort in this direction was the work of Bayfield and Koch [15], who studied the ionization of $\mathrm{H}$ atoms of $n \sim 65$ by fields of frequencies $9.9 \mathrm{GHz}, 1.5 \mathrm{GHz}$, and $30 \mathrm{MHz}$. Although presented in different terms, their results show that at the lower two frequencies $50 \%$ ionization occurs at the field $E=1 / 9 n^{4}$, the same value as the static field at which the extreme red Stark state ionizes. A somewhat lower field is required for ionization by a $9.9 \mathrm{GHz}$ field, a frequency approaching the Kepler, or $\Delta n=1$ frequency of $24 \mathrm{GHz}$. Later work confirmed that if the microwave frequency $\omega$ is well below the Kepler frequency, $\omega_{K}=1 / n^{3}$, ionization occurs from the initially populated $n$ state, at the field required for 
field ionization [51]. However, as the ratio of the microwave frequency $\omega$ to $\omega_{K}$ approaches one, multiphoton ionization, by transitions through either real or virtual higher lying levels, becomes possible, lowering the required field for ionization. While quantum calculations of the ionization fields in this regime have only recently become possible $[22,52]$, some time ago classical calculations, based on the onset of chaotic motion, provided an accurate description of ionization in the regime $\omega \cong \omega_{K}$ [53]. Although one might expect the classical model to work better for higher $n$ states, where $\Omega>1$, it predicts that at a fixed frequency the microwave field required for ionization decreases with $n$ [54]. However, experiments have shown that for $\Omega>1$ the ionization field only depends on the microwave frequency until the initial binding energy is within a few microwave photons of the ionization limit $[24,23]$. When the initial Rydberg state is within one or two microwave photons of the ionization limit, we would expect perturbation theory to give an accurate prediction of the ionization rate. However, in the experimentally accessible regime the ionization rates are at least five times lower than expected [23], although perturbation theory is undoubtedly applicable in an inaccessibly low microwave power regime.

In this chapter, we discuss ionization by microwave fields in the presence of small static fields. The results of three experiments are presented. The first experiment is concerned with ionization of Li $n p$ and Na nf Rydberg atoms by 17-GHz fields. The main goal of this experiment is to revisit ionization problems below $20 \mathrm{GHz}$ in order to show the significant impact of $1-\mathrm{V} / \mathrm{cm}$ static fields on ionization fields and state distribution after the microwave pulse. The other two experiments study ionization of Li $n p$ and $\mathrm{Na} n f$ states by 38 and 80-GHz fields, respectively, for a wide range of initial states. These novel investigations aim to provide an insight into an ionization regime different from the one observed at $17 \mathrm{GHz}$.

\subsection{Ionization of $\mathrm{Li}$ and $\mathrm{Na}$ Rydberg atoms by $17-\mathrm{GHz}$ mi- crowave fields}

Recent observations of the high-lying state production in $\mathrm{Li}$ after $38-\mathrm{GHz}$ microwave pulses, that will be discussed in Chapters 4 and 5, made us revisit ionization problems for 
fields below $20 \mathrm{GHz}$ in order to clarify if that phenomenon is peculiar to Li or high-frequency fields only. Moreover, previous experiments showed that $|m|=0$ and 1 Rydberg states of $\mathrm{Na}$ ionize at $15-\mathrm{GHz}$ by a sequence of transitions to higher states at a field $E=1 / 3 n^{5}$, while $|m|=2$ states ionize by field ionization at the field $1 / 9 n^{4}[13,14]$. It was observed that after the microwave pulse, atoms are either in the initial state or ionized: no population in the high-lying states was observed. In the following we report the ionization of $\mathrm{Li}$ and $\mathrm{Na}$ by a $16.9-\mathrm{GHz}$ microwave field to address the question of how static fields affected previous ionization experiments. As we shall see, the way the earlier experiments were conducted would have precluded leaving atoms in high-lying states or detecting them. We show that the Na microwave ionization field of $E=1 / 3 n^{5}$ is only valid for $|m|=0$. It is only observed for $|m|=1$ when there is a static field of $\sim 1 \mathrm{~V} / \mathrm{cm}$ present. In the sections which follow we outline the experimental technique, present our results, and discuss the conclusions which can be drawn from them.

\subsubsection{Experimental Approach}

A more detailed general explanation of the experimental setup is given in Chapter 2, and here we briefly describe specifics of the experimental apparatus used for this particular experiment. A thermal beam of ground state lithium or sodium atoms crosses the electric field antinode at the center of a 16.91-GHz Fabry-Perot microwave cavity. There atoms are excited to a Rydberg state by a sequence of transitions induced by 20 -ns dye laser pulses. Following laser excitation, atoms are subjected to a microwave pulse, typically 200-ns long, as shown in the timing diagram of Fig. 3.1. About $300 \mathrm{~ns}$ later atoms are exposed to a $1-\mu \mathrm{s}$ rise time field ionization pulse (FIP). Depending on the polarity of the FIP, either electrons or ions are sent upwards to a dual microchannel plate (MCP) detector. The MCP signal is amplified, captured by a gated integrator or oscilloscope, and recorded in a computer for later analysis.

The Fabry-Perot microwave cavity consists of two brass mirrors $102 \mathrm{~mm}$ in diameter with $119-\mathrm{mm}$ radii of curvature. The on-axis spacing between mirrors is $101.5 \mathrm{~mm}$. The cavity is operated on the $\mathrm{TEM}_{012}$ mode at a frequency of $16.91 \mathrm{GHz}$ with $Q=3200$. 


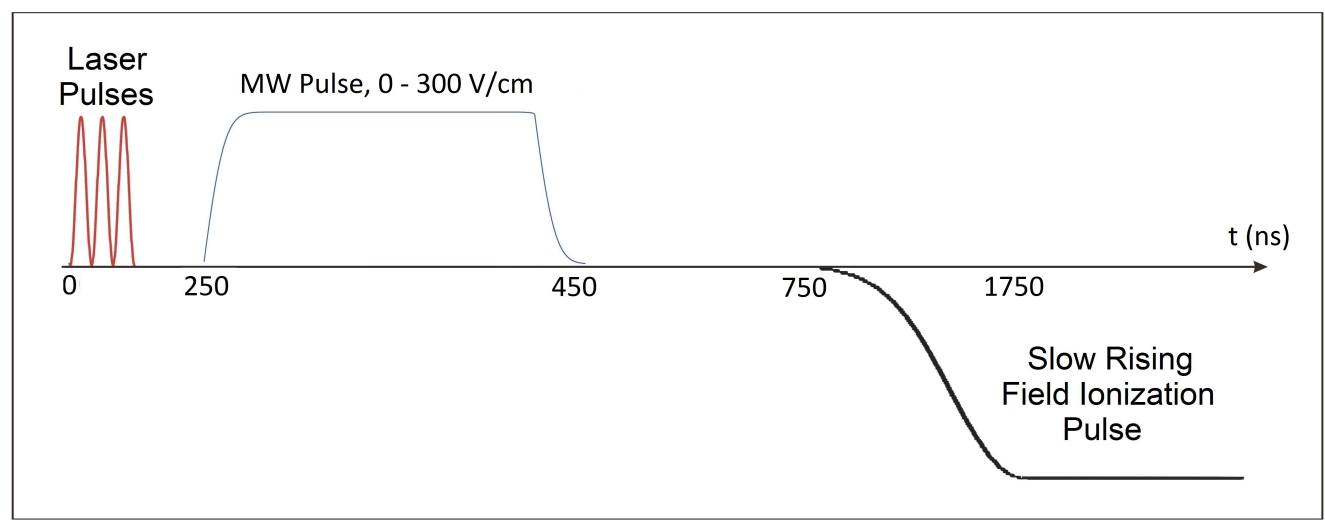

Figure 3.1: Experimental timing diagram. Rydberg states created in laser excitation are subjected to the microwave pulse. About 300 ns later a slowly rising FIP ionizes atoms, and either electrons or ions are detected by the MCP, depending on the polarity of the FIP.

The microwave system generates a $16.91-\mathrm{GHz}$ pulse with 0 - to $300 \mathrm{~V} / \mathrm{cm}$ amplitude and a variable width. The experiment is triggered at the $1-\mathrm{kHz}$ repetition rate of the Nd:YLF pump laser. Li atoms are excited via the $2 s \rightarrow 2 p \rightarrow 3 s \rightarrow n p$ transitions. The linewidth of the last laser is about $8 \mathrm{GHz}$, and its frequency can be continuously tuned to populate any Rydberg state of $n>20$. Na atoms are excited via $3 s \rightarrow 3 p \rightarrow 3 d \rightarrow n f$ transitions. The linewidth of the last laser is about $12 \mathrm{GHz}$. Amplified spontaneous emission of that laser drives the $3 p-3 d$ transition, while the output of the oscillator populates an $n f$ Rydberg state. The dye laser beams are sent to the vacuum chamber and focused to less than 1-mm diameter spots where they cross each other and the atomic beam. Unless stated otherwise, the laser field and microwave field are polarized vertically throughout the experiment.

\subsubsection{Experimental Observations}

\section{Lithium}

The main objective of the described experiment is to show how a presence of non-zero static fields affects ionization of Rydberg atoms. Namely, a static field can enhance the ionization process, or suppress the production of extremely high-lying states as discussed in detail in Chapters 4 and 5. The first measurements of ionization thresholds at $17 \mathrm{GHz}$ were done for higher states of lithium Rydberg atoms. To determine a fraction of atoms left in any bound state after the microwave pulse, we tune the laser to a specific Rydberg state, 

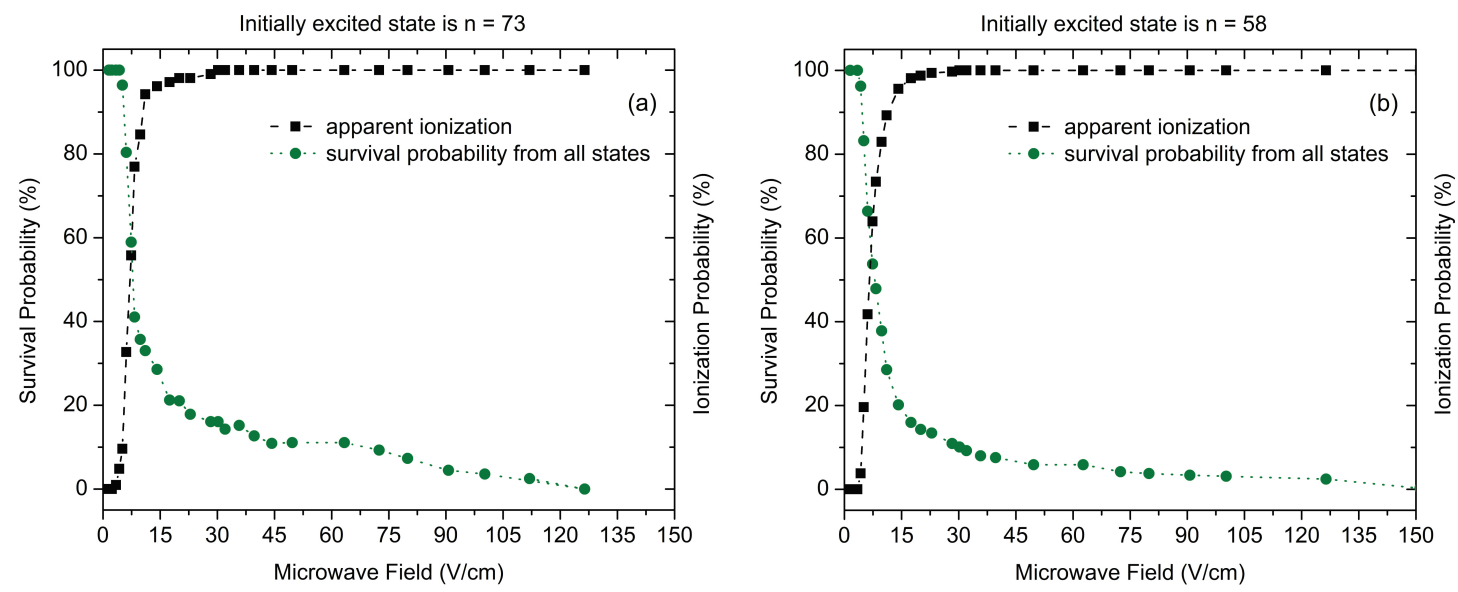

Figure 3.2: Microwave ionization of the Li $73 p(\mathrm{a})$ and $58 p$ (b) state. Fraction of atoms surviving the MW field as a function of its strength detecting electrons from all bound states of $n \geq 55$ (circles) and fraction of atoms apparently ionized by the MW field as a function of its strength observed by detecting ions with the positive FIP (squares). The apparent microwave ionization signal collected with the positive FIP is due to both microwave ionization and field ionization of atoms in state of $n \geq 90$. Because the high-lying states are present in both signals, they are not complementary.

and, using a negative FIP and electron detection, record the electron signal as a function of the microwave field amplitude. Due to a time delay between the end of the microwave pulse and the FIP, all free electrons created as a result of microwave or photoionization leave the interaction region before the FIP, so, in this case a survival probability is measured. To compare our results to the previous microwave ionization experiments, we, as well, detect ions with a positive FIP, the amplitude of which is not high enough to field ionize the initial state, but ionizes atoms with $n>90$. With a positive FIP, population trapped in the extremely high-lying states is indistinguishable from ions due to microwave ionization, so we term the positive FIP signal due to these two sources the apparent microwave ionization signal. We recorded such data for several Rydberg states, and show two characteristic plots in Fig. 3.2 (a) for $n=73$ and (b) $n=58$. The black squares in Fig. 3.2 represent the apparent microwave ionization signal obtained by detecting ions with the positive FIP, while the green circles represent the surviving atom signals obtained by detecting electrons from all bound states of $n \geq 55$ with a negative FIP. All the negative FIP signal (green circles) at microwave fields over $30 \mathrm{~V} / \mathrm{cm}$ in Fig. 3.2 is due to high-lying states.

The observed $50 \%$ ionization threshold for the $n=73$ state of $\mathrm{Li}(\Omega=1)$ is in a good agreement with the prediction of Jensen et al. [25] that the ionization fields in the 
region $\Omega \geq 1$ are independent of the principle quantum number and can be calculated as $E=2.4 \omega^{5 / 3}$ (Eq. 1.15). On the other hand, a survival probability curve for any measured state, in contrast to the apparent ionization, yields a significant population left bound in very high-lying states as will be explicitly shown in Chapter 5. Thus, the ion detection method does not provide the correct information about the fraction of population ionized by the microwave field or left in a bound state. The ionization of $\mathrm{Li}$ atoms by $17-\mathrm{GHz}$ fields yielded a population left trapped in the high-lying states, so we decided to change the excitation scheme to study ionization of $\mathrm{Na}$ atoms once more. The results that we obtained showed not only the production of high-lying states, but also a substantial deviation from the results of previously conducted experiments.

\section{Sodium}

In the following, the $3 d-n f$ laser was fixed at several different states, and survival and ionization probabilities were taken as described in the previous subsection. First, we show the observed ionization curves for $n=73 f$ state of $\mathrm{Na}$ taken in the presence of a small $10-\mathrm{mV} / \mathrm{cm}$ static field. As can be seen in Fig. 3.3 (a), ionization of $\mathrm{Na}$ atoms is similar to ionization of $\mathrm{Li}$, and we find a substantial fraction of population left in the high-lying states. That result differs from the observations of previous microwave ionization experiments on $\mathrm{Na}$ at this frequency and will be explained in subsequent chapters. Again, the apparent microwave ionization signal constitutes both ions created in microwave ionization and field ionization of states with $n \geq 90$, and two curves in Fig. 3.3 (a) are not complimentary. We repeated the same measurement with the static field of $35 \mathrm{mV} / \mathrm{cm}$ present that ionizes the high-lying states as will be discussed in Chapter 5. Fig. 3.3 (b) shows that, in the absence of the high-lying states, two detection methods yield the same result.

In Fig. 3.4 we show the apparent microwave ionization signals obtained with the positive FIP by filled circles, triangles, and squares for the $24 f, 30 f$, and $40 f$ states, respectively. The static field is in all cases $10 \mathrm{mV} / \mathrm{cm}$. It is useful to compare the microwave ionization thresholds of Fig. 3.4, to those reported by Pillet et al. [13]. In their work, with the laser polarization parallel to the microwave field only $n d$ states of $|m|=0$ and 1 were excited, 

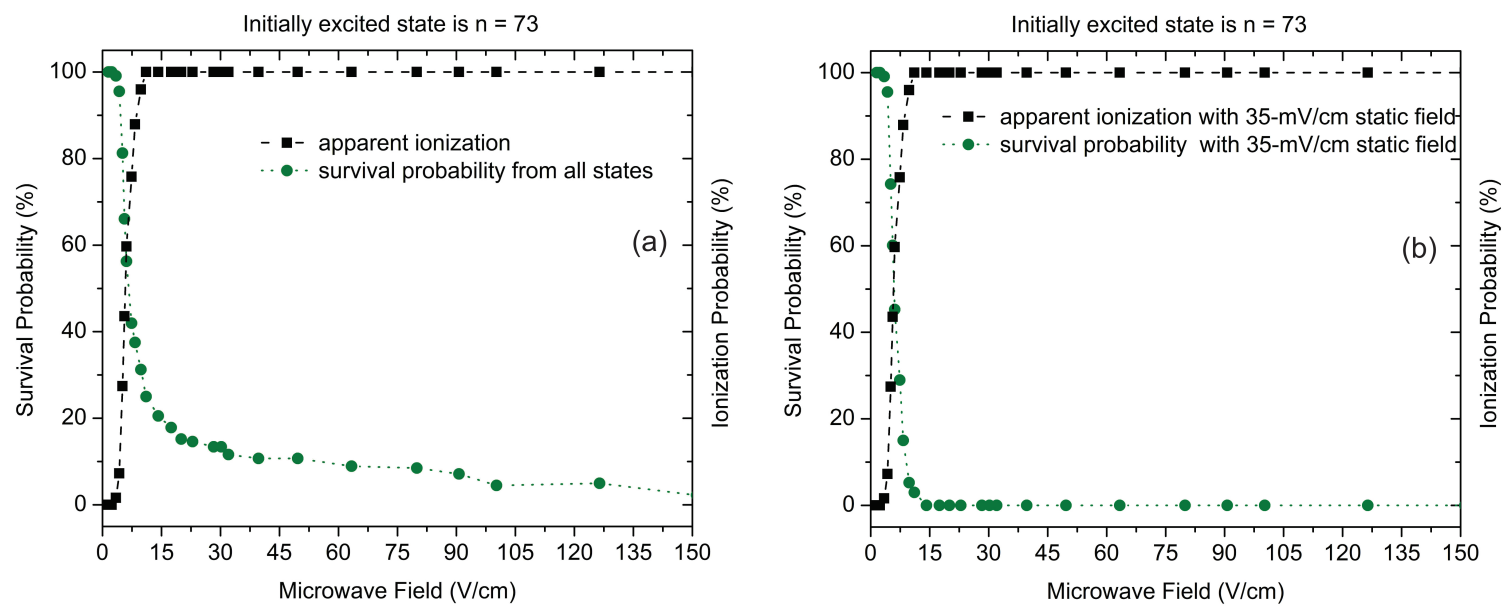

Figure 3.3: Microwave ionization of the Na $73 f$ state. Fraction of atoms surviving the MW field as a function of its strength detecting electrons from all bound states of $n \geq 55$ (circles) and fraction of atoms apparently ionized by the MW field as a function of its strength observed by detecting ions with the positive FIP (squares). Data is taken in the presence of $10-\mathrm{mV} / \mathrm{cm} \mathrm{(a),} \mathrm{and} 35-\mathrm{mV} / \mathrm{cm}$ (b) static field. The apparent microwave ionization signal collected with the positive FIP is due to both microwave ionization and field ionization of atoms in state of $n \geq 90$. Because the high-lying states are present in both signals, they are not complementary an (a). However, when the static field is added, both detection methods yield the same result.

and one threshold field for ionization was observed, with $50 \%$ ionization at $E=0.28 n^{-5}$, a field slightly lower than the Inglis-Teller field of $1 / 3 n^{5}$. The Inglis-Teller field is the field at which the $|m|=0$ Stark states of $n$ and $n+1$ intersect. For $|m|=0$ and 1 the $n$ and $n+1$ energy levels do not cross but exhibit substantial avoided crossings due to the large quantum defects, 1.35 and 0.85 , of the $s$ and $p$ states. Ionization of $|m|=0$ and 1 states occurs at the field $E=1 / 3 n^{5}$ since a microwave field of this amplitude samples the avoided crossing between the $n$ and $n+1$ levels, allowing the $n \rightarrow n+1$ transition to occur. The coherent effect of multiple cycles is required, and it is a resonant process [55]. Similar transitions through higher lying levels occur, culminating in ionization. The vertical dashed lines in Fig. 3.4 mark the $50 \%|\mathrm{~m}|=0$ and 1 threshold fields measured by Pillet et al. With the laser polarization perpendicular to the microwave field, so as to excite $|m|=0,1$, and 2 levels, they observed two thresholds, the $|m|=0$ and 1 threshold at $E \cong 1 / 3 n^{5}$, and a threshold at $E=1 / 9 n^{4}$, the static ionization field for the red hydrogen Stark state. In other words, the $\mathrm{Na}|m|=2$ states ionize by field ionization of the initial $n$ state. Transitions through higher lying states do not occur since the largest $|m|=2$ 


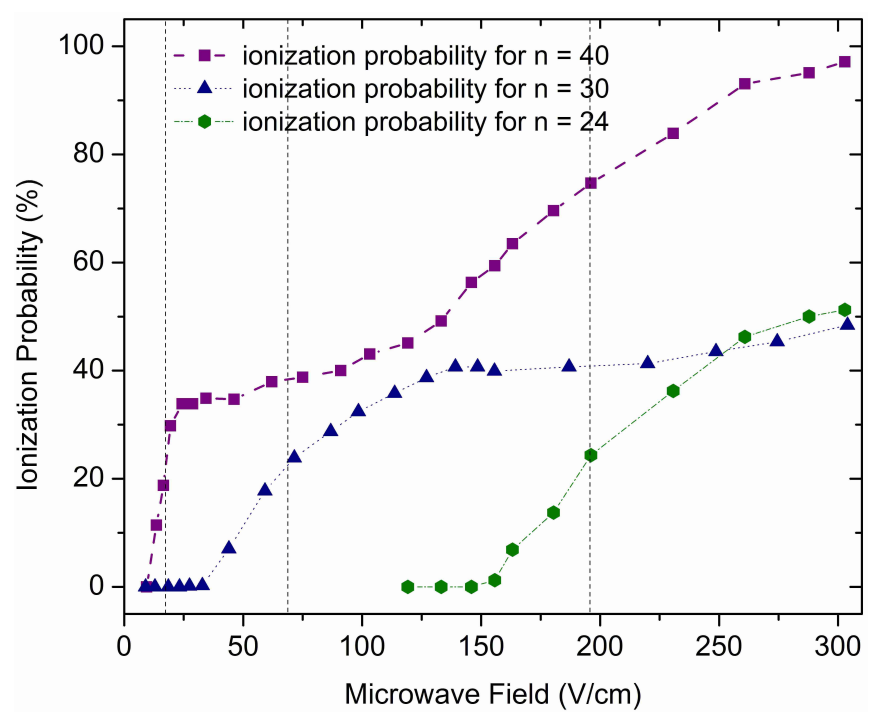

Figure 3.4: Fractional apparent ionization for the Na $24 f$ (filled circle), $30 f$ (filled triangle), and $40 f$ (filled square) states obtained with a positive FIP. Vertical lines correspond to the $50 \%$ ionization threshold of $|m|=0$ and 1 states measured by Pillet et al. [13]. The $40 f$ state clearly exhibits two thresholds, while lower $30 f$ and $24 f$ states have one threshold, but do not reach the second one, as not the $100 \%$ of atoms are ionized.

quantum defect is the $d$ quantum defect of 0.015. Returning to Fig. 3.4, we see that the $24 f$ and $30 f$ ionization curves have ionization thresholds at $E=1 / 3 n^{5}$, as expected, but the $40 f$ curve exhibits two thresholds, not one. The first is at $E \cong 1 / 3 n^{5}$, as expected, and the second is near $E=1 / 9 n^{4}=223 \mathrm{~V} / \mathrm{cm}$, the hydrogenic ionization field. We attribute the first $40 f$ threshold to $|m|=0$ and the second to $|m|=1$, ionizing by field ionization, as previously observed for $|m|=2$. While the $24 f$ and $30 f$ signals of Fig. 3.4 exhibit the expected threshold fields, they differ from those of Pillet et al. in that at most $40 \%$ ionization is observed, not $100 \%$.

From Fig. 3.4 alone it is not apparent that the $24 f$ and $30 f$ ionization signals reach only $40 \%$ ionization, but it becomes apparent when we add a static field. Fig. 3.5 shows $40 f$, $30 f$, and $24 f$ ionization threshold curves taken for several static fields. For instance, as the static field is raised from $10 \mathrm{mV} / \mathrm{cm}$ to $1.5 \mathrm{~V} / \mathrm{cm}$, the $|m|=1$ ionization threshold moves from $E=1 / 9 n^{4}$ to $E=1 / 3 n^{5}$ for $30 f$. The curve taken at $1.5 \mathrm{~V} / \mathrm{cm}$ is the same as observed by Pillet et al. The $40 f$ and $24 f$ states require smaller and larger static fields, respectively, to reach $1 / 3 n^{5}$ limit as shown in Fig. 3.5 (a) and (c). The observations shown in Figs. 3.4 and 3.5 provide clear evidence that static fields on the order of $1 \mathrm{~V} / \mathrm{cm}$ were present 

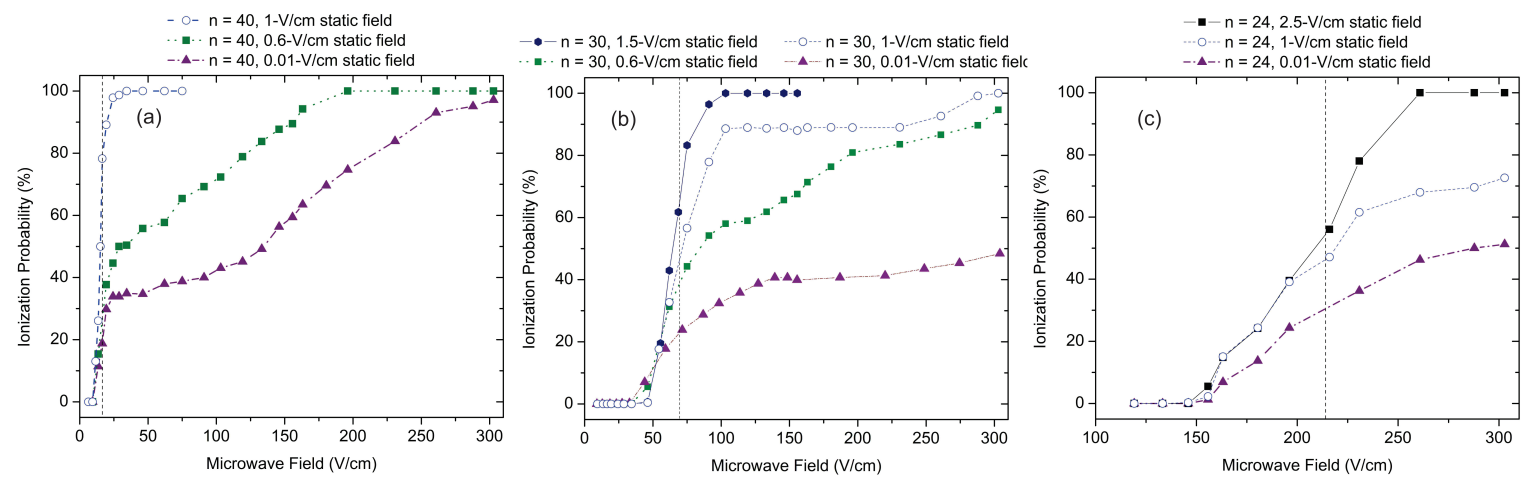

Figure 3.5: $\quad \operatorname{Na} 40 f(\mathrm{a}), 30 f(\mathrm{~b})$, and $24 f(\mathrm{c})$ signal obtained with the positive FIP vs microwave field for static field $E_{s}$ values from $10 \mathrm{mV} / \mathrm{cm}$ to $2.5 \mathrm{~V} / \mathrm{cm}$. The vertical dotted lines correspond to the $50 \%$ ionization thresholds as measured by Pillet et al. [13] at $0.28 n^{5}$ for $n=40,30$, and 24 . For $30 f$, with $E_{s}=10$ $\mathrm{mV} / \mathrm{cm}$ the $|m|=0$ threshold is roughly $68 \mathrm{~V} / \mathrm{cm}$, and the $|m|=1$ threshold is at $E=1 / 9 n^{4}=700 \mathrm{~V} / \mathrm{cm}$, too high a field to be observed. As the static field is raised, the $|m|=1$ threshold moves to join the $|m|=0$ threshold at $E=1 / 3 n^{5}$.

in the early microwave ionization experiments done in waveguide cavities. To emphasize the importance of the static field for microwave ionization, we show Fig. 3.6, in which, as functions of the static field, negative and positive FIP signals for the $30 f$ state exposed to a $120-\mathrm{V} / \mathrm{cm}$ microwave field are plotted. The solid line shows the high-lying state $(n>250)$ signal observed, which has a half width of $20 \mathrm{mV} / \mathrm{cm}$. In contrast, the rise in the microwave ionization signal due to the $|m|=1$ states, shown by the broken line, has a half width of $800 \mathrm{mV} / \mathrm{cm}$.

A general result of the measurements described above is the realization that stray fields almost certainly altered the $\mathrm{Na}$ microwave ionization threshold fields in previous experiments. Both $|m|=0$ and 1 states were thought to ionize at $E=1 / 3 n^{5}$. Now it appears that the $|m|=1$ states ionize by field ionization, at $E=1 / 9 n^{4}$, unless there is a field present, in which case ionization can occur at a microwave field as low as $E=1 / 3 n^{5}$. This effect was first observed by Pillet et al. [18], who found the same phenomenon in the microwave ionization of $\mathrm{Li}|m|=0$ states, which have 0.35 as the only quantum defect greater than 0.05. The $\mathrm{Na}|m|=1$ states are somewhat similar; 0.85 is the only quantum defect larger than 0.015 . Why the small static field reduces the microwave ionization field from $1 / 9 n^{4}$ to $1 / 3 n^{5}$ can be understood as follows. The $n \rightarrow n+1$ transition is a resonant multiphoton transition which can be envisioned as being due to the near degeneracy of the dressed, or 


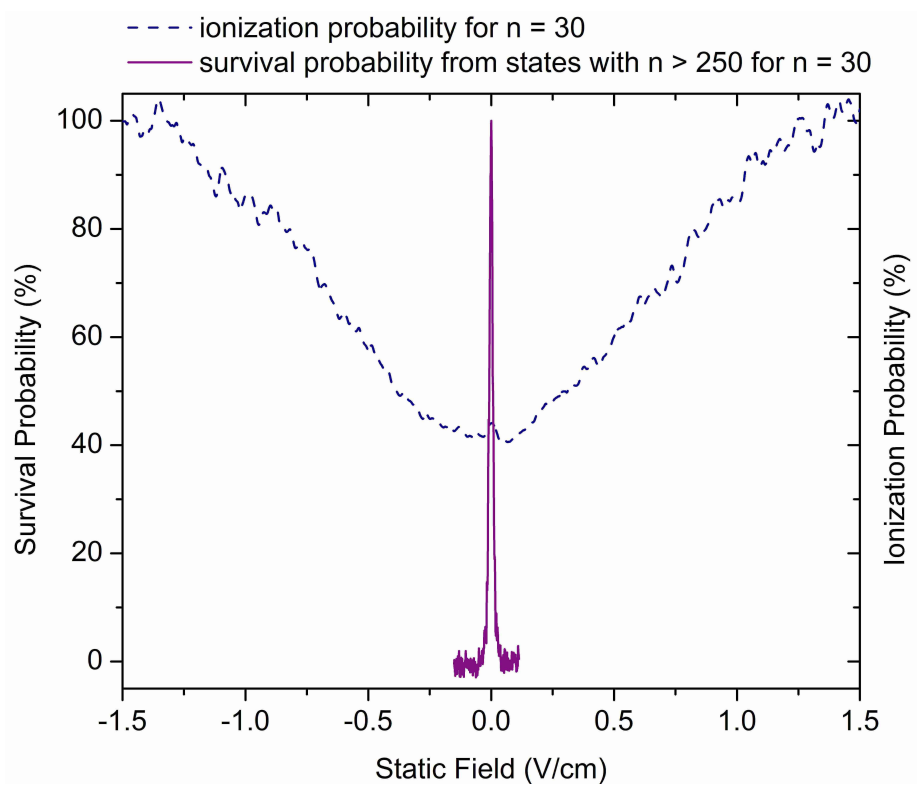

Figure 3.6: The static field dependence of the high-lying state signal and the microwave ionization probability of the $30 f$ after exposure to a $120-\mathrm{V} / \mathrm{cm}$ microwave pulse. The high-lying state signal observed by detecting electrons with a negative FIP (solid line). The microwave ionization probability observed by detecting ions with a positive FIP (dashed line). The half widths of the two curves differ by a factor of 40 .

sideband, states formed from the Stark states of $n$ and $n+1$. If the quantum defect is small, the multiphoton Rabi frequency is as well, and the $n \rightarrow n+1$ transition does not occur unless the microwave field is almost perfectly resonant with the atomic transition. In zero static field the dressed, or sideband states, of the same $n$ are degenerate, but adding a small electric field lifts the degeneracy, and the sideband states from different Stark states form a quasi continuum, allowing the resonance condition to be met. Their experiment showed that the static field $E_{s}$ required is that which shifts the extreme Stark state by half the microwave frequency, i. e. $E_{s}=\omega / 6 n^{2}$. Applying this requirement to $n=30$ with a frequency $\omega / 2 \pi=16.9 \mathrm{GHz}$ yields $E_{s}=1.5 \mathrm{~V} / \mathrm{cm}$, in good agreement with Fig. 3.6. Apparently the Na $p$ quantum defect alone is not large enough to allow microwave ionization at $E=1 / 3 n^{5}$, unless there is a field present to convert the dressed, or sideband, states from the Stark states into a quasi continuum. The realization that there was a field of approximately 1 $\mathrm{V} / \mathrm{cm}$ present in the earlier experiment shows that microwave ionization of the $\mathrm{Na}|m|=2$ states is unaffected by this field. Apparently the field induced quasicontinuum is too coarse grained for the extremely small $|m|=2$ avoided crossings, and the multiphoton transitions 
do not occur.

\subsection{Ionization of Li Rydberg atoms by 38-GHz microwave fields}

Below we present an investigation of microwave ionization of $\mathrm{Li}$ by $38.3 \mathrm{GHz}$ microwave fields, from the photoionization limit, $n>420$, where $\omega>1 / 2 n^{2}$ and $\Omega>210$, to $n=37$, where $\omega \simeq 0.32 \omega_{K}$ and $\Omega=0.32$. We find a smooth variation in the fields at which $10 \%$ and $50 \%$ ionization occur, in agreement with previous experiments, in which pieces of the above range were explored. More surprising, we find that for all initial states a substantial fraction, $\sim 5 \%$, of the atoms is found in high-lying Rydberg states subsequent to a 200-ns long microwave pulse. In the sections which follow we describe our experimental approach, present our observations and discuss their implications.

\subsubsection{Experimental Approach}

A thermal beam of $\mathrm{Li}$ atoms in its ground state passes through the antinode at the center of a 38.3-GHz Fabry-Perot cavity where the atoms are excited to a Rydberg state by three 20 -ns dye laser pulses via $2 s \rightarrow 2 p \rightarrow 3 s \rightarrow n p$ transitions. The excited atoms are then exposed to a microwave field pulse, as shown in the timing diagram in Fig. 3.7. The microwave pulse starts about $50 \mathrm{~ns}$ after the laser excitation and lasts for $200 \mathrm{~ns}$. After the end of the microwave pulse, typically $300 \mathrm{~ns}$ later, atoms are field ionized by a slowly rising field pulse. The electrons ejected by the ionization field are detected by a dual microchannel plate detector. The time resolved MCP signal tells us how many atoms were not ionized by the microwave pulse as well as their final state distribution. The MCP signal is captured by a gated integrator or oscilloscope and recorded for later analysis.

The microwave cavity consists of two brass mirrors $40.5 \mathrm{~mm}$ in diameter with $75.9-\mathrm{mm}$ radii of curvature. The on-axis spacing between mirrors is $44.5 \mathrm{~mm}$. The cavity is operated on the $\mathrm{TEM}_{012}$ mode at a frequency of $38.34 \mathrm{GHz}$ with $Q=5100$. The microwave system generates a 38.34- $\mathrm{GHz}$ pulse with 0 - to $90 \mathrm{~V} / \mathrm{cm}$ amplitude and a variable width. All 


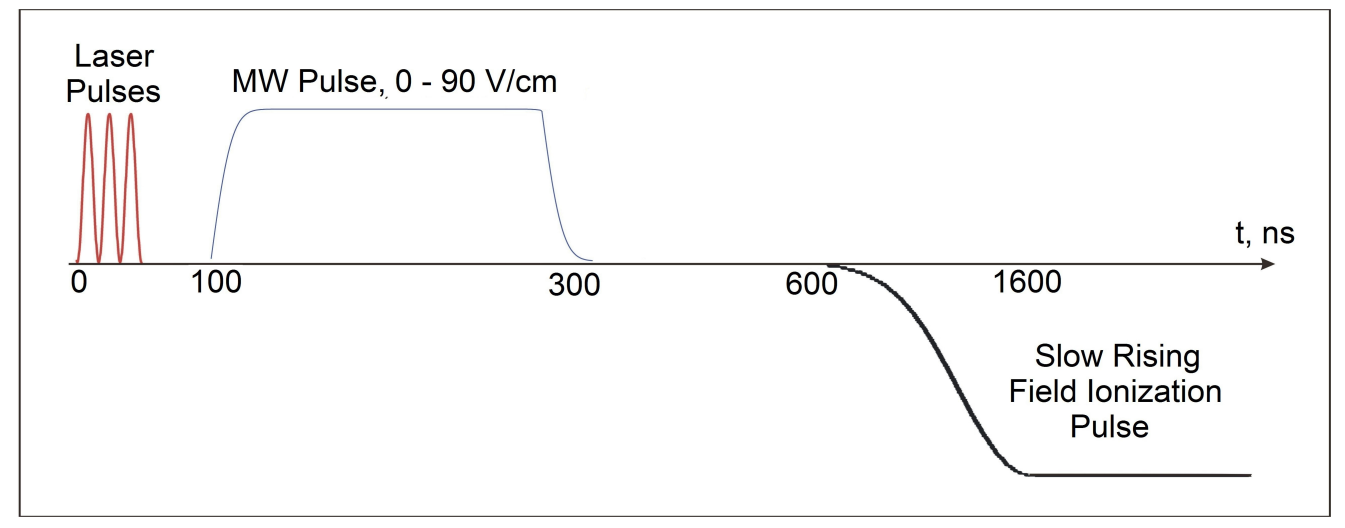

Figure 3.7: Experimental timing diagram for the investigation of the microwave ionization of Li by a 38.34-GHz microwave field.

apparatus is triggered at a $500 \mathrm{~Hz}$ repetition rate by a frequency-doubled Nd:YLF laser. The linewidth of the laser making the last transition is about $8 \mathrm{GHz}$, and its frequency can be tuned to populate any Rydberg state of $n>35$. The three laser beams are sent to the vacuum chamber and focused to less than 1-mm diameter spots where they cross each other and the atomic beam. The laser field and microwave field are polarized vertically throughout the experiment.

\subsubsection{Experimental Observations}

The objective of this work was to study the ionization thresholds of Li Rydberg states in a microwave field of $38 \mathrm{GHz}$ not only near the ionization limit $(\Omega \rightarrow \infty)$ [23], but also in the region where the classical orbital Kepler frequency of the Rydberg states is larger than the microwave frequency of $38.3 \mathrm{GHz}(\Omega<1)$. In order to observe the ionization behavior, we continuously change the binding energy of Rydberg states by scanning the frequency of the laser driving the $3 s \rightarrow n p$ transition while recording the field ionization signal from the MCP. We repeat such scans in zero field, when the microwaves are off, as well as in the presence of microwave fields of different amplitudes. Obtaining these spectra for many microwave fields allows us to extract the threshold fields required to ionize a given fraction of the initial population. Unless stated otherwise, data are taken for 200-ns long, 38.34-GHz microwave pulses, in such a way that the signal from all bound states ionized by the FIP is recorded by the gated integrator. Continuously changing the initial binding energy of the 
excited atoms, by scanning the laser frequency, we obtain spectra showing the population found in bound states after the microwave pulse. Fig. 3.8 shows spectra recorded with the microwave field off (a), as well as spectra obtained in the presence of fields of $36 \mathrm{~V} / \mathrm{cm}$ (b) and $90 \mathrm{~V} / \mathrm{cm}(\mathrm{c})$. The zero of energy of the horizontal scale is the ionization limit.
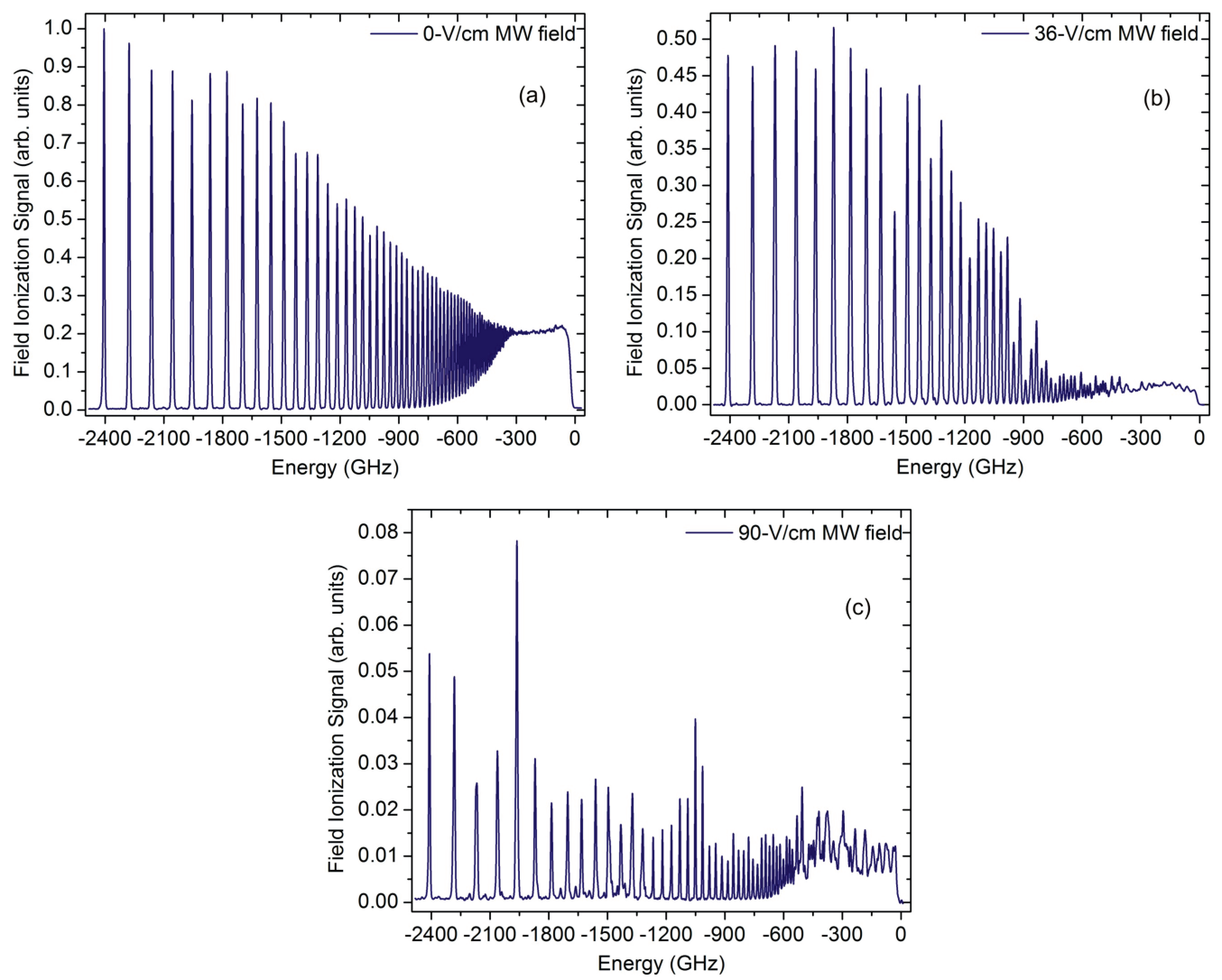

Figure 3.8: Field ionization signal as a function of the laser frequency tuning, in terms of energy relative to the ionization limit (IL), after exposure to a microwave pulse of amplitude: (a) $0 \mathrm{~V} / \mathrm{cm}$, (b) $36 \mathrm{~V} / \mathrm{cm}$ and (c) $90 \mathrm{~V} / \mathrm{cm}$. The horizontal axis is calibrated by the etalon and optogolvanic signals. The first peak on the graphs corresponds to $n=37$, at $-2410 \mathrm{GHz}$.

In zero field we observe resolved bound Rydberg states at energies lower than $-300 \mathrm{GHz}$ and observe a flat signal at higher energies, where the spacing between adjacent levels is smaller than the linewidth of our laser, which occurs at $n \approx 100$. A delay between the laser excitation and the start of the FIP ensures that free electrons created by photoionization leave the interaction region and are not detected by the FIP. Consequently, at an energy of $-45 \mathrm{GHz}$, the signal starts falling to zero because photoelectrons are not detected when the laser is tuned over the ionization limit. Energies are measured relative to the zero field limit. 
Due to the finite linewidth of the laser, the signal drops to zero with a finite slope, and we denote the middle of that slope as the depressed ionization limit. The limit is depressed due to a nonzero static field in the interaction region. We estimate that stray field using $\Delta W=2 \sqrt{E}$. Typically, the data are taken when the depressed ionization limit is at -18 $\mathrm{GHz}$, which corresponds to a stray field of $9 \mathrm{mV} / \mathrm{cm}$. In the $36-\mathrm{V} / \mathrm{cm}$ scan of Fig. 3.8 we see a clear drop in the survival probability at an energy of $-900 \mathrm{GHz}, n=60$, several states above the point where the classical orbital frequency of the atom most closely matches the microwave frequency, i. e. where $\Omega=1$. The match occurs at $n=56$ which has an energy of $-1050 \mathrm{GHz}$. The $90-\mathrm{V} / \mathrm{cm}$ scan reveals several notable features. First, there is a state at $-1960 \mathrm{GHz}(n=41)$ that is much more resistive to ionization than the adjacent states. Second, states at energies near $-1050 \mathrm{GHz}$, where the microwave frequency matches the Kepler frequency, are remarkably stable. Third, a large number of atoms are left in bound states after a $200-\mathrm{ns}, 90-\mathrm{V} / \mathrm{cm}$ pulse. For comparison, we note that a $90-\mathrm{V} / \mathrm{cm}$ static field would ionize states down to an energy of $-1300 \mathrm{GHz}$. Finally, there is very noticeable modulation at the microwave frequency just below the limit, which agrees with previous observations [23]. This modulation is most apparent in Fig. 3.8 (c).

Fig. 3.8 shows only typical spectra obtained for several microwave fields. From the set of all such spectra we can extract the threshold fields for ionization. Taking spectra in the microwave field and normalizing them by the zero microwave field signal at the same laser frequency, we are able to determine the fraction of atoms surviving vs microwave field amplitude for each Rydberg state. Here, we present such data for the range $37<n<\infty$, which encompasses $1 / 3 \leq \Omega<\infty$. In Fig. 3.9 (a) we show the microwave fields required to ionize $10 \%$ and $50 \%$ of the initial population vs binding energy.

In Fig. 3.9 (a) we show the calculated ionization fields. At low $n$, where $1 / 3<\Omega<1$, we show the $10 \%$ ionization fields calculated by Krug and Buchleitner [22]. When $\Omega<1$ the finite size of the $\mathrm{Li}^{+}$core leads to ionization fields far lower than those observed in $\mathrm{H}$, but for $\Omega>1$, the presence of a finite sized core is inconsequential, and the calculated ionization fields for $\mathrm{Li}$ and $\mathrm{H}$ are the same. For example, scaling the experimental hydrogenic $36 \mathrm{GHz}$ ionization fields to $38 \mathrm{GHz}$ at $n=37$ yields an ionization field of $125 \mathrm{~V} / \mathrm{cm}$ [53], five times 

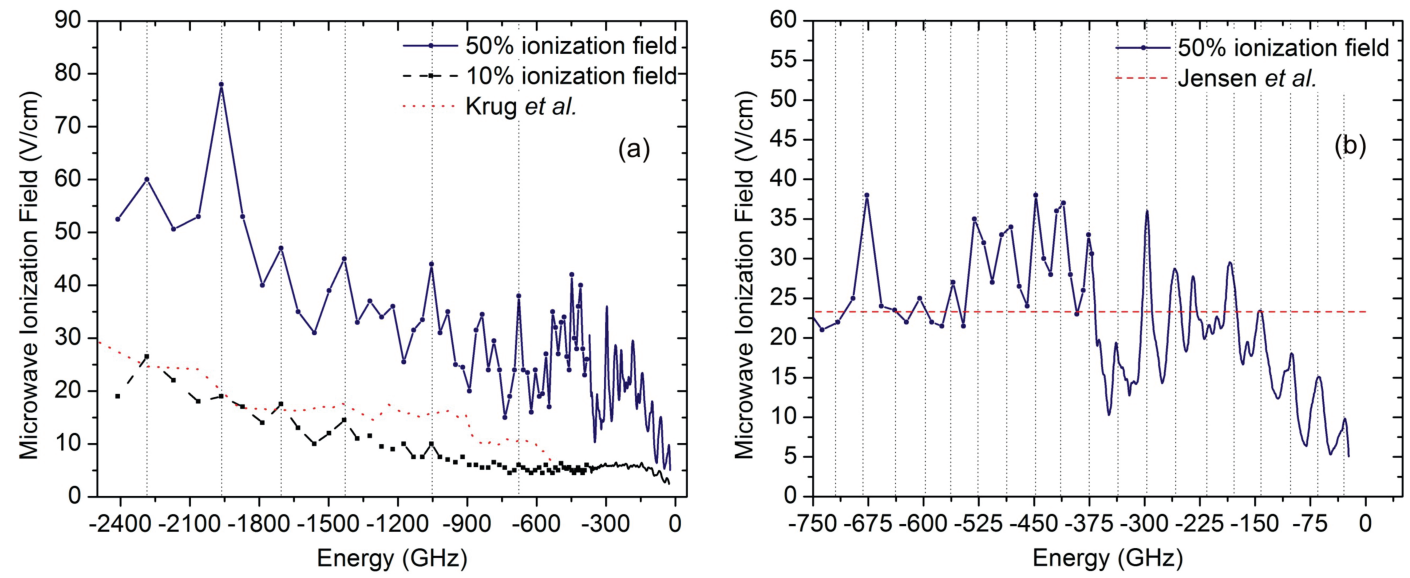

Figure 3.9: (a) MW fields as a function of atomic energy for $50 \%$ and $10 \%$ ionization. Vertical dotted lines on graphs correspond to the energies of Rydberg states for which the classical Kepler frequency equals $3 \omega$, $2.5 \omega, 2 \omega, 1.5 \omega, \omega$, and $0.5 \omega$ respectively, where $\omega=38.34 \mathrm{GHz}$ is the MW frequency. Our observations for the $10 \%$ field are in good agreement with theoretical calculations made by Krug and Buchleitner for the ionization of Li by a 36-GHz MW field (red dotted line) for $1 / 3<\Omega<1$. To our knowledge, no calculation exists for $10 \%$ ionization fields at $38.3 \mathrm{GHz}$ in the region from $-500 \mathrm{GHz}$ to the IL. (b) An expanded view of the $50 \%$ ionization fields in the $\Omega>1$ regime. Our result is in excellent agreement with a prediction of Jensen et al. [25] (red dashed line) that the average ionization field does not depend on $n$. That prediction, however, is not intended to reproduce the experimental structure that we observe. As shown by the vertical dotted lines spaced by $\omega$, the structure in the ionization fields is at the microwave frequency.

higher than the $\mathrm{Li} 10 \%$ ionization field. In contrast, the scaled $\mathrm{H} n=56$ field is $19 \mathrm{~V} / \mathrm{cm}$, in good agreement with the $\mathrm{Li} 10 \%$ ionization field. The intermediate regime, $1 / 3<\Omega<1$, where the effect of the core is changing, poses the greatest theoretical challenge, and for this reason the agreement between the calculation of Krug and Buchleitner and our observations is most impressive.

To a first approximation, we observe an $n$ independent ionization field for $\Omega>1$ for both the $10 \%$ and $50 \%$ ionization fields. Jensen et al. [25] proposed that in the $\Omega>1$ regime ionization occurs by a sequence of transitions through excited states. As discussed in Section 1.2.1 (Eqns. 1.15-1.17), the requirement is that the dipole coupling due to the microwave field equals the average detuning from resonance of the transitions of the sequence. Since both the coupling and the detuning scale as $1 / n^{3}$, this condition is met for all $n$ when $E=2.4 \omega^{5 / 3}$. This prediction, shown by the red dashed line in Fig. 3.9 (b), is in excellent agreement with the $50 \%$ ionization fields from $\Omega=1$ to within four microwave photons of the ionization limit.

When the laser frequency is fixed, we can record an oscilloscope trace of a signal coming 

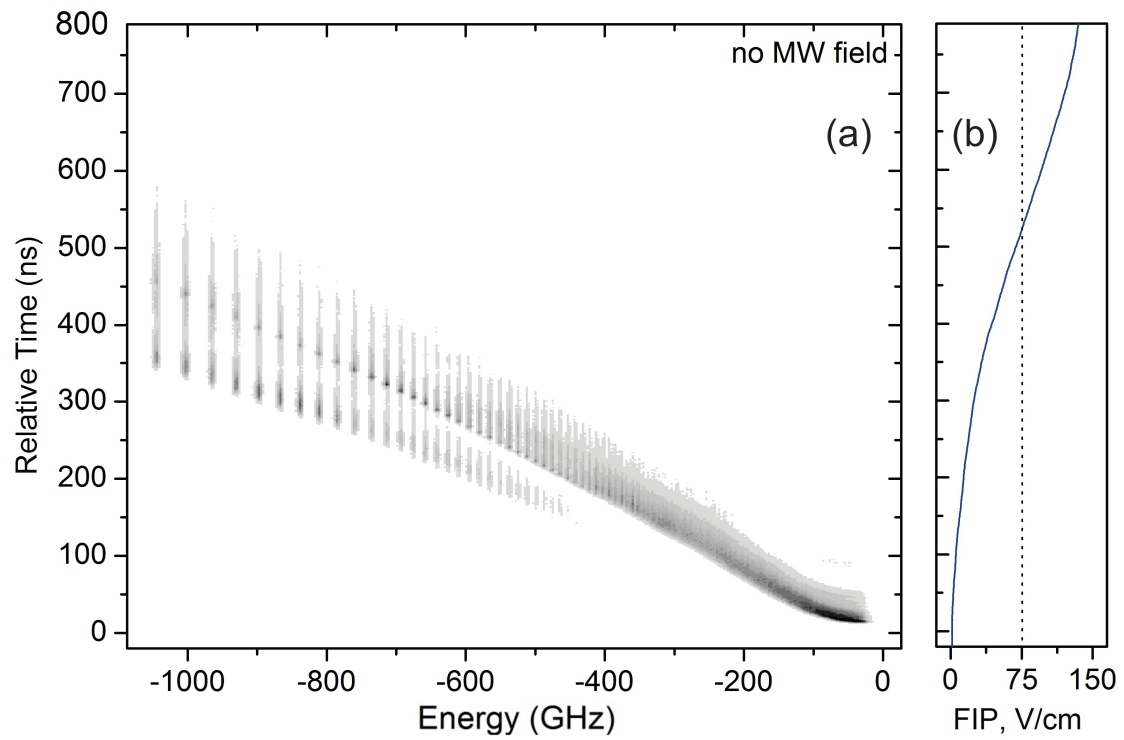

Figure 3.10: (a) Rydberg state distribution as a function of energy with the microwave field off. The distribution shown are gray scale representations of oscilloscope traces of time resolved field ionization signals. Black is a large signal and white is no signal. (b) The FIP is shown on the same vertical time scale. Rydberg states ionize at two different regimes: adiabaticaly at $1 / 16 n^{4}$ and diabaticaly at $1 / 9 n^{4}$.

from the MCP. As we utilize a slow-rising FIP, atoms with different Rydberg energies ionize at different voltages of the FIP which results in peaks separated in time on the oscilloscope. Thus, if we slowly scan the laser frequency and let the computer record an oscilloscope trace for each step, we can obtain the final state distribution of atoms after the microwave pulse. Then, the oscilloscope trace taken for each laser frequency is mapped to a gray scale with black corresponding to large signal amplitude, and white to no signal. First, we obtain a map of state distribution of Rydberg atoms with the microwave field off which is shown in Fig. 3.10. Here, the vertical scale is time at which the signal appears on the oscilloscope. For convenience, in Fig. 3.10 (b), we also show the FIP. It is evident in Fig. 3.10 (a) that, close to the ionization limit, Rydberg $n p$ states ionize diabaticly by the field $E=1 / 9 n^{4}$. Around $-400 \mathrm{GHz}$ below the limit, atoms start ionizing adiabaticaly at $E=1 / 16 n^{4}$, a regime that becomes more dominant for lower states, as was discussed by Noel et al. [56].

Our main interest is to compare the final state distribution of atoms after the microwave pulse to the initial state distribution. Fig. 3.11 shows the final state distributions of atoms that survive the microwave fields vs laser tuning for two characteristic field amplitudes. The vertical scales of Fig. 3.11 are given both as time and the field of the FIP. Figs. 3.11(a) 


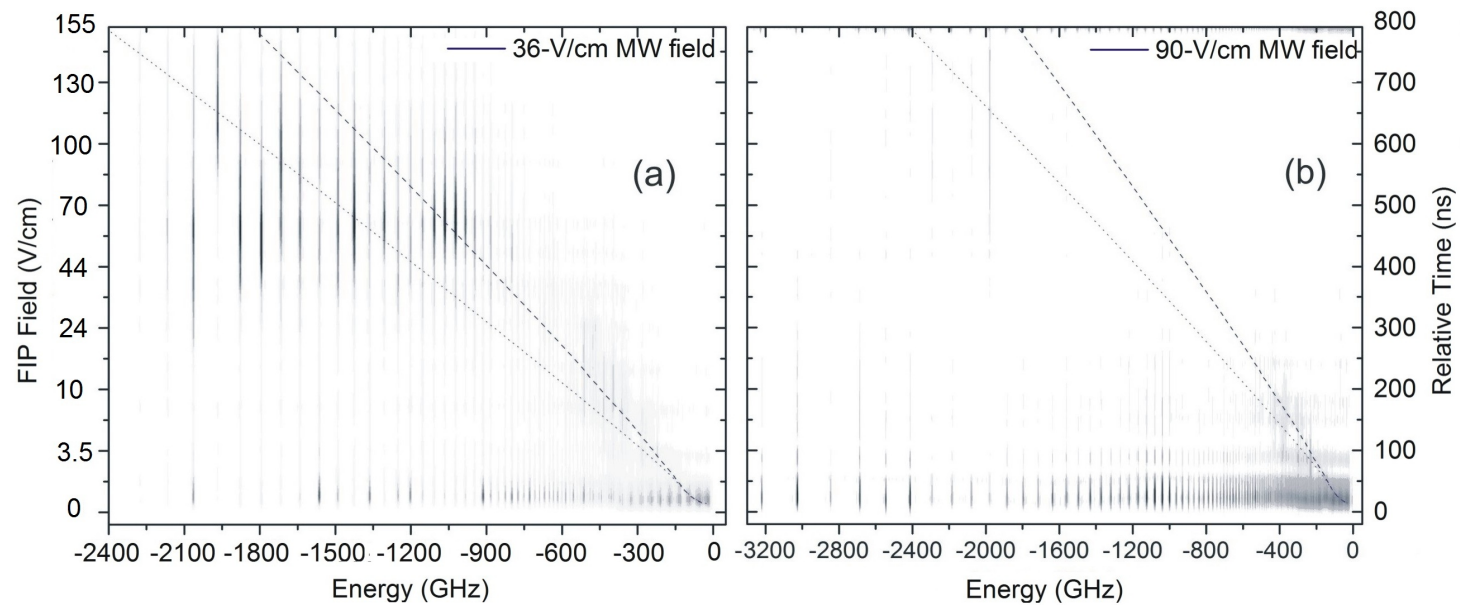

Figure 3.11: Final state distributions as a function of energy for: (a) $36-\mathrm{V} / \mathrm{cm}$, (b) $90-\mathrm{V} / \mathrm{cm}$ MW fields. The distributions shown are gray scale representations of oscilloscope traces of time resolved field ionization signals. Black is a large signal and white is no singal. For convenience the vertical scale is shown both as time and the field of the FIP. The dashed lines in (a) and (b) show the diabatic ionization field $\left(1 / 9 n^{4}\right)$, while the dotted lines correspond to the adiabatic ionization field $\left(1 / 16 n^{4}\right)$. Both 36 and $90-\mathrm{V} / \mathrm{cm}$ plots show the presence of the extremely high-lying states that are ionized within the first $50 \mathrm{~ns}$ of the FIP. The redistribution to states other than the initial or extremely high-lying occurs to both higher and lower binding energies, but in no case do we observe a continuous distribution from the initial state to the IL. In (b) an additional peak set between 70 and $100 \mathrm{~ns}$ is due to electrical ringing and is an artifact of the experiment.

and (b) show the final state distributions obtained with 36 and $90-\mathrm{V} / \mathrm{cm}$ microwave fields. The adiabatic and diabatic ionization fields for the initially excited states are shown by the broken and dotted lines, respectively. An unexpected discovery is evidence of the extremely high-lying states that are produced by the $38-\mathrm{GHz}$ microwave field for all initial states. The signal from these states is seen at ionizing fields between 0 and $2 \mathrm{~V} / \mathrm{cm}$ (times between 10 and $50 \mathrm{~ns}$ ) in Fig. 3.11. Both $36-\mathrm{V} / \mathrm{cm}$ and $90-\mathrm{V} / \mathrm{cm}$ scans in Fig. 3.11 show the presence of extremely high lying states for initial energies down to $-2400 \mathrm{GHz}$ for $36-\mathrm{V} / \mathrm{cm}$ field, and $-3300 \mathrm{GHz}$ for the $90-\mathrm{V} / \mathrm{cm}$ field. Especially surprising is the $90 \mathrm{~V} / \mathrm{cm}$ scan, as we see that almost all other population is ionized, but weakly bound states are still present. That implies that bound atoms detected subsequent to the microwave pulse, as shown in Fig. 3.8 (c), are largely in highly excited states. This phenomenon will be discussed thoroughly in Chapter 5.

Other notable features in Fig. 3.11 are the following. First, we see in the $36-\mathrm{V} / \mathrm{cm}$ traces that atoms from the states with initial energies below $-900 \mathrm{GHz}$ can be found over a broad range of final energy. Second, final states for the laser tuning in range where 
$\Omega<1$ have their central maximum at the same time, corresponding to states adjacent to $n=56(-1050 \mathrm{GHz})$ where $\Omega=1$. In other words, atoms initially in lower lying states are apparently trapped in the states near $\Omega=1$. Very little population is found between pulsed ionization fields of 3 and $40 \mathrm{~V} / \mathrm{cm}$. However, for $\Omega \geq 1$, the pattern changes. Starting from an initial energy of $-950 \mathrm{GHz}$, just two states above where $\Omega=1$, most atoms, if not ionized, stay in the initial state and several adjacent ones or are found near the limit. In this regime the total bound population in that region drops abruptly which was also seen in Fig. 3.8 (b). Finally, we note that $n=36$ atoms, which have the anomalously high ionization field in Fig. 3.9 (a) are found near their initial energy with microwave fields of both 36 and $90 \mathrm{~V} / \mathrm{cm}$.

Above we discussed an investigation of microwave ionization, using the same apparatus, from low $n$, where $\Omega \approx 1 / 3$, to high enough $n$ that single photon ionization by the microwave field is possible. The fields required to effect $10 \%$ and $50 \%$ ionization decrease slowly with $n$ in the $\Omega<1$ regime and are almost constant in the $\Omega>1$ regime, except very close to the ionization limit, where pronounced structure is observed in the ionization fields, due to the atoms' being left in highly excited states. These observations are consistent with previous measurements which covered pieces of the range of $n$ explored here [24, 23].

\subsection{Ionization of $\mathrm{Na}$ Rydberg atoms at $80 \mathrm{GHz}$}

Following the experiments on 17 and $38-\mathrm{GHz}$ ionization of Rydberg atoms, it is interesting to investigate ionization mechanisms at $80 \mathrm{GHz}$. To our knowledge, a study at such a high frequency microwave field has not been conducted yet and might present results different from previous ionization experiments. The main difference between ionization at 15 and $80 \mathrm{GHz}$ is the size of the microwave photon and the resulting difficulty in fulfilling resonance conditions for transitions to higher Rydberg states. Ionization at $80 \mathrm{GHz}$ might present an intermediate regime between hydrogenic field ionization and the ladder mechanism in alkalis observed at fields below $20 \mathrm{GHz}$.

In the following we present results of an investigation of microwave ionization of $\mathrm{Na}$ 
Rydberg atoms by $79.05 \mathrm{GHz}$ microwave fields, from the photoionization limit to $n=25$, where $\Omega=0.19$. In the region $\Omega<1$, the observed ionization fields differ markedly from the results of experiments below $20 \mathrm{GHz}$. The ionization seems to happen through resonant transitions to higher states that can be, in some cases, enhanced significantly by the static field. In the following we discuss briefly the experimental approach, present our results, and discuss the conclusions.

\subsubsection{Experimental Approach}

A thermal beam of ground state sodium atoms crosses the microwave electric field antinode at the center of a $79.05-\mathrm{GHz}$ Fabry-Perot microwave cavity. There atoms are excited to a Rydberg state by the sequence of transitions $3 s \rightarrow 3 p \rightarrow 3 d \rightarrow n f$ induced by two 20-ns dye laser pulses and a continuous-wave diode laser, either in the presence of the microwave field or in zero-field, as shown in the timing diagram of Fig. 3.12 (a) and (b) respectively. In the latter case, after the laser excitation, atoms are subjected to a microwave pulse, typically 500-ns long. About $300 \mathrm{~ns}$ after the end of the pulse, atoms are exposed to a $1-\mu$ s rise time field ionization pulse. Depending on the polarity of the FIP, either electrons or ions are sent upwards to a dual microchannel plate detector. The MCP signal is amplified, captured by a gated integrator or oscilloscope, and recorded in a computer for later analysis. Unless states otherwise, an 800-V/cm FIP was used throughout this experiment, and it was capable of ionizing atoms in states with $n>25$.

The Fabry-Perot microwave cavity consists of two brass mirrors $69.7 \mathrm{~mm}$ in diameter with 52-mm radii of curvature. The on-axis spacing between mirrors is $90.51 \mathrm{~mm}$. The cavity is operated on the $\mathrm{TEM}_{048}$ mode at a frequency of $79.05 \mathrm{GHz}$ with $Q=8600$. The microwave system generates a $79.05-\mathrm{GHz}$ pulse with 0 - to $180 \mathrm{~V} / \mathrm{cm}$ amplitude and a variable width. The experiment is triggered at the $1-\mathrm{kHz}$ repetition rate of the frequencydoubled Nd:YLF laser used to pump the dye lasers. The linewidth of the laser responsible for the last transition is about $10 \mathrm{GHz}$. Amplified spontaneous emission of this laser drives the $3 p-3 d$ transition, while the output of the oscillator populates an $n f$ Rydberg state. The spontaneous emission can effectively drive the $3 p-3 d$ transition, but is not strong enough 

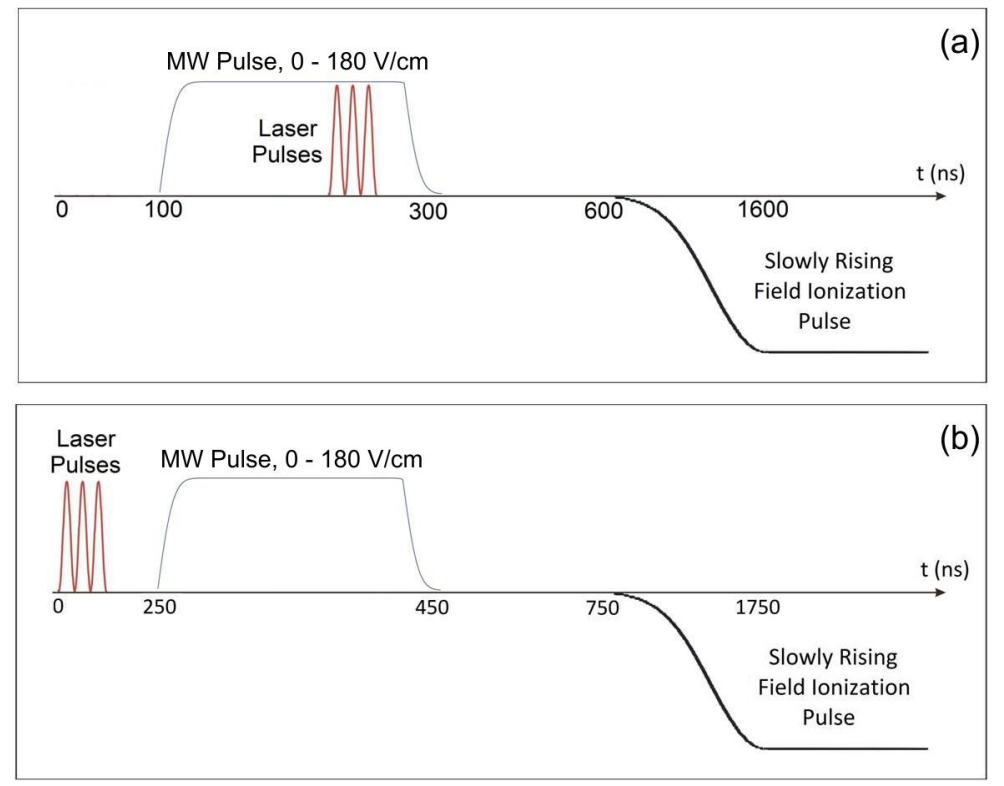

Figure 3.12: Experimental timing diagram. (a) Atoms are excited in the presence of the $79.05-\mathrm{GHz}$ microwave (MW) field. (b) Rydberg states created in laser excitation are subjected to the microwave pulse. About 300 ns after the microwave pulse, a slowly rising FIP ionizes atoms, and either electrons or ions are detected by the MCP, depending on the polarity of the FIP.

to excite an observable number of atoms to Rydberg states or to photoionize them. To populate the $3 d$ level with more atoms, we have also used a Toptica DL100 infrared diode laser to drive the transition from $3 p$. The laser beams are sent to the vacuum chamber, through 1-cm holes in the side plates surrounding the cavity, and are focused to less than 1-mm diameter spots to cross with the atomic beam. Unless stated otherwise, the laser field and microwave field are polarized vertically.

\subsubsection{Experimental Observations}

We start an investigation of ionization mechanisms at $80 \mathrm{GHz}$ by studying the total excitation spectrum in the case when the microwave field is on during the laser excitation as shown in Fig. 3.12 (a). It was demonstrated before that a spectrum of $\mathrm{H}$ atoms develops sidebands when exposed to 10-GHz microwave field [15]. Similarly, Pillet et al. showed a $15.4-\mathrm{GHz}$ sideband spectrum in radiative collisions in $\mathrm{Na}$ [20]. Later, in an attempt to connect field and photon viewpoints on ionization, excitation spectra of Na Rydberg states in $15-\mathrm{GHz}$ microwave field were recorded, yielding spectra in which an increasing number 


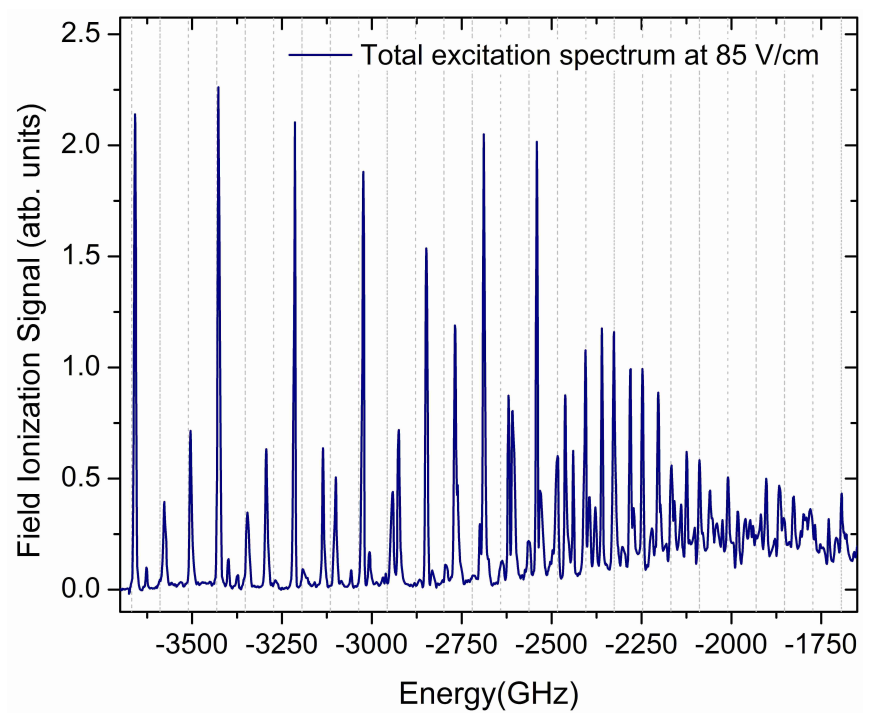

Figure 3.13: A total excitation spectrum of atoms excited in the presence of the $85-\mathrm{V} / \mathrm{cm} 80-\mathrm{GHz}$ microwave field. Ions are detected. Zero-field Rydberg states are partially ionized at this field, and sidebands emerge around them. The first large peak on the left corresponds to $n=30$. The FIP amplitude is about $800 \mathrm{~V} / \mathrm{cm}$, and it is capable of ionizing $n>25(-5260 \mathrm{GHz})$ states.

of sidebands developed about zero-field Rydberg states as the microwave field amplitude was increased [19].

Similarly, our observations indicate that zero-field states develop sidebands in $80-\mathrm{GHz}$ microwave field that can be populated if the microwave field is on during the excitation. The microwave pulse is on before the first laser, and excited atoms spend about $30 \mathrm{~ns}$ in the field before it is turned off with a 15-ns fall time. Fig. 3.13 shows a characteristic spectrum taken at a medium microwave field strength of $85 \mathrm{~V} / \mathrm{cm}$ and detecting ions. At this amplitude, lower Rydberg states are not significantly ionized, but extra peaks start to develop around them. For instance, two large peaks in Fig. 3.13, $n=30$ at -3655 $\mathrm{GHz}$ and $n=31$ at $-3420 \mathrm{GHz}$ have one sideband peak each, spaced by the microwave photon from the corresponding zero-state, on the right- and left-hand side, at -3576 and $-3500 \mathrm{GHz}$, respectively. Higher $n$ states develop more sideband peaks at this microwave field amplitude, and the spectrum is less easy to interpret in this regime. In accord with the work of van Linden van den Heuvell et al. [19], our results indicate that the dominant effect of the microwave field is to convert the zero-field Rydberg state into a carrier and sidebands, the number of which increases with the microwave field. To clarify, Fig. 3.14 

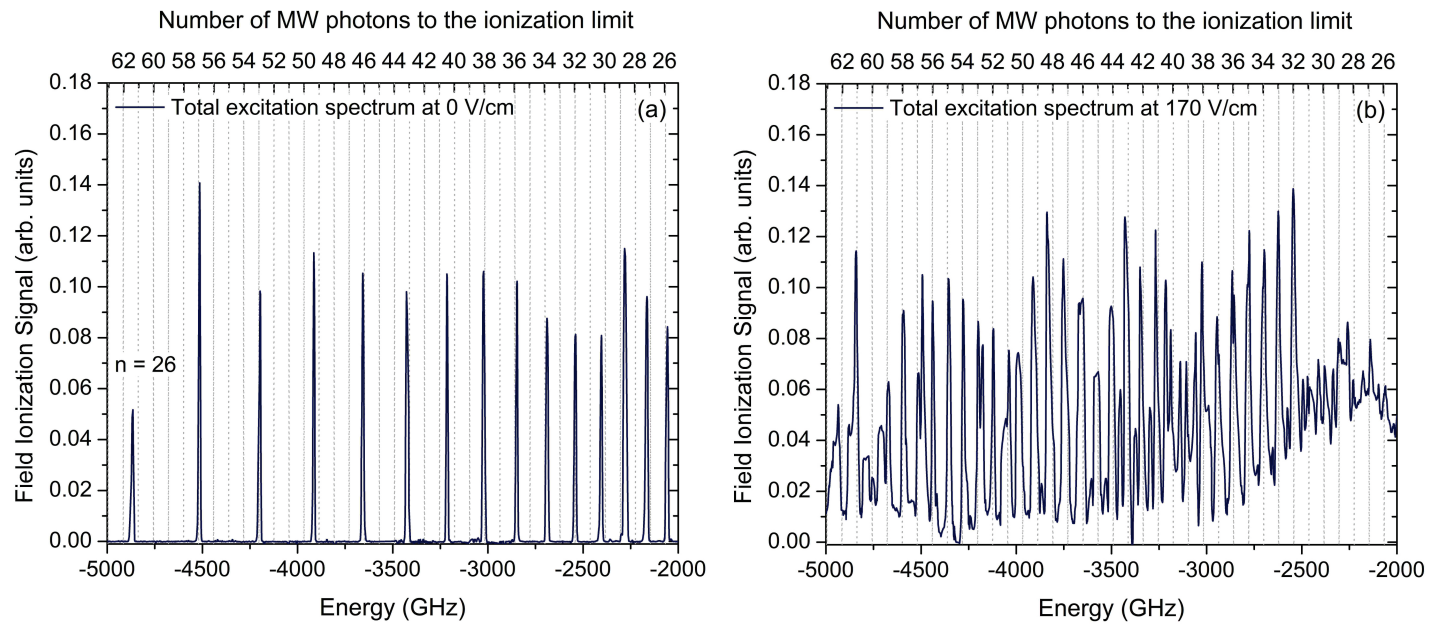

Figure 3.14: Field ionization signal as a function of the laser frequency, in terms of energy relative to the ionization limit (IL). (a) A zero-field spectrum of Rydberg states in the range of $26 \leq n \leq 40$. We detect only $f$ states due to vanishingly small oscillator strength of the transition to an $n p$ state. (b) A total excitation spectrum of atoms excited in the presence of $170-\mathrm{V} / \mathrm{cm} 80-\mathrm{GHz}$ microwave field. Ions are detected. The spectrum exhibits extra peaks between Rydberg states due to emerging sidebands of zero-field states. Sidebands are an integral number of microwave photons from the corresponding zero-field Rydberg state. Sidebands of different peaks overlap producing an irregular structure of peaks.

shows a comparison of spectra obtained by scanning the frequency of the last laser when the microwave field is off (a) or is $170 \mathrm{~V} / \mathrm{cm}$ (b). Again, ions were detected in both cases, and we observe well-resolved peaks between $n=26$ and 45 Rydberg states in Fig. 3.14 (a). When the microwave field is on, the excitation spectrum is altered significantly from the one shown in Fig. 3.14 (a). We also do not see very pronounced Rydberg peaks as in Fig. 3.13. At this microwave field, the amplitude of the carrier, the zero-field Rydberg state, is no longer larger than that of its sidebands. The spectrum of Fig. 3.14 (b) is quite illegible and represents a situation in which each zero-field Rydberg state has several sidebands above and below it, and the sideband manifolds of adjacent Rydberg states overlap. Direct comparison of spectra taken at the $85-\mathrm{V} / \mathrm{cm}$ (Fig. 3.13), 135-V/cm (discussed below), 170-V/cm (Fig. 3.14 (b)), and various other microwave field amplitudes showed that sideband and Rydberg peaks do not experience energy shifts and remain at the same frequencies as the amplitude is increased. As was discussed in Chapter 1, van Linden van den Heuvell et al. showed that when the microwave field exceeds the Inglis-Teller limit, sideband peaks are pulled together and form a regular set of peaks spaced by $15-\mathrm{GHz}$ [19]. At this field, the sideband 


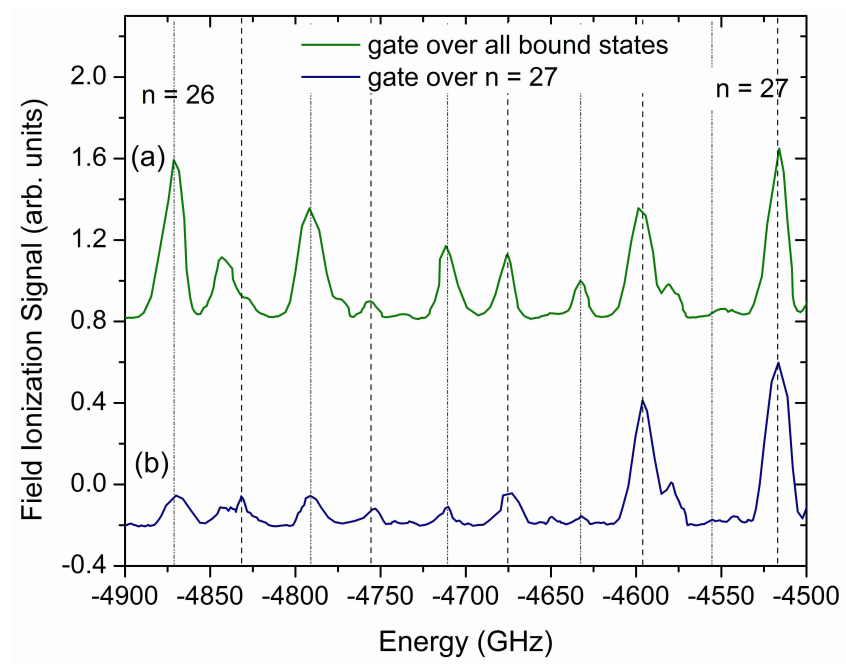

Figure 3.15: Field ionization signal as a function of laser frequency between the $n=26$ and 27 sates. The bound population is detected (electrons) after the laser excitation in the presence of the $135-\mathrm{V} / \mathrm{cm}$ microwave field. (a) The integration gate is set to record signal from all bound states. (b) The integration gate is set to record signal only from $n=27$. Vertical dash-dot-dotted and dashed lines are $79 \mathrm{GHz}$ apart from $n=26$ and 27 peaks, respectively. Three sideband peaks are observed for each of these states, and their respective sideband manifolds overlap. Nevertheless, atoms excited at sidebands of $n=27$ stay in $n=27$ : no population transfer between 26 and 27 Rydberg states is observed.

sets, as well as the Stark manifolds, of adjacent Rydberg states overlap, and a transition from $n$ to $n+1$ Rydberg states occurs which results in a significantly lowered ionization field of $n$ Rydberg state. Thus, when a continuous set of sideband peaks is formed, atoms initially tuned to a low Rydberg state might be driven to the vicinity of the continuum by a sequence of $n \rightarrow n+1$ transitions. However, we do not observe a regular structure of peaks spaced by a microwave photon from each other: sideband peaks are always an integral number of microwave photons from a corresponding zero-field Rydberg state, different state for different sideband peaks. Also, to investigate if the observed spectrum contains very high Rydberg states and is related to the ionization limit, we repeated the measurement of Fig. 3.14 (b) with $1-\mathrm{V} / \mathrm{cm}$ static field. The resulting total excitation spectrum turned out to be identical to the one recorded before, with the only difference that sidebands became slightly broader. That implies that the majority of atoms is still in the low states for which such an increase in static field does not introduce any observable dc Stark shift.

It is possible to generate a small number of sideband peaks at a microwave field that is not strong enough to ionize a population in these sideband peaks. Thus, it is possible 


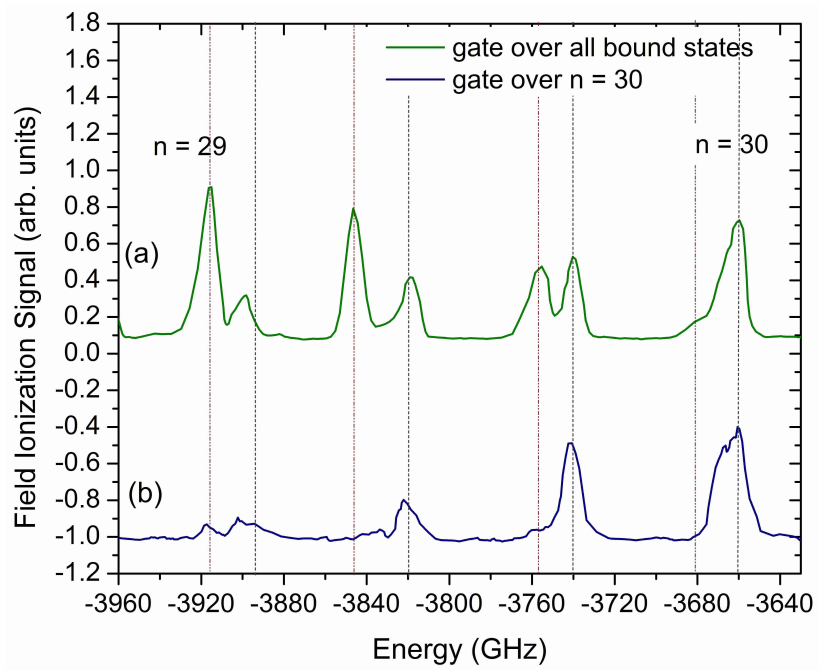

Figure 3.16: Field ionization signal as a function of laser frequency between the $n=29$ and 30 states. The bound population is detected (electrons) after the laser excitation in the presence of $135-\mathrm{V} / \mathrm{cm}$ microwave field. (a) The integration gate is set to record signal from all bound states. (b) The integration gate is set to record signal only from $n=30$. Vertical dash-dot-dotted and dashed lines are $79 \mathrm{GHz}$ apart from $n=29$ and 30 peaks, respectively. Two pronounced sideband peaks are observed for each of these states, as their respective sideband manifolds overlap. Atoms excited at sidebands of $n=30$ stay in $n=30$ : no population transfer between 29 and 30 Rydberg states is observed.

to find a regime when the population is still bound and, using electron detection, learn what zero-field Rydberg state it belongs to. Using the negative FIP of about $800 \mathrm{~V} / \mathrm{cm}$ allows us to ionize lower Rydberg states and time-resolve signal coming from different states. Fig. 3.15 (a) shows a part of a spectrum, between $n=26$ and 27 Rydberg states, of atoms excited in the presence of the $135-\mathrm{V} / \mathrm{cm}$ microwave field. We used a wide integration gate to detect signal from all bound states and observed three pronounced peaks spaced 79, 158, and $237 \mathrm{GHz}$ from $n=26$ (vertical dashed-dot-dotted lines) and three peaks on the left of $n=27$, also spaced 1, 2, and 3 microwave photons from that state (vertical dashed lines). The observed spectrum also shows another peak at $-4840 \mathrm{GHz}$ that is due to a microwave mixing and excitation of the $27 p$ state. It is clear that, at this microwave field strength, sideband manifolds of two Rydberg states overlap (the second sideband peak of $n=27$ is between the second and the third sideband peaks of $n=26$ ) and, in order to figure out if there is any population transfer from 26 to 27, we repeat the measurement recording only the signal from $n=27$. Fig. 3.15 (b) shows that a significant signal is observed only from $n=27$ Rydberg state and from peaks that are separated an integral number of 
microwave photons from it. It is impossible to completely exclude the $n=26$ signal from the integration gate, however, it is obvious that the leaking signal is very small, as even the forth sideband peak of $n=27$ in now apparent. Overall, even though the manifolds of two Rydberg states overlap, there is no significant transition of population from $n$ to $n+1$, which becomes even clearer in Fig. 3.16. Fig. 3.16 (a) shows a part of spectrum between $n=29$ and 30 when the integration gate allows signal from all bound states, while the spectrum of Fig. 3.16 (b) was recorded with the integration gate over $n=30$. As it is easier to discern these two states in time, less signal from $n=29$ is collected when the gate is set over the $n=30$ signal. Again, it is evident that atoms excited at frequencies an integral number of microwave photons from a zero-field Rydberg state are, in fact, excited to that Rydberg state, and we do not observe any population transfer to $n+1$ state when manifolds overlap, a result different from earlier observations at $15 \mathrm{GHz}$, nor do we observe ionization at $E=1 / 3 n^{5}$. In sum, there are two striking differences from the $15-\mathrm{GHz}$ measurements: overlapping sideband sets do not form a regular structure of peaks spaced by $80 \mathrm{GHz}$, and no $n \rightarrow n+1$ transition occurs that could lower the ionization fields.

To further investigate ionization at $80 \mathrm{GHz}$, we measured ionization thresholds of Rydberg states. For each state, the laser frequency was fixed, and atoms were excited in zero-field to be later ionized by a 500-ns microwave pulse. To account for the presence of the high-lying states, a survival probability as a function of the microwave field amplitude was measured. Figs. 3.17 (a) and (b) show what fraction of atoms was ionized by the microwave field for the two characteristic cases of $n=36$ and 38. First, a vertically or horizontally polarized laser excited $|m|=0$ and 1 states and $|m|=0,1$, and 2 states, respectively, in the presence of $10-\mathrm{mV} / \mathrm{cm}$ static field. It is evident that ionization curves are similar for both laser polarizations, and 50\% ionization thresholds are very different from the two limiting cases observed in 15-GHz ionization experiments [13]: a dashed line signifies a $1 / 3 n^{5}$ threshold, and a dotted line the $1 / 9 n^{4}$ hydrogenic ionization field. However, when a static field of $4.5 \mathrm{~V} / \mathrm{cm}$ is added, the ionization threshold changes substantially for $n=36$ but not for $n=38$. A not immediately obvious difference between these states comes from the fact that the spacing between $n=36$ and adjacent states is different from the spacing 

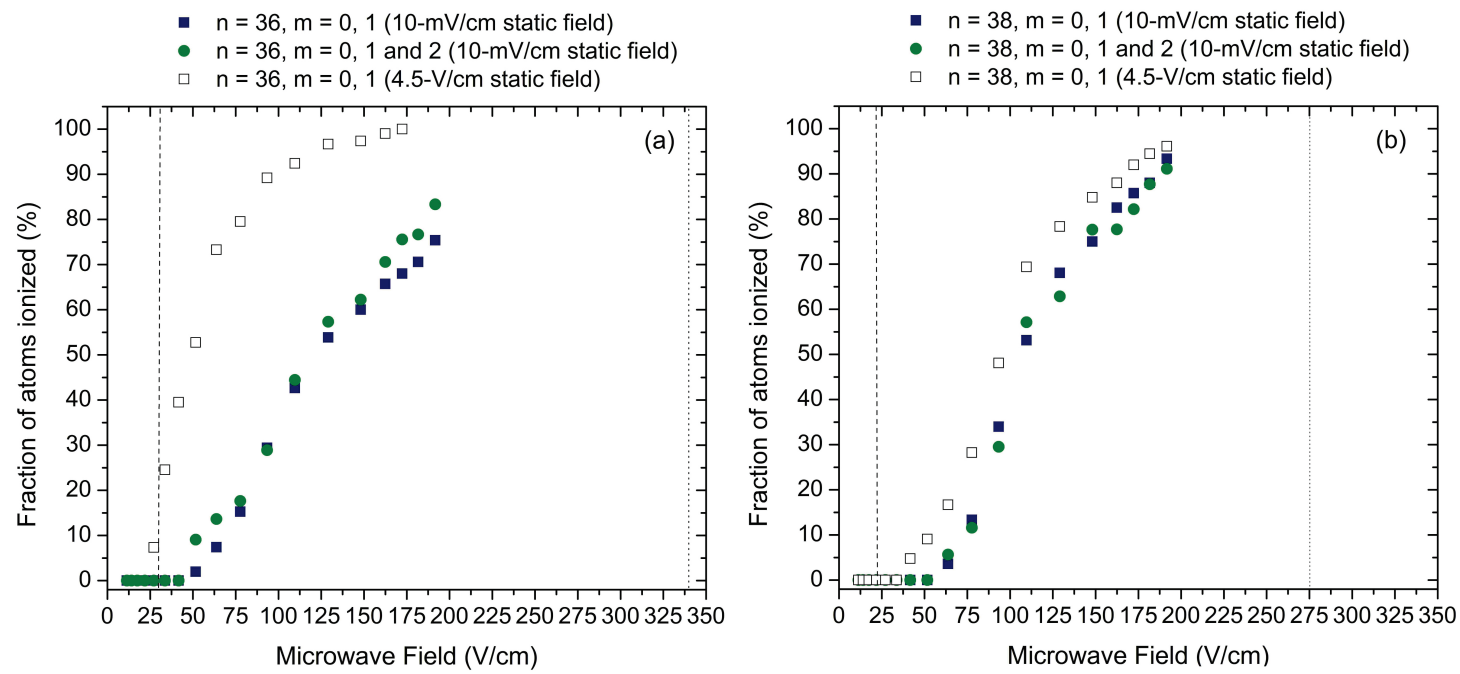

Figure 3.17: A fraction of atoms ionized as a function of microwave field amplitude when the laser is tuned to (a) $n=36$ and (b) $n=38$. Electrons are detected, and a percentage of population left bound after the microwave pulse is subtracted from $100 \%$. Laser polarization is parallel (squares) and perpendicular (circles) to the microwave polarization. The data are taken in the presence of small (filled figures) and large $4.5-\mathrm{V} / \mathrm{cm}$ static field (open figures). Ionization thresholds observed at $15-\mathrm{GHz}$ ionization of $\mathrm{Na}$ are shown by a dotted vertical line (Inglis-Teller limit of $1 / 3 n^{5}$ ) and a dashed vertical line (field ionization at $1 / 9 n^{4}$ ). The ionization field of $n=36$ decreases substantially with the static field due to an enhanced probability of making a transition to higher Rydberg states, while ionization of $n=38$ does not depend significantly on the static field because the resonance conditions are not satisfied due to the spacing between $n=38$ and higher Rydberg states.

between 38 and its neighbors. Namely, the spacing between 36 and 37 is $1.78 w$, where $w=79 \mathrm{GHz}$, and $6.11 w$ between 36 and 40 . So, 37 and 40 are the closest to a multiphoton resonance states that $n=36$ has. However, these are not the exact resonances, so only a very tiny fraction of population can be driven to higher states and be ionized at smaller fields. Nevertheless, if a $4.5-\mathrm{V} / \mathrm{cm}$ static field is added, the resonances become broader, and a multiphoton transition is more probable. Indeed, we measured the final state distribution in the presence of very small and large static fields: in the former case the population stays at $n=36$, as the peak becomes smaller due to an increasing microwave field, while in the latter case, atoms remain in the initial state along with populating $n=37$ and 40 . Consequently, we attribute the difference in ionization curves of Fig. 3.17 (a) to the fact that multiphoton resonant transitions to higher states become possible for $n=36$ when the large static field is present. However, a similar behavior is not observed in Fig. 3.17 (b) for $n=38$ because the next state is $1.55 \mathrm{w}$ away from it, and $4.5-\mathrm{V} / \mathrm{cm}$ static field is not 


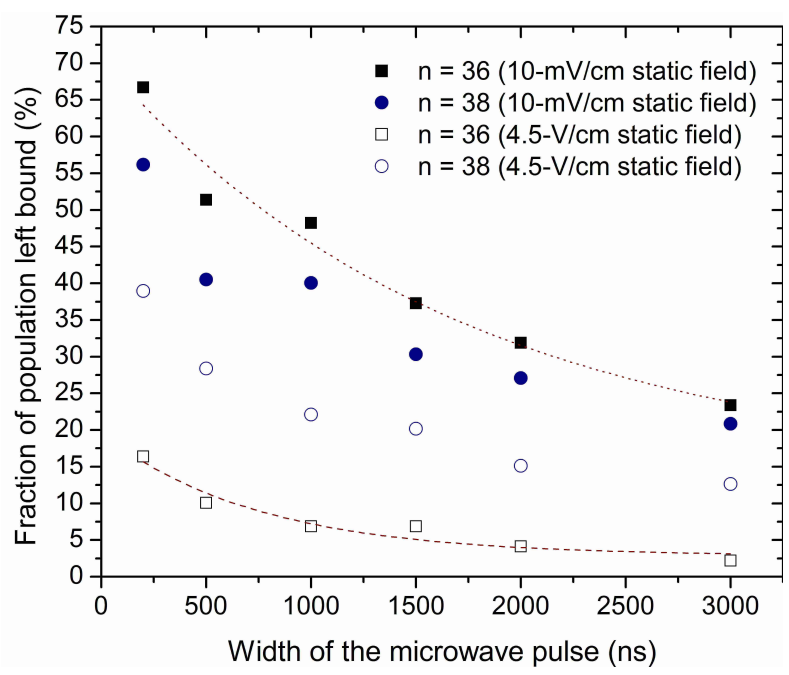

Figure 3.18: A fraction of population left in bound states after the $120-\mathrm{V} / \mathrm{cm}$ microwave pulse of varied width. Atoms are excited to $n=36$ (filled squares) and $n=38$ (filled circles) in the presence of a small $10-\mathrm{mV} / \mathrm{cm}$ and large $4.5-\mathrm{V} / \mathrm{cm}$ static field (open squares and circles respectively). Data points of $n=36$ are fitted with exponential decay curve of first order as $A e^{-x / t}+B$. A curve taken with $4.5-\mathrm{V} / \mathrm{cm}$ static field decays twice as fast as a curve taken with a small static field (740 and $1700 \mathrm{~ns}$ respectively). In contrast to the ionization experiment at $15 \mathrm{GHz}$, ionization fields depend strongly on the width of the pulse.

large enough to bring $n=38$ in resonance with it. Indeed, the final state distribution after the microwave pulse does not yield any population transfer to higher states except for a small peak that belongs to $n=41$ (the spacing between the 38 and 41 states is 4 microwave photons), which is not altered in the case of small or large static field. Overall, it seems that ionization at $80 \mathrm{GHz}$ is enhanced only via resonant transitions to higher lying states. It is a regime, then, different from the one observed below $20 \mathrm{GHz}$, and, in contrast to previous findings [13], there is a strong dependence of ionization fields on the width of the pulse. Fig. 3.18 shows characteristic population decay curves as a function of the width of the $120-\mathrm{V} / \mathrm{cm}$ microwave pulse when the laser is tuned to $n=36$ (filled squares) and 38 (filled circles) in $10-\mathrm{mV} / \mathrm{cm}$ and $4.5-\mathrm{V} / \mathrm{cm}$ static field (open squares and circles respectively). We observe that, even for very long pulses, decay curves are not saturated, and ionization fields decrease if atoms are subjected to a larger number of cycles of the microwave field.

In Fig. 3.19 we show microwave fields required to ionize $50 \%$ and $10 \%$ of atoms versus binding energy. These data are extracted from ionization curves, similar to the ones shown in Fig. 3.17, that are obtained by fixing the last laser frequency at a Rydberg state $(1 / 5 \leq$ $\Omega<\infty)$ and changing the amplitude of the 500-ns microwave pulse. The observed thresholds 


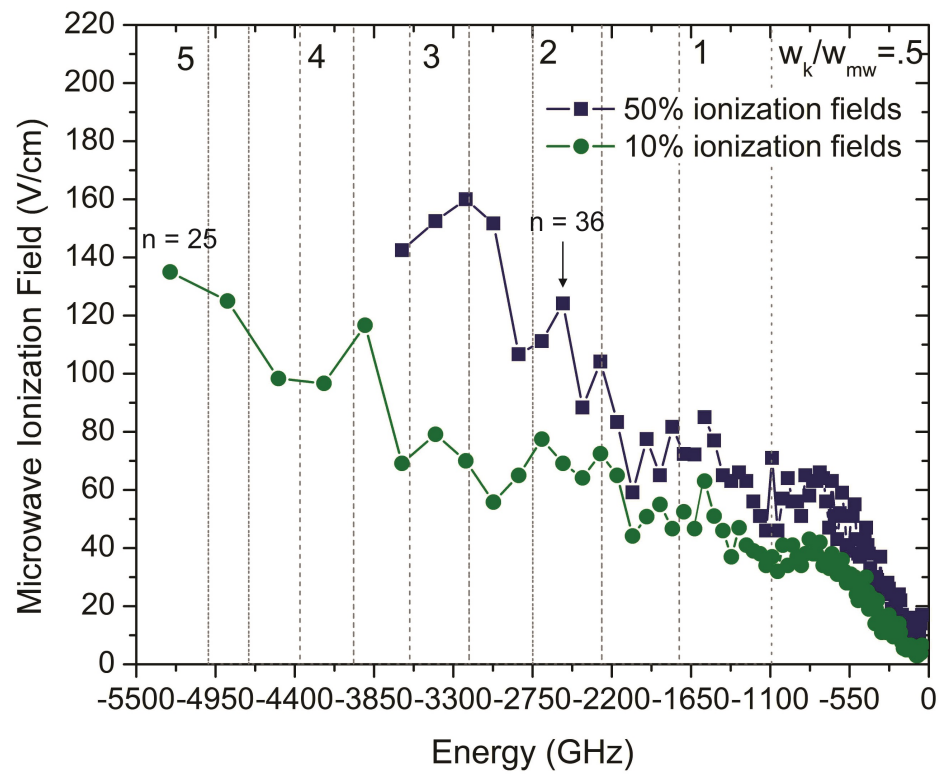

Figure 3.19: Microwave fields as a function of laser tuning for $50 \%$ and $10 \%$ ionization. Microwave pulse width is $500 \mathrm{~ns}$. Vertical dashed lines on the graph correspond to the energies of Rydberg states for which the classical Kepler frequency equals $5 w-0.5 w$.

are significantly higher than the $1 / 3 n^{5}$ fields reported for experiments below $20 \mathrm{GHz}$. They also do not exhibit a monotonic decrease, but yield a certain structure reminiscent of that observed in the 38-GHz Li ionization experiment [57]. A structure observed for low Rydberg states $(\Omega<1)$ is not related to the ionization limit, but is determined by a spacing between Rydberg states. For example, the $10 \%$ ionization fields for $n=27(-4513 \mathrm{GHz})$ and 28 are noticeably smaller than the ionization field of $n=29$. The spacings between $n=27$, 28 , and 30 are $4.01 w$ and $10.95 w$ respectively, while $n=29$ is not resonant with any higher Rydberg states. Fig. 3.20 shows an expanded view of the $50 \%$ ionization fields near the limit, where $\Omega>1$. Similar to the $38-\mathrm{GHz}$ results, we see a structure of peaks spaced by an integral number of microwave photons from the first peak below the ionization limit. Jensen et al. [25] predicted that, at these binding energies, atoms ionize through a sequence of transitions to excited states which results in a field independent of the principle quantum number: $E=2.4 \omega^{5 / 3}$. A corresponding value is marked by a dotted red line in Fig. 3.20 and is higher than the observed $50 \%$ ionization fields. Not accounting for the structure, our data are in agreement with the prediction of independence of ionization thresholds on binding 


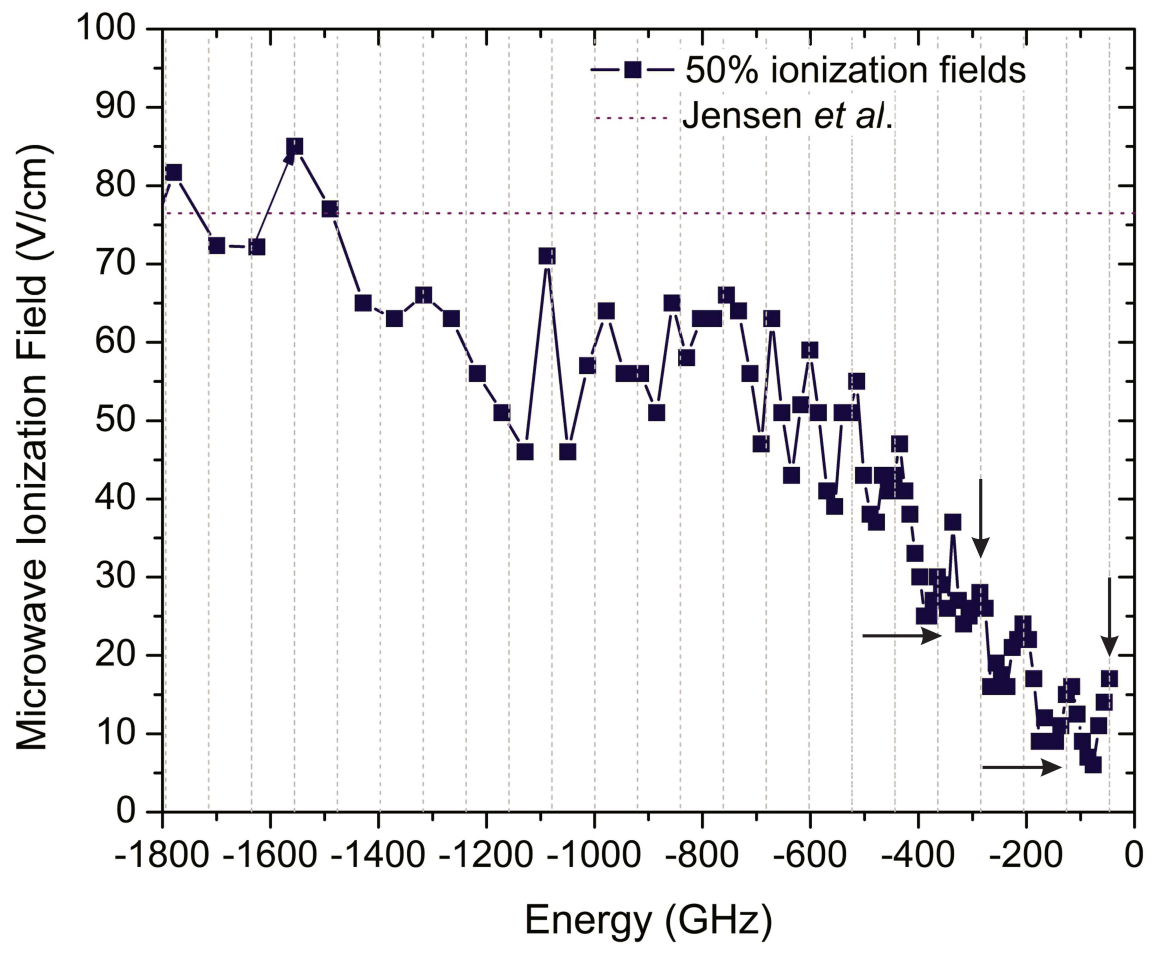

Figure 3.20: An expanded view of the $50 \%$ ionization fields in the $\Omega>1$ regime. Up to $-500 \mathrm{GHz}$, our result is in agreement with a prediction of Jensen et al. (Eq. 1.16) [25] (red dotted line) that the average ionization field does not depend on $n$. That prediction, however, is not intended to reproduce the structure we observe. As shown by the vertical dashed lines spaced by $79 \mathrm{GHz}$, the structure in the ionization fields is at the microwave frequency. Vertical arrows show the first $(W \sim 0.5 \omega)$ and the fourth maximum $(W \sim 3.5 \omega)$ of the structure below the IL. The horizontal lines show the first $(W \sim \omega)$ and the forth minima $(W \sim 4 \omega)$.

energy up to $-500 \mathrm{GHz}$, or 6 microwave photons from the ionization limit. At this point, the ionization field drops drastically which we attribute to photoionization of atoms. It is important to emphasize here that the threshold fields shown in Fig. 3.19, in fact, depend on the width of the pulse and in itself do not hold a great value. However, an important conclusion can be drawn from it. In contrast to the results of earlier ionization experiments, in the $\Omega<1$ region, threshold fields do not fall monotonically with the principle quantum number. Near the limit, where $\Omega>1$, a structure of peaks spaced by the microwave photon is observed. Similar structures were observed at the 17 and $38-\mathrm{GHz}$ ionization experiments. The structures that we observe in these two regions, however, are different: at low Rydberg states it is governed by the spacing between Rydberg states, while at energies where $\Omega>1$ peaks are displaced from the ionization limit. Further, we measured single photon ionization 


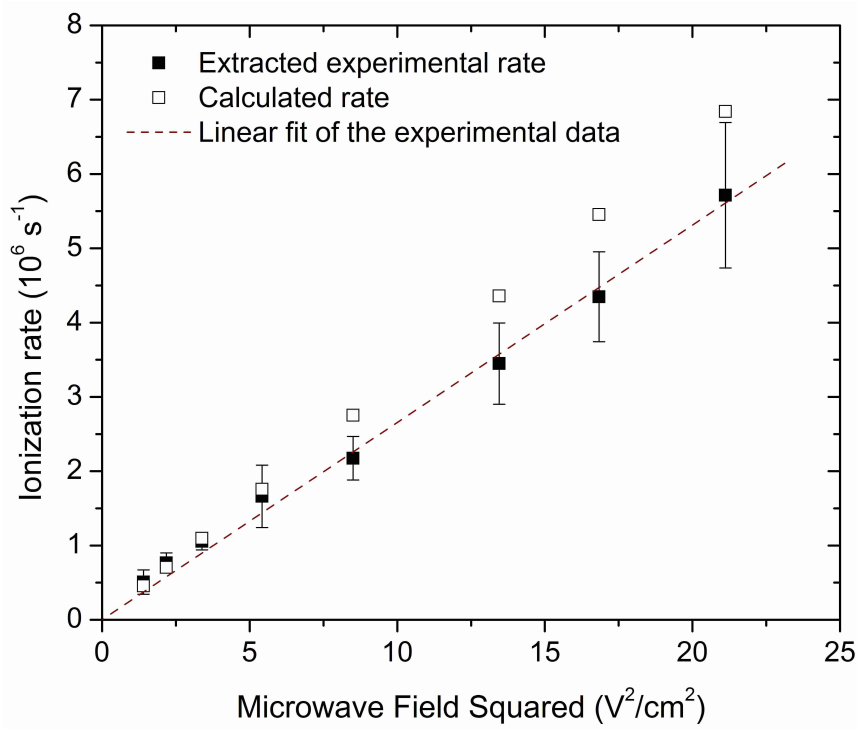

Figure 3.21: Extracted single photoionization rates compared to the Fermi's Golden Rule predicted rates when the laser is tuned $80 \mathrm{GHz}$ below the IL as shown by the horizontal arrow in Fig. 3.20. The experimental data points are fit with $A x$. The observed rates are linear in power and are in a good agreement with the predicted values.

rates of the $80-\mathrm{GHz}$ microwave field. We tuned the laser frequency one microwave photon below the ionization limit, to the first minimum of the structure of Fig. 3.20 shown by the horizontal arrow. We varied the pulse width measuring the fraction of population left bound after the pulse for various pulse amplitudes. For each value of the field, data points were fit to an exponential function $A e^{-x / t}$, a decay time $t$ was measured, and photoionization rates were extracted. Fig. 3.21 shows that the observed photoionization rates are, as expected, linear in power and are in a good agreement with rates predicted by the Fermi's Golden Rule [52]:

$$
\Gamma=2 \pi|<f| r E_{w}|i>|^{2}
$$

where $E_{w}=0.5 E$ due to a rotating wave approximation. The final state is above the ionization limit, consequently, we have to consider a bound-continuum radial matrix element that is given by Delone et al. [26]:

$$
<\epsilon|r| i>=\frac{0.4108}{n^{3 / 2} w^{5 / 3}}
$$


The angular part of the matrix element is given by

$$
<\operatorname{lm}|\cos \theta| l+1 m>=\sqrt{\frac{(l+1)^{2}-m^{2}}{(2 l+3)(2 l+1)}} .
$$

In our experiment, $l=3$ and $|m|=0$.

To elucidate if atoms ionize through photoionization in the region above $-500 \mathrm{GHz}$, where ionization fields drop down fast, we tuned the laser frequency four microwave photons below the ionization limit, as marked by the left horizontal arrow in Fig. 3.20, and observed atoms photoionize through a sequence of microwave transitions to the continuum. To observe atoms making these transitions, we narrowed the microwave pulse to $30 \mathrm{~ns}$ and studied the final state distribution after the pulse. The resulting oscilloscope traces are shown in Fig. 3.22. The field ionization signal appears on the oscilloscope screen later if atoms are more deeply bound.

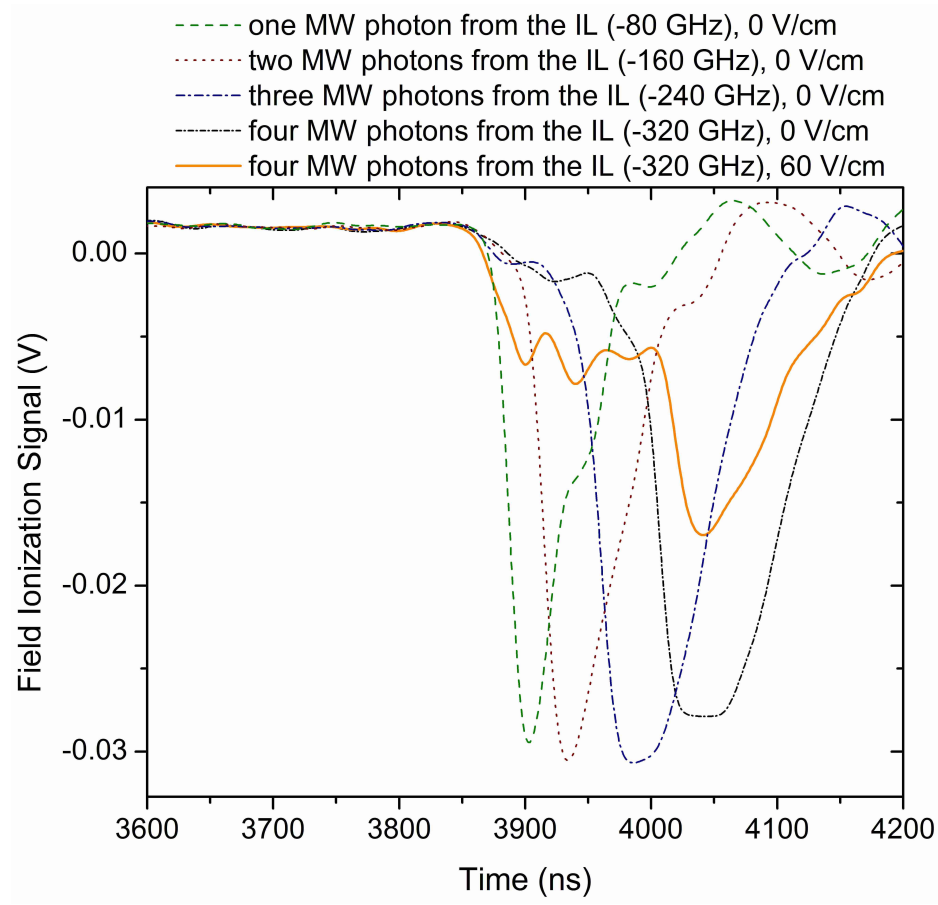

Figure 3.22: An oscilloscope trace taken when the laser is tuned one, two, three, four MW photons from the IL with the microwave field off (broken lines), and when atoms are excited $4 \mathrm{MW}$ photons from the IL and exposed to $60-\mathrm{V} / \mathrm{cm} 30$-ns microwave pulse (solid line). The microwave field populates states three, two, and one photons below the IL where atoms photoionize.

Four large peaks of Fig. 3.22 were obtained when the laser was tuned one microwave 
photon (dashed line) from the ionization limit, two microwave photons (dotted line), three microwave photons (dash-dotted line), and four microwave photons (short-dotted line) with the microwave field off. An orange solid curve shows a trace taken when the laser frequency was set four microwave photons from the ionization limit, and the short 30-ns microwave pulse was on after the excitation. It is evident that some fraction of the population is left in the initial state, but states one, two, and three microwave photons above are populated at the same time as well. If the width of the pulse is increased, more atoms are driven to the states one or two photons from the ionization limit where they can photoionize rapidly. We, however, do not observe any population transfer to lower Rydberg states.

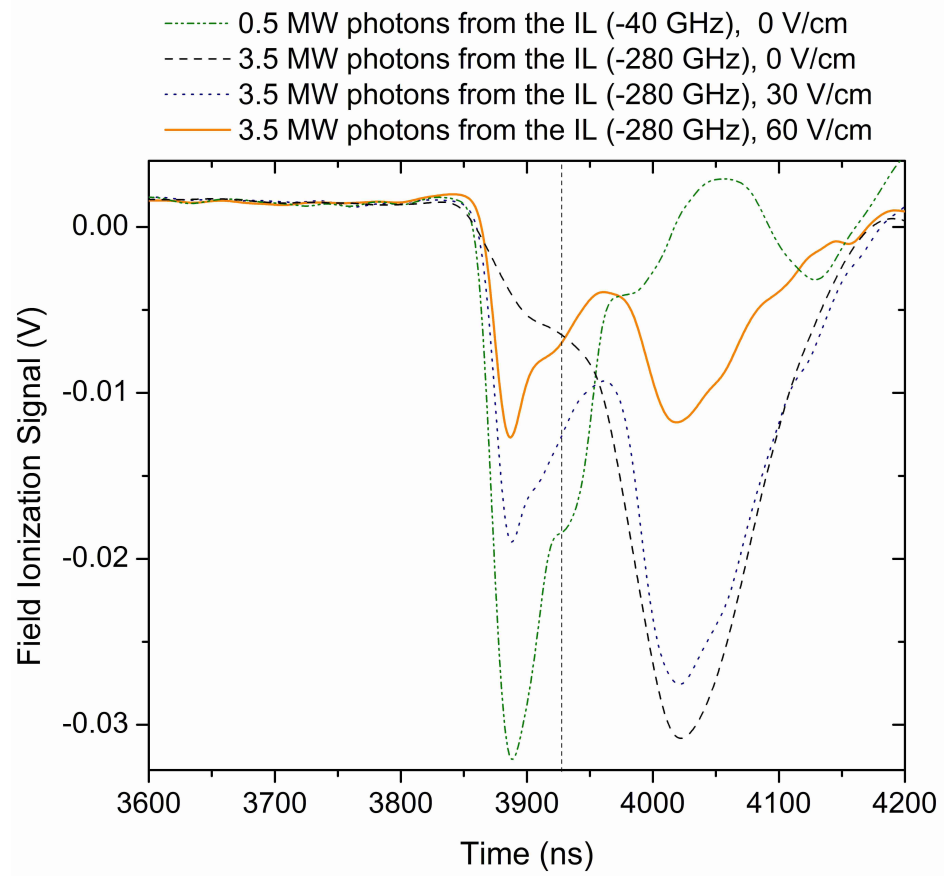

Figure 3.23: An oscilloscope trace taken when the laser is tuned half a MW photon from the IL with the microwave field off (dash-dotted line) and $3 \mathrm{MW}$ photons below it with the microwave field off (dashed line), after $30 \mathrm{~V} / \mathrm{cm} 30$-ns pulse (dotted line), and after $60 \mathrm{~V} / \mathrm{cm} 30$-ns pulse (solid line). Both energies are marked by vertical arrows in Fig. 3.20. The microwave field does not populate states between the initial state and states half a photon below the IL. Rather a large fraction of population is found in the extremely high-lying states where atoms can survive long and large amplitude microwave pulses. The vertical dashed line shows the position of the peak corresponding to the zero-field states tuned 1.5 microwave photons below the IL. Bumps on the falling edge of the peaks are due to the electrical ringing, and do not correspond to the signal from atoms excited 1.5 or 2.5 photons below the limit.

Our previous investigations at 17 and $38 \mathrm{GHz}$ showed that population can be trapped in the extremely high-lying states after even a very long microwave pulse. However, when the 
laser is tuned to minima of the structure of Fig. 3.20 atoms tend to photoionize, as discussed above, and no trapping in the high-lying states is observed. Nevertheless, our results suggest that the high-lying state production occurs when the laser is tuned to maxima of the structure of Fig. 3.20, or an integral number of microwave photons from the first peaks of that structure. Indeed, Fig. 3.23 shows oscilloscope traces when the laser is tuned to the fourth peak (3.5 microwave photons below the ionization limit, $-280 \mathrm{GHz}$ ) with the microwave field off (dashed line), after the 30-ns 30-V/cm microwave pulse (dotted line), after the 30-ns $60-\mathrm{V} / \mathrm{cm}$ microwave pulse (solid line), and when the laser is tuned to the extremely high-lying states within $40 \mathrm{GHz}$ of the ionization limit with the microwave field off (dash-dotted line). Energies corresponding to these states are shown in Fig. 3.20 by vertical arrows. It is clear that, first, there is no population transfer to states one or two microwave photons from the initial state for any pulse widths we were able to try. Second, there is a substantial number of atoms found in the extremely high-lying states when the initial state only starts being ionized and when it is ionized significantly. Consequently, the population found in the high-lying states is depleted less rapidly in the strong microwave field than that in the initial state. Moreover, in contrast to atoms excited one microwave photon from the ionization limit that photoionize rapidly, the population in the high-lying states within half a photon survives even very long microwave pulses of large amplitudes.

Our observations suggest that, in the $\Omega<1$ regime, even when manifolds of sidebands overlap, first, sideband peaks do not coalesce to form a regular structure of peaks that are spaced exactly a microwave photon from each other as was seen at $15 \mathrm{GHz}$ [19]: at any microwave field strength we tried, sideband peaks remained at the same energies, namely, spaced an integer number of photons from the corresponding zero-field Rydberg state. Second, in contrast to previous experiments, no population transfer from $n$ to $n+1$ states was observed, even though, for example, sideband manifolds of $n=26$ and 27 and $n=29$ and 30 overlapped. An obvious difference between microwave experiments at 15 and $80 \mathrm{GHz}$ is the size of the microwave photon. Sodium does not have large quantum defects, 1.35 and 0.85 being the only quantum defects larger than 0.015. Thus, a coupling $\omega_{0}$ between extreme Stark states of adjacent manifolds is small, and the detuning $\Delta$ between them, which is on 
the order of the microwave frequency $\omega$ in the situation when sidebands overlap at $15 \mathrm{GHz}$, is significantly larger than the couplings at $80 \mathrm{GHz}$. In previous experiments is was shown that, when the detuning is comparable with the size of a coupling, $\Delta \approx \omega_{0}$, the $n \rightarrow n+1$ transition occurs lowering the ionization field thresholds $[13,19]$. On the other hand, when this condition is not satisfied, $|m|=2 \mathrm{Na}$ states [13] and Li atoms in small static fields [18] ionize by field ionization at $1 / 9 n^{4}$. Another obvious example of such a situation is an atom of $\mathrm{H}$ where levels are not coupled. It was shown that ionization fields are lowered only when an occasional resonance between adjacent states occurs in case when the spacing equals an integer number of the field photons [21]. Thus, it is not entirely surprising that atoms of $\mathrm{Na}$ at $80 \mathrm{GHz}$, for which $\omega>>\omega_{0}$, do not show ionization at the Inglis-Teller limit of $1 / 3 n^{5}$. We observe higher fields with a definitive structure modified by resonances between Rydberg states when $\Omega<1$. However, there are only a few states for which the $\Delta n$ spacing equals an integer number of microwave photons. A large static field, though, can lower ionization fields of some states enhancing and broadening the resonances. For example, a fraction of atoms excited to $n=36$ is observed to make a transition to $n=37$ only when the static field is increased, as the spacing between these states is not exactly two photons. As a consequence, the ionization field of this state is significantly lowered when the $4.5-\mathrm{V} / \mathrm{cm}$ static field is present; that does not occur for $n=38$ for which the resonant condition to make a transition to $n=39$ is not satisfied even at that field.

Another important difference from earlier observations is the dependence of fraction of atoms ionized on the width of the pulse. It was discussed by van Linden van den Heuvel et al. that, when $\Delta \approx \omega_{0}, n$ to $n+1$ transition has a substantial probability to happen in first few cycles [19], and, Pillet et al. showed that the 15-GHz ionization fields of $s$ states of $\mathrm{Na}$ are independent of the microwave pulse width from 0.2 to $1 \mu \mathrm{s}$ [13], as the transition probability quickly saturates with the number of cycles. However, our results indicate a strong dependence of ionization fields on the number of cycles in the pulse at $80 \mathrm{GHz}$, which signifies that the ionization regime is different from the one observed at frequencies below $20 \mathrm{GHz}$. 


\subsection{Conclusions}

We studied ionization of Rydberg atoms by 17,38 , and $80-\mathrm{GHz}$ microwave fields for a broad range of initial states. The main result of this investigation is an observation of a substantial number of atoms left bound in very high-lying states and a significant effect of relatively small static fields on ionization processes.

Ionization of Rydberg atoms below $20 \mathrm{GHz}$ was studied substantially in many previous experiments $[13,19,18,55]$. It was shown that $|m|=0$ and 1 states of $\mathrm{Na}$ ionize at a field corresponding to the Inglis-Teller limit, $E=1 / 3 n^{5}$, and $|m|=2$ states ionize by hydrogenic field ionization at $E=1 / 9 n^{4}$. Results of the experiment that we conducted at $17 \mathrm{GHz}$ in the presence of very low static field showed a significant disagreement with previous works. First, apparently, only $|m|=0$ Rydberg states of Na ionize through a sequence of transitions to higher states at $E=1 / 3 n^{5}$. However, as the static field is increased, $|m|=1$ states also start ionizing at this field due to a splitting of degenerate sidebands into a quasicontinuum of states that allows transitions to higher $n$ states. In fact, our results match the observations of Pillet et al. [13] when $\sim 1.5-\mathrm{V} / \mathrm{cm}$ static field is present during the experiment. That implies that a static field of this order of magnitude was present in the previous experiments conducted in waveguides. That, obviously, precluded the possibility of observing atoms trapped in the high-lying states that are ionized by small static fields of tens of $\mathrm{mV} / \mathrm{cm}$ and are observed in our experiment.

The results of the experiment at a higher $38-\mathrm{GHz}$ microwave field in the region $\Omega<1$ indicate that ionization fields decrease with principle quantum number but not monotonically as in the experiments below $20 \mathrm{GHz}$. We observed a structure in ionization fields determined by the ratio of the spacing between Rydberg states to the microwave frequency. The $50 \%$ ionization thresholds also turned out to be higher than ones observed at $15 \mathrm{GHz}$ meaning that, at this frequency, we start to enter an intermediate regime where a multiphotoninduced ionization picture breaks due to the fact that the couplings between extreme Stark states are smaller than the field frequency. Moreover, the experiment conducted at $80 \mathrm{GHz}$ for $\mathrm{Na}$ atoms reinforces this point. The observed structure below $\Omega=1$ is more pronounced 
and is due to the spacing between zero-field Rydberg states. Our results suggest that, in a small static field, atoms can make a transition to a higher Rydberg state and be ionized at a lower field only if the spacing between these states nearly equals an integral number of microwave photons. Thus, in contrast to ionization at $17 \mathrm{GHz}$, some Rydberg states ionize at lower fields than a state with a higher principle quantum number. However, the static field can enhance ionization by broadening the resonances. Also, due to the large size of the microwave photon, a transition probability in one cycle is very small, so the observed ionization fields depend significantly on the number of cycles even for pulses longer than a microsecond.

In the region $\Omega>1$ our results at 17,38 , and $80 \mathrm{GHz}$ qualitatively agree with the prediction of Jensen et al. [25] that on average ionization fields do not depend on the initial binding energy or atomic species. That prediction, however, fails at very high $n$ once photoabsorption channels start opening. We observed a significant decrease in ionization fields 4 and 6 microwave photons below the ionization limits at 38 and $80 \mathrm{GHz}$ respectively. At this point, atoms excited an integral number of photons below the limit acquire a significant probability to make a sequence of transitions by absorbing microwave photons with subsequent photoionization. The photoionization rates at small fields that we observed at 80 $\mathrm{GHz}$ for atoms tuned one microwave photon below the limit are in an excellent agreement with the prediction of the perturbation theory calculated by the Fermi's Golden Rule.

In all of the described above experiments, a fraction, $\sim 10 \%$, of the atoms was found trapped in extremely high-lying states - a phenomenon never observed before for long microwave pulses and low initial Rydberg states. This observation is of a major interest and will be the main topic of the next two chapters. 


\section{Chapter 4}

\section{Spectrum of quasi-stable states in strong microwave fields}

\subsection{Introduction}

An intense laser pulse ejects the electron from the ion core either in a single field cycle, through tunnel ionization, or over many cycles, via multiphoton ionization. In either case, the electron departs from the core with a range of energies into the long range tail of the coulomb potential, where the electron is nearly free, and there is no cycle average energy exchange with the laser field. Above the ionization limit the range of energies appears explicitly in the form of above threshold ionization [6], but the energy range extends below the limit as well. An electron with an energy below the limit is reflected by the coulomb potential and returns to the core in one Kepler period [27, 29, 30]. If the laser pulse is over before the return, the electron obviously remains bound. If the laser pulse is not over, rescattering of the electron from the core can lead to superponderomotive ATI, recombination and high harmonic generation.

Microwave ionization of Rydberg atoms exhibits many similarities to multiphoton ionization of ground state atoms. In particular, recent microwave ionization experiments have shown that $10 \%$ of the atoms remain bound in high-lying states at fields orders of magnitude beyond those required for static field ionization [57, 23]. Furthermore, electrons in the highlying states remain bound even if they return to the ionic core during the microwave pulse, essentially the same result as observed in the laser experiments [58]. While quasi-stable 
high-lying states are formed in both the laser and microwave experiments, an attractive feature of the microwave case is that it is possible to probe them spectrally. Here we report the optical excitation spectra of $\mathrm{Li}$ and $\mathrm{Na}$ Rydberg atoms in a strong 38 and $80 \mathrm{-GHz}$ microwave field. Our spectra demonstrate that the surviving atoms result from excitation to microwave dressed states just below the limit. Specifically, the spectra consist of series of peaks separated by the microwave frequency. These observations imply that the atoms are in quasi-stable states in which the stability derives largely from the electron's spending most of its time far from the ionic core. Nonetheless, the atom survives the electron's revisiting the core.

\subsection{Spectrum of quasi-stable states of $\mathrm{Li}$ in $38-\mathrm{GHz}$ field}

Recent microwave experiments showed a structure of peaks spaced by a microwave photon in the vicinity of the ionization limit when the laser pulse is during the microwave

pulse $[23,38,59]$. In order to study that phenomenon thoroughly, we excited a wide range of Rydberg states of lithium atoms in the presence of a strong 38-GHz microwave field. Our experiment was conducted with a very small static field that was shown to affect the observed structure of peaks. The main goal of this experiment was to study how far below and above the ionization limit in the spectrum that structure can extend, what the final states distribution of atoms is, how atoms react to changes in the external environment such as static fields or longer microwave pulses. In the sections which follow, we describe our experimental approach, present our experimental observations, and draw conclusions.

\subsubsection{Experimental Approach}

A detailed discussion of the experimental apparatus is presented in Chapter 2. The setup used in this experiment is similar to the one described in section 3.3.1, with the only difference that the microwave pulse starts before the laser excitation and is on after it for about 30 ns, before it turns off with 15-ns fall time as shown in Fig. 4.1. In brief, a thermal beam of Li atoms in its ground state passes through the antinode at the center of a 38.34- 


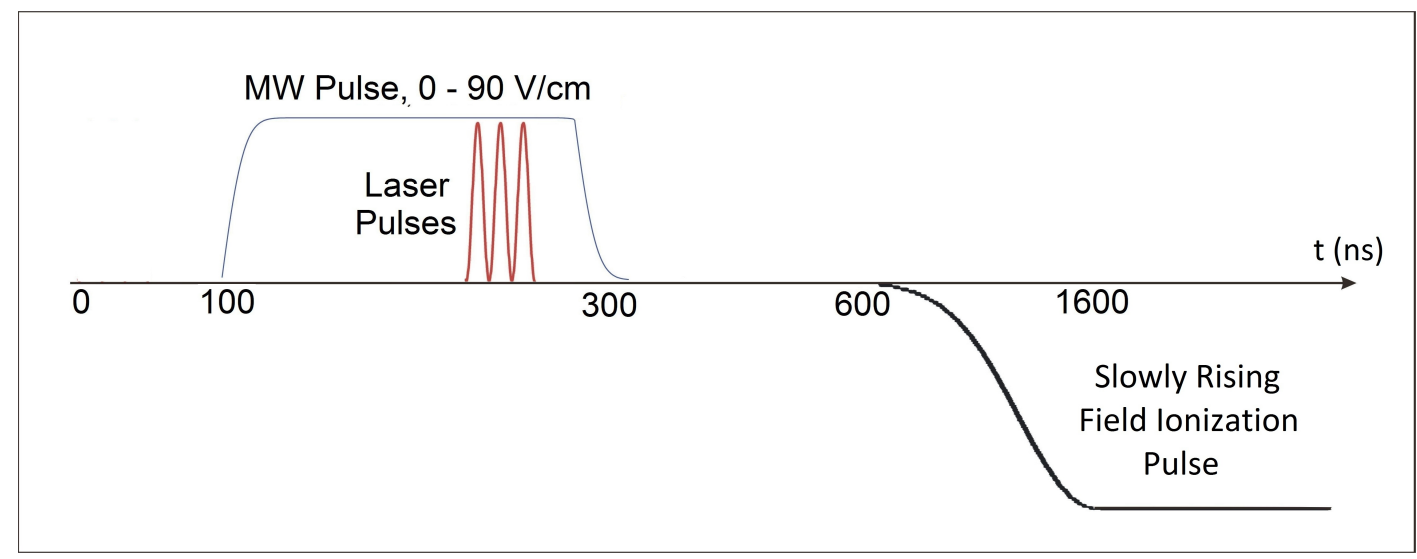

Figure 4.1: Experimental timing diagram for the investigation of microwave spectrum in Li by a $38.3-\mathrm{GHz}$ microwave field

$\mathrm{GHz}$ Fabry-Perot cavity where the atoms are excited via the $2 s \rightarrow 2 p \rightarrow 3 s \rightarrow n p$ transitions in the presence of the microwave field. The microwave system generates a $38.34-\mathrm{GHz}$ pulse with a variable width and 0 - to $90 \mathrm{~V} / \mathrm{cm}$ amplitude. A stray field of $9 \mathrm{mV} / \mathrm{cm}$ is present in the excitation volume, and well defined static fields can be added. After the end of the microwave pulse, typically $300 \mathrm{~ns}$ later, atoms are field ionized by the FIP, which reaches a maximum of $110 \mathrm{~V} / \mathrm{cm}$ with a $1 \mu$ s rise time. It is adequate to fully ionize states of $n>43$. The electrons ejected by the ionization field are detected by a dual micro-channel plate detector. Due to slow rising time of the FIP, signal from different states is time-resolved, so we can set up an integration gate in such a way as to record signal only from particular atomic states. For example, if a very narrow gate is utilized, only the high-lying states with $n>210$ will be detected.

\subsubsection{Experimental Observations}

In Fig. 4.2 we show the spectra obtained by scanning the frequency of the third laser in the presence of microwave fields from 0 to $45 \mathrm{~V} / \mathrm{cm}$. The gate of the integrator is wide enough to collect the field ionization signals of all states of $n>43$, that is of energies greater than $-1500 \mathrm{GHz}$. We specify energies relative to the zero field ionization limit. In zero microwave field we observe resolved bound Rydberg states at energies lower than $-300 \mathrm{GHz}$ and a flat signal at higher energies, where the spacing between adjacent levels is smaller 
than the $8-\mathrm{GHz}$ linewidth of our laser, which occurs at $n \approx 100$. At an energy of $-45 \mathrm{GHz}$, the signal starts falling to zero because photoelectrons are not detected when the laser is tuned over the ionization limit. Due to the finite linewidth of the laser, the signal drops to zero with a finite slope, and we denote the middle of that slope as the ionization limit. The limit is depressed due to the stray static field in the interaction region mentioned above. We estimate the stray field using $\Delta W=2 \sqrt{E}$. Typically, the data are taken when the depressed ionization limit is at $-18 \mathrm{GHz}$, which corresponds to a stray field of $9 \mathrm{mV} / \mathrm{cm}$. As shown by

\section{Number of MW photons relative to the Ionization Limit}

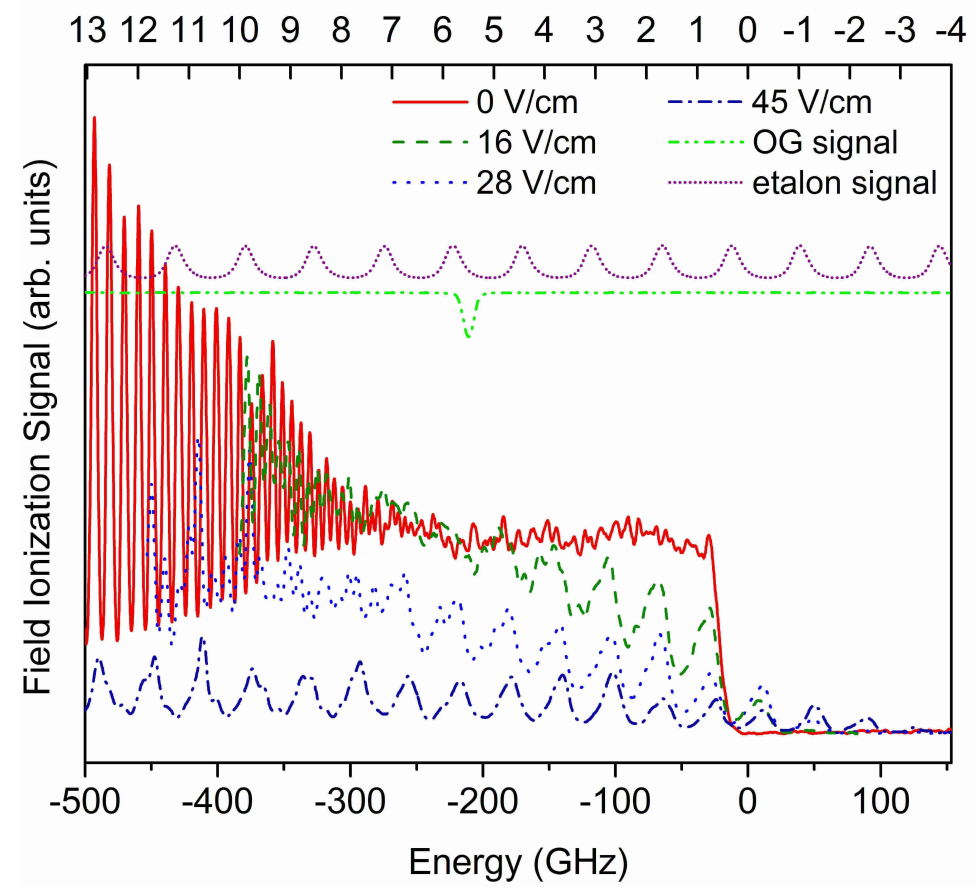

Figure 4.2: Field ionization signal as a function of laser frequency tuning, in terms of energy relative to the ionization limit (IL), after exposure to a microwave pulse of amplitude: $0 \mathrm{~V} / \mathrm{cm}, 16 \mathrm{~V} / \mathrm{cm}, 36 \mathrm{~V} / \mathrm{cm}$, and $45 \mathrm{~V} / \mathrm{cm}$. The horizontal axis is calibrated by the etalon and optogalvanic signals.

Fig. 4.2, the presence of the microwave field leads to an obvious modulation of the spectrum at the microwave frequency. There are several points to note regarding Fig. 4.2. First, as the microwave field is raised the regular structure persists further both above and below the ionization limit, as observed previously $[23,38,59]$. Second, in the $16-\mathrm{V} / \mathrm{cm}$ spectrum of Fig. 4.2 the high frequency side of the peak at the limit coincides with the drop in signal at the limit in the zero (microwave) field spectrum of Fig. 4.2. In addition, the peaks in the 
signal near the limit are asymmetric, but the asymmetry disappears at higher microwave fields. This asymmetry is expected if the ionization occurs through photon absorption in the perturbative limit (low power), since in this case the photoabsorption rate scales as $1 / n^{3}$. This results in a higher photoionization rate for an electron bound by $95 \%$ of the photon energy than for one bound by $5 \%$ of the photon energy. Finally, the entire set of peaks moves to higher energy due to the ponderomotive energy shift. For the highest microwave field we have used, $90 \mathrm{~V} / \mathrm{cm}$, we observe a ponderomotive shift of $15 \mathrm{GHz}$, confirming our field calibration.

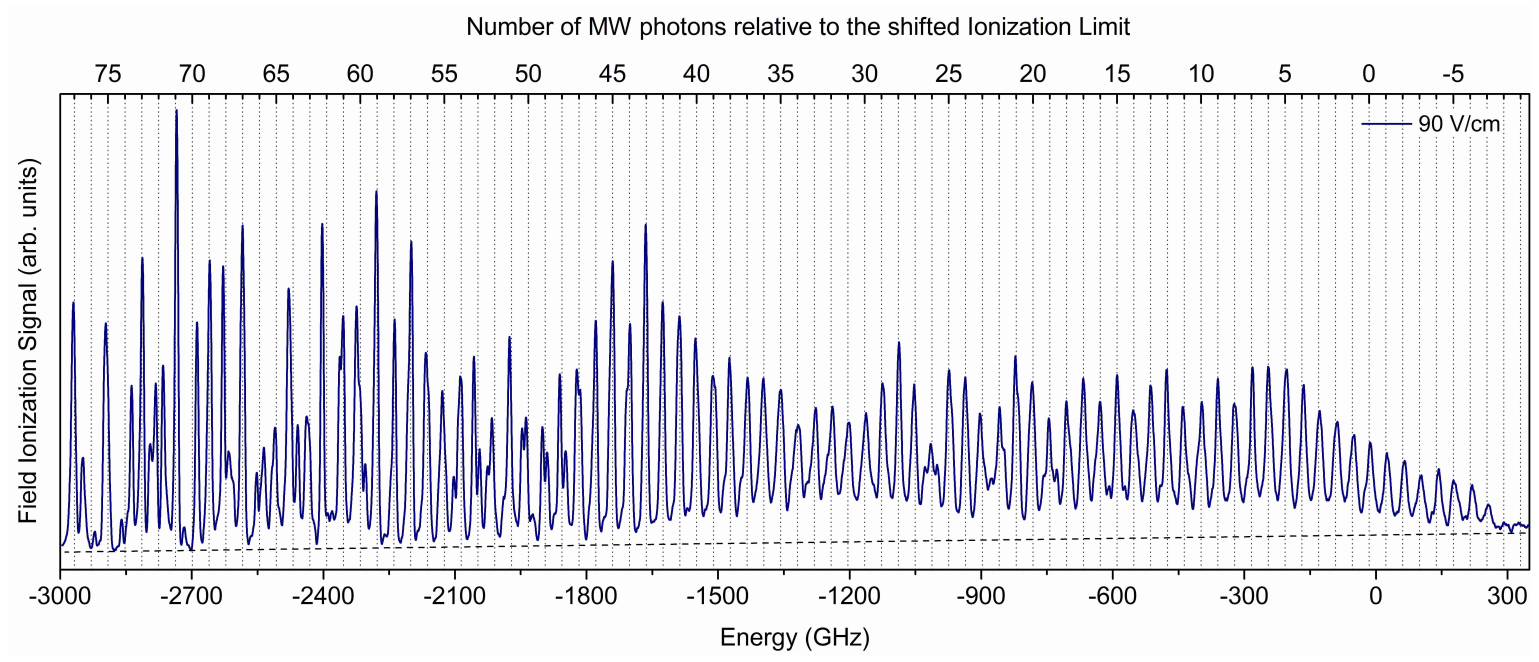

Figure 4.3: Field ionization signal as a function of laser frequency tuning. $90-\mathrm{V} / \mathrm{cm}$ microwave field is on during the laser excitation. A uniform structure of peaks appears above the IL and continues smoothly across the limit down to energies where $\Omega=1 / 5$. As shown by the vertical dotted lines spaced by 38.34 $\mathrm{GHz}$, the structure is at the microwave frequency.

The higher field spectra of Fig. 4.2 show that the microwave structure appears not only near the ionization limit, but persists further below it as the field amplitude is increased. Fig. 4.3 shows a spectrum obtained when the atoms were exposed to a $90-\mathrm{V} / \mathrm{cm}$ pulse, the maximum available field amplitude. It is evident that the observed spectrum consists of a regular structure of peaks that are spaced by a microwave photon from each other as shown by dotted vertical lines. This structure starts about $300 \mathrm{GHz}$ above the ionization limit and, surprisingly, extends not only to $\Omega=1$ at $-1050 \mathrm{GHz}$, but much further down to -3000 where $\Omega=0.2$. A comparison of this spectrum and a spectrum of zero-field Rydberg states is presented in Fig. 4.4. Indeed, we see that the microwave structure obtained with 


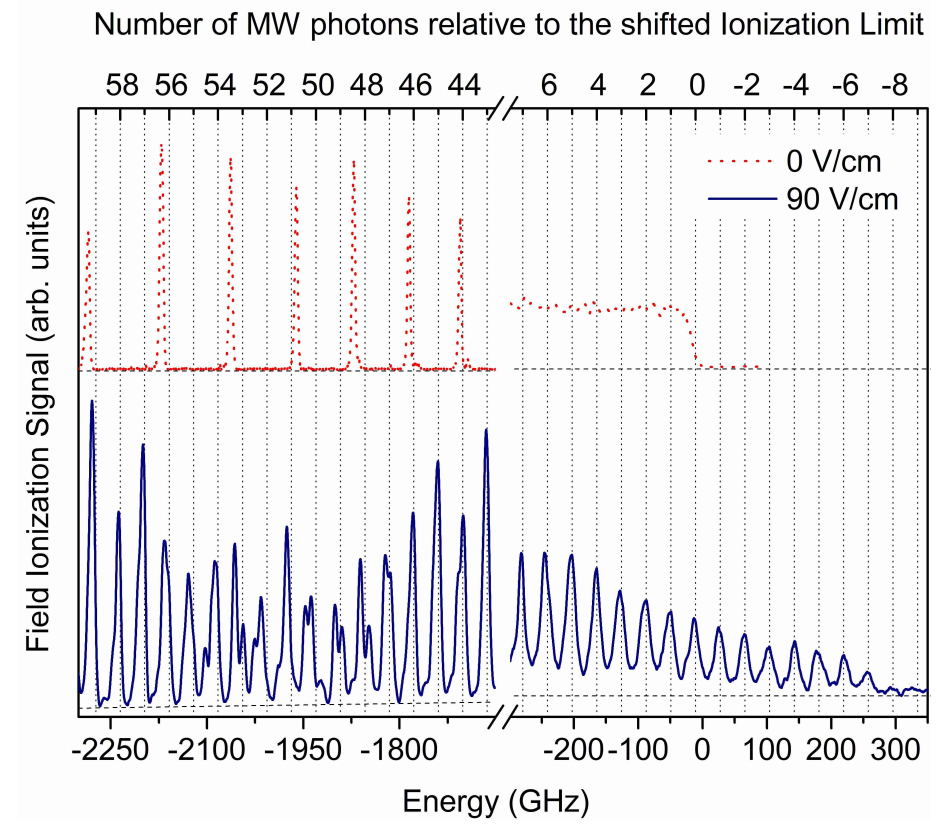

Figure 4.4: Field ionization signal as a function of laser frequency tuning. Top trace: excitation in zero-field (scaled by a factor of 5 ); bottom scan: excitation in the presence of the $90-\mathrm{V} / \mathrm{cm}$ field. A break is shown on the horizontal axis for convenience.

the $90-\mathrm{V} / \mathrm{cm}$ microwave field persists even at energies where the spacing between Rydberg states is larger than $38 \mathrm{GHz}$, and no trace of Rydberg states is detected. Moreover, we observe non-zero signal at frequencies that are not resonant with Rydberg states. That can be explained by the fact that a periodic field dresses zero-field states that develop extra levels, or sidebands, spaced an integral number of microwave photons. When the microwave field is on during the laser pulse, excitation to these sideband energy levels can be populated by atoms. It is yet unclear, though, which atomic states are dressed by the microwave field to form the spectrum that we observe in Fig. 4.3.

Inspection of the time resolved field ionization signal obtained in the $90-\mathrm{V} / \mathrm{cm}$ trace of Fig. 4.3 shows that all the signal comes very early in the FIP and belongs to atoms in very highly excited states. Accordingly, we narrowed the gate of the integrator to $50 \mathrm{~ns}$ to detect only atoms bound by less than $75 \mathrm{GHz}$ and recorded the spectra shown in Fig. 4.5. When there is no microwave field, we only detect a signal when the laser is tuned within $75 \mathrm{GHz}$ of the depressed ionization limit at $-18 \mathrm{GHz}$. It is important to bear in mind that $75 \mathrm{GHz}$ is an upper limit to the binding energy of the atoms we detect. As the microwave 


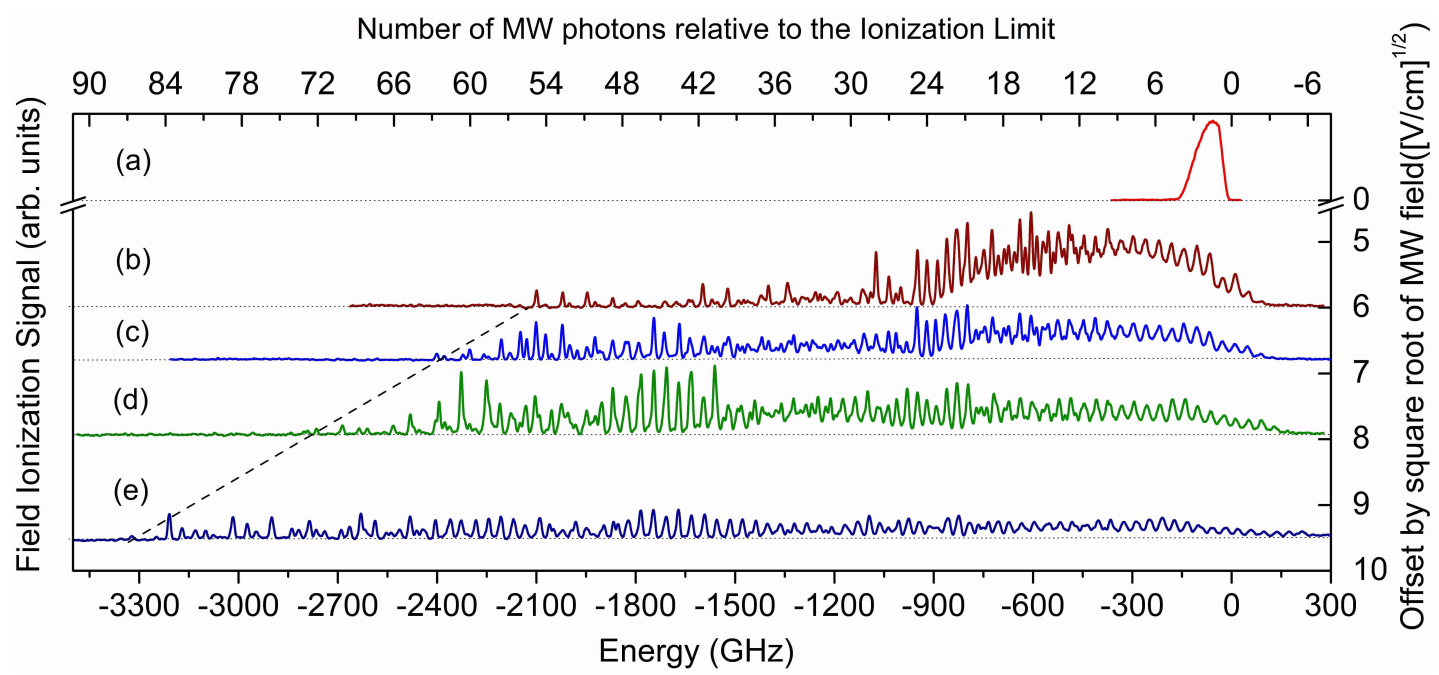

Figure 4.5: Field ionization signal as a function of laser frequency, after exposure to a microwave pulse of amplitudes: (a) $0 \mathrm{~V} / \mathrm{cm}$, (b) $36 \mathrm{~V} / \mathrm{cm}$, (c) $45 \mathrm{~V} / \mathrm{cm}$, (d) $63 \mathrm{~V} / \mathrm{cm}$, and (e) $90 \mathrm{~V} / \mathrm{cm}$. A 50 -ns integration gate is used to detect only atoms bound within $75 \mathrm{GHz}$ of the IL. The zero field spectrum is scaled by a factor of $1 / 3$ to fit the graph. Horizontal dotted lines show the zeroes of the corresponding spectra. The traces are offset by the square root of the microwave field. The broken line connects the onsets of the peaks in the different spectra, and the energy of the onset obeys the relation $W=-4 \sqrt{E}$.

field amplitude is increased the spectrum extends progressively further above the limit, and more interestingly, to very deeply bound states. The broken line of Fig. 4.5 connects the onsets of the peaks in the spectra, and the energy of the onset is linearly dependent on the square root of the microwave field. Converting from the laboratory units given in Fig. 4.5 to atomic units shows that the energy of the onset is given by $W=-4 \sqrt{ } E$, almost exactly twice the binding energy for classical field ionization. It is not clear to us why the onset of the spectra occurs at $W=-4 \sqrt{ } E$, but measurements at a lower frequency might clarify this point, as at $80 \mathrm{GHz}$, there is no clearly defined onset.

The regularity of the structure at the microwave frequency extends almost as far as the spectra shown in Fig. 4.5. In Fig. 4.6 (b) we again show an expanded view of the $90-\mathrm{V} / \mathrm{cm}$ spectrum of Fig. 4.5, as well as a set of vertical dotted lines spaced by the microwave frequency to show the regularity of the microwave induced structure down to an energy of $-2400 \mathrm{GHz}$. In Fig. 4.6 (a) we compare it to a spectrum recorded in the presence of a $90-\mathrm{V} / \mathrm{cm}$ microwave field when the atoms are ionized immediately, $\sim 10 \mathrm{~ns}$, after the microwave pulse by a field pulse which ionizes atoms bound by up to $1500 \mathrm{GHz}$. Electrons produced by microwave ionization do not leave the interaction region in this time, so the 


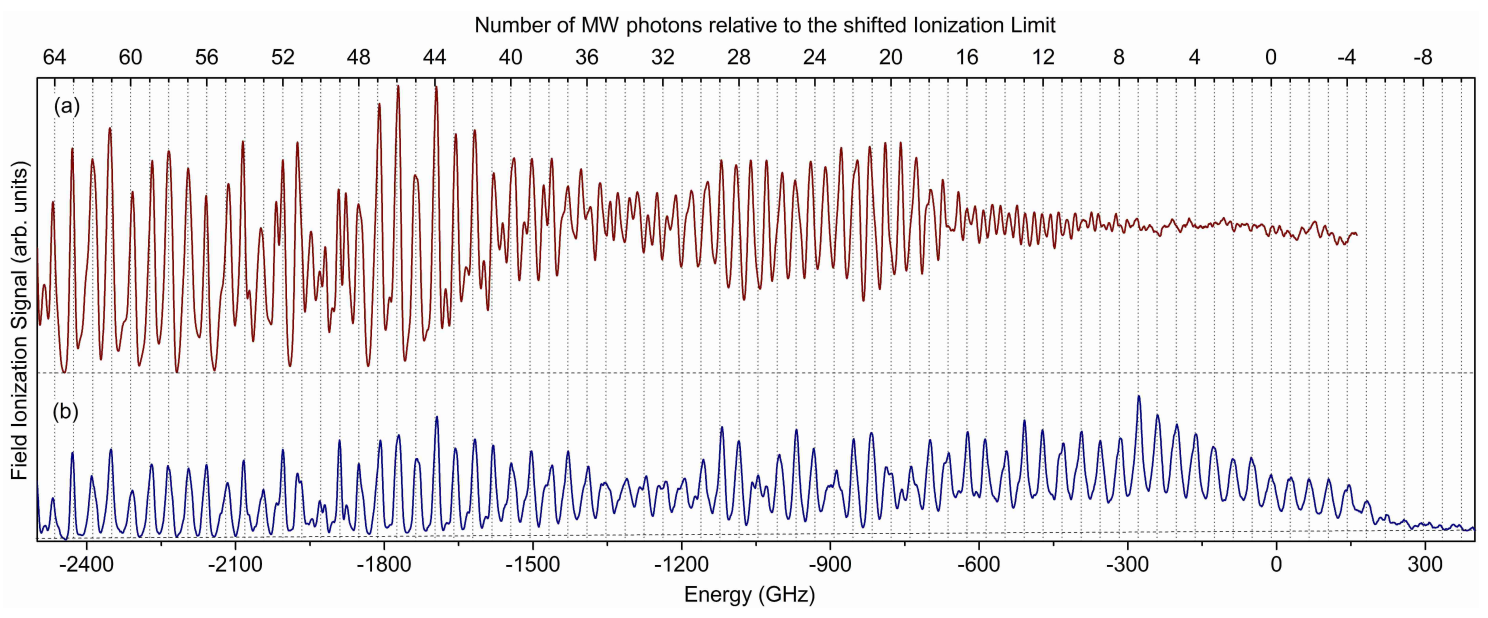

Figure 4.6: Field ionization signals as a function of laser frequency for excitation in the presence of a microwave pulse of amplitude of $90 \mathrm{~V} / \mathrm{cm}$. (a) The total photoabsorption spectrum when detecting all energies above $n=43$. (b) Spectrum obtained when detecting only the high-lying states. The total photoabsorption spectrum is scaled by a factor of eight to fit the graph. Horizontal dashed lines show the zeroes of the corresponding spectra. The total photoabsorption spectrum also presents the $38 \mathrm{GHz}$ structure at energies below $-1500 \mathrm{GHz}$ since only atoms transferred to higher lying states are detected.

resulting spectrum is identical to one obtained by detecting ions. At energies below -1500 $\mathrm{GHz}$ not all the initially excited atoms are detected, only ones which have been transferred to within $1500 \mathrm{GHz}$ of the ionization limit. Thus the spectrum has the same modulation as the spectrum of Fig. 4.6 (b), in which the field pulse is $300 \mathrm{~ns}$ after the microwave pulse, as shown in Fig. 4.1. At energies above $-1500 \mathrm{GHz}$ there is, in general, no obvious connection between the spectra of Figs. 4.6 (a) and 4.6 (b). The microwave frequency matches the Kepler, $\Delta n=1$, frequency at $-1050 \mathrm{GHz}$, which is probably the origin of the structure in Fig. 4.6 (a). In any event, the deep modulation of the total number of atoms excited, shown in Fig. 4.6 (a), introduces a beat note into the spectrum of Fig. 4.6 (b).

Several observations suggest that the spectra are tied to the ionization limit. First, the spectra of Fig. 4.5 were recorded by detecting very high-lying states. Second, in Figs. 4.2 and 4.5 the extent of the spectra above and below the limit increases with the microwave field amplitude. Finally, the $16-\mathrm{V} / \mathrm{cm}$ spectrum of Fig. 4.2 appears to have a peak at the ionization limit. As an explicit test of this notion, we have recorded spectra in the presence of small static fields which depress the limit by different amounts. In Fig. 4.7 we show segments of spectra recorded in static fields of up to $55 \mathrm{mV} / \mathrm{cm}$. In particular, we 


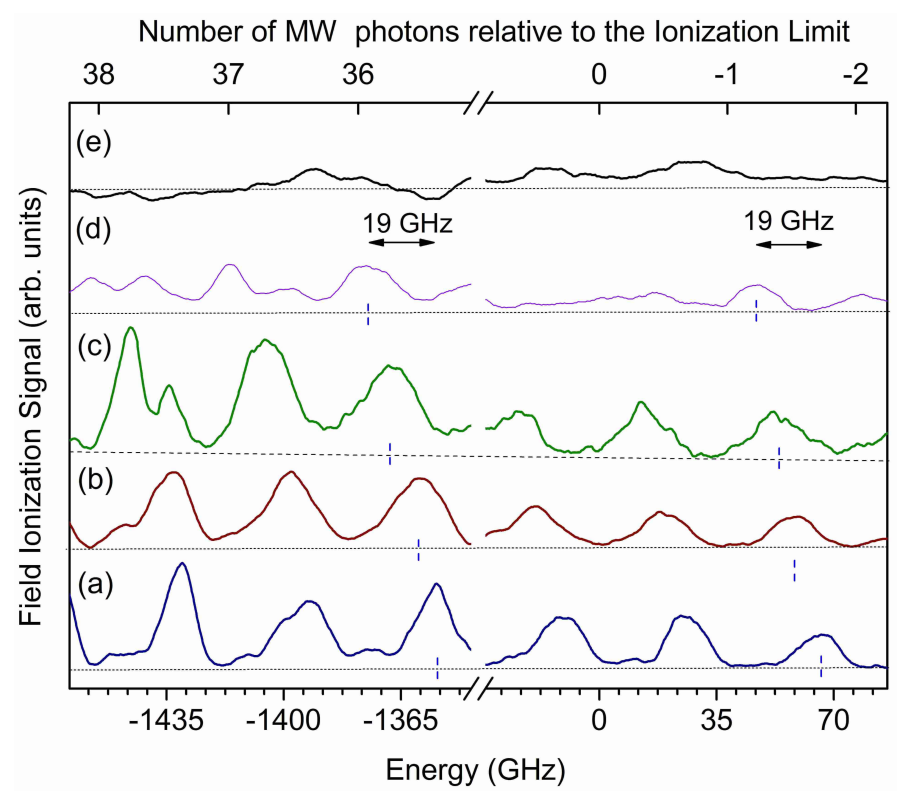

Figure 4.7: Field ionization signal as a function of laser frequency tuning after exposure to a microwave pulse of $90 \mathrm{~V} / \mathrm{cm}$ amplitude for different static fields in the interaction region: (a) $9 \mathrm{mV} / \mathrm{cm}$, (b) $17 \mathrm{mV} / \mathrm{cm}$, (c) $25 \mathrm{mV} / \mathrm{cm}$, (d) $40 \mathrm{mV} / \mathrm{cm}$, and (e) $55 \mathrm{mV} / \mathrm{cm}$. A break is shown on the horizontal axis for convenience. Horizontal dashed lines show the baselines of the corresponding spectra. Vertical blue lines intersect the horizontal baselines at points to which the ionization limit is depressed due to $W=2 \sqrt{ } E$ for the corresponding static field $E$. The double headed arrow $19 \mathrm{GHz}$ long is the difference in the depression of the limit by 40 and $9 \mathrm{mV} / \mathrm{cm}$ fields. It is apparent that peaks in the microwave structure also shift by $W$, irrespective of the laser frequency. Increasing the static field destroys the microwave structure, and the $55-\mathrm{mV} / \mathrm{cm}$ spectrum shows only noise, with no bound population detected.

show segments of the spectra at the limit and at the energy $-1400 \mathrm{GHz}$. At the limit the spectra shift to lower energy as the depressed limit shifts, and precisely the same shift is observed at $-1400 \mathrm{GHz}$. In short, the entire spectrum is tied to the ionization limit. The other important aspect of Fig. 4.7 is that the size of the peaks decreases with increasing static field, and the peaks essentially disappear at $55 \mathrm{mV} / \mathrm{cm}$. The dependence on the static field suggests that atoms which survive the microwave field are in states within one microwave photon of the limit. A static field of $50 \mathrm{mV} / \mathrm{cm}$ depresses the ionization limit by $41 \mathrm{GHz}$, almost exactly the microwave frequency. We chose the energy $-1400 \mathrm{GHz}$ for Fig. 4.7 because it was the lowest energy at which structure persisted up to a static field of $40 \mathrm{mV} / \mathrm{cm}$. Unfortunately, at this binding energy the Kepler frequency is close to the microwave frequency, and the observed structure is not simply composed of peaks displaced from the limit by multiples of the microwave frequency; there are small subsidiary peaks. The same phenomenon can be seen in Fig. 4.6 (b). 


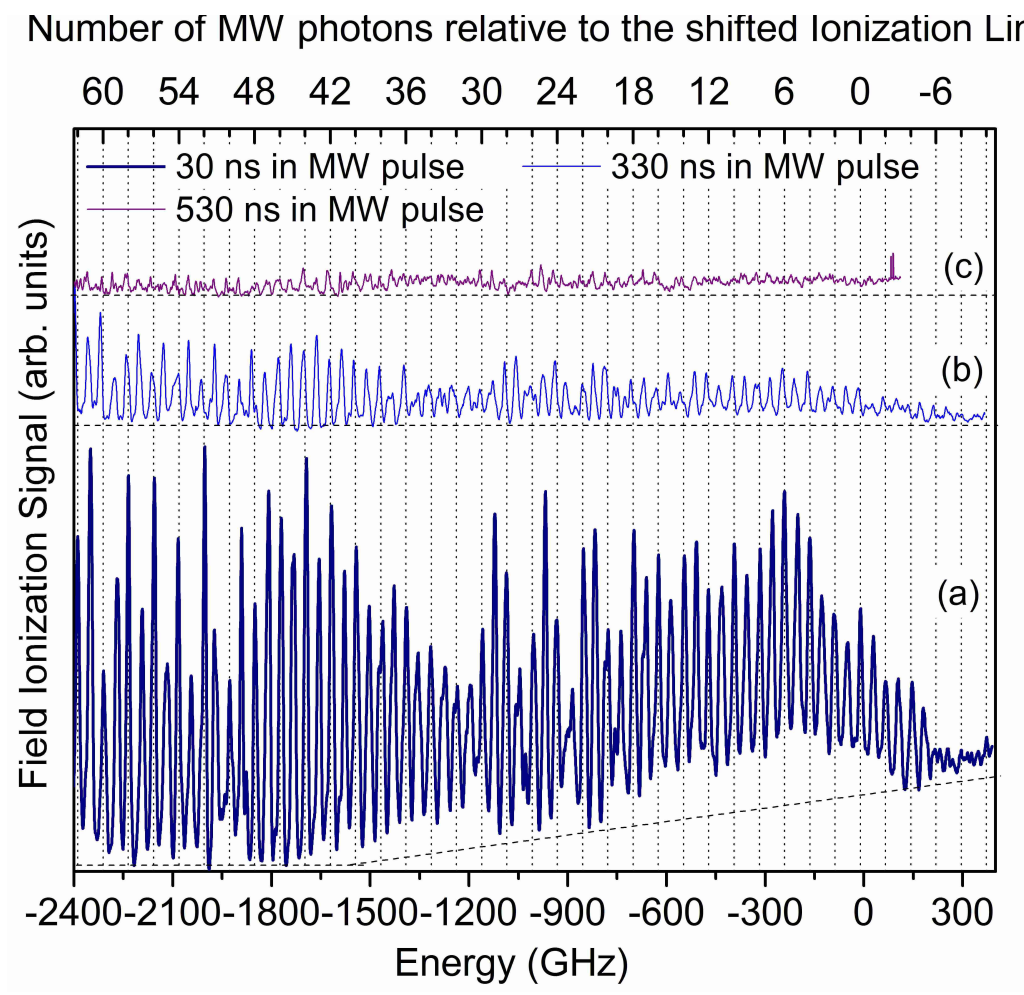

Figure 4.8: Field ionization signal as a function of laser frequency tuning. $90-\mathrm{V} / \mathrm{cm}$ microwave field is present during the laser excitation and is on for $30 \mathrm{~ns}$ (a), $330 \mathrm{~ns}$ (b), and $530 \mathrm{~ns}$ (c) after the last laser pulse. Only the extremely high-lying states are collected with a narrow integration gate. Horizontal dashed lines show zero of the corresponding spectrum. As shown by the vertical dotted lines spaced by two MW photons, the microwave structure is present in all traces, even when atoms spend $500 \mathrm{~ns}$ in the microwave field after the laser pulse ends.

The results of the observations discussed above suggest that atoms are trapped in the extremely high-lying states and survive the microwave field that has an amplitude several orders of magnitude larger than the ionization potential of these atoms. Consequently, it is interesting to increase the time that atoms spend in the microwave pulse to study how quickly atoms ionize from the high-lying states. We show several characteristic spectra are in Fig. 4.8. Surprisingly, atoms in the high-lying states bound only within two microwave photons of the ionization limit are very stable in the microwave field. We observe non-zero signal even when the width of the pulse exceeds $500 \mathrm{~ns}$. To determine the lifetimes of the atoms in the microwave field, we increased the delay time between the third laser pulse and turning off the microwave field and recorded the number of bound atoms as a function of the delay for several laser wavelengths. Typical spectral peaks, at $+60 \mathrm{GHz}$ and -2309 

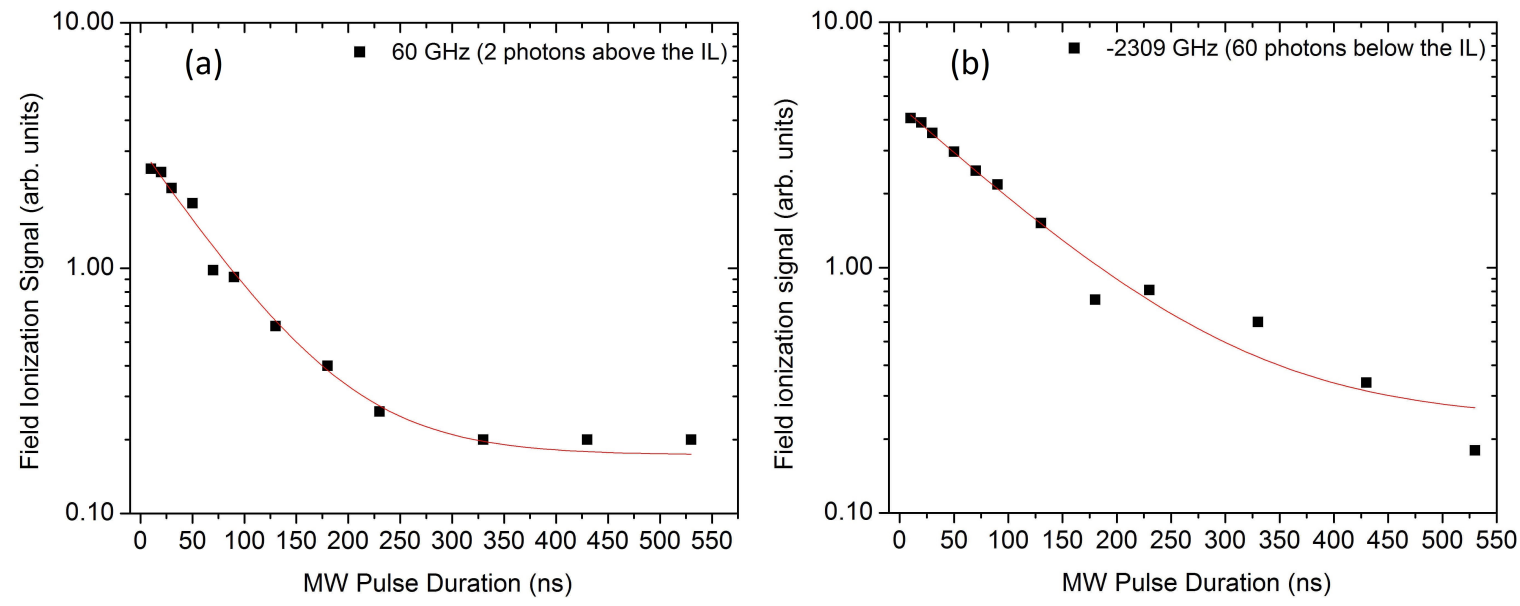

Figure 4.9: Field ionization signal as a function of microwave pulse duration after the laser excitation. The microwave pulse amplitude is $90 \mathrm{~V} / \mathrm{cm}$, and the laser frequency is fixed and tuned to (a) two mw photons above the ionization limit and (b) 60 photons below it. Only the very high-lying states are collected with a 50-ns gate. The lifetimes exhibit an initial rapid decay followed by a very slowly decaying tail. The solid lines represent fits to the form $A e^{-x / t}+c$. According to the fit, the lifetimes of decaying exponentials are 68(10) and 107(10) ns, respectively.

$\mathrm{GHz}$, exhibit two component decays, as shown in Fig. 4.9. In the former case the initial fast decay time is $68(10) \mathrm{ns}$, and in the latter it is $107(10) \mathrm{ns}$. In all cases the slow decay time is longer than $1 \mu \mathrm{s}$, so we fit the experimental data to a rapidly decaying exponential plus a constant. When the laser is tuned midway between the peaks similar decay curves, but with much smaller amplitudes, are observed. It is evident from Fig. 4.10 that, with the exception of the long decay time shown in Fig. 4.9, the decay times fall between 60 and 90 ns for all laser tunings.

Here we discussed the excitation of metastable Li Rydberg atoms in the presence of a strong $38.3 \mathrm{GHz}$ microwave field from low $\mathrm{n}$, where $\Omega=1 / 5$, to $300 \mathrm{GHz}$ over the ionization limit. We observe the uniform structure of peaks, spaced in energy by frequency of a microwave photon, and stretching for more than a $3500 \mathrm{GHz}$ range, when the 90$\mathrm{V} / \mathrm{cm}$ microwave pulse is present during the laser excitation, and the interaction volume has a small non-zero static field of about $9 \mathrm{mV} / \mathrm{cm}$. Such structure is observed in the ionization field due to an entrapment of atoms in the extremely high-lying states of $n>$ 215. The excitation is always to the ionization limit, modulo the microwave frequency, that is, to weakly bound Floquet states. Thus, an attractive alternative approach to this 


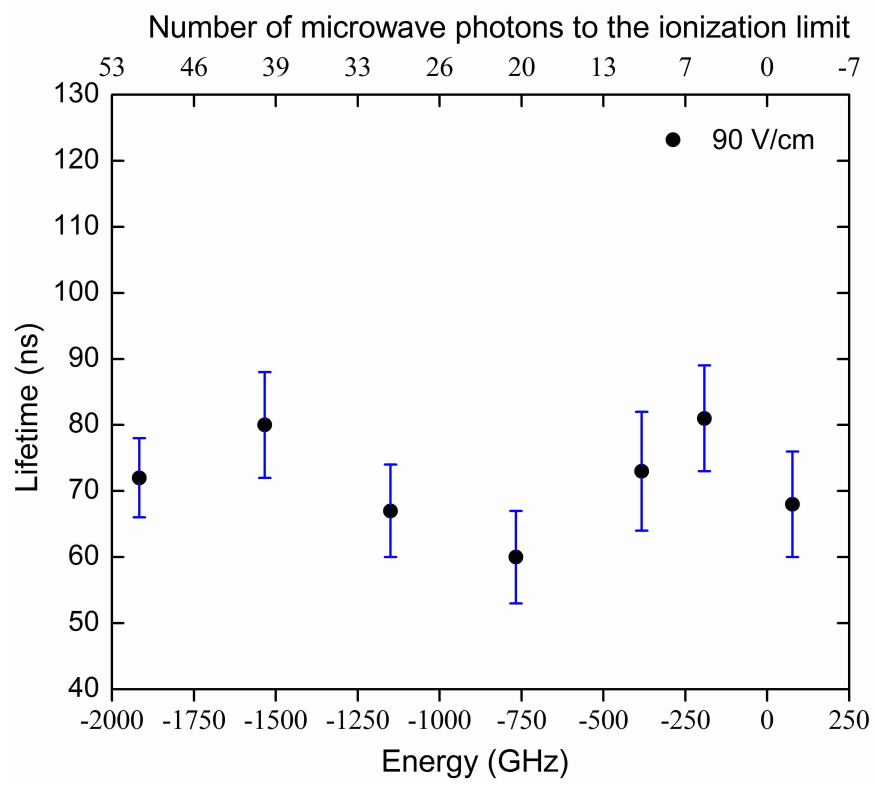

Figure 4.10: Extracted lifetimes of atoms in the high-lying states excited in the presence of the $90-\mathrm{V} / \mathrm{cm}$ field versus the laser tuning. The lifetimes of the fast decay, on average, fall between 60 and 90 ns for all laser tunings.

problem is the multichannel Floquet approach of Giusti-Suzor and Zoller [60]. The Floquet eigenstates contain the character of bound states very close to the limit, deeply bound states multiples of the microwave frequency below the limit, and continua multiples of the microwave frequency above the limit. Due to its having a continuum component, an initially bound eigenstate autoionizes into the continuum, just as in the quantum defect theory description of autoionizing states [61]. The survival of the resulting high-lying states is largely due to the fact that electron spends most of the time far from the core oscillating in the field. However, in the present case, the electron clearly have time to return to the core during the microwave pulse since the Kepler orbital period is short compared to the microwave pulse length, so the stability of atoms in the high-lying states in strong microwave fields is very surprising.

\subsection{A spectrum of the high-lying states of $\mathrm{Na}$ in $80-\mathrm{GHz}$ field}

An experiment conducted at $38 \mathrm{GHz}$ yielded a spectrum consisting of peaks spaced by a multiple of a microwave photon from the ionization limit when the microwave field is 
present during the excitation. Moreover, it turned out that the observed spectrum is due to the high-lying states trapped after the microwave pulse and very stable in it. The extent of the structure in the spectrum above the ionization limit was observed before and was wellexplained by a classical point of view by an implementation of the modified Simpleman's model $[38,23]$. However, it was unexpected to see that structure extending way below the limit where the spacing between zero-field Rydberg states is larger than the microwave frequency, and the classical description should not be applicable any more.

As our previous experimental efforts showed effects concerning the high-lying states to be more pronounced at higher microwave frequencies, we decided to study the spectrum of the high-lying states in 80-GHz fields. Besides, a new microwave system allowed to obtain a relatively high amplitude field that enabled an observation of the spectrum of the high-lying states as far as $-5000 \mathrm{GHz}$ below the limit $(n=26)$. This investigation mainly concerned a mechanism of how atoms reach the high-lying states when the laser is tuned in the $\Omega<1$ region, a response of the spectrum to a presence of static fields, lifetimes of the high-lying states in the microwave field and its dependence on the laser frequency.

\subsubsection{Experimental Approach}

A thermal beam of ground state sodium atoms crosses the microwave electric field antinode at the center of a 79.05-GHz Fabry-Perot microwave cavity. There atoms are excited to a Rydberg state by the sequence of transitions $3 s \rightarrow 3 p \rightarrow 3 d \rightarrow n f$ induced by two 20-ns dye laser pulses and a continuous-wave diode laser in the presence of the microwave field as shown in the timing diagram of Fig. 3.12 (a). About 300 ns after the end of the pulse, atoms are exposed to a $1-\mu$ s rise time field ionization pulse. Depending on the polarity of the FIP, either electrons or ions are sent upwards to a dual microchannel plate detector. The MCP signal is amplified, captured by a gated integrator or oscilloscope, and recorded in a computer for later analysis.

The Fabry-Perot microwave cavity consists of two brass mirrors $69.7 \mathrm{~mm}$ in diameter with $52-\mathrm{mm}$ radii of curvature. The on-axis spacing between mirrors is $90.51 \mathrm{~mm}$. The cavity is operated on the $\mathrm{TEM}_{048}$ mode at a frequency of $79.05 \mathrm{GHz}$ with $Q=8600$. 
The microwave system generates a $79.05-\mathrm{GHz}$ pulse with 0 - to $180 \mathrm{~V} / \mathrm{cm}$ amplitude and a variable width. The experiment is triggered at the $1-\mathrm{kHz}$ repetition rate of the frequencydoubled Nd:YLF laser. The excitation laser beams are sent to the vacuum chamber, through 1-cm holes in the side plates surrounding the cavity, and are focused to less than 1-mm diameter spots where they cross the atomic beam. The laser field and microwave field are polarized vertically.

\subsubsection{Experimental Observations}

Following the observation of a regular structure of peaks at $38 \mathrm{GHz}$ described in the previous section, we took a spectrum of the extremely high-lying states shown in Fig. 4.11. 79.05-GHz field was present during the excitation, and the amplitude of the FIP was chosen to ionize only states within $60 \mathrm{GHz}$ of the ionization limit. As always, when taking data from the high-lying states, we tried to null the static field, and $3-\mathrm{mV} / \mathrm{cm}$ field was present during the measurements. The $170-\mathrm{V} / \mathrm{cm}$ microwave field ponderomotively shifts the ionization limit by $12 \mathrm{GHz}$, and, in Fig. 4.11, we introduce dotted vertical lines spaced by $79 \mathrm{GHz}$ starting from the first peak below the ionization limit. The observed spectrum is similar to the one obtained at $38 \mathrm{GHz}$ : a regular structure of peaks is recorded, and each peak is spaced an integral number of microwave photons from the first peak below the limit as shown by vertical lines. We observe about six peaks above the ionization limit which is consistent with Shuman's et al. prediction based on the modified Simpleman's model [38]. The regular structure extends to about $-5000 \mathrm{GHz}$ below the limit $(\Omega=0.2)$ with a certain amplitude modulation: there is a pronounced waist at $-3400 \mathrm{GHz}$, and a large peak at $-2300 \mathrm{GHz}$ is due to a two photon excitation.

How atoms reach the high-lying states from an initial state is still unclear. One of the possibilities is a sequential excitation through transitions to $n+1$ and higher zero-field Rydberg states. It was shown before that an excitation spectrum of Na Rydberg states in a 15-GHz microwave field yields a regular structure of peaks spaced by a microwave photon when Stark manifolds of different zero-field Rydberg states, due to a static field of the same amplitude, overlap [19]. At this point, sidebands overlap as well, and a transition from $n$ to 


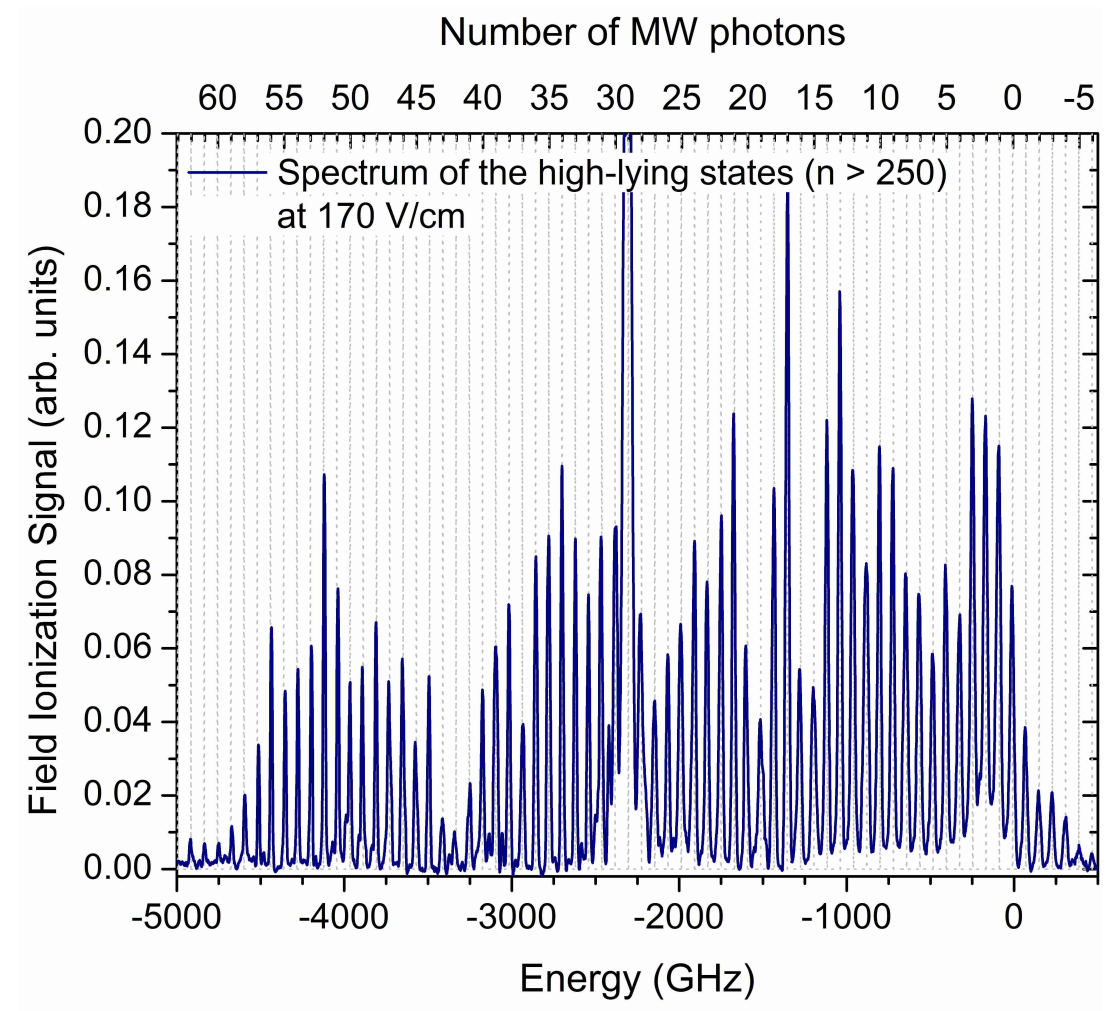

Figure 4.11: Field ionization signal as a function of the laser frequency, in terms of energy relative to the ionization limit. Electrons are detected, and the FIP amplitude is lowered to ionize only the high-lying states within $60 \mathrm{GHz}$ of the IL. The microwave pulse starts before the first laser and is on for $30 \mathrm{~ns}$ after the last excitation laser with 15-ns fall time. A structure of peaks is observed with each peak spaced by a microwave photon of $79 \mathrm{GHz}$. The vertical lines are $79 \mathrm{GHz}$ apart from the first peak below the IL which is depressed by $12 \mathrm{GHz}$ due to the static field of $3-\mathrm{mV} / \mathrm{cm}$ and is ponderomotively shifted to higher energy by $\sim 10 \mathrm{GHz}$. The classical Kepler frequency equals the microwave frequency at $-1700 \mathrm{GHz}(n=44)$. The large peak at $-2300 \mathrm{GHz}$ is due to a two photon excitation.

$n+1$ Rydberg state is possible. Consequently, we hypothesized that, when the microwave field amplitude is large enough for the sideband manifold of the initial state to overlap with the manifold of the next one, atoms can absorb microwave photons making transitions to higher states until they are trapped in the extremely high-lying states. However, the results of the ionization experiment conducted at $80-\mathrm{GHz}$ did not confirm that assumption as was discussed in Section 3.4.2. Figs. 3.14, 3.15, and 3.16 showed that at $80 \mathrm{GHz}$, even when manifolds of sidebands overlap, first, sideband peaks are not pulled together to form a regular structure of peaks that are spaced exactly a microwave photon from each other as was seen at $15 \mathrm{GHz}$ [19]: at any microwave field strength we tried, sideband peaks remained at the same frequencies, namely, spaced an integer number of photons from the 


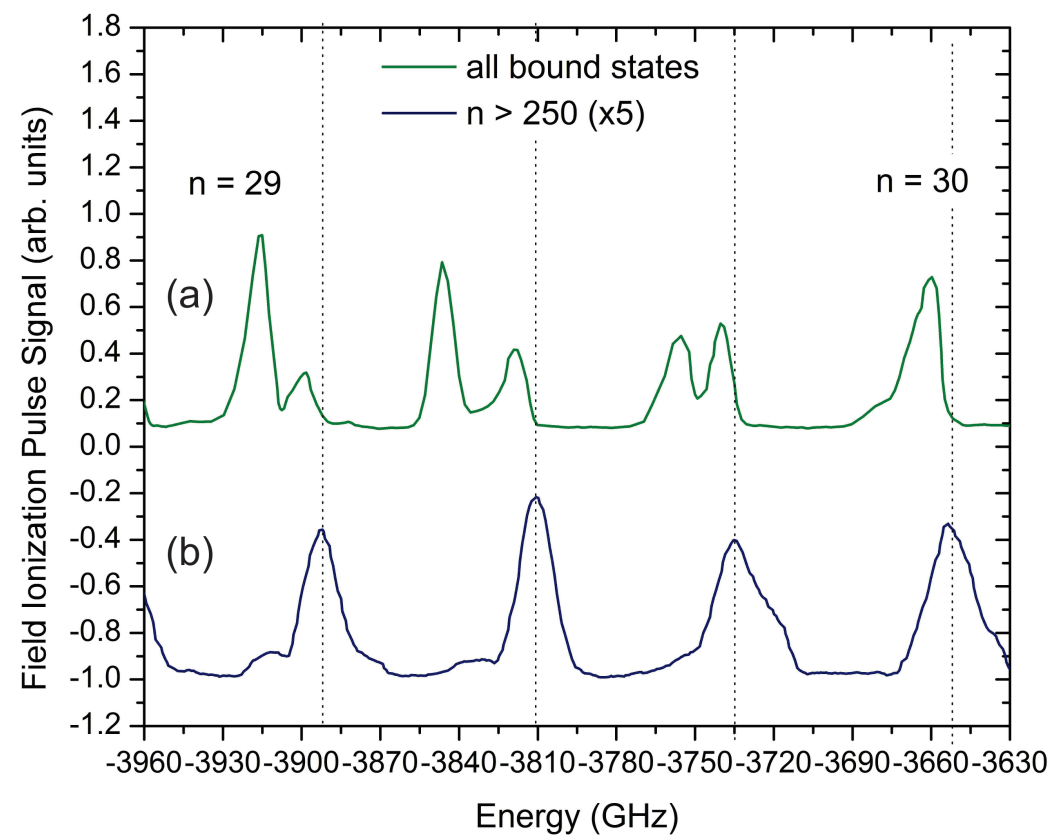

Figure 4.12: Field ionization signal as a function of the laser frequency between $n=29$ and 30 . Electrons are detected, and we used a large FIP with amplitude of $800 \mathrm{~V} / \mathrm{cm}$ to detect all bound states with $n>25$ (a) and a small FIP to detect only the high-lying states within $60 \mathrm{GHz}$ of the IL (b). The top spectrum yields two peaks at frequencies resonant with zero-field Rydberg states and additional peaks corresponding to sidebands spaced an integral number of microwave photons from them. It is clear that the number and position of peaks of spectra are different. Peaks in (a) are always spaced by a multiple of $79 \mathrm{GHz}$ from the corresponding Rydberg state, while the structure of peaks of spectrum (b) is always from the IL.

corresponding zero-field Rydberg state. Second, no population transfer from $n$ to $n+1$ states was observed. Moreover, a direct comparison of Figs. 3.14 and 4.11 shows that the total excitation spectrum and the spectrum of the high-lying states are different: peaks are not at the same frequencies, and both spectra yield a different number of peaks in the same energy range. To strengthen this point, in Fig. 4.12 we offer a collation of parts of spectra between $n=29$ and 30 taken by detecting electrons with a large $800-\mathrm{V} / \mathrm{cm}$ (a) and tiny $0.4-\mathrm{V} / \mathrm{cm}$ FIP (b). It is clear that peaks are at different frequencies: extra sideband peaks on the top spectrum are spaced from a corresponding Rydberg state, $n=29$ or 30 , while peaks of the bottom trace are tied to the ionization limit. Consequently, the production of the extremely high-lying states occurs not through sequential transitions from one zero-field Rydberg state to the next one, but, most likely, through a Floquet dressing of states close to the ionization limit which has to be investigated further.

Also, as discussed in section 3.4.2, the total excitation spectrum taken at $80 \mathrm{GHz}$ or the 


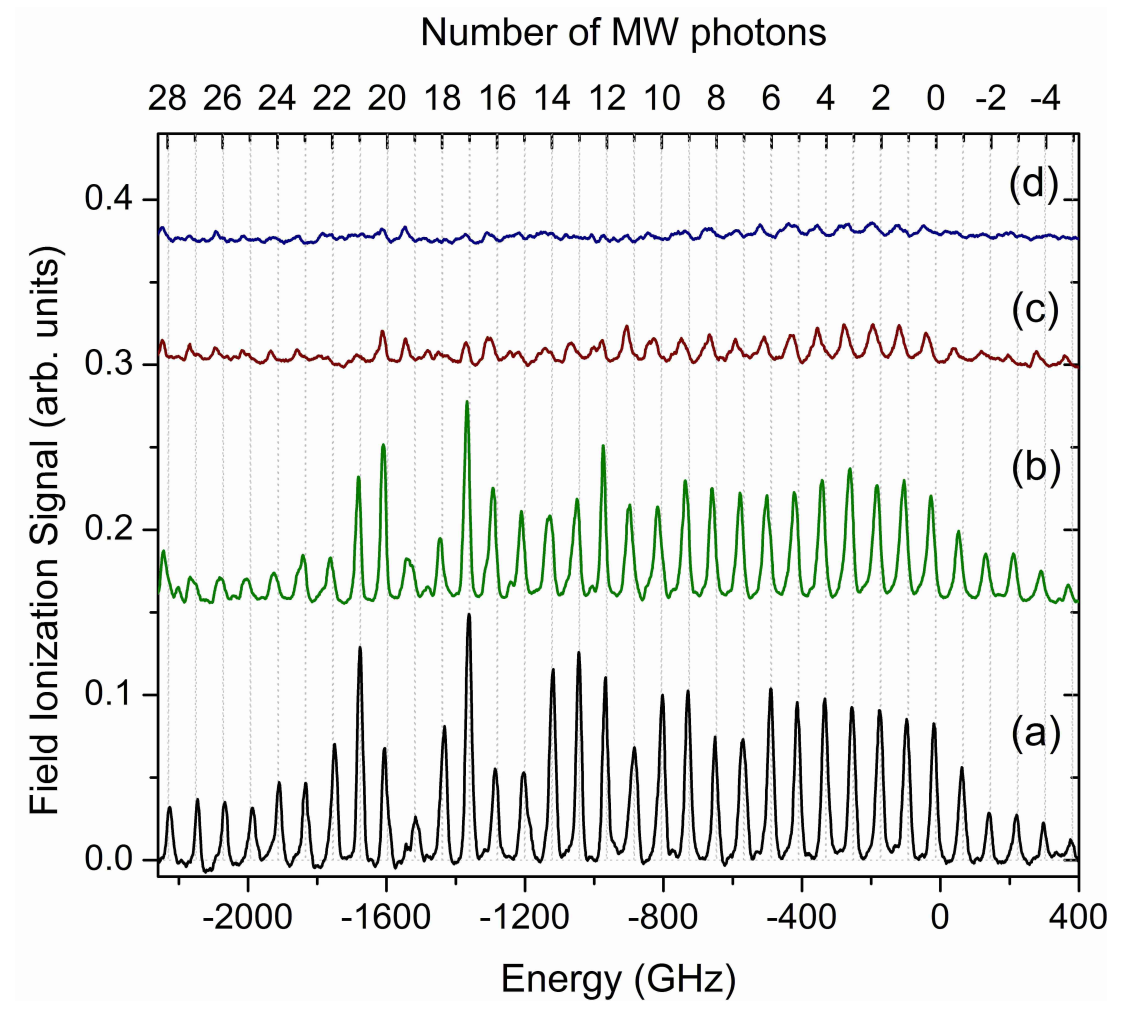

Figure 4.13: Field ionization signal as a function of the laser frequency in the presence of $4 \mathrm{mV} / \mathrm{cm}$ (a), $14 \mathrm{mV} / \mathrm{cm}$ (b), $36 \mathrm{mV} / \mathrm{cm}$ (c), and $57 \mathrm{mV} / \mathrm{cm}$ (d) static fields. Only electrons from the high-lying states within $60 \mathrm{GHz}$ of the IL are detected. The whole structure shifts by the same amount the IL is depressed. Less signal is observed at higher static fields, and at $75 \mathrm{mV} / \mathrm{cm}$ no signal was detected. The vertical lines are $79 \mathrm{GHz}$ apart from the first peak below the IL of the spectrum taken at the smallest static field. Spectra are vertically shifted to fit on the same graph.

spectrum of Fig. 4.12 (a) do not change when small static fields are added, while a spectrum of the high-lying states at $38 \mathrm{GHz}$ experienced a dc Stark shift along with the ionization limit. To investigate if the same is observed at $80 \mathrm{GHz}$, we recorded several spectra in the presence of static fields detecting only signal from the high-lying states. Fig. 4.13 shows such spectra for $4,14,36$, and $57-\mathrm{mV} / \mathrm{cm}$ static fields. At these fields, the ionization limit is depressed by $12,22,35$, and $44 \mathrm{GHz}$ respectively, and it is evident that peaks of spectrum (d) are shifted by $32 \mathrm{GHz}$ from peaks of (a) as well. Consequently, as in the 38-GHz experiment, the observed spectra of the high-lying states yield a structure of peaks that is tied to the ionization limit and is depressed along with it by static fields. A static field also decreases a number of atoms in the high-lying states, and no signal was detected when the field of $75 \mathrm{mV} / \mathrm{cm}$ was present, the field that ionizes population within $50 \mathrm{GHz}$ 
of the ionization limit. It is worth noting that peaks observed at binding energies far below the limit are affected by the static field more than peaks several microwave photons below it.

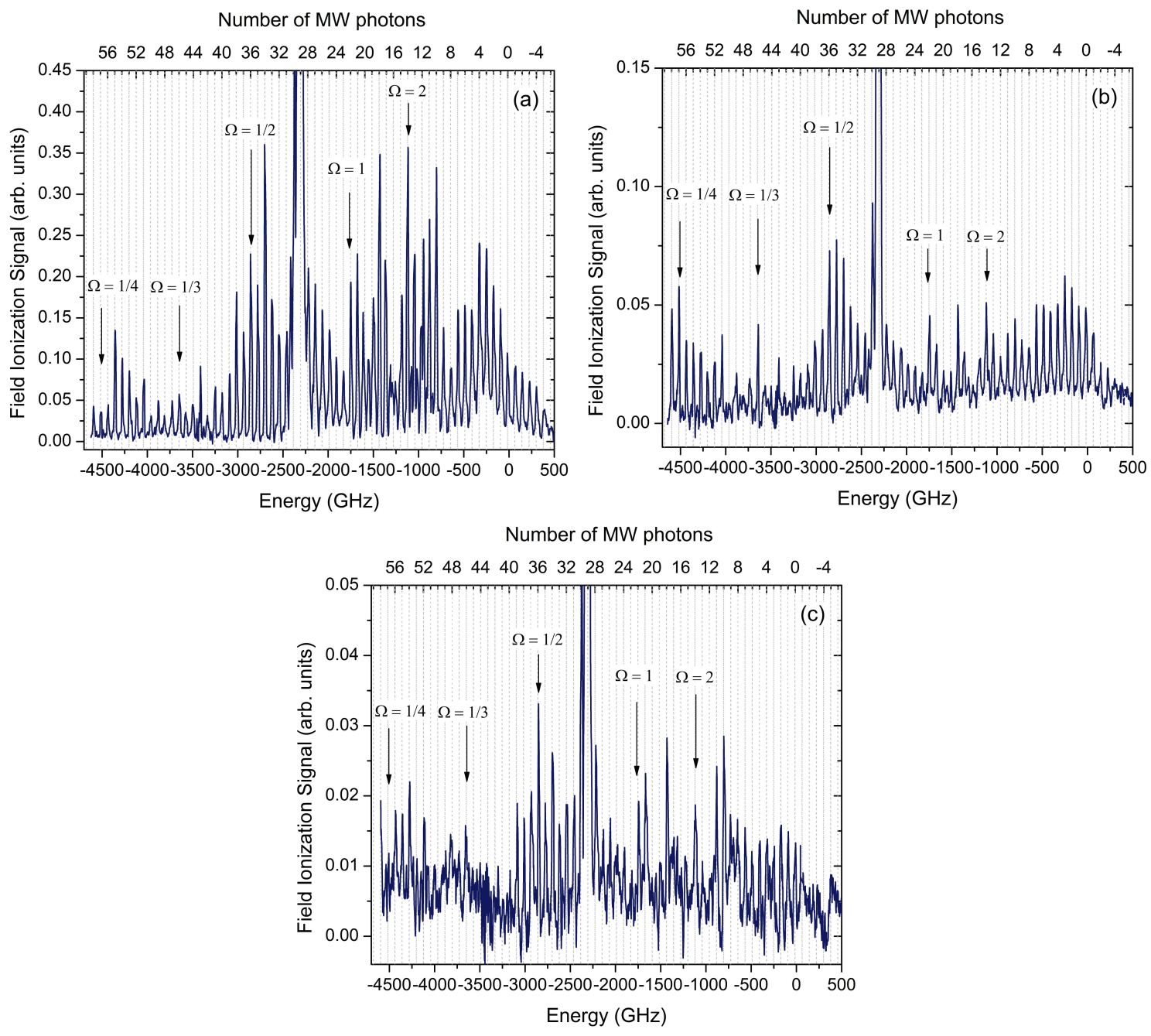

Figure 4.14: Field ionization signal as a function of the laser frequency in the presence of $3-\mathrm{mV} / \mathrm{cm}$ static field. Only electrons from the high-lying states within $60 \mathrm{GHz}$ of the IL are detected. The microwave pulse is on for $250 \mathrm{~ns}$ (a), $1000 \mathrm{~ns}$ (b), and $1700 \mathrm{~ns}$ (c) after the last excitation laser. Even after spending more than $1.5 \mu \mathrm{s}$ in the strong microwave field, a small number of atoms is detected in the high-lying states. For convenience, values of scaled frequency $\Omega$ are shown for some laser tunings.

Now that we established that atoms are found in the extremely high-lying states after the excitation in the presence of the microwave field that lasts about 30 ns after the excitation ends, it is interesting to investigate if atoms can survive in the microwave field longer. We recorded several spectra of the high-lying states for different widths of the microwave pulse 
conserving the delay between the end of the pulse and the FIP. Three characteristic cases are presented in Fig. 4.14 when atoms spend $250 \mathrm{~ns}$ (a), $1000 \mathrm{~ns}$ (b) and $1700 \mathrm{~ns}$ (c) in the $170-\mathrm{V} / \mathrm{cm}$ microwave field. It is evident that atoms in the high-lying states are very stable in the microwave field that substantially exceeds the ionization potential, and a small fraction of population can survive even very long pulses. However, it is also noticeable that atoms excited to the high-lying states at laser frequencies below $\Omega=1$ might have a larger lifetime. Thus, we directly measured lifetimes by fixing the laser at different peaks of the observed structure and recording the FIP signal as a function of the width of the microwave pulse. A resulting curve consists of two parts again: fast and very slow decay. For that reason we fit it by an exponent plus a constant as $A e^{-x / t}+B$. The resulting decay times $t$ are plotted in Fig. 4.15 as a function of laser tuning. In contrast to the result of the 38-GHz experiment, lifetimes of atoms in the microwave field increase for binding energies in the region $\Omega<1$.

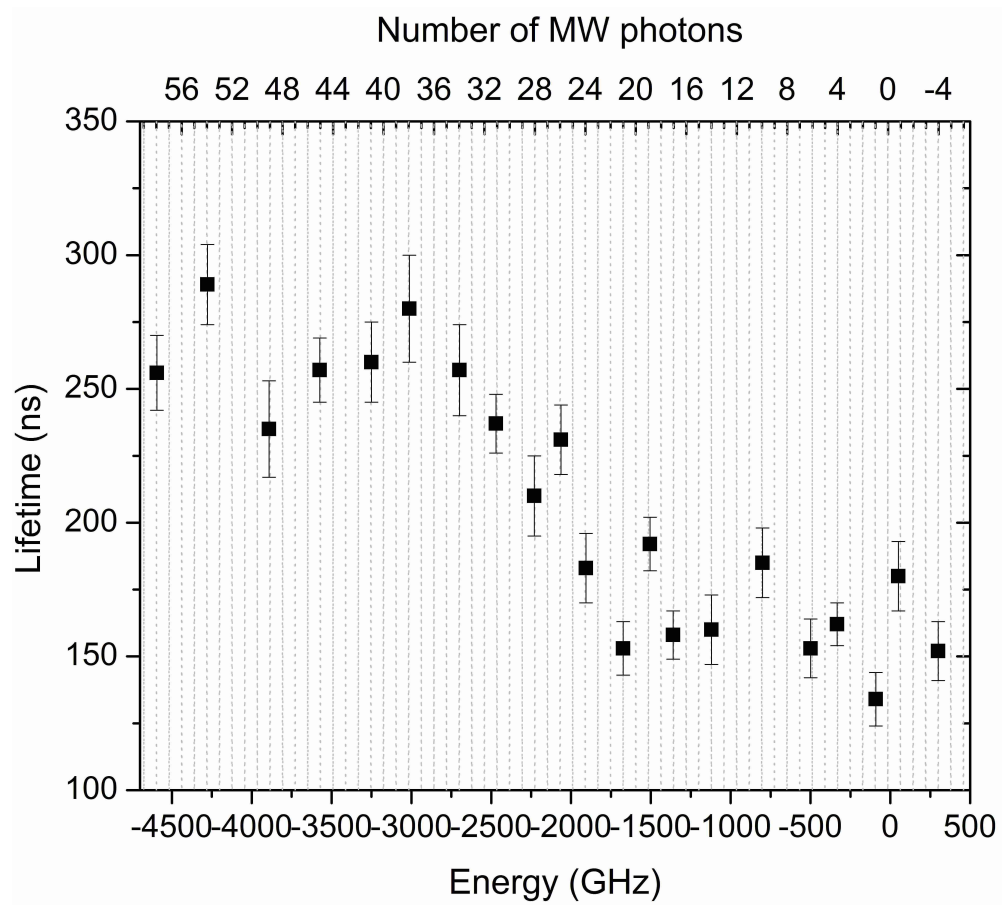

Figure 4.15: Extracted lifetimesof the extremely high-lying states as a function of the laser frequency for the $80-\mathrm{GHz}$ microwave field with $170-\mathrm{V} / \mathrm{cm}$ amplitude. At each binding energy, a decay curve was fitted with $A e^{-x / t}+B$ and a lifetime $t$, corresponding to the fast exponential decay, was measured. Lifetimes of peaks in the region $\Omega>1$ are, on average, the same and equal to 150 ns. Near $\Omega=1$ lifetimes increase before reaching a value of $260 \mathrm{~ns}$. 
By carefully inspecting Fig. 4.11, one can also notice that widths of the peaks change as the laser is tuned further below the limit. We used two ways to compare these widths: the Fast Fourier Analysis and a direct measurement of the width by deconvolution of the laser linewidth. First, we compared peaks near the ionization limit and far below it $(\Omega \approx 0.2)$ by performing a FFT of 5-6 peaks in these regions. The FFT analysis should yield one peak corresponding to the $80-\mathrm{GHz}$ spacing, if the structure is a pure sine wave, and more peaks, if the periodic structure is a sum of several sine waves and its peaks are narrower. In our case, the FFT demonstrated one large peak at the main frequency corresponding to the $80-\mathrm{GHz}$ spacing and a very small one corresponding to the first harmonic for a part of the spectrum in the vicinity of the ionization limit. However, the FFT of the structure far below the limit yielded one pronounced peak for the $80-\mathrm{GHz}$ spacing plus four more peaks with decreasing amplitude corresponding to the harmonics. That confirmed that peaks in the structure are narrower if the laser is tuned in the region $\Omega<1$ which means that more microwave field cycles are required to drive atoms to the high-lying states than when the laser is above the ionization limit. To investigate how many cycles it takes, we explicitly measured widths of peaks of Fig. 4.11 and deconvoluted the 10-GHz width due to the laser linewidth using the fact that each peak in the structure can be approximated by a gaussian distribution. An inset of Fig. 4.16 shows one peak of the structure fitted to a gaussian with $R^{2}=0.997$ and a lorentzian with $R^{2}=0.97$. We fitted other peaks in the structure to these distributions and observed that the gaussian distribution describes the experimental peaks better in most cases. As an approximation, we consider all peaks in the spectrum to be gaussian. It is well-known that a convolution of two gaussian peaks is a gaussian again with the width $w_{\text {res }}=\sqrt{w_{1}^{2}+w_{2}^{2}}$. Thus, considering that the laser frequency distribution is also a gaussian, we can easily deconvolute it from the widths of observed peaks. We performed that process for several similar spectra taken at the same field to decrease a statistical error, and the resulting widths for each peak are shown in Fig. 4.16. It is evident that peaks near the ionization limit are about $27 \mathrm{GHz}$ on average, while peaks far below it are much narrower, about $12 \mathrm{GHz}$. At this microwave frequency, each cycle lasts 1/80 ns. Consequently, our data imply that it takes about 2 microwave cycles to drive the atomic 
population to the high-lying states when the laser is tuned near the ionization limit and about 6 cycles when the laser is tuned around $-4000 \mathrm{GHz}$ below the limit.

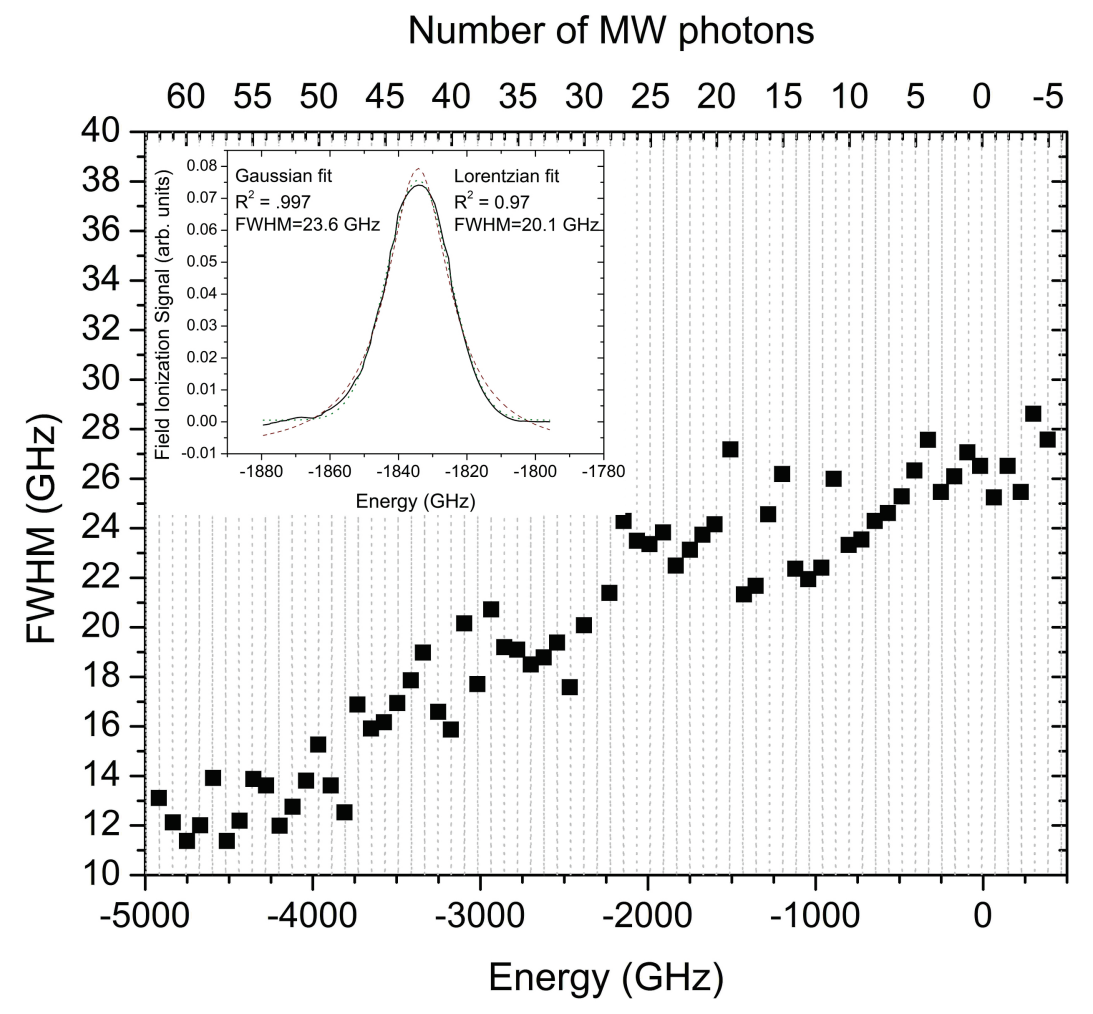

Figure 4.16: FWHM of peaks in the spectrum of the high-lying states as a function of the laser frequency for the $80-\mathrm{GHz}$ microwave field with $170-\mathrm{V} / \mathrm{cm}$ amplitude. Widths of peaks from several different spectra taken at identical conditions were measured. The $10-\mathrm{GHz}$ laser linewidth was deconvoluted from it using the fact that both the laser distribution and peaks can be represented as gaussians. The inset in the graph shows one of the peaks of the spectrum fitted to a gaussian and a lorentzian distribution with corresponding fit parameter and the FWHM. Peaks below $\Omega=1$ are narrower than those above the IL which means that more microwave cycles are required to drive the population to the high-lying states when the laser is tuned in that region.

While the dependence of the widths of observed peaks in the spectrum on the binding energy was expected due to a finite probability of a transition during one microwave cycle, it is not easy to understand why lifetimes of atoms in the high-lying states depend on it. An intuitive notion is that, once atoms are in the high-lying states, they should respond to the external field identically. In other words, it should not matter what the initial binding energy was if the excitation to the high-lying states occurs rapidly, during the first several cycles, as Fig. 4.16 suggests. One possible explanation is that, in fact, when the classical Kepler period is shorter than the duration of the microwave cycle, it takes many more cycles 
to excite population to the limit, and the width of the peaks are broadened due to some other process.

\subsection{Conclusions}

Measuring a spectrum of the extremely high-lying states, we observed a regular structure of peaks starting far below the ionization limit and extending above it, with the extent of the structure depending on the microwave field amplitude. Such spectra are observed for both 38 and 80-GHz microwave fields and different atomic species, Li and Na. Each peak in the structure is spaced an integral number of microwave photons from the ionization limit, and the whole structure equally responds to the depression of the limit by a static field.

An energy extension of the high-lying state structure above the limit was well described by Shuman et al. [38] using a modified Simpleman's model. If the Coulomb potential is included in the model and equals the initial kinetic energy of the electron, a maximum energy transfer between the field and the electron equals $\Delta W=1.5 E \omega^{-2 / 3}$ (Eq. 1.19) which matches our results at $80 \mathrm{GHz}$. There is still ongoing effort in our laboratory to understand and properly describe the observed spectrum near the ionization limit in terms of simple classical picture. Both experimental and numerical work is done towards understanding how the phase between the microwave field and the launching time of the electron affects the observed structure and an entrapment of the population in the the high-lying states. A classical calculation based on a one-dimensional model emphasizes that the energy exchange happens mostly during the first half cycle with a little exchange in the second one. If the phase is right, the electron will lose energy to the microwave field and remain loosely bound in the high-lying states. In the other case, it will gain energy from the field and never return to the core. This classical description seems to work correctly in the vicinity of the ionization limit, but should fail in the region where classical orbiting period is on the order of the microwave cycle. Therefore, it is very surprising that we observe a single structure of peaks that extends far below $\Omega=1$ and above the limit. Nevertheless, it is very likely that we observe two sides of the same phenomenon that has to be described differently. Near 
the ionization limit, an electron can recombine with the ion to form a quasi-stable state by losing energy to the field. Below the ionization limit, on the other hand, atoms are excited to the high-lying states through virtual or real transitions, as is discussed further.

One of the possible mechanisms of such excitation is through real Rydberg states that are adjacent to the excitation laser frequency. Microwave experiments at $15 \mathrm{GHz}$ showed that, at a certain microwave field amplitude, sideband sets of different Rydberg states overlap and form a regular structure of peaks that could, in theory, extend to the ionization limit, driving the population to the high-lying states through a sequence of $n \rightarrow n+1$ transitions. However, the results of the experiment at $80 \mathrm{GHz}$ showed that the structure of peaks that is produced by the high-lying states differs from the one formed by the sidebands of zero-field Rydberg states (we did not observe a regular structure of sideband peaks at $80 \mathrm{GHz}$ ) in number of peaks, their position, and a response to static fields. So, our observations suggest that excitation to the limit, when the laser is tuned in the $\Omega<1$ region, happens not through real transitions. Thus, a different approach must be used. Quite possibly, a quantum description based on multichannel Floquet approach introduced by Giusti-Suzor and Zoller [60] might be applicable in case of low binding energies. In their approach there are multiple channels, each consisting of a Rydberg series and a continuum. Successive channels are displaced in energy by one microwave photon and are coupled by an electric dipole coupling. The Floquet eigenstates are linear superpositions of the wavefunctions of different channels and have both bound and continuum parts. In particular, the eigenstates contain bound states very close to the limit, deeply bound states multiples of the microwave frequency below the limit, and continua multiples of the microwave frequency above the limit. Due to its having a continuum component, an initially bound eigenstate autoionizes into the continuum, just as in the quantum defect theory description of autoionizing states [61].

Another surprising discovery is that atoms in the high-lying states survive the strong microwave field. For example, atoms in $n=250$ are ionized by $75-\mathrm{mV} / \mathrm{cm}$ static fields, 2000 times smaller than the microwave field amplitude we used to observe the high-lying state spectrum at $80 \mathrm{GHz}$. However, atoms in the high-lying states are not affected by the intense field if the electron does not return to the core during a field pulse $[27,57,29,30]$. However, 
in the present case the electron clearly has time to return to the core during the microwave pulse since the Kepler orbital period $T_{K}$ is short compared to the microwave pulse length. At $40 \mathrm{GHz}$ below the limit $T_{K}=3 \mathrm{~ns}$, and at the depressed limit imposed by an $9-\mathrm{mV} / \mathrm{cm}$ field it is $13 \mathrm{~ns}$. Both times are far shorter than the observed 70 and 150-ns decay times observed at 38 and $80-\mathrm{GHz}$ fields. Due to the presence of an electric field, there is also an oscillation in $\ell$, but its period is $\sim 100 \mathrm{~ns}$, long compared to the microwave pulse length, so it has minimal effect. However, the oscillation in $\ell$ or incoherent diffusion of population to higher $\ell$ states may be the source of the long tails seen in the decay curves. Taken as a whole, the data suggest that atoms survive the microwave field because the Rydberg electron spends most of its time far from the core, where the strong microwave field only imposes a ponderomotive quiver on the electron's motion. A 38-GHz field of $90 \mathrm{~V} / \mathrm{cm}$ leads to a quiver amplitude of $400 \mathrm{a}_{0}$, small compared to the $120,000 \mathrm{a}_{0}$ orbital size of an atom bound by $38 \mathrm{GHz}$. When the electron does come to the core it has an approximately equal chance of gaining or losing energy, so it is reasonable to expect atoms to survive several field cycles, as observed. Essentially the same result was observed in calculations to elucidate the source of superponderomotive electrons [58]; they are observed at the intensities which bring states just below the limit into multiphoton resonance with the ground state. In the usual case of ionization by a pulse of microwaves or laser light, the ponderomotive shift, that is larger than the frequency of the field, due to the changing intensity during the pulse leads to resonant excitation of the high-lying states, as shown by the calculations of Muller [58]. 


\section{Chapter 5}

\section{Production of extremely high-lying states by strong microwave fields}

\subsection{Introduction}

Here we report a surprising discovery that, for a wide range of initial states, a substantial fraction, $\sim 5-15 \%$, of the atoms is found in high-lying Rydberg states subsequent to a 200 ns long microwave pulse. Previously, atoms have been detected in high-lying states subsequent to exposure to short microwave pulses containing only a few field cycles [44] and trains of up to 40 unipolar pulses with a vanishing time average field [41]. However, they have only been observed with long pulses when starting from very high-lying states [23]. They have not been observed before in microwave ionization of low lying states.

Ionization of ground state atoms by intense laser pulses in both the tunneling and multiphoton regimes can also result in the production of highly excited, or Rydberg, atoms as a byproduct. An example of the former is the ionization of He by 1-GV/cm 800-nm pulses 30 fs long. It results in $10 \%$ of the atoms' being left in states of principal quantum number $n$ between 7 and 15, a rescattering process dubbed "frustrated tunnel ionization" [36, 37]. In the multiphoton regime, with somewhat longer pulses, population is also observed to be left in the Rydberg states, but in this case it is attributed to multiphoton excitation brought into resonance by the AC Stark shift during the laser pulse $[8,27]$. Our measurements of laser excitation in the presence of the microwave field suggest that the high-lying states are produced by resonant excitation, as in laser ionization in the multiphoton regime [62]. In 
earlier experiments the production of highly excited states in the recombination of electrons with ions induced by half cycle pulses and microwave fields has been reported [38, 42, 43]. These are essentially rescattering processes, rather similar to frustrated tunnel ionization. In sum, with respect to the production of highly excited states, Rydberg atoms in microwave fields are similar to ground state atoms in strong laser fields. The notion that Rydberg atoms and microwaves constitute a good system for the study of strong field processes is not new; the first microwave ionization experiments were undertaken to connect tunneling and multiphoton ionization [23].

How atoms survive an intense field was first addressed in calculations of high frequency stabilization $[63,64]$. More recent calculations to elucidate the source of superponderomotive electrons in ATI are, however, more relevant to this problem [58]. Superponderomotive electrons are those with energies in excess of the prediction of the Simpleman's Model $[40,65,39]$. They are formed at the intensities at which the AC Stark shift from the laser field brings states just below the limit into multiphoton resonance with the ground state. At these intensities quasi stable states are produced in which the atom survives multiple recollisions with the core, with some loss of population on each recollision [58].

We conducted experiments at 17 and $38 \mathrm{GHz}$ fields that showed that the production of the high-lying states is a general phenomenon inherent in the microwave ionization of Rydberg atoms and occurs for any Rydberg state that starts ionizing in the microwave field. However, similar measurements taken at $80 \mathrm{GHz}$ clarified that the high-lying states are only produced when the initial state is displaced by an integral number of photons from the energy region just below the ionization limit. Atoms are driven to the limit by a multiphoton transition when the initial state can be ponderomotively shifted in resonance with the limit. Essentially the same phenomenon was observed in the calculation by $\mathrm{H}$. Muller [58] that explained a flat plateau in the ATI spectrum. 


\subsection{Production of extremely high-lying states by $38-\mathrm{GHz}$ mi- crowave field in $\mathrm{Li}$}

\subsubsection{Experimental Approach}

An experimental apparatus used in this experiment is identical to the one described in section 3.3.1. In short, a thermal beam of $\mathrm{Li}$ atoms passes through the antinode at the center of a 38.34-GHz Fabry-Perot cavity where the atoms are excited to a Rydberg state by three $20-n s$ dye laser pulses via $2 s \rightarrow 2 p \rightarrow 3 s \rightarrow n p$ transitions. The excited atoms are then exposed to a microwave field pulse, as shown in the timing diagram in Fig. 3.7. After the end of the microwave pulse, typically $300 \mathrm{~ns}$ later, atoms are field ionized by a slowly rising FIP. The electrons ejected by the ionization field are detected by a dual micro-channel plate detector. The time resolved MCP signal tells us how many atoms were not ionized by the microwave pulse as well as their final state distribution. The MCP signal is captured by a gated integrator or oscilloscope and recorded for later analysis. The integration gate can be set up to detect only signal from selected states.

The microwave cavity consists of two brass mirrors $40.5 \mathrm{~mm}$ in diameter with $75.9-\mathrm{mm}$ radii of curvature. The on-axis spacing between mirrors is $44.5 \mathrm{~mm}$. The cavity is operated on the $\mathrm{TEM}_{012}$ mode at a frequency of $38.34 \mathrm{GHz}$ with $Q=5100$. The microwave system generates a $38.34-\mathrm{GHz}$ pulse with 0 - to $90 \mathrm{~V} / \mathrm{cm}$ amplitude and a variable width. All apparatus is triggered at a $500 \mathrm{~Hz}$ repetition rate by a frequency-doubled Nd:YLF laser. The linewidth of the laser making the last transition is about $8 \mathrm{GHz}$, and its frequency can be tuned to populate any Rydberg state of $n>35$. The three laser beams are sent to the vacuum chamber and focused to less than 1-mm diameter spots where they cross each other and the atomic beam. The laser field and microwave field are polarized vertically throughout the experiment.

\subsubsection{Experimental Observations}

An initial objective of this experiment was to study ionization thresholds of Li Rydberg states in a microwave field of $38 \mathrm{GHz}$ for a wide range of initial binding energies. Ionization 
problems at this frequency were discussed in detail in Chapter 3, and Fig. 3.11 showed the final state distributions of atoms that survive the microwave fields vs laser tuning. An unexpected discovery was an evidence of the extremely high-lying states that were produced by the $38-\mathrm{GHz}$ microwave field for all initial states. The signal from these states is seen at ionizing fields between 0 and $2 \mathrm{~V} / \mathrm{cm}$ (times between 10 and $50 \mathrm{~ns}$ ) in Fig. 3.11. Both 36$\mathrm{V} / \mathrm{cm}$ and $90-\mathrm{V} / \mathrm{cm}$ scans in Fig. 3.11 show the presence of extremely high-lying states for initial energies down to $-2400 \mathrm{GHz}$ for a $36-\mathrm{V} / \mathrm{cm}$ field, and $-3300 \mathrm{GHz}$ for the $90-\mathrm{V} / \mathrm{cm}$ field. Especially surprising is the $90 \mathrm{~V} / \mathrm{cm}$ scan, as we see that almost all other population is ionized, but weakly bound states are still present.
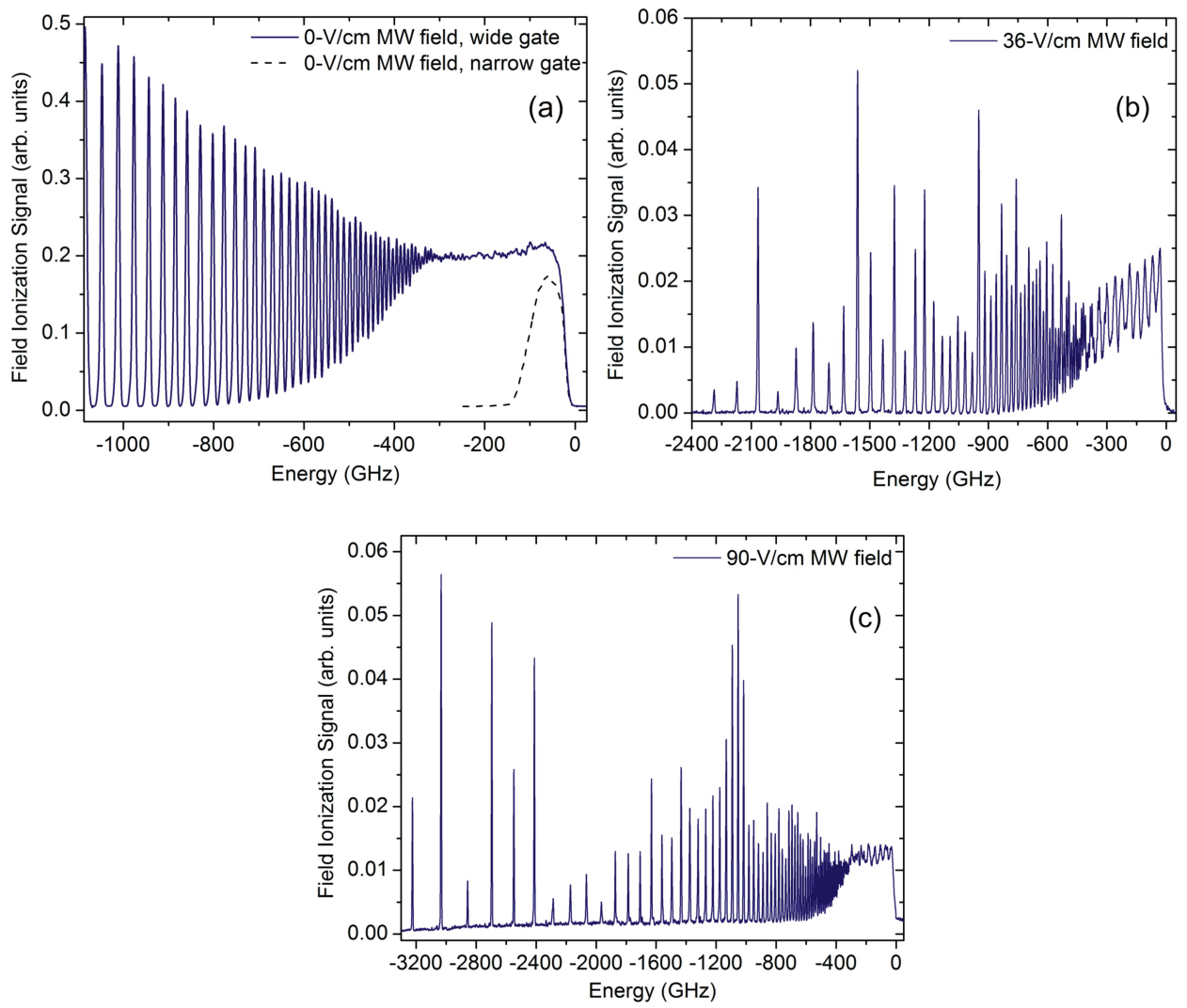

Figure 5.1: Field ionization signal as a function of energy for three microwave field amplitudes. (a) 0 $\mathrm{V} / \mathrm{cm}$, in which case two different gates were used, a wide gate to accept the signal from all Rydberg states of $n \geq 37$ (solid line) and a 50-ns gate to detect only atoms within $75 \mathrm{GHz}$ of the IL (dashed line); (b) 36 $\mathrm{V} / \mathrm{cm}$, a spectrum recorded with the 50-ns gate; (c) $90 \mathrm{~V} / \mathrm{cm}$, a spectrum recorded with the 50 -ns gate. In (b) and (c) we see non-zero signal within the narrow gate, when the laser is tuned to the Rydberg states from which no signal is detected with the same gate when the MW field is off. Not only the expected microwave structure is present near the IL, but also a large fraction of population is observed due to a transfer from the bound Rydberg states to the extremely high-lying states, even when the laser is tuned to a state with $n$ as low as 30 . 
The final state distributions shown in Fig. 3.11 imply that bound atoms detected subsequent to the microwave pulse, as shown in Fig. 3.8, are largely in highly excited states. This suggestion is confirmed in the following way. Simultaneously with the oscilloscope traces of Fig. 3.11, the field ionization signal was recorded using a 50-ns gate, positioned to detect only the extremely high-lying states, those within $75 \mathrm{GHz}$ of the depressed ionization limit of -25 GHz. Fig. 5.1 shows the corresponding spectra. First, where the initial states can not be resolved by the laser, close to the limit, there is obvious structure at the microwave frequency, as shown in Fig. 3.8 (c). It is particularly apparent in Fig. 5.1 (b) in which peaks in the number of surviving atoms are observed at integral multiples of the microwave frequency below the depressed ionization limit. Second, we see a peak in the population in the extremely high-lying states when the laser is tuned near $\Omega=1(n=56$, $-1050 \mathrm{GHz}$ ). Fig. 5.1 suggests that this peak is probably due to the fact that atoms are retained longer in the initial states for this laser tuning. Third, the population transferred to high-lying states from the $n=41$ state at $-1960 \mathrm{GHz}$ is much lower that from the adjacent states, making this state stand apart from its neighbors. This particular state is also more resistant to ionization, as can be clearly seen in Fig. 3.9 (a). In this case the population remains in low lying states.

Fig. 5.1 points to the surprising fact that not all the atoms are ionized by strong microwave fields. Instead, they are found in highly excited states. This point is made explicitly by measuring the fraction of atoms surviving the microwave pulse as a function of microwave field amplitude for several laser frequencies. Fig. 5.2 shows the total survival probability when the integration gate is wide, allowing signal from all bound states to be recorded. Almost all the population loss, due to ionization, occurs at low microwave fields. For fields above $50 \mathrm{~V} / \mathrm{cm}$ there is minimal population loss with increasing field. The surviving bound population is found entirely in the highly excited states, as shown by Fig. 5.3 , which shows the result of the same measurement taken with the narrow 50-ns gate, to collect only the signal due to the extremely high-lying states. For initial energies lower than $-700 \mathrm{GHz}$ population begins to be observed in the high-lying states when $50 \%$ of the atoms are ionized. For states initially bound by less than $700 \mathrm{GHz}$, population is found in high- 


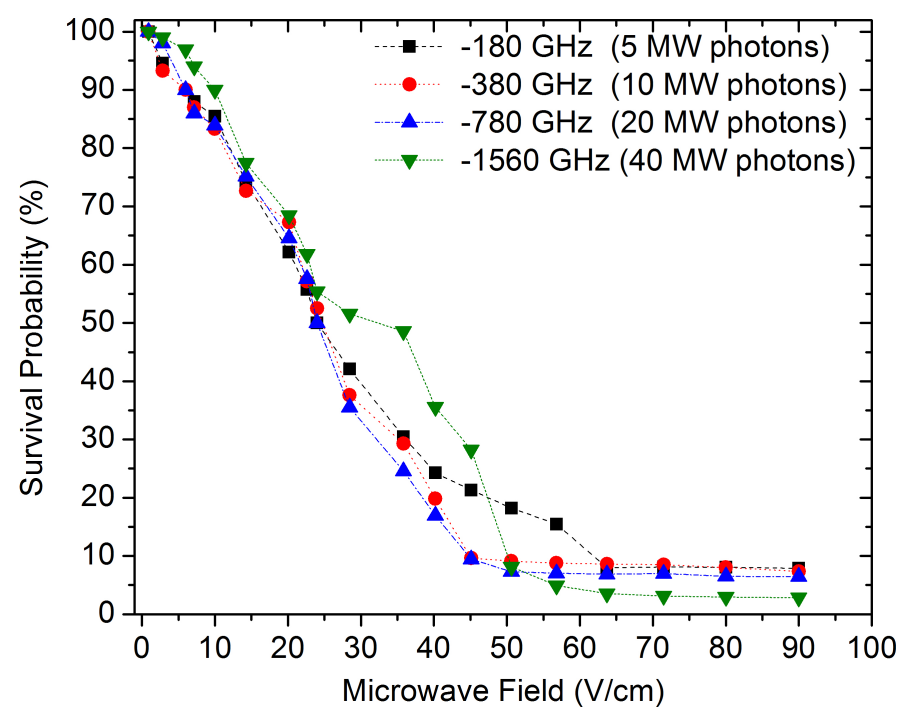

Figure 5.2: Fraction of atoms surviving the MW field as a function of its strength when the laser frequency is tuned to four different Rydberg states $(n=46,65,93$, and 135). All bound states are detected (wide gate). For each of these binding energies the $50 \%$ ionization field is almost the same, as predicted by Eq. 1.16. For all of the states minimal further ionization is produced by raising the microwave field above 60 $\mathrm{V} / \mathrm{cm}$.

lying states at microwave fields less than the $50 \%$ ionization field. Finally, the population in these states decays very slowly as the microwave pulse length is increased; we measured the lifetime of the trapped population in the field to be about $50 \mathrm{~ns}$ in a $90-\mathrm{V} / \mathrm{cm}$ field and $120 \mathrm{~ns}$ in a $45-\mathrm{V} / \mathrm{cm}$ field when the atoms are initially bound by 10 microwave photons. Fig. 5.4 explicitly shows decay curves of atoms in the high-lying states when the laser is tuned 40 microwave $(n=46)$ photons below the ionization limit for different microwave field amplitudes. The observed curves exhibit double exponent decay at higher fields and are fit by a fast decaying exponent plus a constant. However, at lower fields a probability to produce the high-lying states in one cycle is very small, and more cycles are required to produce a sufficient number of atoms in the high-lying states before the population starts decaying very slowly.

To understand more clearly the ionization, redistribution, and trapping of the initial population by the microwaves, we have measured final state distributions as a function of the microwave field amplitude. Again we recorded the oscilloscope traces as the microwave field was varied with the laser frequency fixed at energies of $-192,-386,-770$, and -1530 GHz. These traces were mapped to a gray scale as seen in Fig. 5.5. With an initial binding 


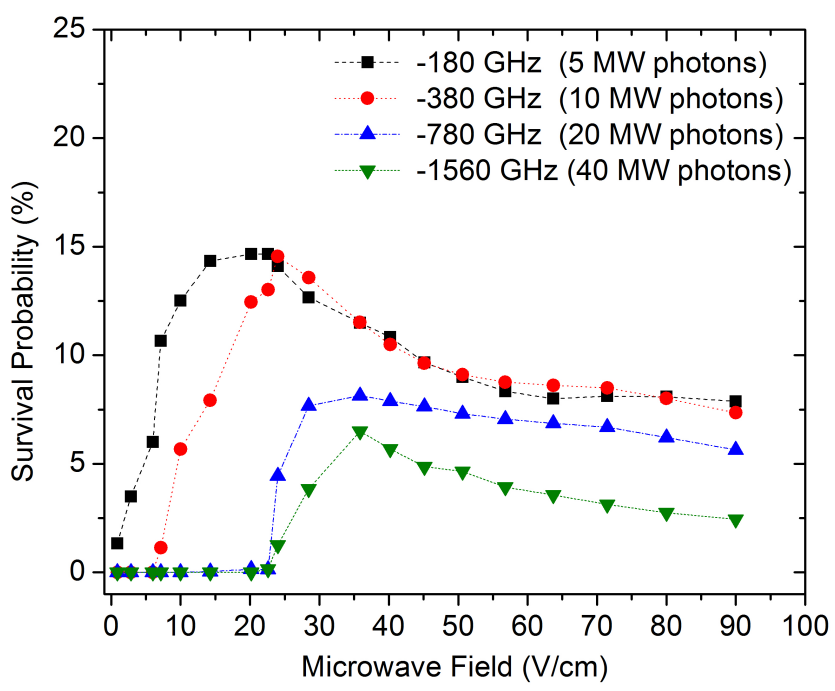

Figure 5.3: Fraction of atoms surviving the microwave field as a function of its strength when the laser frequency is tuned to four different Rydberg states, the same states as shown in Fig. 5.2, $n=46,65,93$, and 135. Only the extremely high-lying states are detected (narrow 50-ns gate). The field ionization signals at microwave fields above $60 \mathrm{~V} / \mathrm{cm}$ in Fig. 5.2 are due to atoms being left in high-lying states.

energy of $192 \mathrm{GHz}$ atoms are either transferred to highly excited states, which are ionized at a field of $\approx 2 \mathrm{~V} / \mathrm{cm}$ (a time of $40 \mathrm{~ns}$ ), or ionized. With an initial binding energy of $192 \mathrm{GHz}$ atoms are not completely removed from the initial state until a microwave field in excess of $30 \mathrm{~V} / \mathrm{cm}$ is reached. However, population begins to appear in the highly excited states at microwave fields below $10 \mathrm{~V} / \mathrm{cm}$. An initial binding energy of $386 \mathrm{GHz}$ leads to similar results in that there is a field regime in which atoms are observed at both the initial binding energy, at times near $80 \mathrm{~ns}$, and in highly excited states, at a time near $40 \mathrm{~ns}$. There is, however, some population observed between the initially excited state and the ionization limit. At binding energies of $386 \mathrm{GHz}$ or less, large numbers of atoms are observed in highlying states at fields below the $50 \%$ ionization fields. When the binding energy is increased to $770 \mathrm{GHz}$, it is no longer the case that atoms are found in deeply bound states and highly excited states over a range of microwave fields. The atoms are either deeply bound near $\Omega=1$ or in highly excited states, with few atoms between these energies. At microwave fields between 10 and $30 \mathrm{~V} / \mathrm{cm}$ it appears that population is transferred to more deeply bound states near $\Omega=1$ where stable classical orbits exist in the microwave field. Higher field amplitudes lead to ionization. Finally, when the initial binding energy is $1530 \mathrm{GHz}$ it 


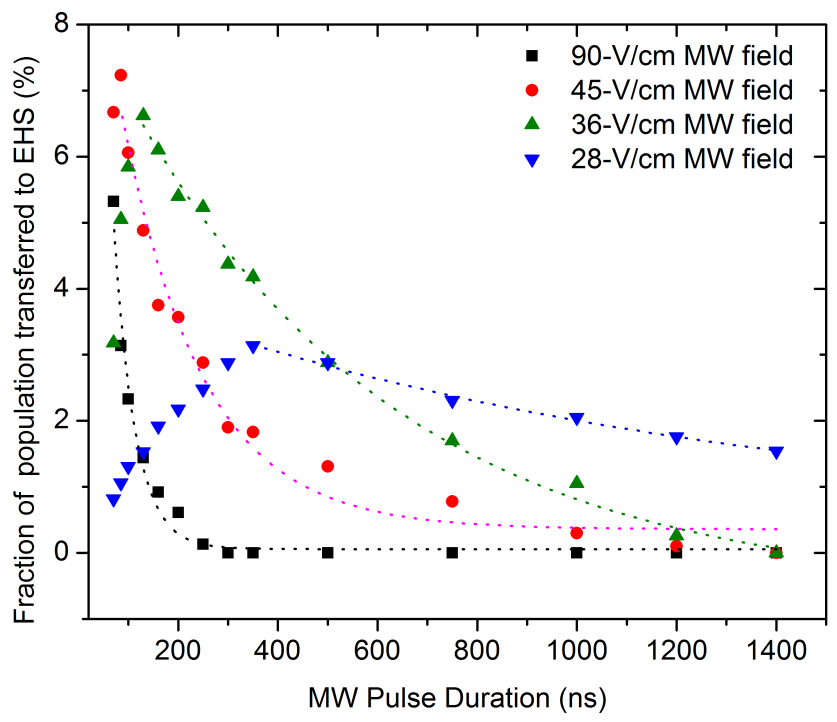

Figure 5.4: Fraction of atoms in the high-lying states surviving the microwave field as a function of the width of the microwave pulse for several field amplitudes. The laser is tuned 40 microwave photons below the IL $(n=46)$, but only the extremely high-lying states are detected (narrow 50-ns gate). 90 and $45-\mathrm{V} / \mathrm{cm}$ curves are fit to $A e^{-x / t}+B$. Lower field curves show that many microwave cycles are required to bring atoms to the limit when the laser is tuned far from it.

appears that microwave fields of 20 and $30 \mathrm{~V} / \mathrm{cm}$ transfer population to states in which the microwave frequency is half and equal the Kepler frequency, respectively. Above $30 \mathrm{~V} / \mathrm{cm}$ only highly excited states are observed.

With the exception of initial states within $400 \mathrm{GHz}$ of the ionization limit, we observe that highly excited states begin to appear around fields at which $50 \%$ ionization occurs. In other words, the production of highly excited states might be related to microwave ionization. It is suggested by the Simpleman's model [39, 40,65] which predicts that electrons ionized at the peak of the microwave field have zero drift velocity, only an oscillation in the field, so the electron remains near the ion core, oscillating in the field. The Simpleman's model must be modified to take into account the Coulomb potential [38], but the basic idea is unchanged; microwave ionization at some phase of the microwave field produces electrons with zero time average velocity. These electrons therefore remain in the vicinity of the ion core and are in some sense bound. In quantum mechanical terms they are quasi-stable atom-field, or Floquet states [60]. In either case, when the microwave field is turned off over many field cycles, the atom is left in a highly excited state.

If the production of highly excited states is an inherent part of microwave ionization, 

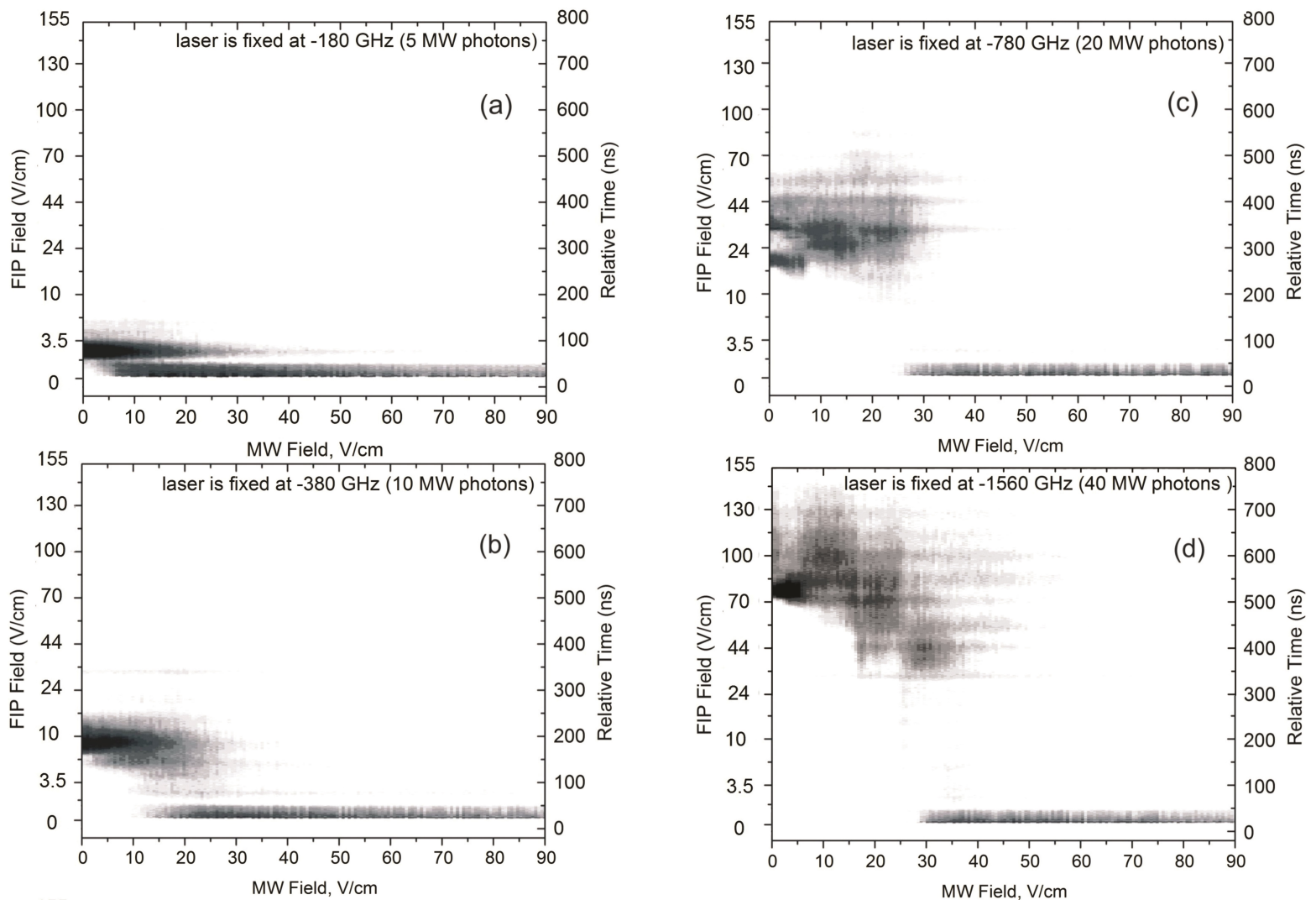

Figure 5.5: Final state distribution as a function of the MW field amplitude when the laser frequency is tuned to four different Rydberg states. The distributions are gray scale representations of time resolved field ionization signals. For convenience the vertical scale is shown both as time and field in the FIP. The FIP starts at 20 ns. Population is always found in extremely high-lying states at fields when a large fraction of atoms is ionized. Redistribution of the population at lower microwave fields occurs, but atoms are in no case continuously distributed between the initial state and the IL.

why were they not observed long ago? Experiments with H were reported in 1974, and the first alkali experiments in 1983 [14]. The most probable reason is that the early microwave ionization experiments were done under conditions in which there were static fields, which destroy the highly excited states. The initial alkali experiments were done in waveguide cavities in which the atoms were $1 \mathrm{~mm}$ from a metal septum, with stray fields of at least $100 \mathrm{mV} / \mathrm{cm}$. In later $\mathrm{H}$ experiments static fields depressed the ionization limit to $n=114$, corresponding to a field of $3.4 \mathrm{~V} / \mathrm{cm}$ [66]. In these experiments the amplitudes of the microwave fields were often in excess of $100 \mathrm{~V} / \mathrm{cm}$, and stray fields of $100 \mathrm{mV} / \mathrm{cm}$ were not thought to be important. Finally, most microwave ionization experiments were done at lower frequencies, which allow stray fields a longer time to destabilize a synchronized orbit 
during each field cycle. In the following, we explicitly check these notions by revisiting microwave ionization below $20 \mathrm{GHz}$.

\subsection{Production of extremely high-lying states of Li and $\mathrm{Na}$ by $17-\mathrm{GHz}$ fields}

Microwave ionization of Li by $38-\mathrm{GHz}$ fields has shown that roughly $10 \%$ of the atoms are left in highly excited states within one or two microwave photons of the ionization limit when microwave ionization occurs as discussed in the previous section. Until these measurements, there was only one measurement which showed the production of high-lying states, but in that experiment atoms initially in states of $n \cong 80$ were exposed to a five cycle $11.5 \mathrm{GHz}$ pulse, which resulted in as much as $35 \%$ of the population's being left in states of $n>120$ [44]. In all other experiments, with microwave pulses thousands of field cycles long, after the microwave pulse population was apparently either in the initial state or ionized [14]. No population was found in intermediate states. Why are the recent $38-\mathrm{GHz}$ Li results so different from the previous results? Is it a question of experimental technique, something special about $\mathrm{Li}$, or the relatively high frequency of $38 \mathrm{GHz}$ ? Below we report the ionization of $\mathrm{Li}$ and $\mathrm{Na}$ by a $16.9 \mathrm{GHz}$ microwave field to address this question. As we shall show, the way the previous experiments were done would have precluded leaving atoms in high-lying states or detecting them. Furthermore, there is nothing special about $38 \mathrm{GHz}$ or Li. Atoms are left in the high-lying states when both $\mathrm{Li}$ and $\mathrm{Na}$ are ionized by a $16.91 \mathrm{GHz}$ field. In the sections which follow we outline the experimental technique, present our results, and discuss the conclusions which can be drawn from them.

\subsubsection{Experimental Approach}

The apparatus used for this experiment is similar to the one discussed in section 3.2.1. A thermal beam of ground state lithium or sodium atoms crosses the electric field antinode at the center of a 16.91-GHz Fabry-Perot microwave cavity. There atoms are excited to a Rydberg state by a sequence of transitions induced by 20-ns dye laser pulses. Following 
laser excitation, atoms are subjected to a microwave pulse, typically 200-ns long, as shown in the timing diagram of Fig. 3.1. About 300 ns later atoms are exposed to a $1-\mu$ s rise time FIP. Depending on the polarity of the FIP, either electrons or ions are sent upwards to a dual microchannel plate detector. The MCP signal is amplified, captured by a gated integrator or oscilloscope, and recorded in a computer for later analysis. The integration gate or amplitude of the FIP can be chosen to detect signal only from the high-lying states with $n>250$. The minimum value of the static field is about $5 \mathrm{mV} / \mathrm{cm}$ and can be increased in a control fashion.

Li atoms are excited via the $2 s \rightarrow 2 p \rightarrow 3 s \rightarrow n p$ transitions. The linewidth of the last laser is about $8 \mathrm{GHz}$, and its frequency can be continuously tuned to populate any Rydberg state of $n>20$. Na atoms are excited via $3 s \rightarrow 3 p \rightarrow 3 d \rightarrow n f$ transitions. The linewidth of the last laser is about $12 \mathrm{GHz}$. The dye laser beams are sent to the vacuum chamber and focused to less than 1-mm diameter spots where they cross each other and the atomic beam. Unless stated otherwise, the laser field and microwave field are polarized vertically throughout the experiment.

\subsubsection{Experimental Observations}

A major objective of the current work was to determine if the population trapping in the extremely high-lying states observed in $38.34 \mathrm{GHz}$ microwave ionization of $\mathrm{Li}$ atoms also occurs in 16.91-GHz microwave ionization [57]. To detect atoms in these states, we use a negative FIP, narrow the integration gate to $50-\mathrm{ns}$, and position the gate in such a way that only signal from states that lie within $50 \mathrm{GHz}$ of the ionization limit is recorded. We can scan the frequency of the $3 s-n p$ dye laser to obtain the spectrum of high-lying Li Rydberg states that remain bound after the microwave pulse. The spectrum obtained with a 300$\mathrm{V} / \mathrm{cm}$ microwave field is presented in Fig. 5.6. There is no signal above the limit, denoted by zero on the horizontal axis, because all photoionized electrons have time to leave before the FIP commences and are not detected. We denote the middle of the down slope of this signal as the ionization limit depressed by the static field $E_{s}$ present during the excitation. Explicitly, the limit is depressed by $\Delta W=2 \sqrt{E_{s}}$. In Fig. 5.6 the depressed ionization limit 


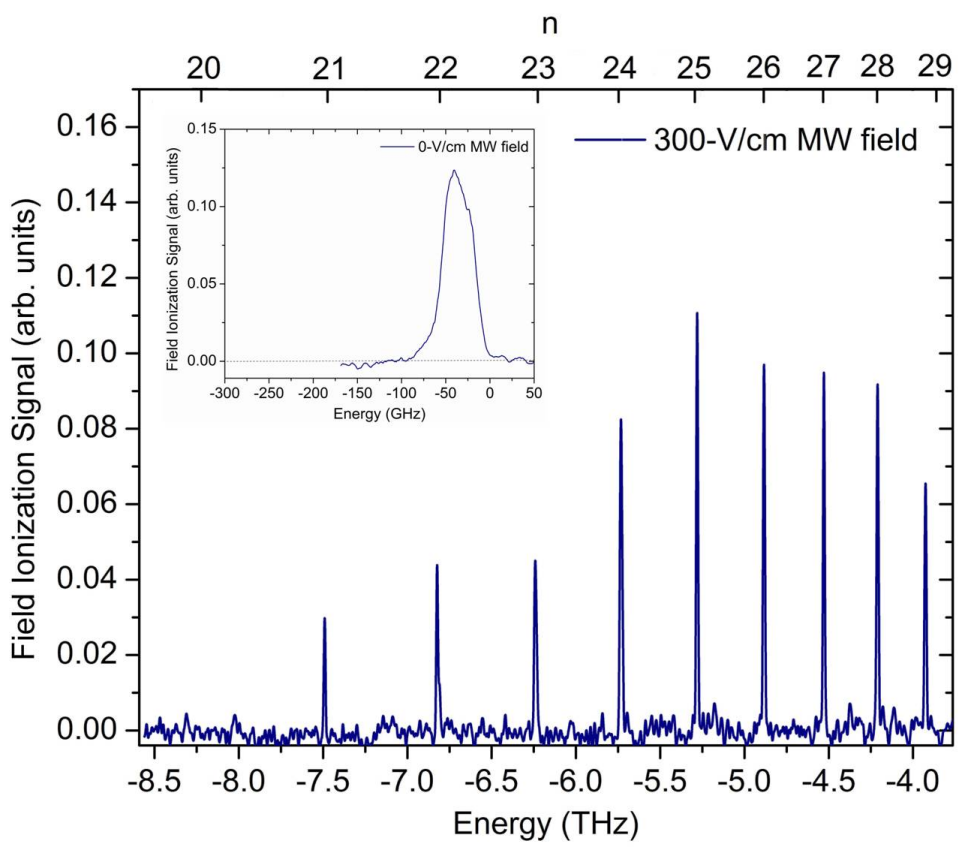

Figure 5.6: Field ionization signal from the high-lying states of Li after exposure to a 300-V/cm microwave pulse as a function of the laser frequency, in terms of energy relative to the ionization limit. The inset shows the spectrum in the vicinity of the ionization limit obtained with the same narrow integration gate when no MW field is present. If the ionization spectrum is measured under identical circumstances (detecting ions produced by the mw pulse), a similar trace is obtained. In other words, we detect the high-lying states when the laser is tuned to any Rydberg state that can be ionized by the microwave field.

is at about $-15 \mathrm{GHz}$, which corresponds to a $6.6-\mathrm{mV} / \mathrm{cm}$ static field. Fig. 5.6 demonstrates that with a $300-\mathrm{V} / \mathrm{cm}$ microwave field, we observe the high-lying states for initial states of $n \geq 21$, which is the lowest $n$ state we can ionize with a $300 \mathrm{~V} / \mathrm{cm}$ microwave field. To verify that we only detect high-lying states, we show in the inset of Fig. 5.6 a spectrum taken with the microwave field off. The signal is non-zero only very close to the ionization limit, from weakly bound states with $n>250$. Although the width of the peak, $50 \mathrm{GHz}$, is more than five times as wide as our laser linewidth, further reduction of the amplitude of the FIP or the gate width does not lead to a narrower peak, only one diminished in amplitude. We believe that reduced collection efficiency for electrons produced by a small FIP requires a FIP of amplitude large enough to ionize states of $n>250$. Using a positive FIP we can detect a microwave ionization signal for $n \geq 21$. It is worth mentioning here that with the negative FIP we detect zero signal when the laser frequency is scanned over the ionization limit when the microwave field is on. Thus, electrons are not spatially trapped in a plasma 

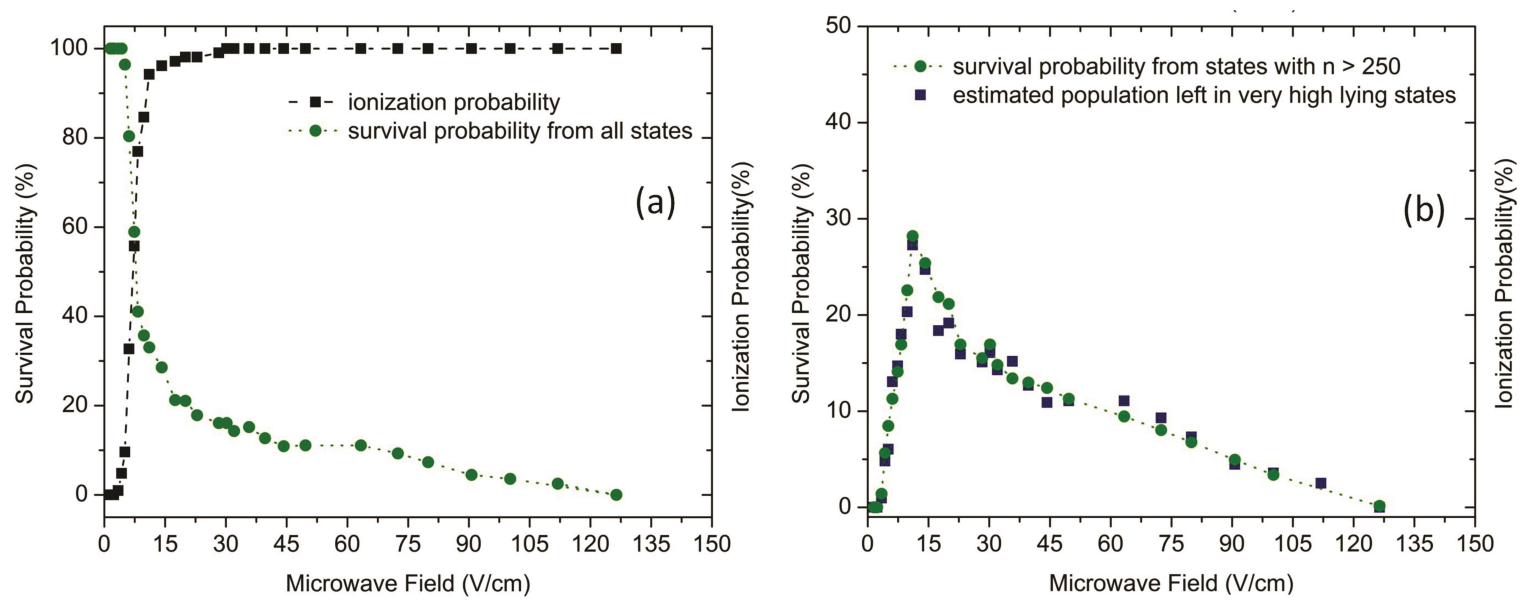

Figure 5.7: Microwave ionization of the Li $73 p$ state.(a) Fraction of atoms surviving the MW field as a function of its strength detecting electrons from all bound states of $n \geq 55$ (circles) and fraction of atoms apparently ionized by the MW field as a function of its strength observed by detecting ions with the positive FIP (squares). The apparent microwave ionization signal collected with the positive FIP is due to both microwave ionization and field ionization of atoms in state of $n \geq 90$. Because the high-lying states are present in both signals, they are not complementary. (b) Fraction of atoms detected in the high-lying, $n \geq 250$, subsequent to the microwave pulse. Fraction of atoms in such high-lying states is also estimated from graph (a) as: ionization probability minus (100\% minus survival probability) for any given MW power (squares). When the MW field exceeds $30 \mathrm{~V} / \mathrm{cm}$, the only bound population remains is in states with $n>250$.

by ions in the interaction region before the arrival of the FIP, and what we detect is indeed a population left bound in the extremely high-lying states when a Rydberg state is exposed to a strong microwave field $[67,68]$. We have, in addition, checked that the observed highlying state signals are not dependent on the number of Rydberg atoms excited, ruling out collisional effects which might lead to the production of high-lying states.

To determine the fraction of atoms excited to the extremely high-lying states, we tune the laser to a specific Rydberg state, and, using a negative FIP and electron detection, record the electron signal as a function of the microwave field amplitude. We use a narrow 50-ns gate to measure the population left in the high-lying states and a wider gate to detect surviving atoms in all detectable $(n \geq 60)$ Rydberg states. To compare our results to the previous microwave ionization experiments, we, as well, detect ions with a positive FIP, the amplitude of which is not high enough to field ionize the initial state, but ionizes atoms with $n>90$ as was discussed in detail in section 3.2.1. We recorded such data for several Rydberg states, and show a characteristic plot in Fig. 5.7 for the $n=73 \mathrm{Li}$ Rydberg state. As was 
noted before, the two detection techniques do not yield complementary signals because of the presence of the high-lying states in both. This point is explicitly made by Fig. 5.7 (b) in which we show the electron signals from the high-lying states measured with the narrow gate. All the negative FIP signal at microwave fields over $30 \mathrm{~V} / \mathrm{cm}$ in Fig. 5.7 (a) is due to high-lying states. Fig. 5.7 shows that atoms exposed to the microwave pulse are left in the initial state or nearby states, in the very high-lying states, or ionized. Most important, we observe a substantial population transfer to states with $n>250$. For $n=73$ it is nearly $30 \%$ at the microwave field just above the threshold for ionization, and it decreases as the microwave field is increased.

A striking result of this experiment is the dependence of the production of the high-lying states on the static field, as shown in Fig. 5.8 for two different Rydberg states of Li, $24 p$ and $58 \mathrm{p}$. We use a narrow 50-ns gate to detect only the high-lying states and record the signal while continuously scanning the bias voltage on one or two plates. An example of such measurement is presented in the inset of Fig. 5.8 for $n=58$ with a microwave field of $6 \mathrm{~V} / \mathrm{cm}$, where the production of the high-lying states is at a maximum. For each of the bias voltages, we determine the field in the interaction region by measuring the depression of the ionization limit. Since we are never able to eliminate completely the depression of the limit, we believe there to be a time dependent stray field of approximately $6.6 \mathrm{mV} / \mathrm{cm}$. Converting the bias voltages into fields, we construct the static field dependence for both initial states, as shown in Fig. 5.8. In both cases the microwave field is set just above the threshold field, where the production of e high-lying states is maximized. Fig. 5.8 shows that the $n=24$ and 58 curves exhibit the same behavior: the maximum high $n$ signal occurs at the lowest static field, and we detect no signal when the field is increased to approximately $35 \mathrm{mV} / \mathrm{cm}$. The $35-\mathrm{mV} / \mathrm{cm}$ static field is large enough to ionize all the population in the high-lying states of $n>360$. Returning to the Fig. 5.8, we notice that the middle of the curves nearly coincide, so, all in all, it's impossible to say which curve belongs to which state just by looking at the graph. That suggests that there is no dependence on the initial state, as long as the microwave field is strong enough for ionization. The decrease of population in the high-lying states with increasing static field is most likely due 


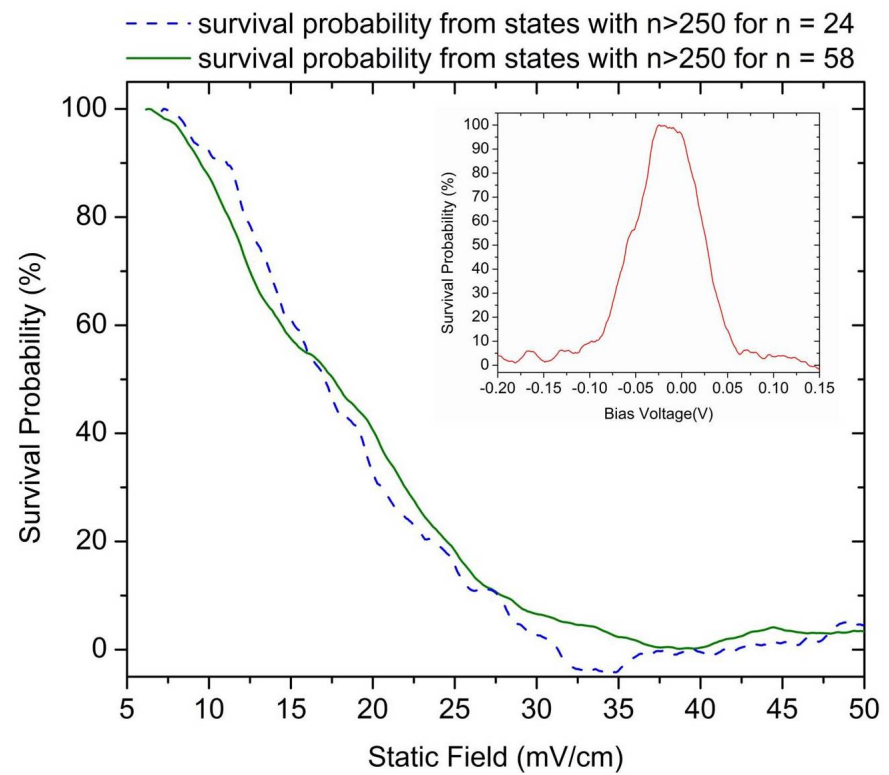

Figure 5.8: Relative number of atoms in the high-lying states with $n>250$ after the MW pulse as a function of static field in the interaction region. The laser frequency is tuned to two states: $\mathrm{n}=58$ and 24 . The inset shows a relative number of atoms in the high-lying states as a function of bias voltage on one of the plates after excitation of the Li $58 p$ state and exposure to a $6-\mathrm{V} / \mathrm{cm}$ microwave pulse. The main figure show the relative numbers of surviving high-lying atoms as a function of the static field for the Li $24 p(-)$ and $58 p$ states. The microwave fields are 215 and $6 \mathrm{~V} / \mathrm{cm}$, respectively. In both cases these microwave fields are just above the ionization threshold fields. The two curves are almost identical, and for both states no bound atoms are detected when the static field is about $35 \mathrm{mV} / \mathrm{cm}$, a field that ionizes states as low as $\mathrm{n}=$ 360 .

to either of two effects: the non-zero static field's destabilizing the electron's orbit in the microwave field, or the static field is directly ionizing all population in these states. To show that the latter is the dominant effect, we minimize the static field and introduce a 100-ns square pulse of small amplitude that starts after the microwave pulse and ends before the FIP. Using several values of amplitude of the square pulse, we detected no difference in the survival probability of the high-lying states compared to a corresponding measurement in dc static field. In short, the dependence is essentially that shown in Fig. 5.8. A similar result has been reported by Zhao et al. [41], who showed that exposure to unipolar pulse trains biased to produce zero time average field leads to minimal ionization while an non zero time average field leads to rapid ionization. The $35-\mathrm{mV} / \mathrm{cm}$ static field is strong enough to ionize population within $\sim 32 \mathrm{GHz}$, or two microwave photons, of the ionization limit according to $\Delta W=2 \sqrt{E_{s}}$. Hence, we can infer that the observed high-lying states are states with $n>360$. 


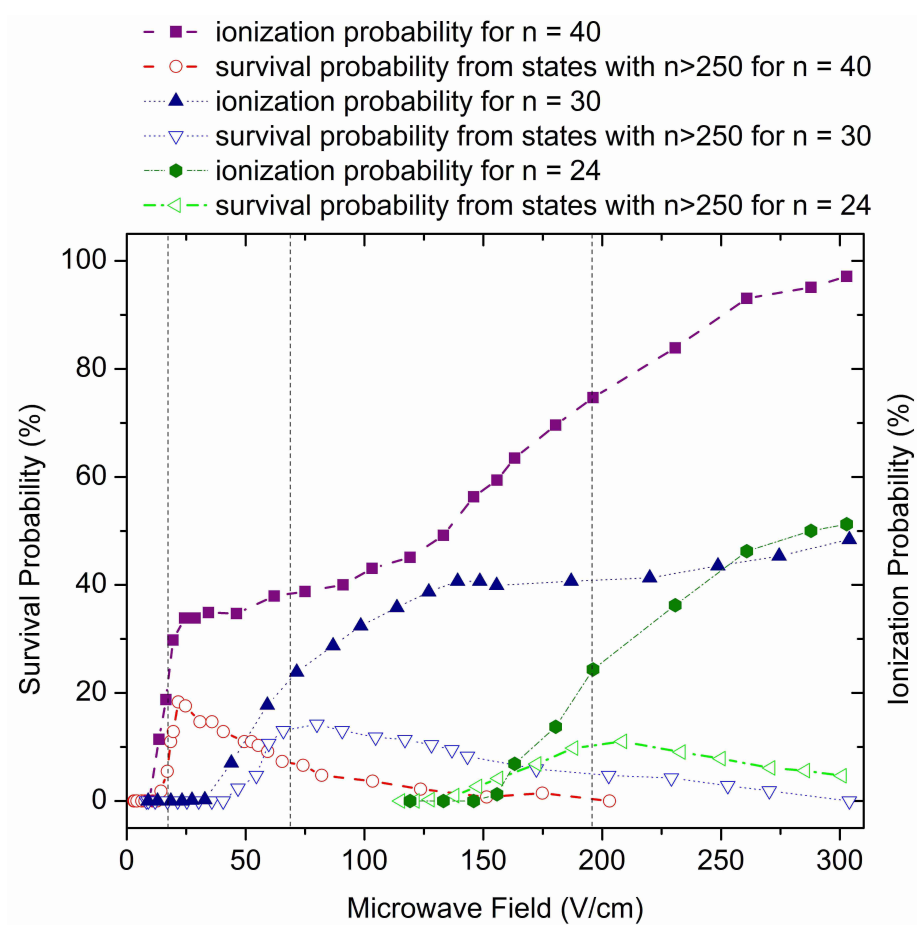

Figure 5.9: Fractional apparent ionization for the Na $24 f$ (filled circle), $30 f$ (filled triangle), and $40 f$ (filled square) states obtained with a positive FIP. The signals contain both microwave ionization and field ionization of high-lying states with $n>250$. Vertical lines correspond to the $50 \%$ ionization threshold of $|m|=0$ and 1 states measured by Pillet et al. [13] Fractional high-lying state signals obtained for the $24 f$ (open circles), $30 f$ (open triangles), and $40 f$ states (open squares) obtained with a negative FIP and a narrow integration gate. As in $\mathrm{Li}$, the high-lying states begin to be observed at the field at which microwave ionization starts, and in all cases roughly $15-20 \%$ of the atoms are left in the high-lying states.

Another important objective was to show that the excitation to the extremely highlying states is a general phenomenon inherent to all Rydberg atoms and is not peculiar to lithium. To do so, we have repeated the measurements for sodium. Here we were able to lower the static field to $5 \mathrm{mV} / \mathrm{cm}$, which depresses the limit by $13 \mathrm{GHz}$. As in Li, we observe excitation to the extremely high-lying states with $n>250$ after the microwave pulse. Scanning the $3 d-n f$ laser frequency while detecting the high-lying states yields a spectrum essentially identical to that shown in Fig. 5.6. High-lying states are again observed for $n \geq 21$ because the maximum microwave field is the same. In the following, the $3 d-n f$ laser was fixed at several different states, and survival and ionization probabilities were taken as described in the section 3.2.2. In Fig. 5.9 we show again the apparent microwave ionization signals obtained with the positive FIP by filled circles, triangles, and squares for the $24 f, 30 f$, and $40 f$ states, respectively. The static field is in all cases $5 \mathrm{mV} / \mathrm{cm}$. In 
addition, we measured a survival probability from the high-lying states with $n>250$ for the same laser frequencies as represented by the open symbols in the figure. As shown, we observe a substantial fraction of the initial population in states with $n>250$ after the microwave pulse, with maximum transfer up to $20 \%$. As none of these low Rydberg states can be ionized by the FIP, a normalization of the population in the high-lying states is done by comparison of the MCP signals to a corresponding signal recorded from one of the higher Rydberg states, for instance, $n=73$, which can be completely ionized by the FIP. As in $\mathrm{Li}$, the population observed in the high-lying states decreases as the microwave field is increased, and the highly excited states appear at the microwave field close to the onset of microwave ionization. To stress again an importance of static fields to microwave ionization experiments, we show Fig. 5.10. Identical to the result of Li experiment, the population in the high-lying states of $\mathrm{Na}$ is quickly ionized by very small static fields. When the laser is tuned to the $30 f$ state, no population can be found in the high-lying states if the static field exceeds $30 \mathrm{mV} / \mathrm{cm}$.

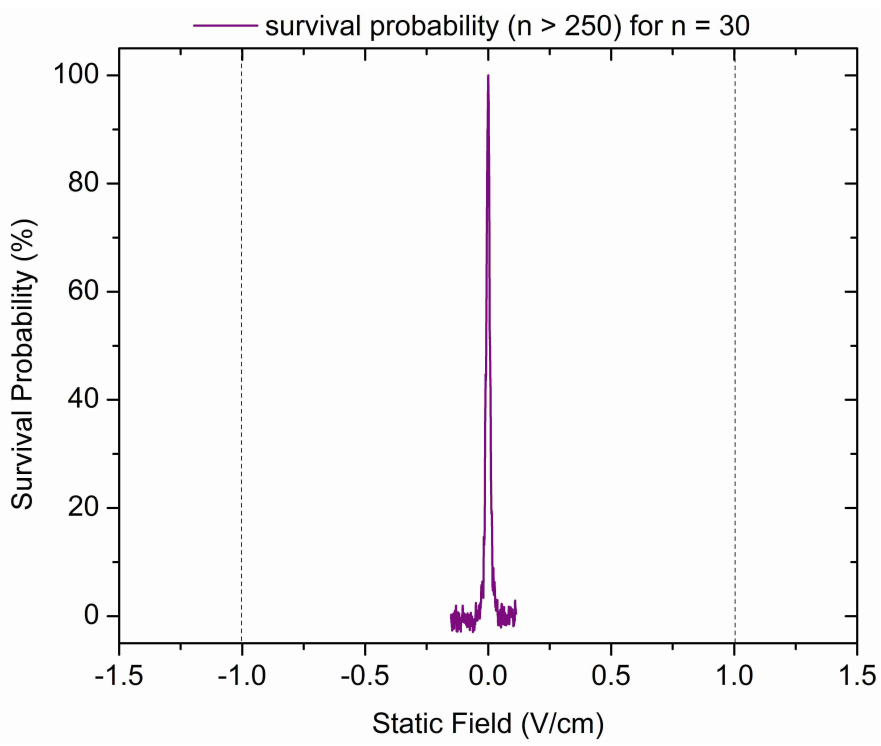

Figure 5.10: The static field dependence of the high-lying state signal of the $30 f$ after exposure to a 120$\mathrm{V} / \mathrm{cm}$ microwave pulse. The high-lying state signal observed by detecting electrons with a negative FIP (solid line). Just as for Li, no bound atoms are detected in the high-lying states when the static field is about $35 \mathrm{mV} / \mathrm{cm}$, a field that ionizes states as low as $\mathrm{n}=360$. Vertical dashed lines at $1 \mathrm{~V} / \mathrm{cm}$ show the static field that was, most likely, present in previous ionization experiments that precluded an observation of the high-lying states.

The results reported here show that the high-lying states with $n>360$ are observed in 
the 16.91-GHz ionization of both $\mathrm{Li}$ and $\mathrm{Na}$ atoms. The most probable reason for their not being observed previously is the requirement of such a low stray field, a field roughly four orders of magnitude weaker than the microwave fields used for ionization. It is not yet clear what sets the upper limit on the static field in which high-lying states can be observed, but it seems to increase with the microwave frequency. In the previous measurements at $38 \mathrm{GHz}$, the static field at which half the high-lying states disappeared was $\approx 35 \mathrm{mV} / \mathrm{cm}$, while in these measurements it is $\approx 17 \mathrm{mV} / \mathrm{cm}$. These static fields depress the ionization limit by 33 $\mathrm{GHz}$ and $22 \mathrm{GHz}$, respectively, so the requirement might be as simple as depressing the limit by the microwave frequency. The observations of the experiments discussed above suggest that the high-lying states are probably produced quite generally in microwave ionization. However, the 80-GHz ionization experiment that we conducted later clarified that the highlying state production is related to the ionization limit and is not a result of the microwave ionization of an initial state.

\subsection{Production of extremely high-lying states in $80-\mathrm{GHz}$ fields}

An observation of the atomic population trapped in the extremely high-lying states after 38 and 17-GHz microwave field pulses for $\mathrm{Li}$ and $\mathrm{Na}$ atoms excited in zero microwave field suggested that this phenomenon is a consequence of the microwave ionization of Rydberg atoms. Our results indicated that the production of the high-lying states is a general phenomenon that occurs for any atom in a Rydberg state that can be at least partially ionized by the microwave field. In other words, a requirement for the observation of the high-lying states is that some fraction of atoms in the initial state is ionizing, and the ejected electron might recombine with the parent ion in the microwave field. To confirm that hypothesis, we conducted an experiment aiming to observe a production of the high-lying states after a strong $80-\mathrm{GHz}$ microwave pulse. An obvious fundamental difference between experiments at 17 and $80 \mathrm{GHz}$ is the size of the microwave photon and the pondermotive shifts that Rydberg electron experiences in the field. At $17 \mathrm{GHz}$, the microwave photon is smaller than a ponderomotive shift $(90 \mathrm{GHz}$ at $100 \mathrm{~V} / \mathrm{cm}$ ), while, for $80 \mathrm{GHz}$ field, it is 
much larger than the shift ( $4 \mathrm{GHz}$ at $100 \mathrm{~V} / \mathrm{cm}$ ). Our goal is to study the high-lying states when the laser is scanned over low Rydberg states of $\mathrm{Na}$ that are later exposed to ionizing 80-GHz microwave field.

\subsubsection{Experimental Approach}

A thermal beam of ground state sodium atoms crosses the microwave electric field antinode at the center of a $79.05-\mathrm{GHz}$ Fabry-Perot microwave cavity. There atoms are excited to a Rydberg state by a sequence of transitions $3 s \rightarrow 3 p \rightarrow 3 d \rightarrow n f$ induced by two 20-ns dye laser pulses and a continuous-wave diode laser. After the laser excitation, atoms are exposed to a microwave pulse, typically $500 \mathrm{~ns}$ long, as shown in Fig. 3.12 (b). About $300 \mathrm{~ns}$ after the end of the pulse, atoms are exposed to a $1-\mu$ s rise time field ionization pulse. Depending on the polarity of the FIP, either electrons or ions are sent upwards to a dual microchannel plate detector. The MCP signal is amplified, captured by a gated integrator or oscilloscope, and recorded in a computer for later analysis. The integration gate or the FIP amplitude can be varied in order to detect only signal from particular range of Rydberg energies.

The Fabry-Perot microwave cavity consists of two brass mirrors $69.7 \mathrm{~mm}$ in diameter with 52 -mm radii of curvature. The on-axis spacing between mirrors is $90.51 \mathrm{~mm}$. The cavity is operated on the $\mathrm{TEM}_{048}$ mode at a frequency of $79.05 \mathrm{GHz}$ with $Q=8600$. The microwave system generates a $79.05-\mathrm{GHz}$ pulse with 0 - to $180 \mathrm{~V} / \mathrm{cm}$ amplitude and a variable width. The experiment is triggered at the $1-\mathrm{kHz}$ repetition rate of the frequencydoubled Nd:YLF laser. The excitation laser beams are sent to the vacuum chamber, through 1-cm holes in the side plates surrounding the cavity, and are focused to less than 1-mm diameter spots where they cross with the atomic beam. Unless stated otherwise, the laser field and microwave field are polarized vertically.

\subsubsection{Experimental Observations}

Our previous experimental endeavors suggested that ionization of Rydberg atoms is a requirement for the observation of the high-lying states trapped after the microwave pulse. 


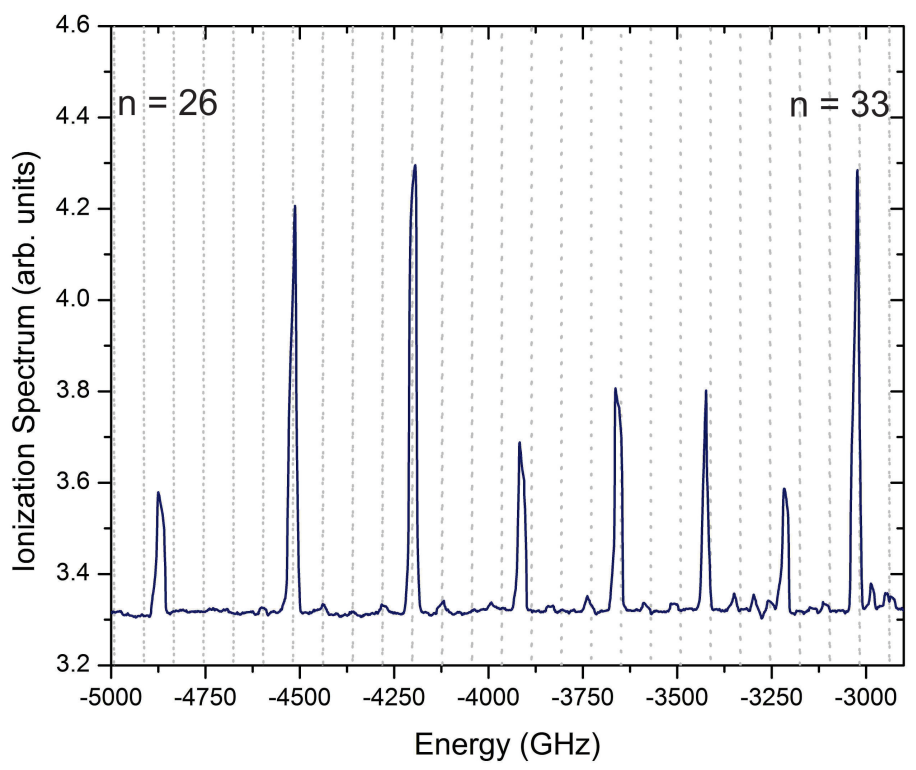

Figure 5.11: Field ionization signal as a function of the laser frequency between $n=26$ and 33 . Ions produced by the microwave field are detected, as the FIP amplitude is not sufficient to ionize these states (only those with $n>50$ ), so a microwave ionization spectrum is detected. After the laser excitation, atoms are exposed to a $170-\mathrm{V} / \mathrm{cm} 500-\mathrm{ns}$ microwave pulse. It is evident that microwave field ionized substantial number of atoms from all excited Rydberg states.

Consequently, we started with measuring an ionization spectrum of lower Rydberg states by detecting ions with the FIP not large enough to ionize Rydberg states of interest. Fig. 5.11 shows the ionization spectrum of Rydberg states between $n=26$ and 33 ionized by $170-\mathrm{V} / \mathrm{cm} 80-\mathrm{GHz}$ microwave pulse. It is evident that the microwave pulse amplitude is large enough to ionize a significant number of atoms from all of these states. More details and results on microwave ionization at $80 \mathrm{GHz}$ can be found in section 3.4.

Fig. 5.11 suggests that all Rydberg states above $n=26$ are substantially ionized by the microwave field, so, if the conclusions of 17 and 38-GHz experiments are correct, the highlying states should be observed when the laser is tuned to any of these states. To explicitly check that, we lowered amplitude of the negative FIP to $0.4 \mathrm{~V} / \mathrm{cm}$ to detect only the highly excited states within $50 \mathrm{GHz}$ of the ionization limit and repeated the measurement of Fig. 5.11. Fig. 5.12 shows the spectrum of the high-lying states that survive the $80-\mathrm{GHz} 170-$ $\mathrm{V} / \mathrm{cm}$ microwave pulse. Surprisingly, we observe a non-zero signal from the high-lying states only when the laser is resonant with some of the Rydberg states but not all. Vertical arrows on the graph indicate peaks that are missing from the spectrum. We did not observe any 
population in the high-lying states when the laser excited $n=29,31$, and 32 , even though these states are ionized by the microwave field as can be seen in Fig. 5.11.

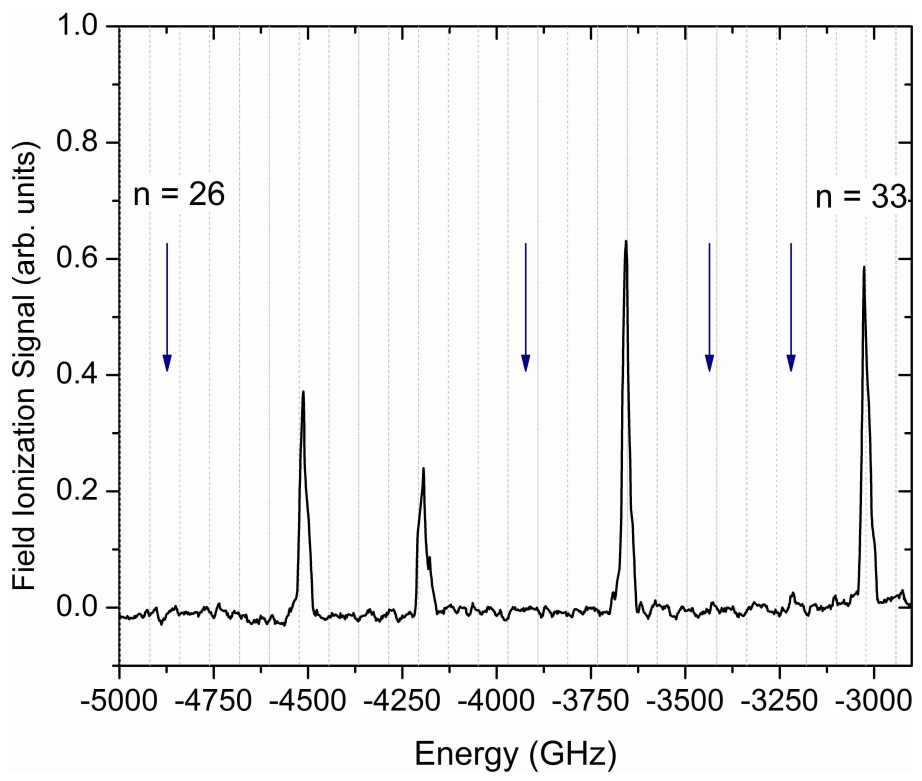

Figure 5.12: Field ionization signal as a function of the laser frequency between $n=26$ and 33. Microwave field amplitude is $170 \mathrm{~V} / \mathrm{cm}$. Electrons are detected, and the FIP amplitude can ionize states within $50 \mathrm{GHz}$ of the IL, so a survival probability from the extremely high-lying states is detected. Vertical arrows indicate energies of zero-field Rydberg states where no peaks are present in the measured spectrum. Vertical dotted lines are spaced by a microwave photon from the IL.

An observation of spectra of the high-lying states obtained by laser excitation in the presence of the microwave field yielded a structure of peaks spaced by a microwave photon from the ionization limit. We wondered if the same requirement has to be fulfilled when atoms are excited in zero-field and counted microwave photons from the ionization limit as shown by the vertical dotted lines in Fig. 5.12. It is evident that we observe a peak in the spectrum only when it is within $10 \mathrm{GHz}$ of the vertical line. In other word, our observations suggest that, for the $80-\mathrm{GHz}$ microwave field, the initial state must be in a multiphoton resonance with the ionization limit in order to trap atoms in the high-lying states. In order to experimentally confirm that notion, we need to either change the energy of the ionization limit by depressing it or change the size of the microwave photon in order to modify multiphoton resonance conditions. The latter can be achieved by varying the spacing between the microwave cavity mirrors and altering the resonant frequency. One of the missing peaks in Fig. 5.12, $n=29$, is about 50 microwave photons from the ionization 

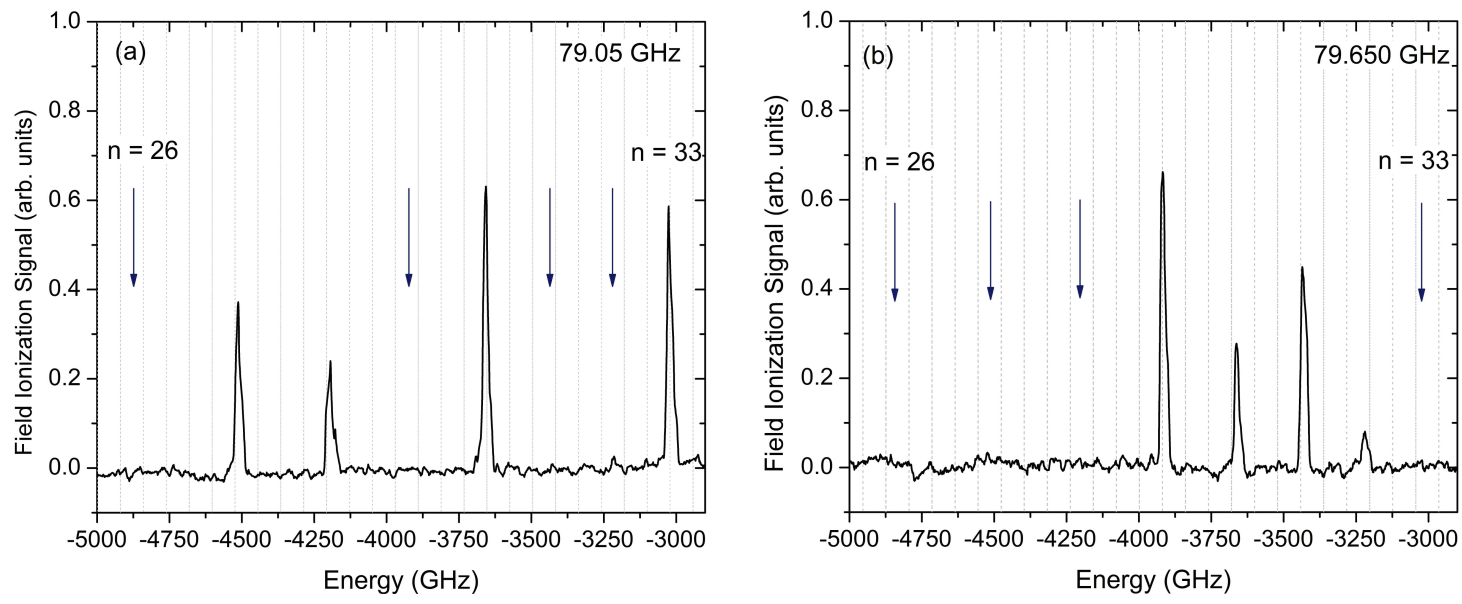

Figure 5.13: Field ionization signal as a function of the laser frequency between $n=26$ and 33 . Microwave field amplitude is $170 \mathrm{~V} / \mathrm{cm}$, and the frequency is $79.05 \mathrm{GHz}$ (a) and $79.65 \mathrm{GHz}$ (b). Electrons are detected, and the FIP amplitude can ionize states within $50 \mathrm{GHz}$ of the IL, so a survival probability from the extremely high-lying states is detected. Vertical arrows indicate energies of zero-field Rydberg states where no peaks are present in the measured spectrum. Vertical dotted lines are spaced by a microwave photon from the IL. Vertical lines in (a) are off by approximately $30 \mathrm{GHz}$ from those in (b). Consequently, different zero-field states are in resonance with the IL, and a different set of peaks is observed.

limit, so, varying the resonant microwave frequency by $0.6 \mathrm{GHz}$ results in $30 \mathrm{GHz}$ shift of the 50th microwave photon position and brings $n=29$ in resonance with the ionization limit. In Fig. 5.13 we show the results of two characteristic measurements taken under identical conditions except a $0.6-\mathrm{GHz}$ difference in microwave frequencies. It is clear that, for the 79.65 microwave field, only $n=29,30,31$, and, partially, $n=32$ Rydberg states are in a multiphoton resonance with the ionization limit, and a non-zero signal from the high-lying states is observed only for these energies. Moreover, the missing peaks of Fig. 5.13 (a) appear in Fig. 5.13 (b), while a signal from the high-lying states at $n=26$ is still not observed as it is not in resonance with the ionization limit in both cases (this state is partially ionized in all cases). However, we additionally modified the microwave frequency to observe a different set of peaks for each frequency. For example, a peak at the energy corresponding to the $n=26$ state is apparent when the microwave field is $79.42 \mathrm{GHz}$.

These new findings indicate that, in contrast to observation at 17 and $38-\mathrm{GHz}$ experiments, the high-lying states are produced only if the laser excites atoms to a low Rydberg state that is resonant with the ionization limit. A similar result we observed in a region within several photons of the ionization limit: comparison of Figs. 3.22 and 3.23 in Section 


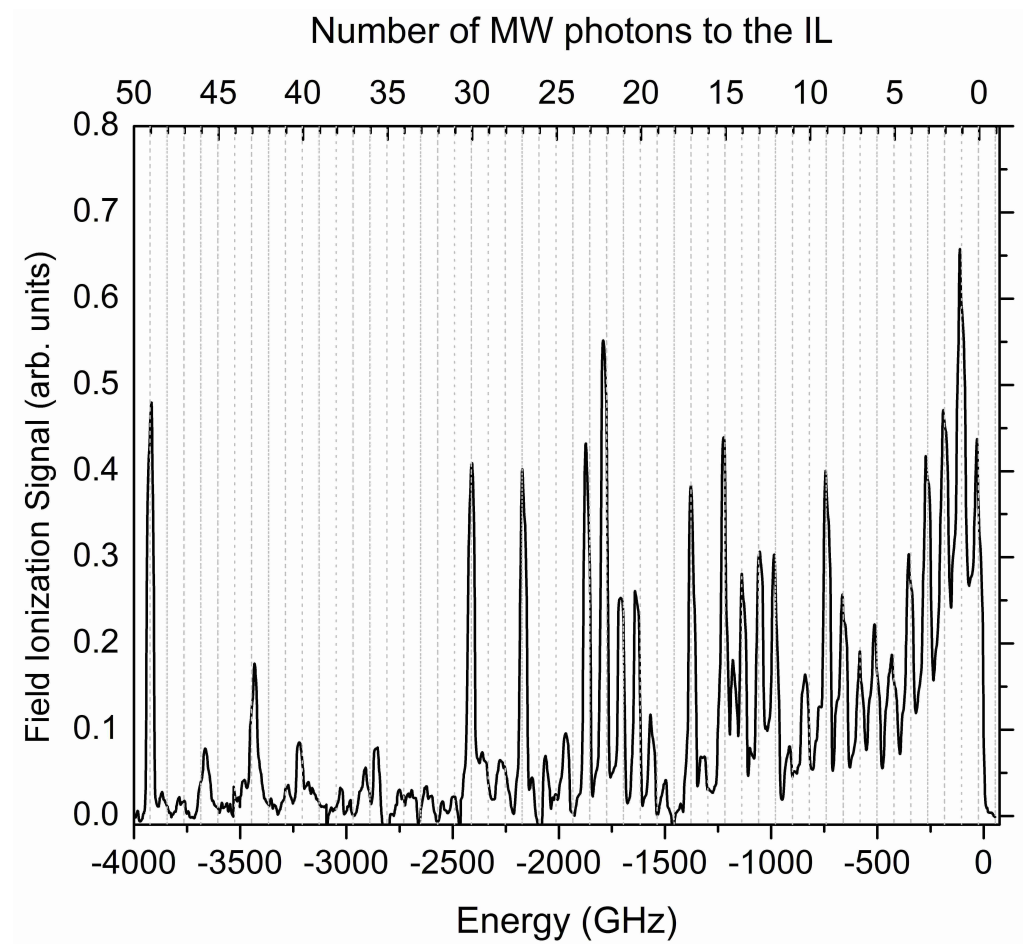

Figure 5.14: Field ionization signal as a function of the laser frequency for $170-\mathrm{V} / \mathrm{cm} 79.05-\mathrm{GHz}$ microwave field. The first peak at $-3910 \mathrm{GHz}$ is at the frequency corresponding to $n=29$. The static field is increased to shift the IL by $25 \mathrm{GHz}$. Electrons are detected, and the FIP amplitude can ionize states within $50 \mathrm{GHz}$ of the IL, so a survival probability from the extremely high-lying states is detected. Vertical dotted lines are spaced by a microwave photon from the IL. Non-zero signal is detected only if the initial state is in resonance with the IL for the whole range of laser tunings.

3.4 showed that atoms are trapped in the high-lying states only when the laser is tuned an integral number of microwave photons from the first peak below the limit. In order to connect these energy regions, we recorded a signal from the high-lying states scanning the laser from low Rydberg states to the ionization limit. The microwave frequency was at $79.05 \mathrm{GHz}$, and we increased the static field to $18 \mathrm{mV} / \mathrm{cm}$ in order to shift the ionization limit by $\sim 25 \mathrm{GHz}$. Such static field decreases the number of observed atoms in the high-lying states by a factor of $\sim 5$. The resulting spectrum is presented in Fig. 5.14. First, by depressing the ionization limit, we brought it into a multiphoton resonance with $n=29$ which resulted in a large peak at this energy as opposed to no signal in Fig. 5.12. Second, as expected, we detect significantly smaller signal from the high-lying states due to the increased static field. Most important, we detect the spectrum that is modulated by the microwave frequency: similar to the measurement taken when the laser excitation is 


\section{Number of MW photons to the IL}

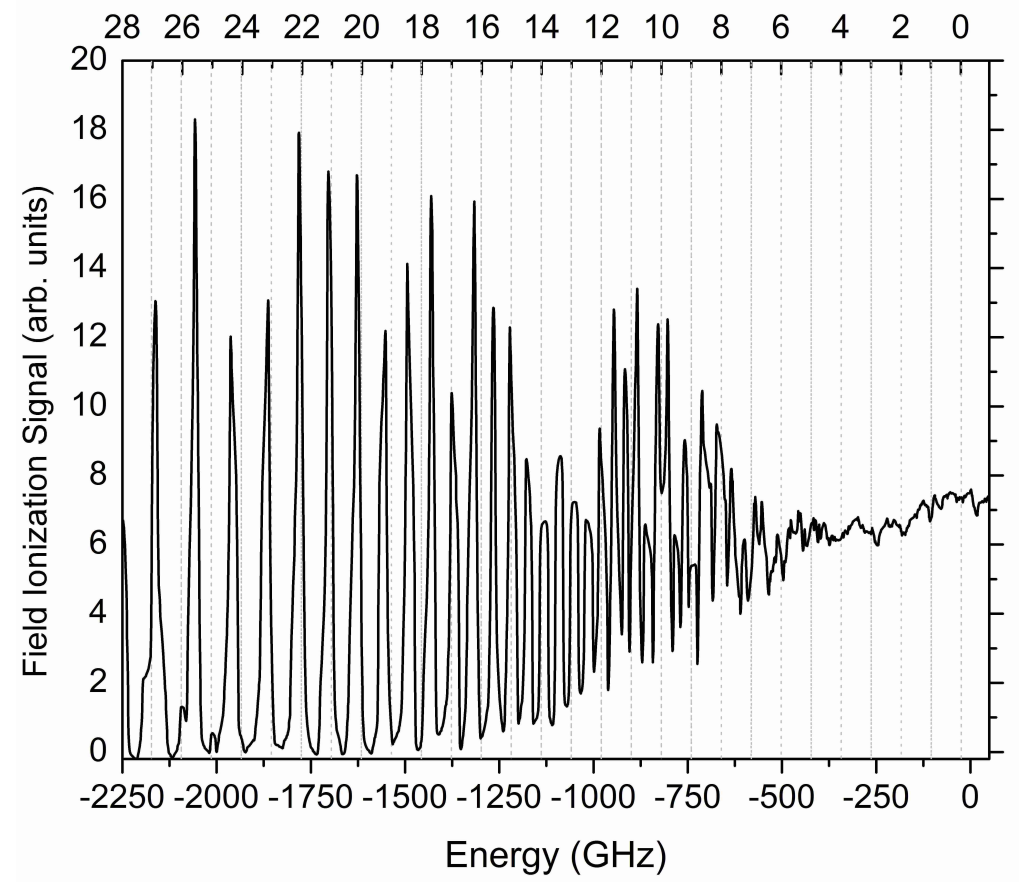

Figure 5.15: Field ionization signal as a function of the laser frequency for $170-\mathrm{V} / \mathrm{cm} 79.05-\mathrm{GHz}$ microwave field. The static field is increased to shift the IL by $25 \mathrm{GHz}$. Ions are detected. FIP ionizes atoms with $n \sim 90$. Vertical dotted lines are spaced by a microwave photon from the IL.

in the presence of the microwave field, we detect non-zero signal from the high-lying states only when the laser is tuned a multiple of microwave photons from the first peak below the ionization limit. There is, though, an obvious difference between these experiments. When the laser pulse is during the microwave field, the high-lying states are populated every time the laser is displaced an integral number of microwave photons from the limit. In case of Fig. 5.14 however, the laser can only excite population to a zero-field Rydberg state, so it is possible to find the population in the high-lying states only when the initial Rydberg state is in a multiphoton resonance with the limit at $80 \mathrm{GHz}$. Thus, the spectrum of Fig. 5.14 does not exhibit the same regularity as a spectrum of Fig. 4.11 where a regular structure of peaks is observed. It is also useful to compare the spectrum of Fig. 5.14 to the one shown in Fig. 5.15, which was taken under identical conditions but detecting ions with a larger FIP capable of ionizing states with $n>90$. It is clear that at this microwave field amplitude all atoms are ionized for the range of binding energies shown, and peaks are recorded for 
all Rydberg state frequencies as opposed to the spectrum of the high-lying states when the initial states has to be displaced from the limit by an integral number of microwave photons.

The results of the experiments at 38 and $17 \mathrm{GHz}$ showed that the production of the high-lying states always accompanies the microwave ionization. A likely reason for the disagreement in experimental observations is the difference in the ponderomotive shifts that the Rydberg electron experiences at different microwave frequencies. A $100-\mathrm{V} / \mathrm{cm}$ field at 17, 38, and $80 \mathrm{GHz}$ shifts the limit by 93,19 , and $4 \mathrm{GHz}$ respectively. So, at $17 \mathrm{GHz}$ the pondermotive shift is almost five times bigger than the microwave photon, while at $80 \mathrm{GHz}$ it is about 20 times smaller. Our observations point out that low Rydberg states do not experience substantial shift in energy, as was discussed in Section 3.4.2, so we can conclude that only the limit shifts ponderomotively or due to the static field. Consequently, we can assume that, on the rising edge of the microwave pulse, an initial state is brought into resonance with the ionization limit about five times at $17 \mathrm{GHz}$, but not at $80 \mathrm{GHz}$, where different states should shift into resonance at different intensities. Thus, at $17 \mathrm{GHz}$ the high-lying states are always produced, while at $80 \mathrm{GHz}$ no high-lying states are produced if the initial state can not be shifted into resonance with the limit. Further experimental work is required to confirm that notion.

\subsection{Conclusions}

An unexpected and surprising discovery that we made conducting the microwave ionization experiments is the production of the high-lying states following the microwave pulse. When atoms of lithium excited to a Rydberg state were exposed to a strong $38-\mathrm{GHz}$ microwave pulse, about $5-10 \%$ of atoms were found bound in the form of extremely high-lying states with $n>250$. Atoms left in these high-lying states showed a peculiar resistance to an increased microwave field strength and number of cycles in the pulse, but were ionized by small static fields. That novel phenomenon turned out to occur not only during ionization of $\mathrm{Li}$ atoms by $38-\mathrm{GHz}$ fields, but was also observed for $17-\mathrm{GHz}$ ionizing fields. We detected no difference in results of the experiments on $\mathrm{Li}$ or $\mathrm{Na}$ atoms. The production of the high- 
lying states seemed to be a general phenomenon that results from the microwave ionization of Rydberg atoms that was not detected in previous microwave ionization experiments due to relatively large static fields and ion detection techniques.

It is useful to compare our results to those of Zhao et al., who examined both experimentally and theoretically the effect of trains of short unipolar pulses with an added bias field. They studied cases in which the scaled frequency $\Omega$ was both less than and greater than one. In the experiments, with trains of up to 40 pulses, they found atoms were most likely to be found in high-lying states subsequent to the pulse train if the bias field resulted in a time average field of zero. This finding is similar to our observation that the high-lying states are only observed when the stray fields are less than $30 \mathrm{mV} / \mathrm{cm}$. The classical calculations of Zhao et al., which included trains of up to 200 pulses, also show that the high-lying states survive longest when the bias field results in a vanishing time average field. They attribute the stability to the fact that the time average field is zero, leading to a vanishing average force. With a train of 200 pulses the calculations show a $78 \%$ survival probability if the atoms are initially in a state for which $\Omega=3.3$ but only a $10 \%$ survival if the atoms are in a relatively low lying state, one for which $\Omega=0.3$. Assuming an exponential decay of the high-lying states, extrapolation of these results to our microwave pulses, which contain 7900 cycles, yields a survival probability of $5 \times 10^{-5}$ for $\Omega=3.3$, and $10^{-39}$ for $\Omega=0.3$. These survival probabilities are much lower than our observed survival probabilities of $5 \%$ in both cases. It is important to mention though that the observation of the high-lying states in the microwave field is different from the experiment of Zhao et al. who considers the case when the applied train of pulses can interact with the Rydberg electron during the whole orbit. They discuss that the static field cancels out an effect of the pulse train resulting in a zero force averaged over one orbit. However, periodic and dc fields interact with atoms differently: the static field can only ionize an atom if the resulting energy of the Rydberg electron is over the modified Coulomb-Stark barrier, and its position relative to the core is irrelevant. In the microwave field, on the other hand, when $\Omega>1$, if the electron is far from the core, it oscillates as a free charge and can exchange energy only when it is close to the core. 
We observed that atoms are left in the high-lying states after the microwave pulse for any initial state that can be at least partially ionized by 17 and $38-\mathrm{GHz}$ fields. That suggested that the high-lying states are produced when ionization occurs. We tried to explain it as a rescattering process similar to a "frustrated tunneling ionization" of Nubbemeyer et al. [36]. They found population left bound in excited states centered around $n=8$ after ionizing ground state atoms of He by ultra-strong femtosecond laser pulse. An essential idea is that an ejected electron stays in the vicinity of the Coulomb potential and has a chance to recombine with the parent ion by losing energy to the periodic laser or microwave field. It is suggested by the Simpleman's model [39, 40]. It predicts that electrons ionized at the peak of the microwave field have zero drift velocity, only an oscillation in the field, so the electron remains near the ion core, oscillating in the field. The Simpleman's model must be modified to take into account the Coulomb potential [38], but the basic idea is unchanged; microwave ionization at some phase of the microwave field produces electrons with zero time average velocity.

The hypothesis that the high-lying states results from ionization and subsequent recombination of atoms, however, was not confirmed by the $80 \mathrm{GHz}$ experiment. Our results clearly showed that ionization of atoms from the initially excited state does not guarantee the production of the high-lying states. We observed no atoms in the high-lying states when the laser was tuned to a Rydberg state that was not in resonance with the ionization limit. The similar effect must happen in microwave fields below $80 \mathrm{GHz}$, but could not be observed experimentally, as the ionization limit can be shifted in resonance multiple times during the rising edge of the microwave pulse, and the high-lying states were always detected for any initial state.

Thus, it appears that we observe a similar phenomena when the laser excitation atoms occurs in the presence of the microwave field and when already excited atoms are later exposed to the microwave field. In both cases we observe atoms trapped in the highlying states only if the initial state is displaced by integral multiples of the microwave frequency from the ionization limit (the first peak below it). Consequently, when the laser is tuned below the ionization limit, atoms are driven into the high-lying states not due to a 
recombination process, but rather a multiphoton excitation to the limit. In other words, that phenomenon occurs not because of the microwave ionization but along with it. Essentially the same result was observed in calculations to elucidate the source of superponderomotive electrons [58]. Superponderomotive electrons are observed in the ATI spectrum and have energies in excess of the Simpleman's model predictions. The yield of such electrons is resonantly enhanced when the ionization potential of the ponderomotively shifted initial state equals $(N+0.5) h \nu$. That suggests that the strong enhancement is due to atoms in states half a photon below the threshold. In other words, superponderomotive electrons are observed at the intensities which bring states just below the limit into multiphoton resonance with the ground state. In the usual case of ionization by a pulse of microwaves or laser light, the Stark shift due to the changing intensity during the pulse leads to resonant excitation of the high-lying states, as shown by the calculations of Muller [58]. Thus, not only ionization but also excitation can occur both in tunneling and multiphoton regimes. It is unclear though why atoms have to be excited to the highly-excited states only below the limit. As our experimental technique does not allow us to detect low energy electrons with energies just above the threshold, we can not conclude that multiphoton excitation happens to the states below the limit only. For example, recent discoveries of low energy structure in the ATI spectrum $[31,32,33]$ might be due to the same phenomenon: multiphoton excitation of atoms to the ionization threshold with some energy spread below and above the limit by the external periodic, laser or microwave, field. 


\section{Chapter 6}

\section{Conclusions}

This chapter summarizes the conclusions of the experiments discussed in this dissertation. The ionization experiment conducted at $16.91 \mathrm{GHz}$ revealed that, at low $n, \mathrm{Na}|m|=1$ atoms require a microwave field of $E=1 / 9 n^{4}$ for ionization in zero static field, but it is reduced to $E=1 / 3 n^{5}$ if a static field of $\sim 1 \mathrm{~V} / \mathrm{cm}$ is present. We also reported an investigation of microwave ionization of $\mathrm{Li}$ atoms at $38 \mathrm{GHz}$, using the same apparatus, from low $n$, where $\Omega \approx 1 / 3$, to high enough $n$ that single photon ionization by the microwave field is possible. The fields required to effect $10 \%$ and $50 \%$ ionization decrease slowly with $n$ in the $\Omega<1$ regime and are almost constant in the $\Omega>1$ regime, except very close to the ionization limit, where pronounced structure is observed in the ionization fields, due to the atoms' being left in highly excited states. These observations are consistent with previous measurements which covered pieces of the range of $n$ explored here.

The $\mathrm{Na}$ ionization experiment at $80 \mathrm{GHz}$ showed that, in the $\Omega<1$ regime, ionization occurs at fields higher than $E=1 / 3 n^{5}$ due to the fact that the coupling between extreme Stark states of adjacent manifolds is much smaller than the field frequency, and no $n \rightarrow n+1$ transition occurs, which is the rate-limiting step when ionization occurs at $E=1 / 3 n^{5}$. In this region, the ionization fields are modified by occasional resonances, when the spacing between zero-field states is a multiple of the microwave photon, and can be lowered by adding $4.5-\mathrm{V} / \mathrm{cm}$ static field that enhances the resonances for some states. Moreover, the observed ionization fields are strongly dependent upon the width of the $80-\mathrm{GHz}$ microwave pulse, a result different from the conclusions of the experiments conducted below $20 \mathrm{GHz}$. 
In the $\Omega>1$ region, ionization fields are on average $n$ independent, which is consistent with 17 and 38-GHz experiments and the theoretical predictions of Jensen et al., up to $\sim 6$ photons below the limit. At this point photoionization rates become significant. The experimentally measured single photon ionization rates are in a good agreement with the prediction of perturbation theory.

Most interesting, in the ionization of $\mathrm{Li}$ and $\mathrm{Na}$ atoms by the $16.91 \mathrm{GHz}$ microwave field, roughly $15 \%$ of the atoms are not actually ionized but trapped in highly excited states within one or, at most, two microwave photons of the ionization limit. The highly excited atoms are only detected in static fields less than $35 \mathrm{mV} / \mathrm{cm}$, a field which roughly corresponds to the $1 / 16 n^{4}$ field ionization of an atom bound by twice the microwave frequency. Our results also point out that static fields on the order of $1 \mathrm{~V} / \mathrm{cm}$ were present in earlier microwave ionization experiments conducted in waveguides below $20 \mathrm{GHz}$ that must have precluded an observation of the high-lying states.

A similar phenomenon is observed at $38 \mathrm{GHz}$ ionization of Li Rydberg atoms. When atoms are exposed to intense microwave pulses, in spite of the fact that the pulse is more than 8000 cycles long, $\sim 10 \%$ of the atoms are found in extremely high Rydberg states subsequent to the pulse, even if it is far more intense than required for static field ionization of such states. Surprisingly, atoms in such high-lying states are very stable in the microwave field and can survive Rydberg electron visiting the core more than once. Our work shows quite clearly that the production of high-lying states in strong microwave fields occurs quite generally; it is not peculiar to Li or high microwave frequencies, those in excess of $30 \mathrm{GHz}$.

The results of the experiments conducted at 17 and $38 \mathrm{GHz}$ suggested that the highlying states should be observed for any Rydberg state that an be at least partially ionized by the microwave field. However, the $80-\mathrm{GHz}$ experiment clarified that the production of the high-lying states is related to the ionization limit. Due to the fact that the ponderomotive shift at $80 \mathrm{GHz}$ is much smaller than the size of the microwave photon, in contrast to the 17-GHz case, the high-lying states are found not for any initial state, even if that state is ionized substantially. The high-lying states are observed at $80 \mathrm{GHz}$ only when the initial state in in the multiphoton resonance with the shifted ionization limit. Similarly, when 
atoms are excited in the presence of the microwave field, the extremely high-lying states are also observed, but in the form of a regular structure of peaks, spaced by a microwave photon, that extends far below the limit $(\Omega \approx 0.2)$ and more than several microwave photons above it. This structure is also tied to the ionization limit, as all peaks experience the same energy shift due to the dc and microwave fields as does the ionization limit. The results of the 80-GHz experiment also suggest that excitation to the high-lying states does not happen via real $n \rightarrow n+1$ transitions, but, possibly, through the Floquet states emerging from the ionization limit.

Overall, the most astonishing result of the conducted experiments is an observation of a substantial fraction of atoms left in the extremely high-lying states after a strong microwave pulse. This phenomenon is observable for any microwave frequency we tried and different atomic species, Li and Na. The production of the high-lying states appears to be a process that occurs along with microwave ionization of Rydberg atoms but is not an after-effect of it. 


\section{Bibliography}

[1] Thomas F. Gallagher. Rydberg Atoms. Cambridge University Press, 1994. Cambridge Books Online.

[2] Hans A Bethe and Edwin E Salpeter. Quantum mechanics of one- and two-electron atoms. Dover, New York, NY, 2008.

[3] L. V. Keldysh. Ionization in the field of a strong electromagnetic wave. Soviet Physics JETP, 20:1307-1314, May 1965.

[4] Benjamin J. Sussman. Five ways to the nonresonant dynamic stark effect. American Journal of Physics, 79(5):477-484, 2011.

[5] N. B. Delone and V. P. Krainov. Tunneling and barrier-suppression ionization of atoms and ions in a laser radiation field. Physics-Uspekhi, 41(5):469-485, 1998.

[6] P. Agostini, F. Fabre, G. Mainfray, G. Petite, and N. K. Rahman. Free-free transitions following six-photon ionization of xenon atoms. Phys. Rev. Lett., 42:1127-1130, Apr 1979 .

[7] R. R. Freeman, P. H. Bucksbaum, H. Milchberg, S. Darack, D. Schumacher, and M. E. Geusic. Above-threshold ionization with subpicosecond laser pulses. Phys. Rev. Lett., 59:1092-1095, Sep 1987.

[8] M. P. de Boer and H. G. Muller. Observation of large populations in excited states after short-pulse multiphoton ionization. Phys. Rev. Lett., 68:2747-2750, May 1992.

[9] A. l'Huillier, L. A. Lompre, G. Mainfray, and C. Manus. Multiply charged ions induced by multiphoton absorption in rare gases at $0.53 \mu \mathrm{m}$. Phys. Rev. A, 27:2503-2512, May 1983.

[10] P. A. M. Dirac. The quantum theory of the emission and absorption of radiation. Proc. R. Soc., London, Sec. A, 114(767):243-265, 1927.

[11] H. Maeda, J. H. Gurian, D. V. L. Norum, and T. F. Gallagher. Coherent population transfer in an atom by multiphoton adiabatic rapid passage. Phys. Rev. Lett., 96:073002, Feb 2006.

[12] H. Maeda, J. H. Gurian, and T. F. Gallagher. Nondispersing bohr wave packets. Phys. Rev. Lett., 102:103001, Mar 2009. 
[13] P. Pillet, H. B. van Linden van den Heuvell, W. W. Smith, R. Kachru, N. H. Tran, and T. F. Gallagher. Microwave ionization of na rydberg atoms. Phys. Rev. A, 30:280-294, Jul 1984.

[14] P. Pillet, W. W. Smith, R. Kachru, N. H. Tran, and T. F. Gallagher. Microwave ionization of na rydberg levels. Phys. Rev. Lett., 50:1042-1045, Apr 1983.

[15] J. E. Bayfield and P. M. Koch. Multiphoton ionization of highly excited hydrogen atoms. Phys. Rev. Lett., 33:258-261, Jul 1974.

[16] C. R. Mahon, J. L. Dexter, P. Pillet, and T. F. Gallagher. Ionization of sodium and lithium rydberg atoms by 10-mhz to 15-ghz electric fields. Phys. Rev. A, 44:1859-1873, Aug 1991.

[17] U. Eichmann, J.L. Dexter, E.Y. Xu, and T.F. Gallagher. Microwave ionization and excitation of ba rydberg atoms. Zeitschrift fr Physik D Atoms, Molecules and Clusters, 11(3):187-197, 1989.

[18] P. Pillet, C. H. Mahon, and T. F. Gallagher. Enhancement of microwave ionization by quasicontinuum production. Phys. Rev. Lett., 60:21-24, Jan 1988.

[19] H. B. van Linden van den Heuvell, R. Kachru, N. H. Tran, and T. F. Gallagher. Excitation spectrum of na rydberg states in a strong microwave field: A connection between two points of view. Phys. Rev. Lett., 53:1901-1904, Nov 1984.

[20] P. Pillet, R. Kachru, N. H. Tran, W. W. Smith, and T. F. Gallagher. Radiative collisions in a strong-field regime. Phys. Rev. Lett., 50:1763-1766, May 1983.

[21] James E. Bayfield, Larry D. Gardner, and Peter M. Koch. Observation of resonances in the microwave-stimulated multiphoton excitation and ionization of highly excited hydrogen atoms. Phys. Rev. Lett., 39:76-79, Jul 1977.

[22] Andreas Krug and Andreas Buchleitner. Chaotic ionization of nonhydrogenic alkali rydberg states. Phys. Rev. Lett., 86:3538-3541, Apr 2001.

[23] J. H. Gurian, K. R. Overstreet, H. Maeda, and T. F. Gallagher. Connecting field ionization to photoionization via 17- and 36-ghz microwave fields. Phys. Rev. A, 82:043415, Oct 2010 .

[24] H. Maeda and T. F. Gallagher. Quantum suppression of microwave ionization of rydberg atoms at high scaled frequency. Phys. Rev. Lett., 93:193002, Nov 2004.

[25] R. V. Jensen, S. M. Susskind, and M. M. Sanders. Microwave ionization of highly excited hydrogen atoms: A test of the correspondence principle. Phys. Rev. Lett., 62:1476-1479, Mar 1989.

[26] N B Delone, S P Goreslavsky, and V P Krainov. Dipole matrix elements in the quasiclassical approximation. Journal of Physics B: Atomic, Molecular and Optical Physics, 27(19):4403, 1994.

[27] R. R. Jones, D. W. Schumacher, and P. H. Bucksbaum. Population trapping in kr and xe in intense laser fields. Phys. Rev. A, 47:R49-R52, Jan 1993. 
[28] E. Wells, I. Ben-Itzhak, and R. R. Jones. Ionization of atoms by the spatial gradient of the pondermotive potential in a focused laser beam. Phys. Rev. Lett., 93:023001, Jul 2004.

[29] R. R. Jones and P. H. Bucksbaum. Ionization suppression of stark states in intense laser fields. Phys. Rev. Lett., 67:3215-3218, Dec 1991.

[30] H. Stapelfeldt, D. G. Papaioannou, L. D. Noordam, and T. F. Gallagher. Inner-electron multiphoton ionization of barium rydberg states with picosecond pulses. Phys. Rev. Lett., 67:3223-3226, Dec 1991.

[31] P. Colosimo G. G. Paulus H. G. Muller P. Agostini L. F. DiMauro C. I. Blaga, F. Catoire. Strong-field photoionization revisited. Nature Physics, 5:335 - 338, 2008.

[32] W. Quan, Z. Lin, M. Wu, H. Kang, H. Liu, X. Liu, J. Chen, J. Liu, X. T. He, S. G. Chen, H. Xiong, L. Guo, H. Xu, Y. Fu, Y. Cheng, and Z. Z. Xu. Classical aspects in above-threshold ionization with a midinfrared strong laser field. Phys. Rev. Lett., 103:093001, Aug 2009.

[33] L. Guo, S. S. Han, X. Liu, Y. Cheng, Z. Z. Xu, J. Fan, J. Chen, S. G. Chen, W. Becker, C. I. Blaga, A. D. DiChiara, E. Sistrunk, P. Agostini, and L. F. DiMauro. Scaling of the low-energy structure in above-threshold ionization in the tunneling regime: Theory and experiment. Phys. Rev. Lett., 110:013001, Jan 2013.

[34] A. McPherson, G. Gibson, H. Jara, U. Johann, T. S. Luk, I. A. McIntyre, K. Boyer, and C. K. Rhodes. Studies of multiphoton production of vacuum-ultraviolet radiation in the rare gases. J. Opt. Soc. Am. B, 4(4):595-601, Apr 1987.

[35] D. N. Fittinghoff, P. R. Bolton, B. Chang, and K. C. Kulander. Observation of nonsequential double ionization of helium with optical tunneling. Phys. Rev. Lett., 69:26422645, Nov 1992.

[36] T. Nubbemeyer, K. Gorling, A. Saenz, U. Eichmann, and W. Sandner. Strong-field tunneling without ionization. Phys. Rev. Lett., 101:233001, Dec 2008.

[37] U. Eichmann, A. Saenz, S. Eilzer, T. Nubbemeyer, and W. Sandner. Observing rydberg atoms to survive intense laser fields. Phys. Rev. Lett., 110:203002, May 2013.

[38] E. S. Shuman, R. R. Jones, and T. F. Gallagher. Multiphoton assisted recombination. Phys. Rev. Lett., 101:263001, Dec 2008.

[39] H.B. van Linden van den Heuvell and H.G. Muller. Multiphoton processes. Cambridge University Press, Cambridge, 1988.

[40] T. F. Gallagher. Above-threshold ionization in low-frequency limit. Phys. Rev. Lett., 61:2304-2307, Nov 1988.

[41] W Zhao, J C Lancaster, F B Dunning, C O Reinhold, and J Burgdrfer. The periodically kicked atom: effect of the average dc field. Journal of Physics B: Atomic, Molecular and Optical Physics, 38(2):S191, 2005. 
[42] T. J. Bensky, M. B. Campbell, and R. R. Jones. Half-cycle pulse assisted electron-ion recombination. Phys. Rev. Lett., 81:3112-3115, Oct 1998.

[43] C. Wesdorp, F. Robicheaux, and L. D. Noordam. Field-induced electron-ion recombination: A novel route towards neutral (anti-)matter. Phys. Rev. Lett., 84:3799-3802, Apr 2000.

[44] Michael W. Noel, W. M. Griffith, and T. F. Gallagher. Population trapping in extremely highly excited states in microwave ionization. Phys. Rev. Lett., 83:1747-1750, Aug 1999.

[45] John H Moore, Michael A Coplan, and Christopher C Davis. Building scientific apparatus: a practical guide to design and construction. Addison-Wesley, London, 1983.

[46] Michael G. Littman and Harold J. Metcalf. Spectrally narrow pulsed dye laser without beam expander. Appl. Opt., 17(14):2224-2227, Jul 1978.

[47] Joshua H. Gurian. Multiphoton Microwave Ionization of Rydberg Atoms. PhD thesis, University of Virginia, 2010.

[48] Michael G. Littman. Single-mode operation of grazing-incidence pulsed dye laser. Opt. Lett., 3(4):138-140, Oct 1978.

[49] John R. Whinnery Simn Ramo. Fields and waves in modern radio. J. Wiley \& Sons, Inc., New York, 1953.

[50] C. H. Cheng, C. Y. Lee, and T. F. Gallagher. Ionization of li rydberg atoms by 8.5- and 18-ghz circularly polarized microwave fields. Phys. Rev. A, 54:3303-3309, Oct 1996.

[51] K. A. H. van Leeuwen, G. v. Oppen, S. Renwick, J. B. Bowlin, P. M. Koch, R. V. Jensen, O. Rath, D. Richards, and J. G. Leopold. Microwave ionization of hydrogen atoms: Experiment versus classical dynamics. Phys. Rev. Lett., 55:2231-2234, Nov 1985.

[52] Alexej Schelle, Dominique Delande, and Andreas Buchleitner. Microwave-driven atoms: From anderson localization to einstein's photoeffect. Phys. Rev. Lett., 102:183001, May 2009 .

[53] P.M. Koch and K.A.H. van Leeuwen. The importance of resonances in microwave ionization of excited hydrogen atoms. Physics Reports, 255(56):289 - 403, 1995.

[54] G. Casati, I. Guarneri, and D. L. Shepelyansky. Exponential photonic localization for the hydrogen atom in a monochromatic field. Phys. Rev. A, 36:3501-3504, Oct 1987.

[55] R. C. Stoneman, D. S. Thomson, and T. F. Gallagher. Microwave multiphoton transitions between rydberg states of potassium. Phys. Rev. A, 37:1527-1540, Mar 1988.

[56] Michael W. Noel, W. M. Griffith, and T. F. Gallagher. Classical subharmonic resonances in microwave ionization of lithium rydberg atoms. Phys. Rev. A, 62:063401, Oct 2000 . 
[57] A. Arakelyan and T. F. Gallagher. Metastable states in microwave ionization. Phys. Rev. A, 87:023410, Feb 2013.

[58] H. G. Muller. Tunneling excitation to resonant states in helium as main source of superponderomotive photoelectrons in the tunneling regime. Phys. Rev. Lett., 83:31583161, Oct 1999.

[59] K. R. Overstreet, R. R. Jones, and T. F. Gallagher. Phase-dependent electron-ion recombination in a microwave field. Phys. Rev. Lett., 106:033002, Jan 2011.

[60] A. Giusti-Suzor and P. Zoller. Rydberg electrons in laser fields: A finite-rangeinteraction problem. Phys. Rev. A, 36:5178-5188, Dec 1987.

[61] W. E. Cooke and C. L. Cromer. Multichannel quantum-defect theory and an equivalent $N$-level system. Phys. Rev. A, 32:2725-2738, Nov 1985.

[62] A. Arakelyan, T. Topcu, F. Robicheaux, and T. F. Gallagher. Spectrum of quasistable states in a strong microwave field. Phys. Rev. A, 90:013413, Jul 2014.

[63] Walter C. Henneberger. Perturbation method for atoms in intense light beams. Phys. Rev. Lett., 21:838-841, Sep 1968.

[64] M. Pont, N. R. Walet, M. Gavrila, and C. W. McCurdy. Dichotomy of the hydrogen atom in superintense, high-frequency laser fields. Phys. Rev. Lett., 61:939-942, Aug 1988 .

[65] P. B. Corkum, N. H. Burnett, and F. Brunel. Above-threshold ionization in the longwavelength limit. Phys. Rev. Lett., 62:1259-1262, Mar 1989.

[66] B. E. Sauer, M. R. W. Bellermann, and P. M. Koch. Classical scaling of nonclassical stability in microwave ionization of excited 3d h atoms. Phys. Rev. Lett., 68:1633-1636, Mar 1992.

[67] M. P. Robinson, B. Laburthe Tolra, Michael W. Noel, T. F. Gallagher, and P. Pillet. Spontaneous evolution of rydberg atoms into an ultracold plasma. Phys. Rev. Lett., 85:4466-4469, Nov 2000.

[68] J. P. Morrison, C. J. Rennick, J. S. Keller, and E. R. Grant. Evolution from a molecular rydberg gas to an ultracold plasma in a seeded supersonic expansion of no. Phys. Rev. Lett., 101:205005, Nov 2008. 